Distribución del tamaño de partículas

\title{
y microagregados en suelos de la Pradera Pampeana: \\ estudio mediante la geometría fractal
}

\begin{abstract}
Tesis presentada para obtener el título de Doctor de la Facultad Ciencias Agrarias y Forestales, Universidad Nacional de La Plata
\end{abstract}

\section{Pablo Raúl Gelati}

Ingeniero Forestal - FCA y F- UNLP

Director

Dr. Roberto R. Filgueira

FCAyF- UNLP. CONICET
Codirector

Lic. Guillermo O. Sarli

FCAyF - UNLP

Julio de 2020 
Dedicatoria

A Emilia, Juan Martín, Esteban, Natalia y Claudio.

A Ines, Mario y mis hermanos.

A Ch.N.N. 
Agradecimientos:

A, Emilia, Juan Martín, Esteban, Natalia y Claudio, por el apoyo incondicional

A los directores Roberto y Guillermo, por acompañarme y orientarme en este camino

A los señores del jurado por los aportes y predisposición que mejoraron el manuscrito definitivo

A Gabriel, por facilitarme herramientas para investigar

A Ana Arambarri, por su colaboración y predisposición para obtener las fotos microscópicas

A Laura Boff y Marcelo Manassero, por el asesoramiento en las curvas y clasificaciones sedimentológicas

A Daniel Poire, Cecilia Genazzini y Germán Kurten, por las determinaciones de mineralogía

A María Elena Cannofoglia por las determinaciones y colaboración

A Mario da Silva, por su predisposición y apoyo

A Rodrigo Altamirano por el asesoramiento estadístico

A Martín Brouver, Agustín Oderiz, Luis Mateos, Fernando Girón y Agustín Bestilleiro, por facilitarme muestras de suelos

A los grupos de trabajo de Edafología y Física Aplicada 
Declaro que el material incluido en la presente tesis es original, producto de mi propia elaboración, (se han empleado conocimientos previos, los cuales se indican explícitamente) y no es parte o total de otra tesis.

Ing. Ftal. Pablo Raúl Gelati 
Resumen

La granulometría es la característica física más estable del suelo, su conocimiento es fundamental, ya que está relacionada con la fertilidad, el manejo y la clasificación taxonómica. Por otro lado, la granulometría es el principal ingrediente de las ecuaciones de transferencia edafológica. Puede cuantificarse de manera completa mediante el análisis de la distribución de tamaño de partículas (DTP), o en forma resumida a partir de la determinación de textura, donde solamente se estiman los valores totales de las fracciones arena, limo y arcilla. Actualmente, para los relevamientos cartográficos se incorporan valores intermedios, dentro de la fracción limo (limos $2-20 \mu \mathrm{m})$ y arenas muy finas $(50-75 \mu \mathrm{m})$.

El modelo fractal ha sido empleado como marco teórico para describir la DTP de los suelos. Un objeto fractal está compuesto de una cantidad de estructuras similares superpuestas, visibles a cualquier nivel de detalle. Esta propiedad se conoce como autosimilitud. El material en un objeto fractal se distribuye en forma heterogénea, pero no al azar, de forma que un patrón de generación se ve idéntico al menos en el sentido estadístico e independientemente de la amplificación con que se esté observando. En un fractal matemático los detalles se ven idénticos en cualquier nivel de amplificación, mientras que en un fractal natural es esperable que el nivel de repetición de la propiedad particular sea limitado a varios órdenes de magnitud.

Se plantearon como hipótesis: La distribución de partículas y microagregados de suelos de clima templado de la pradera pampeana responden a una distribución fractal. Se puede obtener información sobre el estado de la microestructuración de un suelo a través del estudio de la distribución de partículas, microagregados y el análisis fractal de fragmentación. Los objetivos fueron investigar el comportamiento fractal de la distribución de tamaños de partículas (DTP) y de la distribución de tamaño de microagregados (DTM) del suelo y asociar la distribución de tamaño de partículas con el material original y los procesos de transporte y sedimentación.

Se estudió la DTP y la DTM en muestras de suelos de la provincia de Buenos Aires en un transecto de $500 \mathrm{~km}$, desde el Partido de Magdalena hasta Trenque Lauquen. Con la ubicación de los sitios de muestreos y determinaciones de laboratorio, las muestras se asociaron con series descriptas en cartas de suelos. Con el criterio estadístico de grupos de $\mathrm{K}$ media se formaron tres grupos de análisis. El Grupo 1, compuesto por suelos franco y franco arenosos, el Grupo 2 por areno franco y el Grupo 3 por franco limosos y franco arcillo-limosos.

Se realizaron determinaciones de la DTP sin materia orgánica por el método de la pipeta tradicional y determinaciones de la DTM sobre muestras sin dispersar con materia orgánica. De la curva de DTM se obtuvo la textura aparente formada por los porcentajes correspondientes a los microagregados tamaño arena, limo y arcilla.

Los resultados de las DTP fueron contundentes: Los suelos del G1 presentaron un comportamiento fractal intermedio, puesto que fue necesario recurrir a dos modelos fractales 
para explicar la DTP hasta el $95 \%$ de la masa total. El G2 reunió a los suelos no fractales y el G3 a los suelos con DTP fractal donde un solo modelo describe hasta el $98 \%$ de la masa. La masa acumulada de las arenas con diámetro superior a $150 \mu \mathrm{m}$ mostró un comportamiento no fractal en todos los casos. Las dimensiones fractales de fragmentación (D) de los Grupos 1 y 3 no se diferenciaron estadísticamente mientras que el factor de proporcionalidad, el parámetro $\mathrm{C}$, si mostró diferencias entre los Grupos. Como resultado, no citado hasta el momento, el valor de C fue considerado esencial para definir el modelo fractal de los suelos de cada Grupo. La D brinda información sobre el escalamiento, pero esta información es incompleta para definir un suelo y es imprescindible contar con el valor de $\mathrm{C}$.

Otro aporte, fue el desarrollo de un modelo para estimar los parámetros fractales $D$ y $C$ a partir de solo dos datos, el porcentaje de arena y arcilla. Los parámetros así estimados no se diferenciaron de los obtenidos por la vía experimental.

La microestructuración de las partículas por acción de la materia orgánica resultó de tipo fractal. Las diferencias entre las áreas de las curvas de DTM y la DTP fueron significativas. Se propuso un índice de microestructuración integrando los modelos fractales de partículas y microagregados.

El efecto de la materia orgánica sobre la fracción mineral en los tres Grupos fue similar, incrementó el porcentaje de la fracción de arenas aparentes respecto de la real, mientras que los porcentajes de limos y arcillas aparentes fueron menores que los reales. Estos resultados fueron confirmados con las fotografías tomadas a través de un microscopio óptico.

La relación entre las fracciones aparentes y la materia orgánica fue diferente en cada Grupo analizado. Los Grupos 1 y 2 no mostraron relación entre ambas variables. El Grupo 3 en cambio, mostró relaciones significativas con la materia orgánica. En los suelos del G3, el estado de microestructuración dependió de los contenidos de materia orgánica del suelo.

Se desarrolló un modelo teórico, donde es posible explicar la DTP de los suelos a partir de un Material Generador de naturaleza fractal, el transporte eólico y la sedimentación diferencial del material original. Con este modelo se puede explicar que a partir de un Material Generador de naturaleza fractal es esperable encontrar suelos fractales (como los del G3) formados con el material original más fino; suelos de naturaleza fractal intermedia, donde la pérdida de partículas finas hace necesario aplicar dos modelos para describirlos, y suelos de naturaleza no fractal (G2) los que se transformaron en suelos residualmente más gruesos. Los resultados experimentales obtenidos son consistentes con el modelo propuesto. Al tener igual " $D$ " se asume que pertenecen al mismo Material Generador, y las diferencias se observan solamente en el parámetro $C$ con valores medios de 19,2 en el G3 y 10,2 en el G1.

Se concluye que el proceso de microestructuración entre partículas minerales y materia orgánica, modificó la textura aparente favoreciendo el funcionamiento del sistema suelo, y que 
la DTP de los suelos de la región Pampeana deriva de un Material Generador fractal. Situaciones locales de materiales originales con DTP fractales, DTP de fractalidad intermedia o no fractales, son consecuencia del proceso eólico de transporte y depósito sobre este Material Generador. 


\section{Summary}

The soil particle size (SPS) is the most stable physical property of a soil and its knowledge is essential because it is related to the fertility, soil handling and taxonomic classification. On the other hand, the SPS is the main ingredient to the edaphology transfer equations. The SPS can be fully quantified with the particle size distribution (PSD) analysis or briefly through the texture calculation where only the total percentage of sand, clay and silt are considered. Nowadays cartographic surveys include intermediate values within the silt fraction from 2 to $20 \mu \mathrm{m}$ and very fine sand from 50 to $75 \mu \mathrm{m}$.

The fractal model has been used as a theoretical frame to describe the PSD of soils. A fractal object is formed by similar superimposed structures that can be seen at every level of detail. This property is known as self-similarity. On a fractal object the material is distributed on a diverse manner but not randomly and, statistically speaking; the generation pattern is identical and independent of the amplification factor. On a mathematical fractal the details of the pattern are exactly the same at every amplification level while in a natural fractal the repetition pattern is expected to be seen up to certain levels of amplification.

This work assumed as hypothesis that PSD and micro-aggregates of temperate climate rolling pampas soils have a fractal distribution. Information about the condition of micro-structures of a soil can be obtained thru the particles and micro-aggregates distribution analysis and fractal fragmentation analysis. The targets of this work were to investigate the fractal behavior of the soils PSD, the micro-aggregates size distribution (MSD) and the connection between soils PSD and the original material, process of transportation and sedimentation.

This work considered the PSD and MSD on soil samples of a $500 \mathrm{~km}$ transect within Buenos Aires province, from Magdalena to Trenque Lauquen counties. Knowing the samples location and after laboratory analysis samples were classified using soil charts. Three analysis groups were configured using a statistics criteria cluster K-media, the variables. Group 1 including loam and sandy loam, Group 2 loamy sand and Group 3 silty loam and silty clay loam.

The PSD without organic matter was determined by the pipet method and the MSD was calculated on samples with no scattered organic matter. The apparent texture, which is the percentage of micro-aggregates size sand, silt and clay, was established from the MSD curve.

The results were crystal clear: Group 1 soils have an intermediate fractal behavior as they required two fractal models to explain up to $95 \%$ of the total mass. Group 2 was no fractal type soils and Group 3 was fractal soils as they required only one model to describe up to $98 \%$ of the total mass. The mass of sands accumulated with particles diameter bigger than $150 \mu \mathrm{m}$ always behaved as non-fractal. Statistically speaking the fractal dimension (D) of Group 1 and 3 had no difference while the proportional factor $(C)$ was different on each group. As a result never quoted 
before, the factor $\mathrm{C}$ is considered essential in order to define the fractal model of each soil group. Fractal dimension $\mathrm{D}$ provides information about the fractal scaling factor but this parameter is not enough and factor $\mathrm{C}$ must be included in order to get a full definition of the soils.

Another contribution was the development of a model to calculate fractal parameters $D$ and $\mathrm{C}$ with only two figures which are the clay and sand percentage of a soil samples. There was no difference between parameters $C$ and $D$ obtained with the model and the same ones determined using soil samples in laboratory.

The particles micro-structured due to organic matter effect was well described by the fractal model. The difference between the areas of PSD and MSD curves was relevant. This work proposes a micro-structure index considering together the fractal particle model and the fractal micro-particles model.

The organic matter effect onto the mineral fraction on all the three analysis groups was similar: There was an increase of the apparent sand percentage vs the real one and the apparent silt and clay percentage was lower than the real one. These results were confirmed with photos taken thru an optical microscope.

The relationship between the apparent fraction and the organic matter was different on each analysis group: Groups 1 and 2 showed no connection at all meanwhile Group 3 showed very significant relationships with the organic matter. On Group 3 soils the micro-structure is dependent upon the soil organic matter content.

With the theoretical model developed it is possible to explain the PSD of soils coming from a fractal nature Material Generator, the wind transport and the differential sedimentation of the original material. This model is able to explain that starting with an fractal nature Material Generator it is expected to find fractal soils (like Group 3) with the finest original material; intermediate fractal soils, where the loss of fine particles need two models to explain the PSD and non-fractal soils (like group 2) which became coarse residual soils. The experimental results obtained in laboratory are consistent with the proposed model. Having the same parameter $D$ it is assumed that soils come from the same Material Generator and the differences can be observed only on the parameter $C$ with average values of 19.2 on Group 3 and 10.2 on Group 1.

As a conclusion, the micro-structures process between mineral particles and organic matter modified the apparent texture improving the soil system functioning and the PSD of the rolling Pampas come from a fractal Material Generator. Certain local conditions with original materials with full fractal PSD or intermedia-fractal PSD or non-fractal are a consequence of wind transport and deposit process onto this Material Generator. 
Índice general

1 Introducción general $1-1$

La distribución de tamaño de partículas en el suelo

1.1 Cuantificación de la distribución de tamaño de partículas $1-3$

1.2 El modelo fractal, generalidades ...................................................... 1-4

1.3 El modelo fractal aplicado al estudio de la DTP ......................................... 1-6

1.4 Estado del modelado fractal a la distribución de tamaño de partículas en la Ciencia del Suelo 1-9

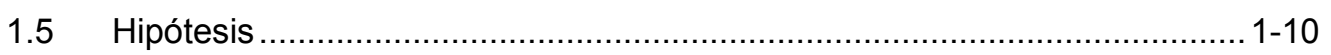

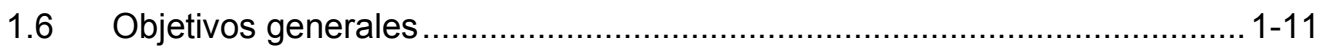

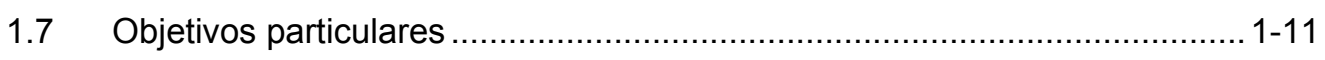

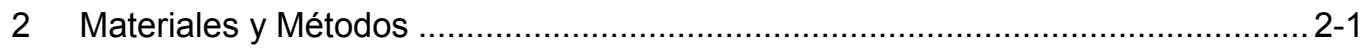

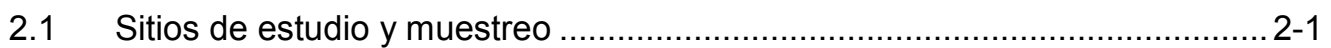

2.2 Tratamiento de las muestras y determinaciones de laboratorio .................... 2-1

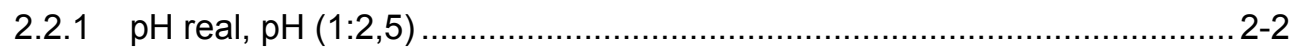

2.2.2 Capacidad de Intercambio catiónico (CIC) ....................................... 2-2

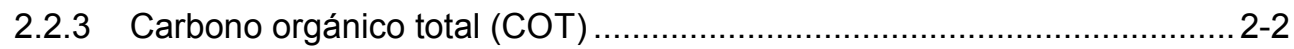

2.2.4 Carbono orgánico particulado ……..................................................... 2-3

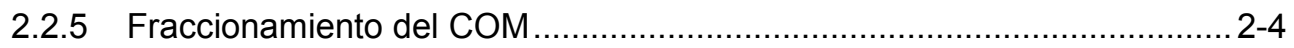

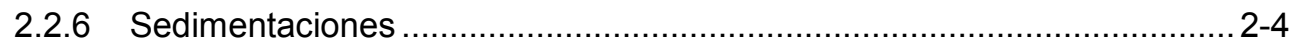

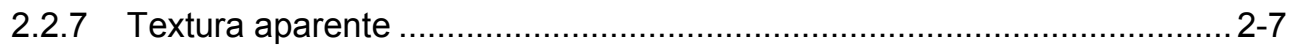

2.2.8 Fracciones sin materia orgánica y con materia orgánica, comparación directa $\quad 2-7$

2.2.9 Relaciones entre las fracciones sin materia orgánica y con materia orgánica $2-7$ 
2.2.10 Delta de las fracciones granulométricas .............................................. 2-7

2.2.11 Modelo fractal aplicado a la distribución de tamaño de partículas .........2-8

2.2.12 Escala sedimentológica. Parámetro Phi y calificaciones .......................2-9

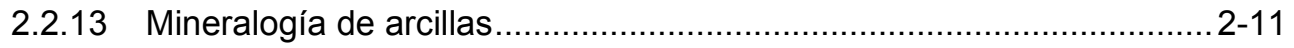

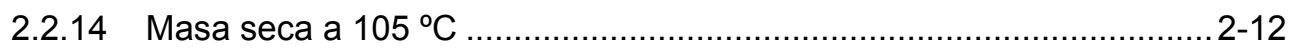

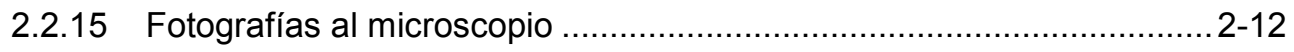

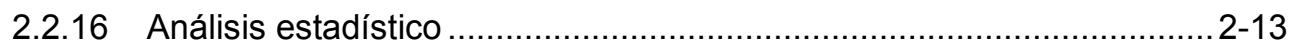

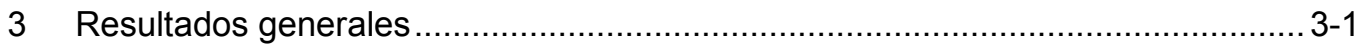

3.1 Características de los suelos estudiados ................................................ 3-1

3.1.1 Asociación de los sitios estudiados a series de suelos ............................ 3-1

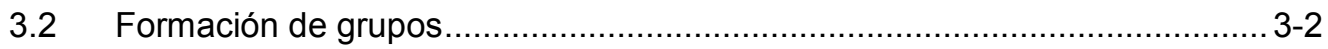

3.3 Características de los grupos ..............................................................

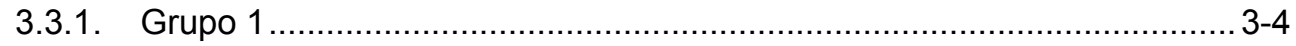

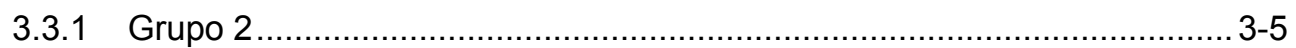

3.3.2 Grupo 3 …

3.4 Relación entre el carbono orgánico asociado a la fracción mineral y el carbono orgánico total 3-6

3.5 Carbono orgánico asociado a la fracción mineral, en función del contenido de arcilla y arcilla más limo para los tres grupos 3-6

3.6 Fracciones granulométricas reales y aparentes 3-9

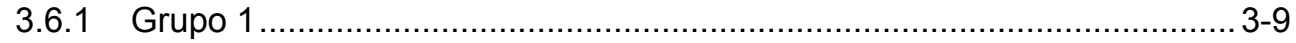

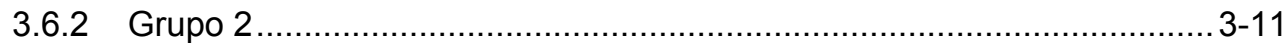

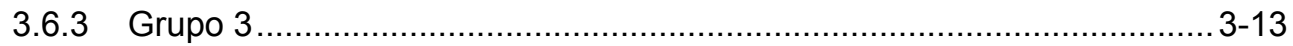

3.7 Estudio del carbono orgánico particulado y fraccionamiento del carbono orgánico asociado a la fracción mineral 3-16 
3.8 Análisis de la microestructuración en el tamaño arena........................ 3-18

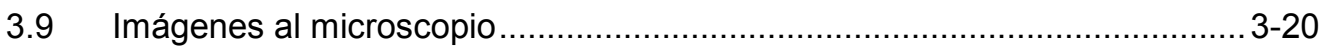

3.10 Discusión, fracciones reales y aparentes, microagregación ........................ 3-26

3.11 Conclusiones sobre la microestructuración ................................... 3-30

3.12 Alcance de la metodología y propuesta de futuras investigaciones ............3-30

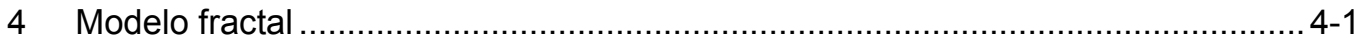

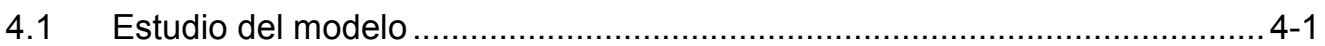

4.2 Información que brinda el modelo ............................................... 4-1

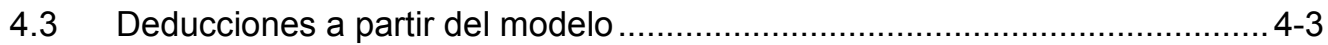

4.3.1 Funciones de igual dimensión fractal (Iso D) e igual diámetro máximo (Iso

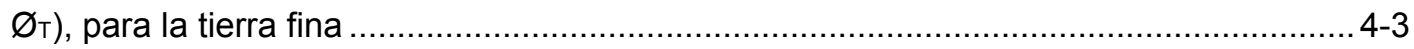

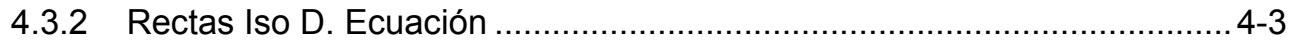

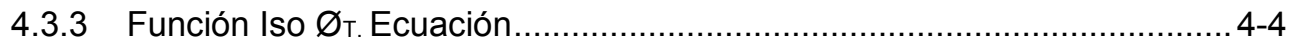

4.3.4 Ecuación para calcular la dimensión fractal teórica D ......................... 4-4

4.3.5 Parámetros del modelo fractal en función del contenido de arcilla..........4-5

4.4 Estudio fractal de la distribución de tamaño de partículas de los suelos muestreados 4-6

4.5 Grupo 1

4.5.1 Subdivisión del G1 en G1 Franco - G1 Franco Arenoso........................ 4-7

4.5.2 Grupo 1 franco. Análisis grupal ................................................... 4-8

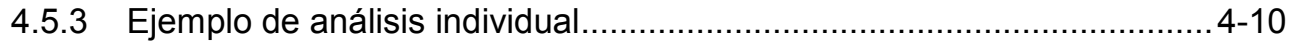

4.5.4 Grupo 1 franco arenoso. Análisis grupal .................................... 4-12

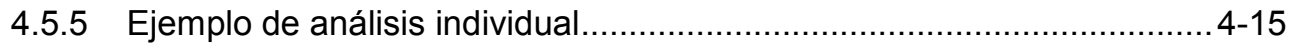

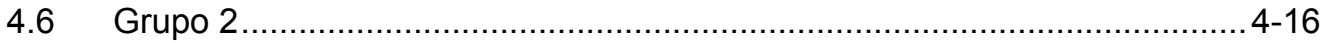


4.7.1 Ejemplo de análisis individual.

4.8 Análisis del modelo fractal de fragmentación experimental y teórico

4.8.1 Comparación de los parámetros fractales entre Grupos

4.8.2 Síntesis sobre el comportamiento fractal de las distribuciones de tamaño de partículas, sin materia orgánica 4-24

4.9 Discusión del modelo fractal sin materia orgánica 4-25

4.10 Conclusiones 4-28

5 Estudio fractal de la distribución de tamaño de microagregados 5-1

5.1 Resultados de las sedimentaciones con materia orgánica

5.2 Grupo 1. CMO. Análisis grupal 5-1

5.2.1 Ejemplo de análisis individual.

5.3 Grupo 2. CMO $5-4$

5.4 Grupo 3. CMO. Análisis grupa 5-5

5.4.1 Ejemplo de análisis individual. $5-6$

5.5 Discusión del análisis fractal sobre muestras con materia orgánica $5-8$

5.6 Comparación grupal SMO - CMO 5-9

5.7 Conclusiones del estudio fractal de las curvas de masa acumulada de microagregados 5-10

6 Propuestas 6-1

6.1 Validación del modelo fractal empleando datos de suelos de otras regiones descriptos por INTA

6.1.1 Introducción 6-1

6.1.2 Materiales y métodos

6.1.3 Resultados y discusión. 
6.1.4 Conclusión

6.2 Alcances y propuesta de trabajos a futuros

6.3 Modelización de la distribución de tamaños de partículas en la Pradera Pampeana 6-6

6.3.1 Modelización fractal a partir de un hipotético Material Generador 6-6

6.3.2 Tasas variables de transporte 6-9

6.3.3 Conclusión $6-11$

6.4 Modelización de la distribución de tamaños de partículas en la Pradera Pampeana, con los datos experimentales

6.5 Conclusión. 6-14

6.5.1 Conclusión de los datos experimentales $6-15$

7 Recapitulación

7.1 Hipótesis 7-1

Conclusión: $7-1$

Conclusión: 7-1

Conclusión:

7.2 Objetivos

7.3 Generales

7.4 Particulares.

7.5 Objetivos particulares.

7.6 Aplicaciones .

7.7 Futuras líneas de investigación derivadas de la tesis 7-5

7.7.1 Como aplicaciones, futuras se podría mencionar . 
9 Anexo 
Índice de Figuras

Figura 1.1. Triángulo textural propuesto por el USDA y las 12 clases texturales........1-2

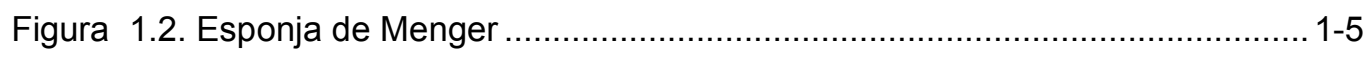

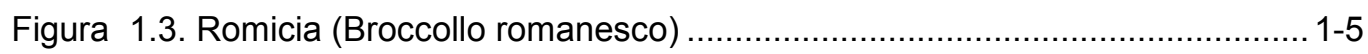

Figura 1.4. Modelo jerárquico de agregados Currie (1996) ................................ 1-6

Figura 1.5. Modelo de fragmentación de un cubo sólido propuesto por Turcotte, (1986). Se muestran las tres primeras etapas: 0, el cubo de arista $h$ se divide idealmente en ocho de aristas $\mathrm{h} / 2 ; 1$, el cubo de arista $\mathrm{h} / 2$ se divide ahora en ocho de aristas $\mathrm{h} / 4 ; 2$, el cubo de arista $\mathrm{h} / 4$ se divide en ocho de aristas $\mathrm{h} / 8$, y así siguiendo hasta el infinito.

Figura 2.1. Mapa con los sitios de muestreo georreferenciados, cada punto representa las muestras del tratamiento bajo alambrado y agrícola. 2-1

Figura 3.1. Proporciones medias de arena limo y arcilla por grupo. Distribución de tamaño de partículas de masa acumulada en función de Phi.

Figura 3.2. Relación del carbono orgánico particulado asociado a la fracción mineral (COM) en función del carbono orgánico total (COT), valores experimentales y recta de regresión.

Figura 3.3. Carbono orgánico particulado asociado a la fracción mineral (COM), separados por tratamientos "bajo alambrado = 0" y "agrícola = 1" y las correspondientes rectas de regresión, a, en función del contenido de arcillas y b en función del contenido de arcilla + limo.

Figura 3.4. Carbono orgánico total (COT) y carbono orgánico particulado, grueso (COPG), fino (COPF) y asociado a la fracción mineral (COM). Muestras bajo alambrado (0) y muestras agrícola (1). Las comparaciones son de a pares $0-1$, medias con una letra común no son significativamente diferentes $(p>0,05)$.

Figura 3.5. Fracciones texturales para el G1 según pretratamientos SMO y CMO. Relaciones entre fracción real y aparente. Variación relativa, Delta de cada fracción. Fracciones aparentes de arena, limo y arcilla en función del COM. Medias para la misma fracción, con una letra común no son significativamente diferentes $(p>0,05)$.

Figura 3.6. Fracciones texturales para el G2 según pretratamientos SMO y CMO. Relaciones entre fracción real y aparente. Variación relativa, Delta de cada fracción. Fracciones aparentes de arena, limo y arcilla en función del COM. 3-12 
Figura 3.7. Carbono orgánico total (COT) y carbono orgánico particulado (COP) de la fracción gruesa (COPG), COP de la fracción fina (COPF) y carbono orgánico asociado a la fracción mineral (COM) separadas por "0" bajo alambrado y "1" agrícola. Las comparaciones son de a pares $0-1$, medias con una letra común no son significativamente diferentes $(p>0,05) \ldots 3-$ 13

Figura 3.8. Fracciones texturales para el G3 según pretratamientos SMO y CMO. Relaciones entre fracción real y aparente. Variación relativa, Delta de cada fracción. Fracciones aparentes de arena, limo y arcilla en función del COM. Medias, para la misma fracción, con una letra común no son significativamente diferentes $(p>0,05)$.

Figura 3.9 a. Carbono orgánico total (COT) expresado por la suma de las fracciones de carbono orgánico particulado (COP). b Proporción relativa de las fracciones de COP en relación al COT. 3-17

Figura 3.10a Valor absoluto $\left(\mathrm{g} \mathrm{kg}^{-1}\right)$ del carbono orgánico asociado a la fracción mineral (COM) expresado como el valor acumulado de las fracciones de 2, 20 y $50 \mu \mathrm{m}$. b Proporción relativa de las fracciones del COM respecto del carbono orgánico total (COT).

Figura 3.11a. Carbono orgánico total (COT) expresado por la suma de las fracciones de carbono orgánico particulado (COP) y las subdivisiones del COM. b Proporción relativa de las fracciones del COP y las subdivisiones del COM en relación al COT. 3-18

Figura 3.12 Cociente entre los valores de carbono orgánico aportado por cada fracción, entre el suelo bajo alambrado (BA) y el agrícola (Agríc) 3-18

Figura 3.13.a Valores de arena aparente en función del carbono orgánico asociado a la fracción mineral (COM), para los Grupos 1, 2 y 3. Relación Arenas / Arenas aparentes en función del COM. 3-19

Figura 3.14.-Fotos tomadas sobre las arenas. La columna de la izquierda corresponde al S1, la del medio $\$ 29$ y la derecha al S9.

Figura 3.15.-Fotos tomadas sobre la suspensión de limos y arcillas. La columna de la izquierda corresponde al S1, la del medio S29 y la derecha al S9.

Figura 3.16.-Fotos tomadas sobre la suspensión CMO. La columna de la izquierda corresponde al S1, la del medio S29 y la derecha al S9.

Figura 3.17. Relaciones entre las fracciones reales y aparentes para los tamaños arena, limo y arcilla. Comparación del valor de Phi para los Grupos 1, 2 y 3 según el tratamiento sin materia orgánica $(\mathrm{SMO})$ y con materia orgánica $(\mathrm{CMO})$ 3-29 
Figura 3.18. Relación entre fracciones aparentes de arena limo y arcilla en función del COM (a) para el G1 y (b) para el G3 3-30

Figura 4.1. Triángulo textural, con las clases texturales tradicionales USDA. Funciones Iso D (---) e Iso $\varnothing_{\text {T. }}(-\cdot-)$.

Figura 4.2. a. Dimensión fractal de fragmentación " $D$ " en función del contenido de arcilla para diferentes $\emptyset_{\text {T. }}$ b Valor de C en función del contenido de arcillas para diferentes "D", ambas expresiones deducidas del modelo teórico.

Figura 4.3, a. Distribución de tamaño de partículas completa del G1, de masa acumulada en función del diámetro, ambos ejes en escala logarítmica; b. Distribución de tamaño de partículas entre $2-75 \mu \mathrm{m}$, ambos ejes en escala logarítmica; c. Distribución de residuos 4-7

Figura 4.4. Gráfico de masa acumulada en función del parámetro Phi para el G1 y los resultantes G1F y G1FA, luego de la separación. 4-8

Figura 4.5. Distribución de tamaño de partículas de masa acumulada en función de Phi.

Figura 4.6. Modelo fractal para el G1F, acotado por sectores, valores experimentales observados en la distribución de tamaño de partículas, masa acumulada en función del diámetro.

Figura 4.7. Modelo fractal acotado por sectores, ajustados particularmente al suelo S26 del G1F, Hapludol éntico serie Bolívar, Valores experimentales observados en la distribución de tamaño de partículas, masa acumulada en función del diámetro.

Figura 4.8. Distribución de tamaño de partículas de masa acumulada en función de Phi para el G1FA.

Figura 4.9. Modelo fractal para el G1FA, acotado por sectores, valores experimentales observados en la distribución de tamaño de partículas, masa acumulada en función del diámetro.

Figura 4.10. Modelo fractal acotado por sectores, ajustados particularmente al suelo S9, valores experimentales observados en la distribución de tamaño de partículas, masa acumulada en función del diámetro. 4-16

Figura 4.11. Distribución de tamaño de partículas para el G2, ambos ejes en escala logarítmica 4-17

Figura 4.12. Gráfico de residuos para el modelo fractal del G2. $4-18$ 
Figura 4.14.a. Modelo lineal propuesto para el G2. b Modelo Fractal, descartada para explicar la DTP en este Grupo de suelos

Figura 4.15. Modelo fractal para el G3, acotado por sectores, valores experimentales observados en la distribución de tamaño de partículas, masa acumulada en función del diámetro.

Figura 4.16. Modelo fractal acotado por sectores, ajustados particularmente al suelo S3. Valores experimentales observados en la distribución de tamaño de partículas, masa acumulada en función del diámetro. 4-22

Figura 4.17. Comparación entre valores experimentales "E" obtenidos de las curvas de sedimentación y valores teóricos " $T$ " obtenidos con las Ecuaciones 4.10 y 4.11 para los parámetros $\mathrm{C}$ y $\mathrm{D}$

Figura 4.18. Comparación de los parámetros fractales C y D por grupo. Medias con una letra común no son significativamente diferentes $(p>0,05)$.

Figura 4.19. Valores de D experimental en función del contenido de arcilla \%. Se representaron como referencia en líneas punteadas las curvas teóricas correspondientes a D para $\emptyset_{T}=60$, (línea inferior), $\varnothing_{T}=100 \mu \mathrm{m}$ (línea media) y $\emptyset_{T}=1000 \mu \mathrm{m}$ (línea superior) .... 4-26

Figura 4.20. a Relación lineal de "C" en función del contenido de arcillas expresadas en porciento. b Gráfico de residuos del modelo lineal propuesto.

Figura 5.1a. Curva comparativa de masa acumulada en función de Phi para G1FA-G1 y G1F. b fracciones texturales aparentes para el G1 y c curva de masa acumulada en función de Phi para el G1. 5-1

Figura 5.2a. Distribución de los residuos para el modelo. b. Datos experimentales y recta de regresión ajustada (CMO).

Figura 5.3a. Proporciones de arena limo y arcillas aparentes para el S24CMO. b Curva de masa acumulada en función de Phi para el S24.

Figura 5.4. Distribución de tamaño de microagregados (CMO) en función del diámetro $\varnothing$ $(\mu \mathrm{m})$. Modelo fractal propuesto.

Figura 5.5 Distribución de tamaño de microagregados (CMO) en función del diámetro $\varnothing$ $(\mu \mathrm{m})$. Modelo no fractal propuesto. 
Figura 5.6. Distribución de tamaño de microagregados (CMO), para el G3, en función del diámetro $\varnothing(\mu \mathrm{m})$. Modelo fractal propuesto. 5-6

Figura 5.7a. Proporciones de arena limo y arcillas aparentes para el G3CMO. b Curva de masa acumulada en función de Phi para el mismo Grupo.

Figura 5.8. Distribución de tamaño de microagregados en función del diámetro $\varnothing(\mu \mathrm{m})$. Modelo fractal propuesto.

Figura 5.9. Valores de D (SMO), cuando el contenido de tamaño arcilla $>5 \%$. Cuando el contenido de tamaño arcilla es $<5 \%$, corresponde a las $\mathrm{D}(\mathrm{CMO})$. 5-9

Figura 5.10 G1FA corresponde al grupo 1 franco arenoso analizando las partículas, G1F corresponde al grupo 1 franco arenoso SMO, G1CMO corresponde a la curva de microagregados. G3 corresponde al grupo 3 analizando las partículas, G3CMO corresponde a la curva de microagregados. $5-10$

Figura 6.1. Asociación entre los valores experimentales y los valores calculados por el modelo propuesto para el suelo de la serie Arrecifes.

Figura 6.2. MG Material Generador de naturaleza fractal. SPA suelo de la Pampa Arenosa, enriquecido residualmente por fracciones gruesas. SPA(F) modelo fractal parcial hasta el $39,9 \%$ de la masa, entre 2 y $75 \mu$ m para SPA. SPO suelo de la Pampa Ondulada, de naturaleza fractal entre 2 y $75 \mu \mathrm{m}$

Figura 6.3. DTP teórica de suelos resultantes de considerar tasas variables de transporte eólico. $6-11$

Figura 6.4. Distribución de tamaño de partículas del Material Generador resultante de integrar las partículas de los Grupos de suelos G1F, G1FA, G2 y G3. $6-13$ 
Índice de Tablas

Tabla 2.1 Esquema de tiempos necesarios para obtener las fracciones acumuladas de diámetro aparente $\varnothing(\mu \mathrm{m})$ en función de la profundidad de extracción $(\Delta y)$ y la temperatura de la suspensión.

Tabla 2.2 Equivalencia de diámetro $(\varnothing)$ en milímetros y el parámetro Phi $2-10$

Tabla 2.3. Calificación de las sedimentaciones a partir de los valores de la media, expresados en Phi y desvío estándar 2-10

Tabla 2.4. Calificación cuali cuantitiva de los resultados de las determinaciones mineralógicas por difracción de rayos $X$. (Comunicación personal Poire)...... 2-12

Tabla 3.1. Número de suelo, condición del sitio muestreado (BA) bajo alambrado, (Agríc) agrícola, la serie de suelo con la que fue asociado y la clasificación taxonómica (USDA, Soil Taxonomy). $\left(^{*}\right)$ Tapto es un localismo empleado en Argentina, no reconocido por USDA, Soil Taxonomy.

Tabla 3.2 Valores medios de los porcentajes de arena $(\mathrm{A} \%)$, arcilla (arc\%) y diámetro correspondiente a la media (Phi), expresado en $\mu \mathrm{m}$. Grupos 1, 2 y 3

Tabla 3.3. Detalle de los grupos, suelos que lo conforman, condición de muestreo y serie taxonómica asociada.

Tabla 3.4. Suelos empleados realizar el fraccionamiento del COM, Serie Pas: Piedritas, Bo: Bombeador, C.T clase textural FA: franco arenoso, FL: franco limoso. Condición del suelo BA: bajo alambrado, Agríc: agrícola. 3-16

Tabla 3.5. Valores de carbono orgánico ( $\mathrm{CO}$ ) medido sobre las diferentes fracciones de microagregados. El CO acumulado es lo porción CO que aporta al carbono orgánico total (COT)

Tabla 3.6 Valores de $\mathrm{p}$ en el cuerpo de la tabla para las fracciones aparentes y las diferentes fracciones de carbono analizadas.

Tabla 6.1 Provincia a la que pertenecen las Series analizadas, horizonte estudiado (Hte), A (\%) porcentaje de arena, $L(\%)$ porcentaje de limo y arc (\%) porcentaje de arcilla. C parámetro del modelo fractal estimado, $D$ dimensión fractal de fragmentación estimada, $\varnothing_{\mathrm{T}}$, diámetro máximo teórico esperable y $\mathrm{R}$ coeficiente de correlación

Tabla 6.2. Distribución de tamaño de partículas, con una tasa de transporte eólico del 80\% para el Material Generador (MG), suelo de la Pampa Arenosa (SPA), modelización fractal del suelo de la Pampa Arenosa (SPA(F)) y del suelo de la Pampa Ondulada (SPO).... 6-8 
Tabla 6.3. Distribución de tamaño de partículas acumulado expresado en porcentaje, con tasas variables de transporte eólico. $\varnothing$ : diámetro de partículas en $\mu \mathrm{m}$. MG, Material Generador; SPA suelo de la Pampa Arenosa; SPA(F) suelo de la Pampa arenosa estimada con el modelo fractal; SPO suelo de la Pampa Ondulada.

Tabla 6.4 Distribución de tamaño de partícula en porcentaje acumulado del MG Material Generador, G1F Grupo 1 de suelos franco, G1FA Grupo 1 de suelos franco arenosos, G2 Grupo 2 y G3 Grupo 3 6-13 


\section{Introducción general}

Capítulo 1 


\section{Introducción general}

La distribución de tamaño de partículas en el suelo

La distribución del tamaño de partículas (DTP), es una de las características más estables en un suelo. El interés en su determinación, radica en que está relacionada con otras propiedades físicas y químicas, como ser: porosidad, interacción con los fluidos y solutos, compresibilidad y comportamiento térmico, capacidad de intercambio catiónico, laboreo, susceptibilidad a la erosión y desertificación, curvas de retención hídrica, conductividad hidráulica saturada e insaturada, composición química y crecimiento de las plantas, entre otras ( Arya y Paris, 1981; Campbell y Shiozawa, 1992; Porta Casanellas, 1999; Vdovic et al. 2010). Se usa también como insumo en la estimación teórica de propiedades hidrológicas de suelos, a través de las funciones de transferencia edafológica (Pachepsky y Rawls, 2004).

La determinación de la DTP es uno de los análisis más comunes e importantes en la Física de Suelos. Es usado en análisis texturales para la clasificación de suelos, con propósitos agronómicos e ingenieriles. Como se dijo, tiene también una relación directa e indirecta con la distribución de poros en los suelos y, por tanto, con las propiedades de retención de humedad y movimiento del agua. En su versión más simple, se determinan las fracciones de arena, limo y arcilla que contiene una muestra de suelo. En este análisis se determina la textura del suelo. Antiguamente, se determinaba por el tacto en forma directa, mediante una rutina que incluía desmenuzar entre los dedos una muestra de suelos, mediante las sensaciones que producía el amasado de la muestra húmeda en las manos.

Existen varias clasificaciones de suelos elaboradas por distintas instituciones internacionales, que se diferencian en los límites de los diámetros de las partículas que forman el suelo. 
A modo de ejemplo se presentan las siguientes:

Fracciones granulométricas

\begin{tabular}{llc}
\hline \multirow{2}{*}{$\begin{array}{c}\text { Granulometría } \\
\text { denominación }\end{array}$} & Fracciones \\
\cline { 2 - 3 } & Denominación & Diámetros aparentes $(\mu \mathrm{m})$ \\
\hline USDA Simple & Arena USDA & $50<\varnothing<2000$ \\
& Limo USDA & $2<\varnothing<50$ \\
& Arcilla & $\varnothing<2$ \\
\hline USDA & Arena gruesa USDA & $500<\varnothing<2000$ \\
& Arena fina USDA & $50<\varnothing<500$ \\
& Limo USDA & $2<\varnothing<50$ \\
& Arcilla & $\varnothing<2$ \\
\hline USDA Completa & Arena muy gruesa & $1000<\varnothing<2000$ \\
& Arena gruesa & $500<\varnothing<1000$ \\
& Arena media & $250<\varnothing<500$ \\
& Arena fina & $100<\varnothing<250$ \\
& Arena muy fina & $50<\varnothing<100$ \\
& Limo & $2<\varnothing<50$ \\
& Arcilla & $\varnothing<2$ \\
\hline Internacional simple & Arena ISSS & $20<\varnothing<2000$ \\
& Limo ISSS & $2<\varnothing<20$ \\
& Arcilla & $\varnothing<2$ \\
\hline
\end{tabular}

Las combinaciones de estas fracciones han formado el triángulo textural (Figura 1.1), según el USDA (United States Department of Agriculture, Estados Unidos), que contempla 12 clases texturales. Los suelos dentro de estas se agrupan por similares características de manejo y comportamiento. La clase central es la "franco" la cual se define como la proporción de arena limo y arcilla que producen igual sensación al tacto, donde las tres fracciones se perciben por igual sin predominio de una sobre otra. Estas clases texturales tienen intervalos en los contenidos de cada fracción mientras que una DTP brindaría mayor información sobre la composición del suelo (Bitelli et al. 1999).

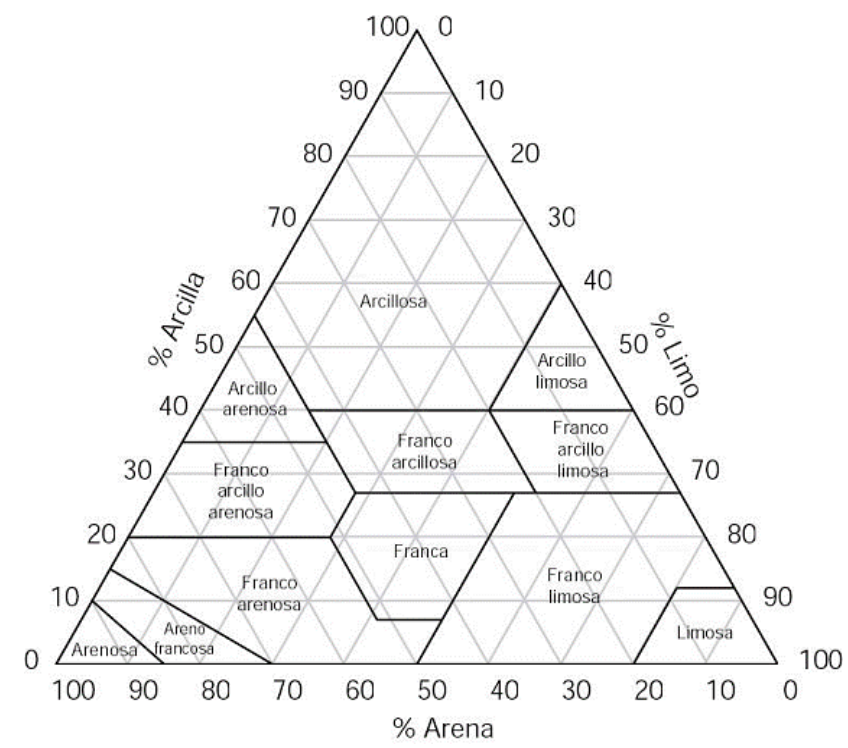

Figura 1.1. Triángulo textural propuesto por el USDA y las 12 clases texturales. 


\subsection{Cuantificación de la distribución de tamaño de partículas}

Existe una amplia gama de métodos que permiten llevar a cabo este tipo de análisis. Entre ellos tenemos: el del hidrómetro (densímetro), de la pipeta, del sensor de presión, de la atenuación de rayos $\mathrm{X}$, el de difracción de rayos láser y el de medición de la resistencia eléctrica de la suspensión de suelo en agua (Gee y Bauder, 1986, Gee y Or, 2002, Vdovic et al. 2010). Los resultados del análisis textural dependen del método de determinación y de la preparación de la muestra.

En nuestro país, uno de los métodos que se emplea con mayor regularidad es el del hidrómetro (conocido como Método de Bouyoucos). El mismo utiliza la ley de Stokes para interpretar los datos experimentales, que para el hidrómetro puede ser escrita como una dependencia lineal entre el diámetro de la partícula, $\varnothing(\mu \mathrm{m})$, y el inverso de la raíz cuadrada del tiempo de sedimentación del tipo:

$$
\emptyset=\frac{k}{\sqrt{t}} \quad \text { Ecuación 1-1 }
$$

Donde " $k$ " es una constante y "t" es el tiempo que tarda una partícula esférica, de densidad real 2,65 $\mathrm{g} \mathrm{cm}^{-3}$ en sedimentar en un líquido viscoso. Básicamente, el hidrómetro se coloca en una suspensión de suelo dispersado en agua, que se encuentra en una probeta, se agita cuidadosamente y se mide la variación de la densidad de la suspensión a través de la flotación del densímetro, en función del tiempo. De la información obtenida se determinan los porcentajes de arena, limo y arcilla que contiene el suelo estudiado (Gee y Bauder, 1986; SAMLA, 2004).

El otro método empleado con frecuencia y considerado de referencia, es el de la pipeta o Robinson. También utiliza la ley de Stokes para analizar la sedimentación de partículas de suelo en una probeta con agua, pero toma muestras de la suspensión mediante una pipeta a profundidades fijadas en tiempos determinados. Las porciones de suspensión extraídas son secadas y se pesa el componente sólido remanente (Gee y Bauder, 1986; SAMLA, 2004). De esta información se obtienen los porcentajes de arena, limo y arcilla. Más detalles en el capítulo correspondiente.

Es interesante mencionar aquí que ambos métodos utilizan pretratamientos del suelo diferentes, por lo que las DTP con las que se obtiene la clase textural son diferentes, aunque los resultados de clase textural pueden ser similares, según el tipo de suelo estudiado. Ambos métodos utilizan pocos datos recopilados en la sedimentación de la muestra.

Day (1965) y Gee y Bauder (1986), señalaron que solo si el suelo tiene un bajo contenido de materia orgánica y se encuentra libre de sales solubles, el método de Bouyoucos tiene un bajo error con respecto al método de la pipeta. 
Las diferencias y similitudes entre los mencionados métodos de análisis generaron preguntas que no tenían respuestas contundentes y fue uno de los motivos por lo que se decidió investigar más sobre las DTP y sus posibles asociaciones para formar microagregados 0 pseudopartículas de suelos.

Para abordar esta investigación se optó por emplear la teoría fractal siguiendo numerosos trabajos publicados al respecto en la ciencia del suelo.

¿Por qué emplear esta teoría para describir en principio la DTP y no limitarse a funciones matemáticas o estadísticas simplemente? Porque esta teoría tiene sustento físico y emplea pocos parámetros que simplifican la modelización.

\subsection{El modelo fractal, generalidades}

En los últimos años la geometría fractal (Mandelbrot, 1982) ha permitido describir teóricamente muchas de las estructuras que se observan en la naturaleza. Es así que las características geométricas de las nubes, la distribución espacial de las raíces y el desarrollo foliar en plantas, las corrientes turbulentas, las longitudes de las costas y fronteras, el fraccionamiento de una roca, la distribución de tamaño de partículas de algunos suelos, la formación de los cristales de nieve, el sistema venoso del cuerpo, los meandros de un río, entre otros, pueden ser idealizados satisfactoriamente mediante esta teoría (Korvin, 1992; Tyler y Wheatcraft, 1992; Filgueira et al. 2005; Prosperini y Perugini, 2008; Vdovic et al. 2010). Un objeto fractal está compuesto de una cantidad de estructuras similares superpuestas, visibles a cualquier nivel de detalle. Esta propiedad se conoce como autosimilitud. El material en un objeto fractal se distribuye en forma heterogénea, pero no al azar, de forma que un patrón de generación se ve idéntico, al menos en el sentido estadístico, independientemente de la amplificación con que se esté observando. En un fractal matemático los detalles se ven idénticos en cualquier nivel de amplificación mientras que en un fractal natural es esperable que el nivel de repetición de la propiedad particular sea limitado a varios órdenes de magnitud.

Un ejemplo es la Esponja de Menger, (Figura 1.2) que comienza con un cubo sólido, al cual se le aplica un proceso de extracción de partes, en cada paso similar al anterior, aplicado cada vez a cubos más pequeños. Esta secuencia se puede llevar al infinito. 


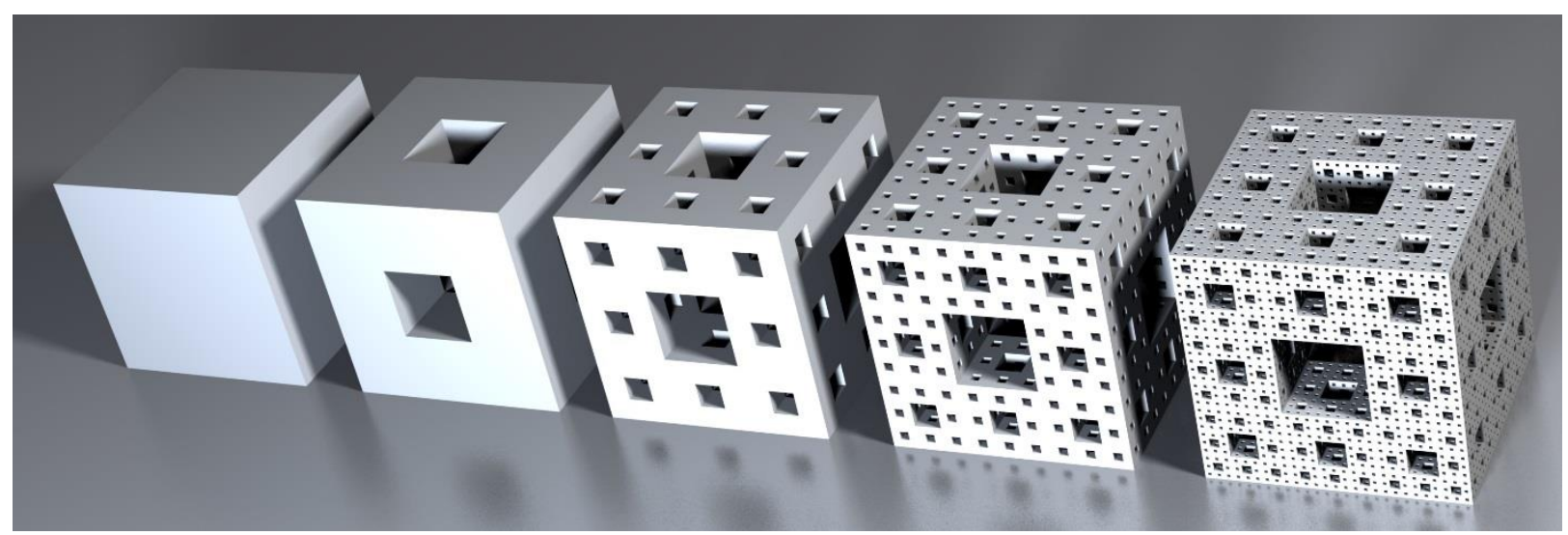

Figura 1.2. Esponja de Menger

Los pasos de construcción de estos volúmenes son:

Comenzamos con un cubo (primera imagen). Dividimos cada cara del cubo en 9 cuadrados. Esto subdivide el cubo en 27 cubos más pequeños. Eliminamos los cubos centrales de cada cara (6) y el cubo central (1), dejando solamente 20 cubos (segunda imagen). Repetimos los pasos 1, 2 y 3 para cada uno de los veinte cubos menores restantes. Repetimos el proceso para los cubos que vayan quedando. Este tipo de figuras geométricas fue durante cientos de años dejada de lado por los matemáticos y llegaron a llamarse "monstruos". Mandelbrot (1986) rescata muchas de estas figuras y las unifica en un concepto nuevo, de fractales matemáticos.

En la naturaleza tenemos ejemplos: el romanesco Brassica oleracea es un híbrido de brécol (Brassica oleracea var. itálica) y coliflor (Brassica oleracea var. botrytis) de la familia de las brasicáceas (Figura 1.3). El brécol romanesco fue documentado inicialmente en Italia (como Broccolo romanesco) en el siglo XVI. También se llama Romicia, el cual es otro ejemplo de fractal en la naturaleza.
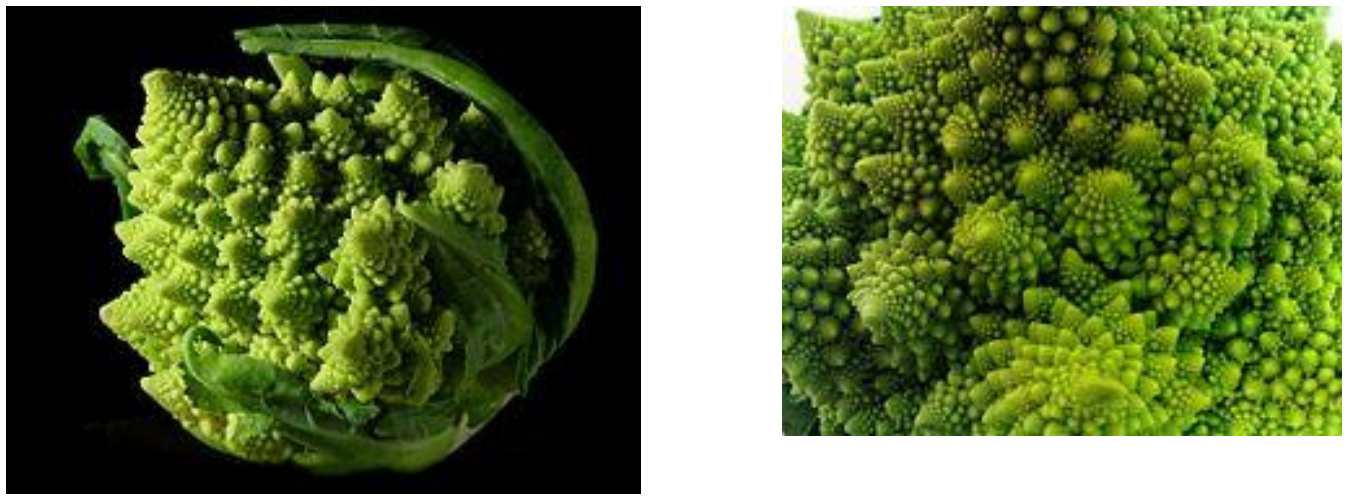
Una de sus más llamativas características es que presenta geometría fractal en su estructura. La cantidad de inflorescencias que compone el brécol romanesco es un número Fibonacci.

Un modelo de agregado fractal sería el siguiente:

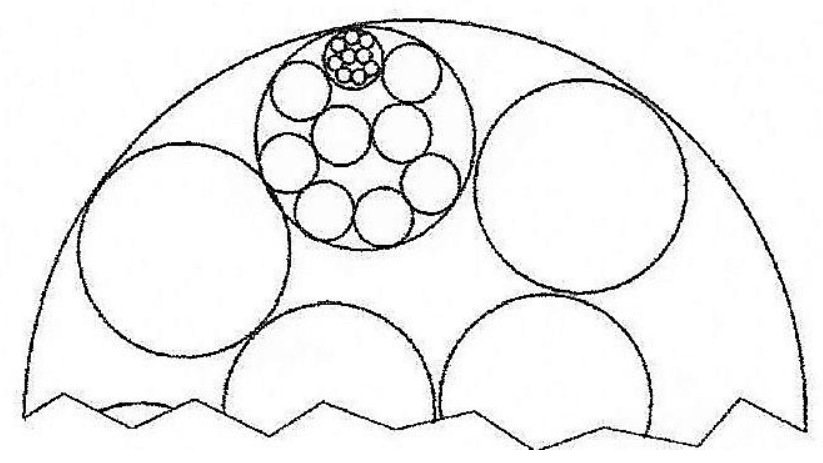

Figura 1.4. Modelo jerárquico de agregados Currie (1996)

Se ha discutido acerca del rango de validez de este marco teórico en los objetos reales (Avnir et al. 1998). La principal objeción es acerca del rango de aplicación de la propiedad de autosimilitud, que en vez de ser infinito como en el fractal matemático, sólo abarcaría uno o dos órdenes de magnitud en los fractales naturales. Sin embargo, el modelo geométrico de Mandelbrot ha logrado consolidarse en varias ciencias como adecuado.

\subsection{El modelo fractal aplicado al estudio de la DTP}

El modelo fractal de fragmentación intenta explicar la DTP observada con un conjunto mínimo de parámetros (Tyler y Wheatcraft, 1992), donde un detalle ampliado es semejante al todo, por lo que una figura fractal presenta autosimilitud (Vivas Miranda et al. 1999). Matemáticamente la autosimilitud conlleva a una relación potencial (Valdez Cepeda et al. 1998).

En la ciencia del suelo se ha utilizado la geometría fractal para arrojar nueva luz sobre la compleja dinámica de los procesos formadores del suelo mediante el estudio de las DTP (Tyler y Wheatcraft, 1992; Hyslip y Vallejo, 1997; Bittelli et al. 1999; Taguas et al. 1999; Perfect et al. 2002; Millán et al. 2003; Stanchi et al. (2006 y 2008)).

Turcotte (1986) ha trabajado sobre la fragmentación de un cubo sólido con probabilidad de rotura variable, introduciendo la dimensión fractal de fragmentación (D).

Una celda cúbica de orden cero, con dimensión h, se divide en 8 elementos cúbicos con dimensión h/2. La probabilidad de que una celda cúbica de orden cero sea fragmentada en 8 elementos es $p_{c}$. Los fragmentos con dimensión $\mathrm{h} / 2$ se transforman ahora en celdas de primer orden. Cada celda de $1^{\text {er }}$ orden se divide ahora en 8 elementos de dimensión $\mathrm{h} / 4$. La probabilidad 
de que una celda cúbica de $1^{\text {er }}$ orden se fragmente en 8 elementos es también $p_{c}$. El proceso se repite hacia órdenes mayores (Figura 1.5).

Aplicación: modelización del proceso de fragmentación Turcotte, (1986)

$$
\begin{aligned}
& D=\frac{\ln \left(8 p_{c}\right)}{\ln 2} \quad \text { Ecuación } 1-2 \\
& \frac{1}{8}<p_{c}<1 \\
& 0<D<3 \\
& N(r) \approx r^{D}
\end{aligned}
$$

La probabilidad máxima sería $p=1$, que implica una división en ocho cubos más chicos, y la mínima sería $p=1 / 8$, para el desprendimiento de un solo cubo de los ocho posibles, $\mathrm{N}$ es el número de cubos acumulado mayores que "r" y $\mathrm{D}$ la dimensión fractal de fragmentación.

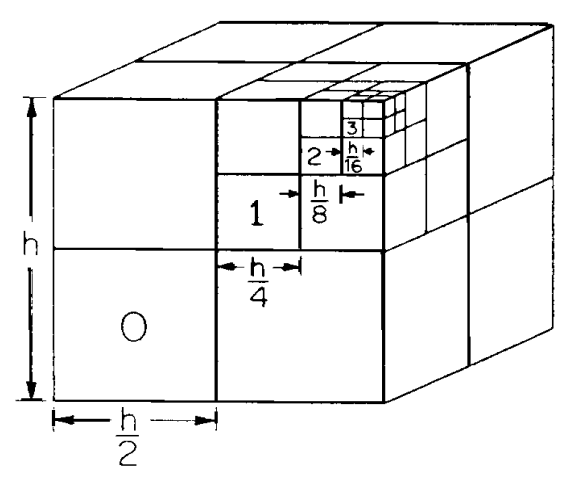

Figura 1.5. Modelo de fragmentación de un cubo sólido propuesto por Turcotte, (1986). Se muestran las tres primeras etapas: 0 , el cubo de arista $h$ se divide idealmente en ocho de aristas $h / 2 ; 1$, el cubo de arista $h / 2$ se divide ahora en ocho de aristas $h / 4 ; 2$, el cubo de arista $h / 4$ se divide en ocho de aristas $h / 8, y$ así siguiendo hasta el infinito.

Para la determinación de $D$ se sugirió la Ecuación 1.3, derivada de la teoría de la distribución fractal de objetos (Mandelbrot, 1982):

$$
N(r>R)=k(R)^{-D} \quad \text { Ecuación 1-3 }
$$

Donde " $D$ " es la dimensión fractal de fragmentación, $N(r>R)$ es el número de objetos acumulados mayores que un cierto tamaño " $R$ " y " $k$ " es una constante igual a $N(r>R)$ para $R=1$. El valor de $\mathrm{D}$ depende de la forma de los objetos individuales dentro de la distribución y de la fragmentación en cada uno de los niveles. En el caso de objetos regulares, cubos, por ejemplo, D 
es únicamente una medida de la fragmentación. Cuanto más grande es el valor de $D$, mayor es el grado de fragmentación. El valor de esta dimensión puede variar entre 0 y 3.

Este modelo se ha aplicado en Física de Suelos. El número de objetos fragmentados $\mathrm{N}(r>R)$, pueden ser agregados o partículas de suelo, se estima suponiendo algún tamaño característico $R_{n}$ cuyo volumen y densidad son usados para el cálculo de los objetos retenidos entre dos tamices (lo que pasó por uno y quedó retenido en el otro). Para esto se emplea la siguiente ecuación (Tyler y Wheatcraft, 1989):

$$
N\left(r>R_{n}\right) \approx \sum_{i=1}^{n} N\left(R_{1}<r<R_{i+1}\right)=\sum_{i=1}^{n} \frac{M\left(R_{i}<r<R_{i+1}\right)}{\frac{4}{3} \pi R^{3} \rho_{p}}=k R^{-D} \quad \text { Ecuación 1-4 }
$$

Donde $M\left(R_{i}<r<R_{i+1}\right)$ representa la masa de partículas o agregados entre dos tamices seguidos y " $\rho_{p}$ " es la densidad de las partículas. Este cálculo está muy afectado por la elección de $R_{n}$ (promedio), especialmente cuando este valor es pequeño, pues aparece al cubo en el denominador. Por último, considerar constante la densidad de las distintas fracciones, separadas por tamizado, puede llevar también a errores. Este tipo de modelo ha presentado, en muchos casos, resultados que no tenían sentido físico ( $D$ mayor que 3 ).

Tyler y Wheatcraft (1992), plantearon otra forma de enfocar el mismo problema. La alternativa sugerida fue considerar directamente la masa de partículas, en cambio del número de ellas. Esto conlleva ventajas, pues es experimentalmente más sencillo y preciso obtener fracciones de masa que número de partículas. En el modelo propuesto, la fracción de masa acumulada de un radio menor que un valor $R$ es (Tyler y Wheatcraft, 1992):

$$
\begin{aligned}
\frac{M(r<R)}{M_{T}} & =\left(\frac{r}{R_{L \text { sup }}}\right)^{3-D}=C r^{3-D} \quad \text { Ecuación 1-5 } \\
\log M_{(r<R)} & =\log C+(3-D) \log r
\end{aligned}
$$

Donde $M_{T}$ es la masa total de la distribución de las partículas, y $r=R_{L s u p}$ es un límite superior de tamaño para el comportamiento fractal y $C$ es una constante. Si se aplica logaritmo a ambos miembros de la ecuación, la dimensión fractal $D$ puede ser calculada de la pendiente de la regresión lineal. Este modelo resultó más razonable desde el punto de vista físico, con resultados de $\mathrm{D}$ siempre menores que 3 . La temática se ampliará en el capítulo de Materiales y Métodos. 
Filgueira et al. (2003), encontraron una relación entre la distribución de masa acumulada de partículas en sedimentación y el tiempo. Los autores estudiaron el caso de una DTP partiendo de la base de que la distribución es fractal, cuando la misma se encontraba cayendo en un líquido viscoso, de acuerdo con la ley de Stokes. Se encontró una relación explícita entre la fracción de masa de partículas en suspensión, el tiempo de sedimentación y la dimensión fractal de fragmentación. La ecuación, que expresa la relación mencionada, es:

$$
\frac{M_{\left(r<R_{L s u p}\right)}}{M_{T}}=k \times t^{\frac{D-3}{2}} \quad \text { Ecuación 1-6 }
$$

Donde k está conformada por $L$ y $B$ que son constantes, $R_{L \text { sup }}$ es el límite superior de radio para el cual se cumple el comportamiento fractal, $D$ es la dimensión fractal de fragmentación y $t$ es el tiempo. Si se aplica logaritmo a ambos miembros de la Ecuación. 1.6, la dimensión fractal D puede ser calculada de la pendiente de la Ecuación 1.6 linealizada con logaritmo.

\subsection{Estado del modelado fractal a la distribución de tamaño de partículas en la Ciencia del Suelo}

Si bien diversos autores han encontrado que la DTP de algunos suelos siguen distribuciones fractales, especialmente los finos (Bartoli et al. 1991; Tyler y Wheatcraft, 1992; Taguas et al. 1999; Filgueira et al. 2006a), en otros estudios se han identificado más de un modelo fractal en el mismo suelo (Wu et al. 1993; Bittelli et al. 1999). Tyler y Wheatcraft, (1992) concluyeron que sólo algunos suelos dentro del triángulo textural podrían tener este tipo de DTP. En este sentido, Filgueira et al. (2004) encontraron DTP en suelos del centro oeste de la provincia de Buenos Aires, que no se correspondían con este tipo de distribución.

Como ya mencionamos brevemente, para estudiar la DTP deben dispersarse las mismas, que se encuentran unidas en el suelo por la materia orgánica y otros posibles cementantes. Por ello, la DTP depende de los pretratamientos realizados sobre las muestras de suelos (Syvitski et al. 1991; Wu et al. 1993; Knösche et al. 1997; Konert y Vandenberghe, 1997; Beuselinck et al. 1998; Eshel et al. 2004; Dur et al. 2004; Filgueira et al. 2006 a, Filgueira et al. 2009, Gelati et al. 2010, Vdovic et al. 2010).

Stanchi et al. $(2006,2008)$ propusieron relacionar la dimensión fractal D con los procesos formadores, a partir de la evaluación de la DTP y la distribución de tamaño de microagregados (DTM) en varios órdenes de suelo, estudiando la sedimentación con diversos pretratamientos. En sus trabajos, evaluaron las distribuciones desde 1 hasta $2000 \mu \mathrm{m}$, mientras que otros autores (Bittelli et al. 1999; Filgueira et al. 2006a) utilizaron el modelo fractal sólo para describir las fracciones arena y arcilla-limo por separado. Behzad et al. (2015) recomiendan en su trabajo informar los puntos de corte inferior y superior de la escala fractal, es decir, acotar los tamaños de partículas para los que se ajustó el modelo y por otro lado que la determinación exacta de su dimensión fractal requiere una medición precisa y cuidadosa de la DTP. 
En muchos casos la DTP y DTM no muestran un estricto comportamiento fractal en términos de la bondad de ajuste a una ley potencial fractal, Stanchi et al. $(2006,2008)$ propusieron arbitrariamente que una DTP era fractal si el $\mathrm{R}^{2}$ resultante del ajuste de los datos experimentales a la función potencial que los describe, era mayor que 0,95 . Trabajos como éstos revelan que esta teoría, aplicada a los suelos está aún lejos de considerarse exacta y no queda claro, en ciertos casos, cuando la distribución de un conjunto de partículas puede ser considerada fractal, fuera de toda arbitrariedad.

El interés por el rol de la materia orgánica (MO) y el uso de la geometría Fractal en la Ciencia del Suelo está vigente y se desarrolla hacia la conexión biológica (Six et al. 2004; Caruso et al. 2011).

En nuestro cao lo que haremos Profundizar en el conocimiento de un tema

En el grupo de Física Aplicada de la Facultad de Ciencias Agrarias y Forestales de La Plata se ha trabajado desde hace años en investigación sobre DTP de suelos y las distintas metodologías para obtener las mismas (Filgueira et al. 2003, 2004, 2005, 2006a, 2006b, 2009; Gelati et al. 2008, 2010, 2016, 2018, 2019).

Hasta ahora no se ha investigado en nuestro país la evolución de la dimensión fractal de fragmentación $(D)$ con el grado de dispersión de una muestra, por lo que no se encuentran antecedentes bibliográficos. Esto implicaría estudiar la evolución de la DTM y la D con el contenido de MO. En otras palabras, para una DTP fractal, al ser la DTM un sistema heterogéneo (DTP+MO) se abre la posibilidad de investigar si la DTM continúa conformando una distribución fractal, o no.

Muchos suelos de pradera pampeana evolucionaron a partir del Loess. Uno de los mecanismos importantes de formación del suelo ha sido a través de la agregación de partículas primarias, mediante la $\mathrm{MO}$, para formar microagregados o pseudopartículas y finalmente, agregados. Esto es de nuestro interés, porque serían los primeros estadios de la formación de la estructura del suelo por acción de la MO. Los nuevos conocimientos que se adquieran en este tema serán de importancia en la Física de Suelos.

Se plantearon las siguientes hipótesis y objetivos:

\subsection{Hipótesis}

1.6.1 La distribución de partículas y microagregados de suelos, de clima templado de la pradera pampeana, independientemente de la clase textural y el contenido de materia orgánica, responde a una distribución tipo fractal. 
1.6.2 La dimensión fractal de fragmentación $\mathrm{D}$, es sensible al tamaño y cantidad de microagregados - partículas y, por ende, al contenido de materia orgánica del suelo.

1.6.3 Se puede obtener información sobre el estado de la microestructuración de un suelo a través del estudio de la distribución de partículas, microagregados y el análisis fractal de fragmentación

\subsection{Objetivos generales}

1.7.1 Investigar el comportamiento fractal de la distribución de tamaños de partículas (DTP) y de la distribución de tamaño de microagregados (DTM) del suelo.

1.7.2 Investigar el proceso de microagregación en suelos con texturas contrastantes, resultante de la unión de partículas elementales y la formación de microagregados por acción de la materia orgánica.

1.7.3 Asociar la distribución de tamaño de partículas con el material original y los procesos de transporte y sedimentación.

\subsection{Objetivos particulares}

1.8.1 Obtener por sedimentación la distribución de tamaño de partículas y microagregados de suelos pampeanos e interpretar las curvas de distribución resultantes, mediante la geometría fractal, obteniendo las dimensiones de fragmentación $\mathrm{D}$ y el parámetro $\mathrm{C}$.

1.8.2 Investigar si el modelo fractal es un indicador sensible para cuantificar el proceso de microagregación producido por la materia orgánica.

1.8.3 Estudiar al microscopio la presencia de partículas y microagregados en las diferentes fracciones granulométricas, principalmente el dominio arcilla y limo, para los diferentes pretratamientos.

1.8.4 Realizar un modelo fractal que interprete y explique el proceso la sedimentación diferencial del material original en la Pradera Pampeana. 
Materiales y métodos

Capítulo 2 


\section{Materiales y Métodos}

\subsection{Sitios de estudio y muestreo}

Se trabajó con muestras superficiales $(0-20 \mathrm{~cm})$ de suelos agrícolas de la Pampa Ondulada y Pampa Arenosa. Los sitios de muestreo georreferenciados fueron detallados en el anexo. En la Figura 2.1 se presenta la zona de trabajo que abarcó una amplitud de $5^{\circ} 22^{\prime} 41,1^{\prime \prime}$ que equivale aproximadamente a $500 \mathrm{~km}$ en el transecto este - oeste.

Las posiciones georreferencias obtenidas al momento del muestreo pueden encontrarse detalladas en Vinculos|Latitud y Longitud muestras.xlsx

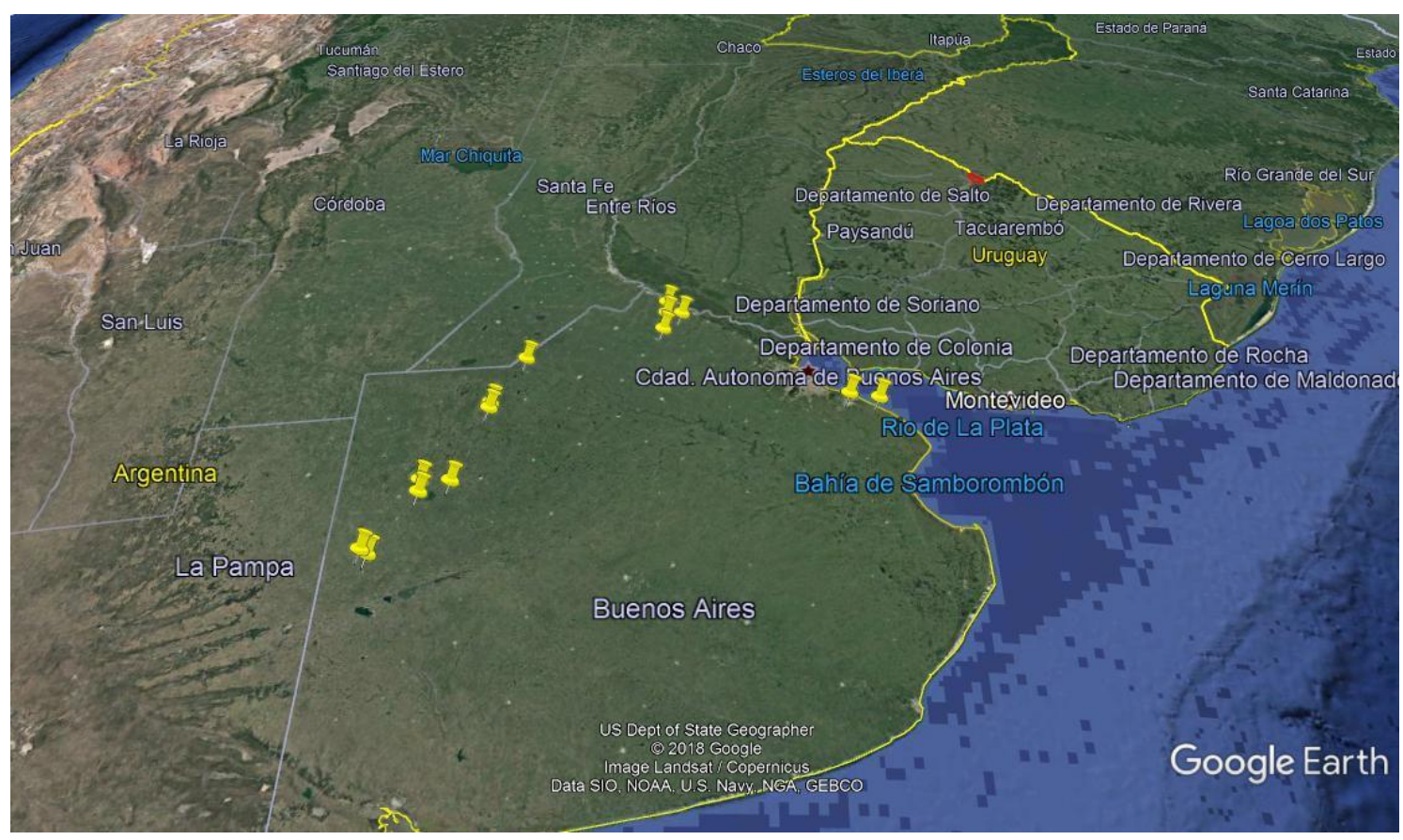

Figura 2.1. Mapa con los sitios de muestreo georreferenciados, cada punto representa las muestras del tratamiento bajo alambrado y agrícola

La amplitud espacial en el transecto buscó captar variabilidad en la distribución de tamaño de partículas (DTP) de las muestras en estudio. Las mismas correspondieron a dos situaciones para un mismo suelo "bajo alambrado" (BA) y "agrícola" (Agríc). Los BA se obtuvieron de ámbitos poco disturbados, mientras que el Agríc, correspondió con el lote trabajado. El muestreo se realizó de manera compuesta, con un mínimo de 10 submuestreos de barreno para cada muestra.

\subsection{Tratamiento de las muestras y determinaciones de laboratorio}

Muestra general, fueron secadas en estufa a $40^{\circ} \mathrm{C}$, con circulación forzada de aire, desagregadas, tamizadas por $2 \mathrm{~mm}$, etiquetadas y envasadas. Se realizaron determinaciones 
generales y específicas para los objetivos propuestos. Cuando fue necesario las mismas fueron acondicionadas de manera específica según los requisitos del protocolo.

\subsection{1 $p H$ real, $p H(1: 2,5)$}

Se evaluó el pH del suelo por vía potenciométrica, con una relación 1:2,5 de suelo: agua. Este parámetro es una medida elemental para caracterizarlo y refleja, en forma aproximada, la actividad de los iones hidrógenos $\left(\mathrm{H}^{+}\right)$de la solución del suelo (factor intensidad), los que tienen un efecto marcado sobre la fertilidad del mismo (disponibilidad de nutrimentos vegetales, SAMLA, 2004).

\subsubsection{Capacidad de Intercambio catiónico (CIC)}

La CIC es una medida de la cantidad de cargas negativas presentes en el suelo, debido principalmente a sustituciones isomórficas internas en las láminas de los minerales silicatados, disociación de radicales de ácidos orgánicos y adsorción diferencial de ciertos iones sobre la superficie de las partículas.

Se evaluó por el método del acetato de amonio $1 \mathrm{~N} \mathrm{pH} \mathrm{7.} \mathrm{La} \mathrm{evaluación} \mathrm{de} \mathrm{la} \mathrm{ClC}$ se

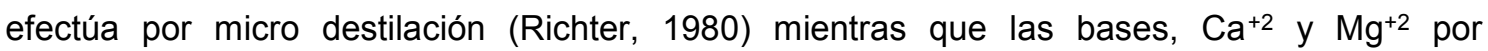
complejometría con EDTA; $\mathrm{Na}^{+}$y $\mathrm{K}^{+}$por fotometría de llama (SAMLA, 2004).

\subsubsection{Carbono orgánico total (COT)}

Es una de las caracterizaciones habituales de los suelos, pues interviene en los procesos físicos, físico químicos y químicos del suelo. Algunas de las propiedades físicas relacionadas con la materia orgánica son el color, la estructura, la adsorción, retención y permeabilidad del agua. Al mismo tiempo, la dinámica de la biomasa edáfica se ve afectada por el nivel y calidad del mencionado componente orgánico.

El método de COT por Walkley y Black, micrométodo (SAMLA, 2004) está basado en la recomendación de Métodos Analíticos del Instituto Nacional de Edafología y Agrobiología "José M. Albareda" (1973) de España, y de lo sugerido por The Laboratory Methods and Data Exchange Programme (1988) en sus procedimientos LABEX para análisis de suelos. El método se basa en el procedimiento propuesto por Walkley y Black (1934). El carbono es oxidado con una mezcla de $\mathrm{K}_{2} \mathrm{Cr}_{2} \mathrm{O}_{7}$ y $\mathrm{H}_{2} \mathrm{SO}_{4}$ concentrado y el exceso de dicromato se titula con reductor ferroso. Este método no oxida completamente a la materia orgánica sino a la fracción más lábil. Walkley y Black propusieron un factor de corrección de 1,3, basado en una oxidación promedio del $77 \%$, aceptado universalmente. Actualmente, se conoce que la oxidación varía desde 60 a $86 \%$. En nuestro país, Richter et al. (1975), determinaron, sobre suelos de la pradera pampeana, una oxidación promedio del $82 \%$, siendo entonces el factor de corrección 1,2, en lugar de 1,3, en esos suelos. Por lo tanto, se considera que este método da una aproximación semicuantitativa 
del carbono orgánico debido a la falta de un factor apropiado para cada suelo analizado. No obstante, en los análisis de rutina es ampliamente utilizado por sus ventajas de sencillez, rapidez y economía.

\subsubsection{Carbono orgánico particulado}

El carbono orgánico particulado (COP) se determinó siguiendo el procedimiento del libro Tecnologías en análisis de suelos, capítulo IV, modificado por Galantini (comunicación personal, 2014):

El COP está integrado por:

Carbono orgánico particulado grueso (COPG), es el CO de la fracción retenida en el tamiz de $105 \mu \mathrm{m}$ y ponderado por la relación entre la masa retenida en el tamiz de $105 \mu \mathrm{m}$ y la masa total.

Carbono orgánico particulado fino (COPF), es el CO de la fracción retenida en el tamiz de $53 \mu \mathrm{m}$ y ponderado por la relación entre la masa retenida en el tamiz de $53 \mu \mathrm{m}$ y la masa total.

Carbono orgánico asociado a la fracción mineral (COM): es el CO de la fracción $<53 \mu \mathrm{m}$ y ponderado por la relación entre la masa $<53 \mu \mathrm{m}$ y la masa total.

EI COT puede expresarse:

$$
C O T=C O P G+C O P F+C O M
$$

El COM puede obtenerse por diferencia.

Se pesó, anotando la masa, próxima a $50 \mathrm{~g}$ de suelo, se le agregaron 10 esferas de vidrio y $200 \mathrm{~mL}$ de agua. Las muestras se colocaron en un agitador rotativo por 12 a $18 \mathrm{~h}$, según la textura. Luego la suspensión fue volcada sobre dos tamices apilados, 105 y $53 \mu \mathrm{m}$.

Las muestras fueron lavadas con agua corriente y enjuagadas con agua destilada. EI material de cada tamiz fue extraído y secado a $105^{\circ} \mathrm{C}$, luego fue cuantificado en masa y se evaluó el CO por Walkley y Black micrométodo, sobre cada fracción retenida en los tamices.

Como ejemplo se muestra el cálculo para la fracción COPG:

$$
\operatorname{COPG}\left(\mathrm{g} \mathrm{kg}^{-1}\right)=\frac{M_{105}}{M_{T}} \times \% \mathrm{CO}_{(105)} \times 10 \quad \text { Ecuación 2-1 }
$$

COPG $\left(\mathrm{g} \mathrm{kg}^{-1}\right)$ = carbono orgánico de la fracción 2000-105 $\mu \mathrm{m}$

$\mathrm{M}_{105}(\mathrm{~g})=$ masa seca $\mathrm{a} 105^{\circ} \mathrm{C}$ retenida en el tamiz de $105 \mu \mathrm{m}$ 
$\mathrm{M}_{\mathrm{T}}(\mathrm{g})$ = masa total inicial

$\mathrm{CO}_{105}(\%)=$ porcentaje de carbono orgánico, evaluado sobre la fracción retenida en el tamiz de $105 \mu \mathrm{m}$.

10 es el factor para obtener el resultado en $\mathrm{g} \mathrm{kg}^{-1}$

\subsubsection{Fraccionamiento del COM}

Se procedió de similar manera al protocolo detallado en 2.2.4, realizando algunas modificaciones que se detallan a continuación.

Se pesaron, anotando la masa, próxima a $95 \mathrm{~g}$ y $70 \mathrm{~g}$ en suelos franco arenosos y francolimosos, respectivamente, se agregaron 10 esferas de vidrio y $250 \mathrm{~mL}$ de agua destilada. Las muestras se colocaron en un agitador rotativo por $18 \mathrm{~h}$. Luego la suspensión fue volcada sobre dos tamices apilados, 105 y $53 \mu \mathrm{m}$.

Las muestras fueron lavadas con agua destilada, se recolectó la suspensión resultante y la misma fue vertida y enrasada en probetas de $2000 \mathrm{~mL}$.

Las suspensiones fueron homogenizadas mediante un émbolo, a los tiempos correspondientes a los tamaños aparentes de 50, 20 y $2 \mu \mathrm{m}$, se realizaron extracciones de 500 $\mathrm{mL}$, se vertieron en vasos de precipitados, se llevaron a sequedad a $105^{\circ} \mathrm{C}$. Las masas fueron cuantificadas por gravimetría y el contenido de CO de cada subfracción por Walkley y Black micrométodo.

El aporte acumulado de CO que realiza cada subfracción, se calculó empleando la Ecuación 2.1, mientras que el aporte de cada una, se obtuvo por diferencia.

\subsubsection{Sedimentaciones}

Se realizaron en probetas de $1000 \mathrm{~mL}$ con agua destilada y dos pretratamientos:

(a) Sin materia orgánica (SMO). Cómo indica el pretratamiento para pipeta de Robinson (Gee y Bauder, 1986)

(b) Con materia orgánica (CMO). La muestra de suelo se humectó con $15 \mathrm{~mL}$ de alcohol etílico, se dejó en reposo 5 minutos, se enrasó e inmediatamente fue sedimentada.

\subsubsection{S Sin Materia Orgánica (SMO)}

Se realizó siguiendo el protocolo del método de la pipeta de Robinson (Gee y Bauder, 1986). Se lo consideró como el pretratamiento de máxima dispersión 
Se eliminó la $\mathrm{MO}$ con peróxido de hidrógeno $\left(\mathrm{H}_{2} \mathrm{O}_{2}\right)$ y calor. Luego la muestra fue secada a $105^{\circ} \mathrm{C}$ y pesada.

Sobre la muestra seca y SMO, se agregó $\mathrm{NaOH}, 1 \mathrm{~N}$ como dispersante químico a razón de $5 \mathrm{~mL}$ por muestra, se dejó en reposo 12 a 16 h y luego se realizó la dispersión mecánica en agitador rotativo con esferitas de vidrio durante 12 a 18 h, según la textura del suelo.

Probetas preparadas con la suspensión dispersa y enrasadas con agua destilada fueron agitadas con un émbolo y se realizaron las extracciones a los tiempos establecidos. Las arenas fueron tamizadas y cuantificadas por gravimetría luego de la sedimentación.

\subsubsection{Con Materia Orgánica (CMO). Pretratamiento con materia orgánica}

Se pesó la masa de suelo seco, con materia orgánica, se le agregó $15 \mathrm{~mL}$ de alcohol etílico para eliminar el aire de los poros sin que se produzca el estallido de los agregados y así mantener la estructura, similar a la técnica de estabilidad estructural de Le Bissonnais (1996), se dejó por 5 minutos hasta humectación total de la muestra.

Se agregó agua destilada hasta enrasar en $1000 \mathrm{~mL}$, inmediatamente se agitó 20 veces y se realizaron las extracciones a los tiempos previstos.

Los cálculos de las masas acumuladas en porciento se realizaron como indica la Ecuación 2.2.

$$
\text { masa acumulada }(\%)<\varnothing=\frac{M_{S} \text { Vprobeta }}{M_{T} \text { Vpipeta }} 100 \quad \text { Ecuación 2-2 }
$$

Donde:

Ms (g): masa seca obtenida para el diámetro $(100,50,25,10,5$ y $2 \mu \mathrm{m})$

$\mathrm{M}_{\mathrm{T}}(\mathrm{g})$ : masa total inicial, pesada al iniciar el ensayo

$V_{\text {probeta: }}$ volumen de la probeta, $1000 \mathrm{~mL}$

$V_{\text {pipeta: }}$ volumen de la pipeta de aforo simple, $25 \mathrm{~mL}$ 
Para calcular el tiempo de extracción de un diámetro aparente $\emptyset_{\mathrm{i}}$, se empleó la ecuación de Stokes, que contempla la viscosidad de la suspensión, profundidad de extracción de la muestra y la densidad real de partículas, considerada $2,65 \mathrm{~g} \mathrm{~cm}^{-3}$.

$$
\Delta t=\frac{18 \times \Delta y \times \eta}{g \times \emptyset^{2} \times\left(\delta_{c}-\delta_{f}\right)} \quad \text { Ecuación 2-3 }
$$

Donde:

$\Delta t=$ tiempo en segundos $(\mathrm{s})$

$\Delta y=$ profundidad de extracción de la muestra mediante una pipeta $(\mathrm{cm})$

$\eta=$ coeficiente de viscosidad $\left(\operatorname{din~} \mathrm{sm}^{-2}\right)$

$\mathrm{g}=$ aceleración de la gravedad $\left(\mathrm{cm} \mathrm{s}^{-2}\right)$

$\varnothing=$ diámetro aparente $(\mathrm{cm})$

$\delta \mathrm{c}=$ densidad real de partículas $\left(2,65 \mathrm{~g} \mathrm{~cm}^{-3}\right)$

$\delta \mathrm{f}=$ densidad del fluido $\left(1,00 \mathrm{~g} \mathrm{~cm}^{-3}\right)$

La clasificación de los tamaños de partículas se empleó el criterio de USDA, mencionado en la Introducción general.

Puesto que la viscosidad es función de la temperatura, en el laboratorio se midió la "temperatura de la suspensión" como insumo para estimar su viscosidad en cada medición.

En la Tabla 2.1, se presentan los tiempos calculados con la ecuación de Stokes para los diámetros aparentes evaluados en función de la profundidad de extracción y a $20^{\circ} \mathrm{C}$ :

Tabla 2.1 Esquema de tiempos necesarios para obtener las fracciones acumuladas de diámetro aparente $\varnothing$ $(\mu m)$ en función de la profundidad de extracción $(\Delta y)$ y la temperatura de la suspensión.

\begin{tabular}{cccccc} 
Hora & Minutos & Segundos & $\Delta \mathrm{y}(\mathrm{cm})$ & $\mathrm{t}\left({ }^{\circ} \mathrm{C}\right)$ & $\varnothing(\mu \mathrm{m})$ \\
\hline- & - & 22 & 20 & 20 & 100 \\
- & 1 & 29 & 20 & 20 & 50 \\
- & 5 & 58 & 20 & 20 & 25 \\
- & 18 & 38 & 10 & 20 & 10 \\
1 & 14 & 30 & 10 & 20 & 5 \\
3 & 52 & 49 & 5 & 20 & 2
\end{tabular}




\subsubsection{Textura aparente}

Se la definió como los porcentajes de las fracciones de los tamaños arena limo y arcilla, cuando la muestra sedimentó en las condiciones del pretratamiento CMO.

Arena aparente (A ap): fracción comprendida entre $50<\varnothing(\mu \mathrm{m})<2000$. El porcentaje se obtuvo por diferencia como se indica:

$$
\operatorname{Aap}(\%)=100-(L+\operatorname{arc})_{a p} \quad \text { Ecuación 2-4 }
$$

Limo aparente $(L$ ap): fracción comprendida entre $2<\varnothing(\mu \mathrm{m})<50$. El porcentaje se obtuvo como se indica:

$$
L a p(\%)=(L+\operatorname{arc})_{a p}-\operatorname{arc}_{a p} \quad \text { Ecuación 2-5 }
$$

Arcilla aparente (arc ap): fracción en porcentaje $\varnothing(\mu \mathrm{m})<2 \mu \mathrm{m}$.

\subsubsection{Fracciones sin materia orgánica y con materia orgánica, comparación directa}

Se compararon los valores en porcentajes de cada fracción real y su par aparente, esto se realizó a nivel grupal e individual. Se compararon los valores en porcentaje de arenas reales - arenas aparentes, limos reales - limos aparentes y arcillas reales - arcillas aparentes.

\subsubsection{Relaciones entre las fracciones sin materia orgánica y con materia orgánica}

Se realizó el cociente entre el porcentaje real de partículas (SMO) y el porcentaje aparente (CMO). Esta relación permitió cuantificar el efecto microestructurante de la MO.

$$
\begin{aligned}
& \text { Relación Arenas }=\frac{\text { Arenas }(\%)}{\text { Arenas aparentes (\%) }} \quad \text { Ecuación 2-6 } \\
& \text { Relación Limos }=\frac{\text { Limos (\%) }}{\text { Limos aparentes (\%) }} \quad \text { Ecuación 2-7 } \\
& \text { Relación arcillas }=\frac{\text { arcillas }(\%)}{\text { arcillas aparentes (\%) }} \quad \text { Ecuación 2-8 }
\end{aligned}
$$

\subsubsection{Delta de las fracciones granulométricas}

Con esta expresión se buscó relacionar la diferencia entre fracciones y el contenido real de la misma, a modo de comparar por unidad de la fracción analizada independizando del contenido real inicial. Se calculó según la Ecuación 2.9, los resultados se expresaron en porcentaje. El signo positivo indica que la fracción incrementó su contenido, mientras que el signo negativo indica que la fracción disminuyó por efecto de la microestructuración. 


$$
\Delta F(\%)=\frac{F(C M O)-F(S M O)}{F(S M 0)} 100 \quad \text { Ecuación 2-9 }
$$

Donde:

$\Delta \mathrm{F}(\%)$, es la variación de la fracción analizada según los pretratamientos

$\mathrm{F}(\mathrm{CMO})=$ porcentaje de la fracción obtenida en las condiciones $\mathrm{CMO}$

$\mathrm{F}(\mathrm{SMO})=$ porcentaje de la misma fracción obtenida en las condiciones SMO.

\subsubsection{Modelo fractal aplicado a la distribución de tamaño de partículas}

El modelo fractal basado en el análisis de masa propuesto por (Tyler y Wheatcraft, 1992) citado en la Ecuación 1.5 es:

$$
\frac{M_{\left(r<R_{\text {Lsup }}\right)}}{M_{T}}=\left(\frac{r}{R_{\text {Lsup }}}\right)^{3-D}=C r^{3-D}
$$

Donde:

Mт: es la masa total de la distribución de las partículas

$M_{(r<R L s u p)}$ es la masa parcial hasta el radio $r$ y menor que $R_{L \text { sup. }}$

$r$ : es el radio mayor de la masa parcial acumulada y menor que $R_{\text {Lsup. }}$

$\mathrm{R}_{\mathrm{Lsup}}$ el radio límite superior de tamaño para el comportamiento fractal

C: es una constante

D: es la dimensión fractal de fragmentación.

Expresando la Ecuación 1.5 en función de los diámetros $(\varnothing)$ y modificando la nomenclatura de la fracción de masa, se obtiene la siguiente expresión:

$$
\frac{M_{p}}{M_{T}}=\left(\frac{\emptyset_{p}}{\emptyset_{M}}\right)^{3-D} \therefore M_{p}=\frac{M_{T}}{\left(\emptyset_{M}\right)^{3-D}}\left(\emptyset_{p}\right)^{3-D}=C \emptyset_{p}^{3-D} \quad \text { Ecuación 2-10 }
$$

Donde:

La nomenclatura es similar a la anterior, coincidiendo $\mathrm{M}_{\mathrm{T}}$, C y D, mientras que, $\mathrm{M}_{\mathrm{p}}$ : es la masa parcial acumulada hasta el tamaño $\emptyset_{p}(\mu \mathrm{m})$ y $\emptyset_{M}(\mu \mathrm{m})$ es el diámetro máximo hasta donde alcanza un comportamiento fractal DTP o DTM. 
La regresión potencial obtenida con los datos experimentales es del tipo:

$$
m(\%)=a \emptyset^{b} \quad \text { Ecuación 2-11 }
$$

Comparando esta Ecuación 2.11 con el modelo teórico, Ecuación. 2.10, se deduce que "a" es igual a "C" y "b" es igual a "3-D".

Linealizando mediante logaritmos la Ecuación 2.11

$$
\log m(\%)=\log a+b \log \emptyset \quad \text { Ecuación } 2-12
$$

De esta recta, con la ordenada al origen se estima la "C", de la Ecuación 2.10 y de su pendiente, "b", la dimensión fractal " $D$ ".

$$
\begin{gathered}
C=10^{\log a} \\
D=3-b
\end{gathered}
$$

\subsubsection{Escala sedimentológica. Parámetro Phi y calificaciones}

A los resultados de las sedimentaciones, se les realizó el análisis empleando la metodología propuesta por McManus (1988).

Las curvas de distribución de tamaño de partículas (DTP) y distribución de tamaño de microagregados (DTM) de masa acumulada fueron representados en función de Phi definido como: "el logaritmo negativo en base dos del diámetro de partículas expresado en mm", como indica la expresión:

$$
\text { Phi }=-\log _{2} d(\mathrm{~mm}) \quad \text { Ecuación 2-13 }
$$

El parámetro Phi, representa la curva acumulada, similar a como ocurre durante la sedimentación: primero las arenas, luego los limos y por último las arcillas.

Las equivalencias entre los diámetros expresados en mm y Phi se presentan en la Tabla 2.2. 
Tabla 2.2 Equivalencia de diámetro ( $\varnothing)$ en milímetros y el parámetro Phi

\begin{tabular}{cc}
$\varnothing(\mathrm{mm})$ & $\mathrm{Phi}=-\mathrm{Log}_{2} \varnothing$ \\
\hline 2,000 & $-1,00$ \\
1,000 & 0,00 \\
0,750 & 0,42 \\
0,500 & 1,00 \\
0,250 & 2,00 \\
0,125 & 3,00 \\
0,100 & 3,32 \\
0,075 & 3,74 \\
0,050 & 4,32 \\
0,020 & 5,64 \\
0,010 & 6,64 \\
0,005 & 7,64 \\
0,002 & 8,97
\end{tabular}

Con esta metodología de análisis, pueden estimarse parámetros estadísticos como la media y el desvío estándar entre otros, los cuales permiten clasificar el material sedimentado según la escala propuesta en la Tabla 2.3. La media de la distribución califica la granulometría, mientras que el desvío estándar la calidad de selección.

Tabla 2.3. Calificación de las sedimentaciones a partir de los valores de la media, expresados en Phi y desvío estándar

\begin{tabular}{clcl} 
Media & Calificación & Desvío estándar & \multicolumn{1}{c}{ Calificación } \\
\hline 0,500 & arena gruesa & 0,00 & muy bien seleccionada \\
1,000 & arena gruesa & 0,35 & bien seleccionada \\
1,001 & arena mediana & 0,50 & moderadamente bien seleccionada \\
2,000 & arena mediana & 1,00 & pobremente seleccionada \\
2,001 & arena fina & 2,00 & muy pobremente seleccionada \\
3,000 & arena fina & 4,00 & extremadamente mal seleccionada \\
3,001 & arena muy fina & 5,00 & extremadamente mal seleccionada \\
4,000 & arena muy fina & & \\
4,001 & limo grueso & & \\
5,000 & limo grueso & & \\
5,001 & limo mediano & & \\
6,000 & limo mediano & & \\
6,001 & limo fino & & \\
8,000 & limo fino & & \\
8,001 & arcilla & & \\
10,000 & arcilla & &
\end{tabular}




\subsubsection{Mineralogía de arcillas}

Se realizó por análisis de difracción de rayos $X(D R X)$ de la roca total y de la fracción arcilla para caracterizar la composición mineralógica del material, así como para identificar los minerales de arcilla (argilominerales) y su abundancia relativa.

Las muestras se desagregaron y pulverizaron en un mortero de ágata siguiendo los métodos clásicos. Los minerales de arcilla se obtivieron a partir del pipeteo de la fracción $<2 \mu \mathrm{m}$ en una suspensión con agua destilada siguiendo la Ley de Stokes. Se prepararon 3 muestras según el método de los portaobjetos de vidrio:

a) natural, muestra secada al aire en el laboratorio a temperatura ambiente.

b) glicolada, muestra expuesta a los vapores de una solución de etilenglicol por lo menos durante $24 \mathrm{~h}$.

c) calcinada, muestra llevada a $550^{\circ} \mathrm{C}$ durante $2 \mathrm{~h}$.

Se utilizó un sistema de difractometría de rayos X marca PANalytical, modelo X'Pert PRO con lámpara de $\mathrm{Cu}(\mathrm{k \alpha}=1.5403 \AA$ ) que opera a $40 \mathrm{~mA}$ y $40 \mathrm{kV}$ en el Centro de Investigaciones Geológicas (La Plata). Se escanearonn ángulos $2 \theta$ de 4 a $37^{\circ}$ para las muestras de roca total, 2 a $32^{\circ}$ para las muestras naturales de la fracción arcilla, 2 a $27^{\circ}$ para las muestras glicoladas de esa misma fracción, y 3 a $15^{\circ}$ para las muestras calcinadas, con una velocidad de escaneo de $0,04 \%$ s.

La composición mineral de cada muestra se determinó según su abundancia relativa (Poiré, 1987): muy abundante (>50\%), abundante (26-50\%), moderada (16-25\%), escasa (6$15 \%)$, muy escasa (1-5\%), y trazas $(<1 \%)$.

Las escalas calificativas aplicadas a los resultados a nivel cualitativo y cuantitativo propuesta por Poire (comunicación personal) se presentan en la Tabla 2.4: 
Tabla 2.4. Calificación cuali cuantitiva de los resultados de las determinaciones mineralógicas por difracción de rayos $X$. (Comunicación personal Poire)

Q: cuarzo
Feld K: feldespato potásico
PI: plagioclasa
Arc: arcilla
Ca: calcita
D: dolomita
S: siderita

Q: cuarzo

A: analcima

Cli: clinoptilolita

L: laumontita

Y: yeso

An: anhidrita

Op: ópalo

$\mathrm{Hm}$ : hematita

Py: pirita
Sm: esmectita

CE: capas

expansivas

C: clorita

IS: illita-esmectita

CS: clorita-esmectita

I: illita

K: caolinita

Cr: cristalinidad

MB: muy buena

B: buena

R: regular

M: mala

NC: no

cuantificable

EA: extremadamente abundante ( $>80 \%$ )

MA: muy abundante (entre 50 y $80 \%$ )

A: abundante (entre 30 y $50 \%$ )

Mo: moderado (entre 15 y $30 \%$ )

$\mathrm{E}$ : escaso (entre 5 y15\%)

ME: muy escaso (entre 1 y 5\%)

$\mathrm{T}$ : trazas $(<1 \%)$

\subsubsection{Masa seca a $105^{\circ} \mathrm{C}$}

Todas las determinaciones se expresaron sobre suelo seco a $105^{\circ} \mathrm{C}$, empleando el factor de humedad, cuando fue necesario:

$$
M_{S}=\frac{M_{h}}{(1+C H)} \quad \text { Ecuación 2-14 }
$$

Donde:

$$
\begin{aligned}
& M_{s}(g)=\text { masa seca } a 5^{\circ} \mathrm{C} \\
& M_{h}(g)=\text { masa húmeda } \\
& (1+\mathrm{CH})=\text { Factor de humedad }
\end{aligned}
$$

\subsubsection{Fotografías al microscopio}

Se utilizó un equipo Gemalux XSZ-H, las fotos se tomaron con una cámara Motic 1000 y se empleó un software Motic Image Plus 2.0. 
Una alícuota de las sedimentaciones se colocaron sobre un portaobjeto y se armaron preparados, en algunos casos se empleó safranina para dar color, otras veces Lugol. Luego se les colocó un cubre objeto y se sellaron los bordes con esmalte transparente. Las fotografías se tomaron de sedimentaciones en tres suelos con ambos pretratamientos, Se emplearon aumentos de 40, 100250 y 400x. Las escalas gráficas se agregaron a las fotos para facilitar su interpretación.

\subsubsection{Análisis estadístico}

A los resultados experimentales se les aplicó el modelo de análisis de la varianza y cuando se detectaron diferencias significativas las medias se separaron mediante un test de Tuckey $(: 0,05)$.

Los análisis de regresión se realizaron por el método de mínimos cuadrados. La formación de grupos homogéneos se realizó por separación de clúster de k medias.

Se emplearon los coeficientes de correlación para evaluar el grado de asociación entre resultados del modelo fractal con datos experimentales.

Los análisis se realizaron empleando el software estadístico Infostat 2018, versión libre (Di Rienzo et al. 2018). 
Resultados generales

Capítulo 3 


\section{Resultados generales}

En este capítulo, se presentan y analizan aquellos resultados obtenidos en la caracterización general de las muestras, haciendo posible la asociación con series de suelo descriptas. Con los resultados de las sedimentaciones sin materia orgánica y con materia orgánica se calcularon las relaciones entre las fracciones reales (partículas) y aparentes (microagregados) y su relación con el carbono orgánico asociado a la fracción mineral. Se muestran y comparan las imágenes obtenidas al microscopio.

\subsection{Características de los suelos estudiados}

\subsubsection{Asociación de los sitios estudiados a series de suelos}

Empleando las coordenadas geográficas junto a los resultados de las determinaciones de $\mathrm{pH}$ real, capacidad de intercambio catiónico, carbono orgánico total, textura, empleando Geolnta, (2018) y cartas de suelo, los suelos muestreados se asociaron a las siguientes series descriptas. En la Tabla 3.1 se encuentran las referencias entre número de suelo, condición en la que se obtuvo, bajo alambrado o agrícola, serie asociada y la clasificación taxonómica. 
Tabla 3.1. Número de suelo, condición del sitio muestreado (BA) bajo alambrado, (Agríc) agrícola, la serie de suelo con la que fue asociado y la clasificación taxonómica (USDA, Soil Taxonomy). (*)Tapto es un localismo empleado en Argentina, no reconocido por USDA, Soil Taxonomy.

\begin{tabular}{|c|c|c|c|}
\hline Suelo $N^{\circ}$ & Condición & Serie (símbolo) & Taxonomía \\
\hline 1 & BA & Arrecifes (Ar) & Argiudol Acuico, fina, illítica, térmica \\
\hline 2 & Agric & & \\
\hline 3 & BA & Portela (Po) & Argiudol Vértico, fina, illítica, térmica \\
\hline 4 & Agric & & \\
\hline 5 & BA & Ramallo (Ra) & Argiudol Vértico, fina, illítica, térmica \\
\hline 6 & Agric & & \\
\hline 7 & BA & Urquiza (Ur) & Paleudol Típico,fina, illítica, térmica \\
\hline 8 & Agric & & \\
\hline 9 & BA & Norumbega (No) & Hapludol Éntico, franca gruesa; mixta, térmica \\
\hline 10 & Agric & & \\
\hline 11 & BA & Piedritas (Pas) & Hapludol Éntico, franca gruesa, mixta, térmica \\
\hline 12 & Agric & & \\
\hline 13 & BA & Saforcada (Sf) & Hapludol Éntico, franca gruesa; mixta, térmica \\
\hline 14 & Agric & & \\
\hline 16 & BA & Bombeador (Bo) & Argiudol típico, arcillosa fina illítica térmica. \\
\hline 17 & Agric & & \\
\hline 18 & BA & Centeno $(\mathrm{Cn})$ & Argiudol típico, arcillosa fina illítica térmica. \\
\hline 19 & Agric & & \\
\hline 20 & BA & Magdalena (Md) & Paleudol Vértico, muy fina, illítica, térmica \\
\hline 21 & Agric & & \\
\hline 24 & BA & Bolivar (Bv) & Hapludol Entico, franca gruesa, mixta, térmica \\
\hline 25 & Agric & & \\
\hline 26 & BA & Ortiz de Rosas (Or) & $\begin{array}{l}\text { Hapludol Tapto Árgico, franca fina, mixta, } \\
\text { térmica }\left({ }^{*}\right)\end{array}$ \\
\hline 27 & Agric & & \\
\hline 28 & BA & Saboya (Sy) & Argiudol Típico, franca fina, mixta, térmica \\
\hline 29 & Agric & & \\
\hline 30 & Agric & Pirovano (Pv) & $\begin{array}{l}\text { Udipsamente Tapto Mólico, franco gruesa, } \\
\text { mixta, térmica }\left(^{*}\right)\end{array}$ \\
\hline 31 & Agric & Piedritas (Pas) & Hapludol Éntico, franca gruesa, mixta, térmica \\
\hline
\end{tabular}

\subsection{Formación de grupos}

Los suelos fueron separados en grupos, empleando el método estadístico de análisis de conglomerados de $\mathrm{K}$ medias, utilizando como variables, los porcentajes de arena, arcilla y el diámetro correspondiente a la media de la distribución de masa en función de Phi. En la Tabla 3.2 se presentan los valores medios obtenidos de las variables para cada grupo. 
Tabla 3.2 Valores medios de los porcentajes de arena (A\%), arcilla (arc\%) y diámetro correspondiente a la media (Phi), expresado en $\mu m$. Grupos 1, 2 y 3

\begin{tabular}{cccc} 
Variable & Grupo 1 & Grupo 2 & Grupo 3 \\
\hline A (\%) & 53,9 & 80,6 & 8,7 \\
arc (\%) & 13,9 & 6,7 & 25,1 \\
Phi medio & 48 & 164 & 18
\end{tabular}

Con esta metodología, se formaron tres grupos principales:

Grupo 1 (G1), suelos francos y franco arenosos de la Pampa Arenosa.

Grupo 2 (G2), un suelo de clase textural areno franco de la Pampa Arenosa.

Grupo 3 (G3), suelos franco-limosos y franco arcillo limosos de la Pampa Ondulada.

En la Tabla 3.3 se presenta la distribución de los suelos en cada grupo principal, la condición de las muestras, bajo alambrado o agrícola y la serie taxonómica. 
Tabla 3.3. Detalle de los grupos, suelos que lo conforman, condición de muestreo y serie taxonómica asociada. $\left({ }^{*}\right)$ Tapto es un localismo empleado en Argentina, no reconocido por USDA, Soil Taxonomy.

\begin{tabular}{|c|c|c|c|c|c|}
\hline Grupo & Subgrupo & Suelo $N^{\circ}$ & Condición & Serie & Taxonomía \\
\hline \multirow{13}{*}{ G1 } & G1FA & 9 & $\mathrm{BA}$ & No & Hapludol Éntico, franca gruesa; mixta, térmica \\
\hline & G1FA & 10 & Agric & & \\
\hline & G1FA & 11 & BA & Pas & Hapludol Éntico, franca gruesa, mixta, térmica \\
\hline & G1FA & 12 & Agric & & \\
\hline & G1FA & 13 & BA & Sf & Hapludol Éntico, franca gruesa; mixta, térmica \\
\hline & G1FA & 14 & Agric & & \\
\hline & G1FA & 31 & Agric & Pas & Hapludol Éntico, franca gruesa, mixta, térmica \\
\hline & G1F & 24 & BA & $\mathrm{Bv}$ & Hapludol Éntico, franca gruesa, mixta, térmica \\
\hline & G1F & 25 & Agric & & \\
\hline & G1F & 26 & BA & Or & Hapludol Tapto Árgico, franca fina, mixta, térmica \\
\hline & G1F & 27 & Agric & & \\
\hline & G1F & 28 & $\mathrm{BA}$ & Sy & Argiudol Típico, franca fina, mixta, térmica \\
\hline & G1F & 29 & Agric & & \\
\hline G2 & & 30 & Agric & $\mathrm{Pv}$ & $\begin{array}{l}\text { Udipsamente Tapto Mólico }\left(^{*}\right) \text {, franco gruesa, mixta, } \\
\text { térmica }\end{array}$ \\
\hline \multirow[t]{14}{*}{ G3 } & & 1 & $\mathrm{BA}$ & $\operatorname{Ar}$ & Argiudol Acuico, fina, illíica, térmica \\
\hline & & 2 & Agric & & \\
\hline & & 3 & BA & Po & Argiudol Vértico, fina, illítica, térmica \\
\hline & & 4 & Agric & & \\
\hline & & 5 & BA & $\mathrm{Ra}$ & Argiudol Vértico, fina, illítica, térmica \\
\hline & & 6 & Agric & & \\
\hline & & 7 & BA & Ur & Paleudol Típico, fina, illítica, térmica \\
\hline & & 8 & Agric & & \\
\hline & & 16 & BA & Bo & Argiudol típico, arcillosa fina illítica térmica. \\
\hline & & 17 & Agric & & \\
\hline & & 18 & BA & $\mathrm{Cn}$ & Argiudol típico, arcillosa fina illítica térmica. \\
\hline & & 19 & Agric & & \\
\hline & & 20 & BA & Md & Paleudol Vértico, muy fina, illítica, térmica \\
\hline & & 21 & Agric & & \\
\hline
\end{tabular}

\subsection{Características de los grupos}

\subsubsection{Grupo 1}

Se formó con 13 suelos de la pampa arenosa. Las clases texturales de los suelos variaron entre franca y franca arenosa, siendo la fracción dominante la arena 53,9\%, le siguió el limo $32,2 \%$ y por último la arcilla con $13,9 \%$. Estos valores caracterizaron al grupo como franco arenoso.

En la escala sedimentológica, la media de $\mathrm{Phi}=4,39$ equivalente a $49,8 \mu \mathrm{m}$ y un desvío estándar de 2,04, lo clasificó como "limo grueso, muy pobremente seleccionado". (Figura 3.1). 
El contenido medio de COT resultó de $15,5 \mathrm{~g} \mathrm{~kg}^{-1}$ con un desvío estándar de $5,2 \mathrm{~g} \mathrm{~kg}^{-1}$, mientras que el rango de las muestras varió entre 9,6 y $27,4 \mathrm{~g} \mathrm{~kg}^{-1}$. El COPG fue de $2,8 \mathrm{~g} \mathrm{~kg}^{-1}$, el COPF de $2,0 \mathrm{~g} \mathrm{~kg}^{-1}$ y el COM de $10,8 \mathrm{~g} \mathrm{~kg}^{-1}$.

El análisis mineralógico, de roca total caracterizó a los suelos por una composición muy abundante de cuarzo (65\%), escasos feldespatos potásicos $(10 \%)$, abundante a moderado contenido de plagioclasa (10-20\%) y muy escaso contenido de argilominerales (1-5\%).

La composición proporcional de la fracción de argilominerales, fue muy abundante para la illita $(70 \%)$ y moderado contenido del complejo interestratificación de illita esmectita (30\%). En el Anexo pueden consultarse los espectros de las determinaciones realizadas.

Al sedimentar las muestras con el pretratamiento $\mathrm{CMO}$, se obtuvieron porcentajes medios de las fracciones aparentes de arena, limo y arcilla de $81,1 \% ; 18,0 \%$ y $0,9 \%$ respectivamente, lo que identificó al grupo con una clase textural aparente "arenoso franco". En la escala sedimentológica la media fue Phi $=2,55$ equivalente a $175,3 \mu \mathrm{m}$, y un desvío estándar de 2,17 $\mu \mathrm{m}$ y caracterizó al material como "arena fina, muy pobremente seleccionada".

\subsubsection{Grupo 2}

Este grupo se formó por el suelo 30 asociado a la serie Pirovano; un Udipsamente Tapto Mólico, franco gruesa, mixta, térmica. De textura areno franco. La fracción arena es dominante con $80,6 \%$, le sigue el limo, $12,7 \%$ y la menos representada es la arcilla, con $6,7 \%$, siendo la clase textural arenoso franco.

La DTP del grupo en la escala sedimentológica obtuvo una media de Phi=1,95 equivalente a $259,2 \mu \mathrm{m}$ y fue clasificado como "arena mediana, pobremente seleccionada". (Figura 3.1)

El contenido de COT fue de $9,8 \mathrm{~g} \mathrm{~kg}^{-1}$, equivalente $19,6 \mathrm{~g} \mathrm{~kg}^{-1}$ de MO. El COPG fue de $2,8 \mathrm{~g} \mathrm{~kg}^{-1}$, COPF de $0,9 \mathrm{~g} \mathrm{~kg}^{-1}$ y el COM fue de $6,3 \mathrm{~g} \mathrm{~kg}^{-1}$.

Al sedimentar el suelo CMO, se obtuvo $93,1 \%$ de pseudo arenas, 6,6\% de pseudo limos y $0,3 \%$ de pseudo arcillas, otorgándole al suelo una clase textural aparente "arenoso", en la escala sedimentológica se lo calificó como "arena mediana, pobremente seleccionada" con una media de $P h i=1,58$ equivalente a $333,3 \mu \mathrm{m}$ y 1,72 el desvío estándar.

\subsubsection{Grupo 3}

Se formó con suelos Argiudoles y Paleoudoles, de la Pampa Ondulada; suelos profundos evolucionados a nivel de Familia, fina - muy fina, illítica y de régimen térmico. La fracción dominante fue el limo con $66,7 \%$, le siguió la arcilla con $25,0 \%$ y por último la arena con $8,3 \%$. Con una media de $\mathrm{Phi}=5,78(18,2 \mu \mathrm{m})$ y un desvío estándar de 1,82 fue calificado como "limo 
mediano, pobremente seleccionado". (Figura 3.1). El contenido medio de COT resultó de 26,6 g $\mathrm{kg}^{-1}$ con un desvío estándar de $11,1 \mathrm{~g} \mathrm{~kg}^{-1}$, mientras que el rango de las muestras varió entre 15,9 y $54,9 \mathrm{~g} \mathrm{~kg}^{-1}$. El COPG fue de $2,6 \mathrm{~g} \mathrm{~kg}^{-1}$, COPF de $2,7 \mathrm{~g} \mathrm{~kg}^{-1}$ y COM fue de $21,3 \mathrm{~g} \mathrm{~kg}^{-1}$.

En el análisis de mineralogía, la roca total caracterizó a los suelos por una composición muy abundante de cuarzo (65\%), escasos feldespatos potásicos (10\%), abundante a moderado contenido de plagioclasa (20-40\%) y escaso contenido de argilominerales $(10 \%)$.

La composición proporcional de la fracción de argilominerales, fue muy abundante para la illita $(60 \%)$ y abundante la interestratificación de illita esmectita (40\%).

Como característica se destaca, el suelo Portela (Argiudol Vértico) que presentó moderado contenido de esmectitas $(30 \%)$, moderado contenido del complejo interestratificado illita esmectita (20\%) y abundante contenido de illita (50\%). En el Anexo pueden consultarse los espectros de las determinaciones realizadas.

Al sedimentar los suelos, $\mathrm{CMO}$, se encontró un corrimiento hacia clases texturales aparentes más gruesas, similar a los grupos anteriores. La distribución por fracciones resultó, arena $58,3 \%$, limo $39,2 \%$ y arcilla $2,5 \%$. La media de $P h i=3,58$ equivalente a $86 \mu \mathrm{m}$ y el desvío estándar de 2,74, lo calificó en la escala sedimentológica como "arena muy fina muy pobremente seleccionada".

\subsection{Relación entre el carbono orgánico asociado a la fracción mineral y el carbono} orgánico total

Se realizó una regresión entre los valores de COM en función del COT, empleando todos los suelos estudiados. El resultado obtenido, una regresión lineal significativa $\left(R^{2}=0,95\right.$. $p<0,0001)$. La ordenada al origen no obtuvo significancia $(p=0,51)$. Figura 3.2 .

$$
\begin{gathered}
\text { COM }=0,733 \text { COT }+0,586 \\
\mathrm{R}^{2}=0,95 . \mathrm{p}<0,0001 \\
9,62<\operatorname{COT}\left(\mathrm{g} \mathrm{kg}^{-1}\right)<54,87
\end{gathered}
$$

\subsection{Carbono orgánico asociado a la fracción mineral, en función del contenido de arcilla y arcilla más limo para los tres grupos}

Se realizaron regresiones, entre el COM en función del porcentaje de arcilla, separando las condiciones de "bajo alambrado" y "agrícola". Los suelos "bajo alambrado", mostraron elevada variabilidad, obteniendo $\left(R^{2}=0,29 . p=0,056\right)$. Los suelos agrícolas en cambio, obtuvieron mejor ajuste $\left(R^{2}=0,61\right.$. $\left.p<0,001\right)$ con el modelo lineal. Figura 3.3a 
Del mismo modo, se realizaron regresiones entre valores del COM en función del valor de arcilla + limo, obteniéndose para los suelos "bajo alambrado" una función lineal y significativa $\left(R^{2}=0,51 . p=0,006\right)$. Los suelos "agrícola" nuevamente obtuvieron mejor ajuste que los anteriores, $\left(R^{2}=0,65 . p<0,001\right)$. Las pendientes resultaron estadísticamente diferentes. Figura 3.3b.

El efecto de la agricultura sobre los suelos, redujo la cantidad de carbono orgánico total y por ende del carbono orgánico asociado a la fracción mineral.

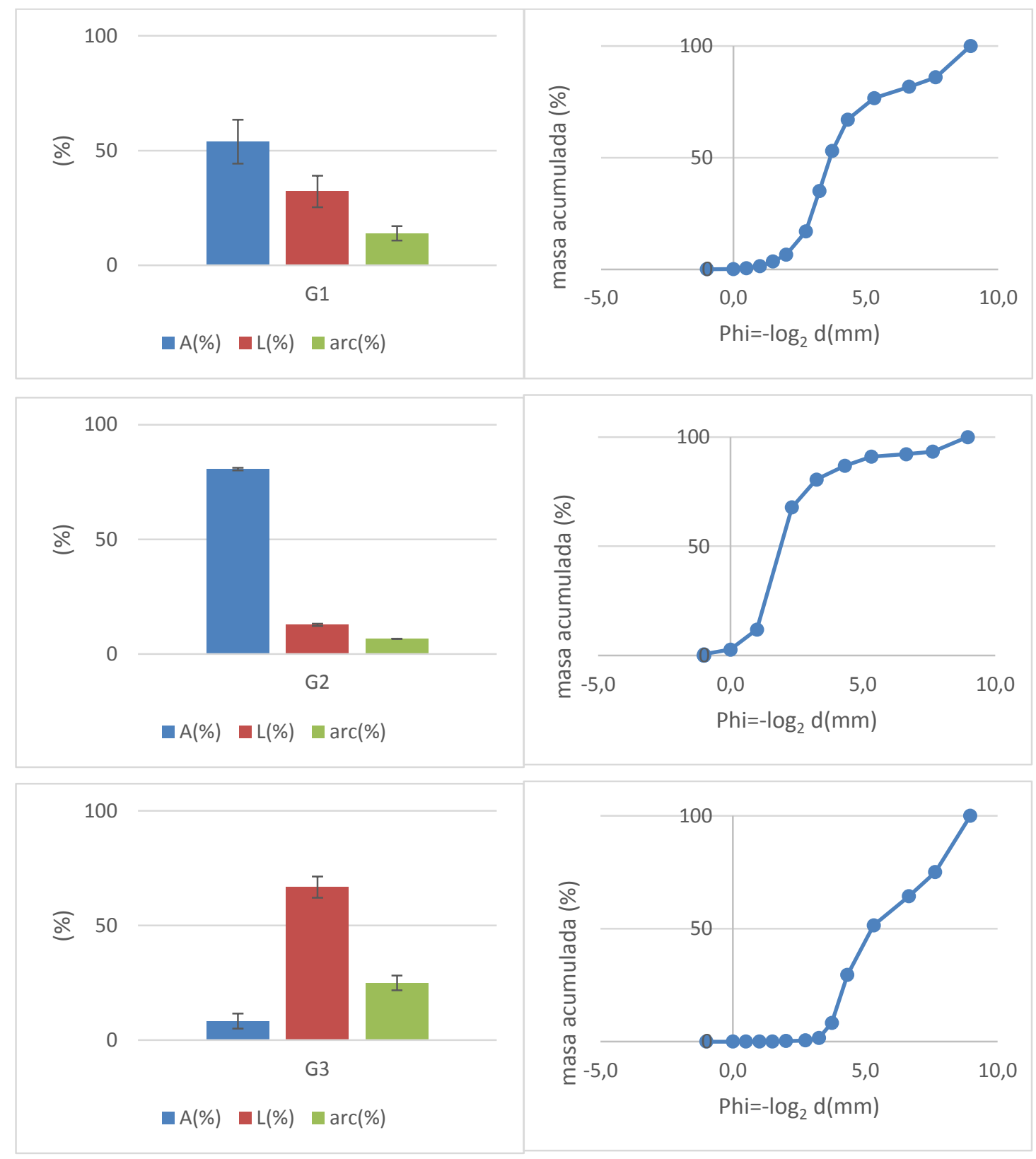

Figura 3.1. Proporciones medias y error estándar de arena limo y arcilla por grupo. Distribución de tamaño de partículas de masa acumulada en función de Phi. 


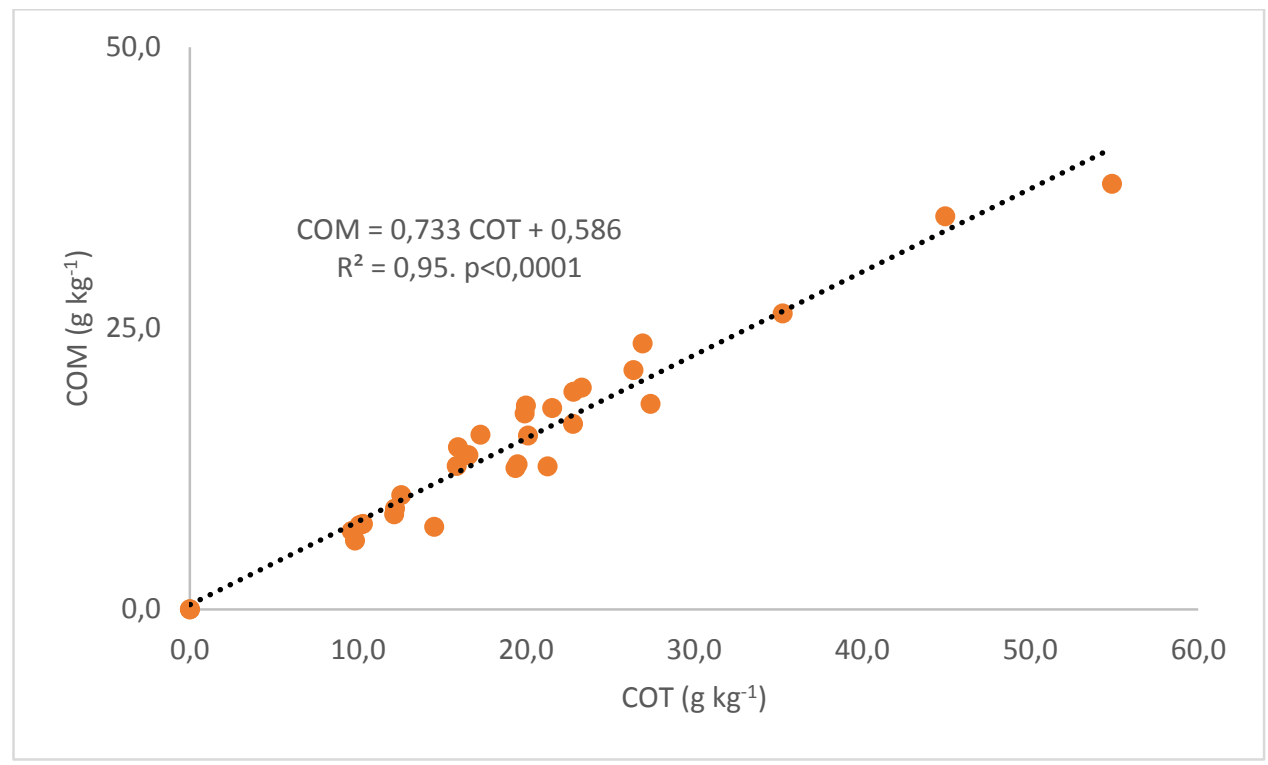

Figura 3.2. Relación del carbono orgánico particulado asociado a la fracción mineral (COM) en función del carbono orgánico total (COT), valores experimentales y recta de regresión.

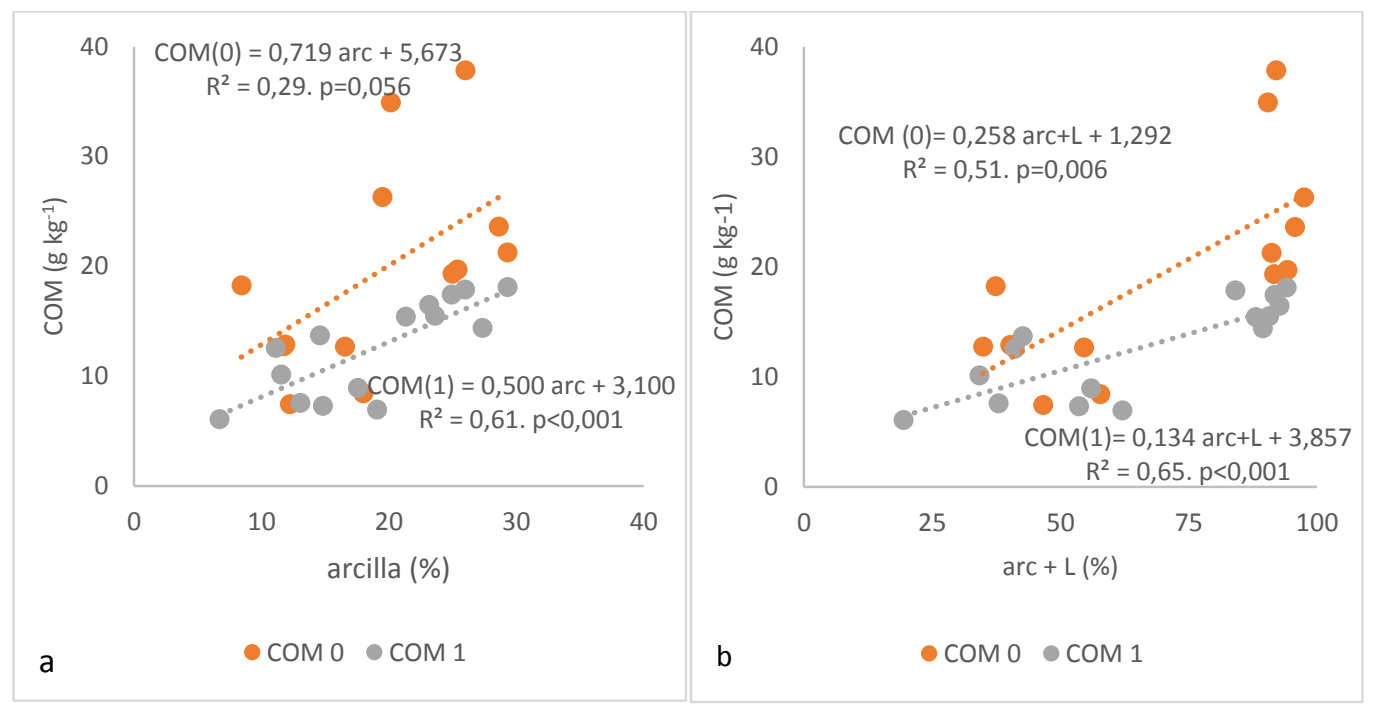

Figura 3.3. Carbono orgánico particulado asociado a la fracción mineral (COM), separados por tratamientos "bajo alambrado = 0 " y "agrícola = 1" y las correspondientes rectas de regresión, $a$, en función del contenido de arcillas y $b$ en función del contenido de arcilla + limo. 


\subsection{Fracciones granulométricas reales y aparentes}

Con los resultados de las sedimentaciones sin materia orgánica (SMO) y con materia orgánica ( $\mathrm{CMO}$ ) para los tamaños arena limo y arcilla en este apartado, se estudiaron las proporciones entre partículas y microagregados y las relaciones entre los microagregados y el contenido de COM. Los resultados expuestos representan los valores medios de cada grupo. Los valores individuales para cada suelo pueden consultarse dentro del archivo Excel VinculosITabla completa.xlsx (columnas AG-AL).

\subsubsection{Grupo 1}

En este grupo, de suelos de la Pampa Arenosa, los contenidos de COT $(p=0,58)$ y las fracciones de carbono orgánico particulado grueso (COPG - $p=0,53$ ), carbono orgánico particulado fino (COPF - $p=0,84)$ y carbono orgánico asociado a la fracción mineral (COM $p=0,68$ ), no se diferenciaron entre los tratamientos "bajo alambrado" y "agrícola" (Figura 3.4).

El análisis mediante la escala sedimentológica caracterizó al grupo SMO como "limo grueso muy pobremente seleccionado, media Phi = 4,39 (50 $\mu \mathrm{m})$. La sedimentación CMO calificó al grupo como "arena fina muy pobremente seleccionada", media Phi= 2,55 (175 $\mu \mathrm{m})$.

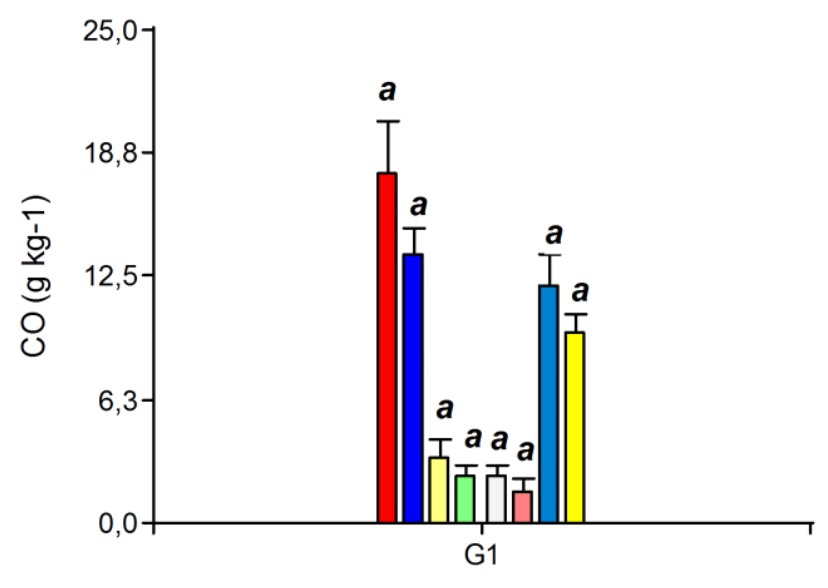

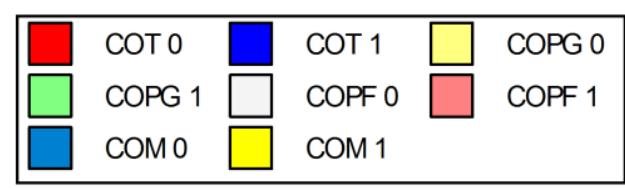

Figura 3.4. Carbono orgánico total (COT) y carbono orgánico particulado, grueso (COPG), fino (COPF) y asociado a la fracción mineral (COM). Muestras bajo alambrado (0) y muestras agrícola (1). Las comparaciones son de a pares $0-1$, medias con una letra común no son significativamente diferentes $(p>0,05)$. 


\subsubsection{Tamaño arena}

Los valores de las arenas reales (SMO) 53,9\% y arenas aparentes (CMO) $81,1 \%$ se diferenciaron estadísticamente $(p<0,0001)$. El promedio del cociente A/Aap resultó igual a 0,7 (Figura 3.5).

La variación relativa (Ecuación 2.9), " $\Delta A$ ", tuvo una media de $54,2 \%$, un $C V$ de $45,0 \%$, dentro del rango de 24,7 a $104,5 \%$.

El Grupo pasó de una clase textural franco arenosa a una clase textural aparente areno franco.

Se analizó la cantidad de arenas aparentes para los tratamientos "bajo alambrado" y "agrícola", no se encontró diferencias $(p=0,96)$.

\subsubsection{Tamaño limo}

Los contenidos de limos reales (SMO) $32,2 \%$ y limos aparentes (CMO) $18,1 \%$ se diferenciaron estadísticamente $(p<0,0001)$. El promedio del cociente L/Lap resultó de 1,8 por efectos de la microestructuración. La variación relativa, estimada con la Ecuación 2.9 " $\Delta L$ ", obtuvo una media de $-43,2 \%$, un CV del $24,2 \%$ dentro de un rango (-60,3 y $-25,9 \%$ ). (Figura 3.5 )

Se analizó la cantidad de limos aparentes para los tratamientos "bajo alambrado" y "agrícola", no encontrándose diferencias $(p=0,93)$.

\subsubsection{Tamaño arcilla}

Los valores de arcilla real (SMO) 13,9\% y arcilla aparente (CMO) 0,9\% se diferenciaron estadísticamente $(p<0,0001)$. El promedio del cociente arc/arcap resultó de 18,7 , por efectos de

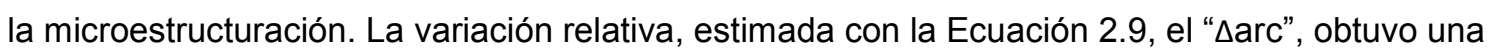
media de $-94,0 \%$, un CV de 2,2\%, dentro del rango de -96,9 a -89,2\%. Esta fracción resultó ser la menos dispersa de las tres. (Figura 3.5)

Se analizó la cantidad de arcillas aparentes para los tratamientos "bajo alambrado" y "agrícola", no encontrándose diferencias $(p=0,70)$. 

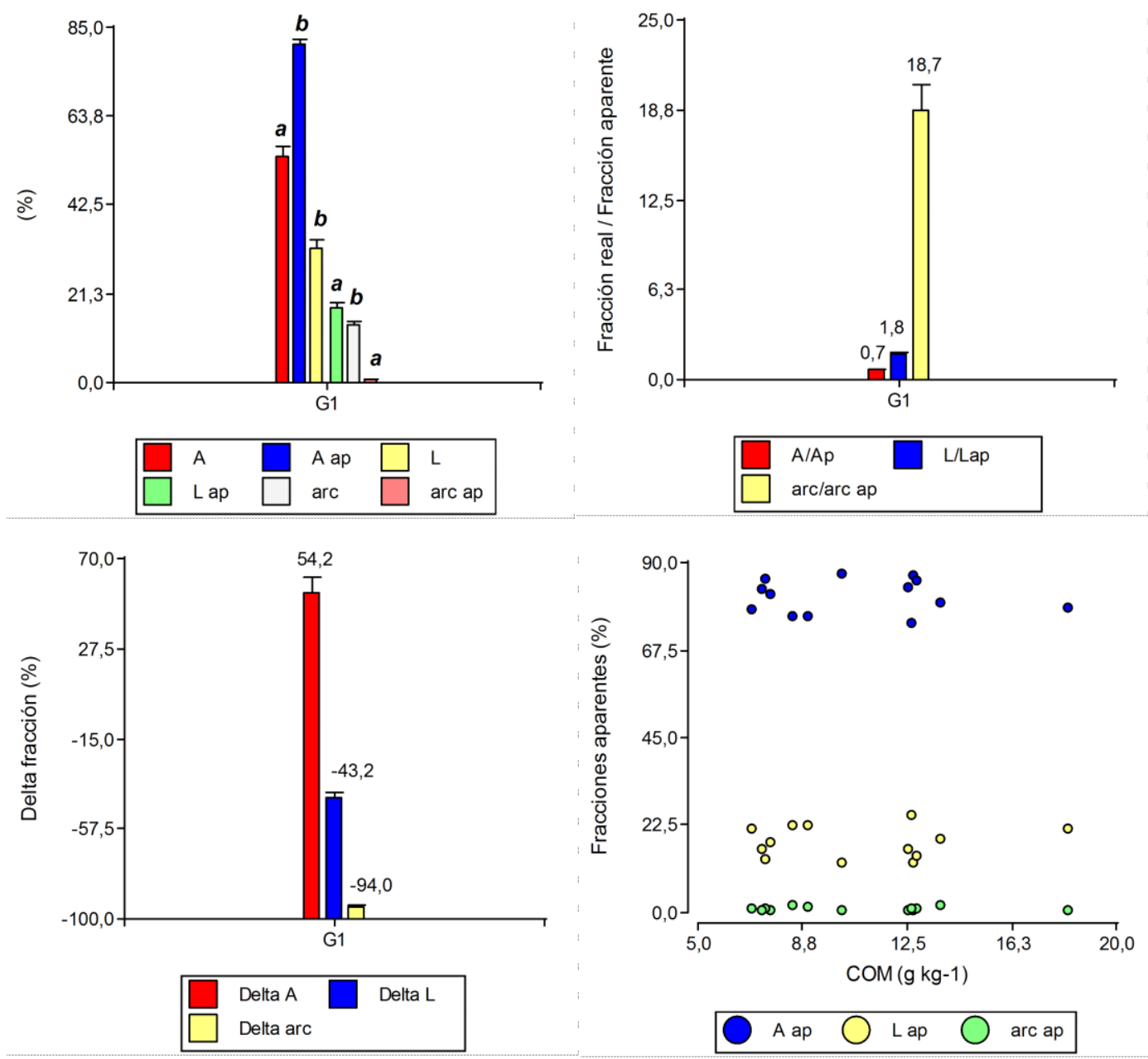

Figura 3.5. Fracciones texturales para el G1 según pretratamientos SMO y CMO. Relaciones entre fracción real y aparente. Variación relativa, Delta de cada fracción. Fracciones aparentes de arena, limo y arcilla en función del COM. Medias para la misma fracción, con una letra común no son significativamente diferentes $(p>0,05)$.

\subsubsection{Grupo 2}

La naturaleza de este suelo de textura areno franco, es diferente a los integrantes de los Grupos 1 y 3, se estudió por separado. Se consideró importante mantener este Grupo de manera diferenciada, no como excepción sino como el representante de los suelos más gruesos de la Pradera Pampeana, siendo valiosa su participación en la modelización fractal propuesta para los suelos Pampeanos en el Capítulo 6.

\subsubsection{Tamaño arena}

Las arenas reales (SMO) $80,6 \%$ y las arenas aparentes (CMO) 93,1\% mostraron similar comportamiento al Grupo 1. El cociente $A / A a p$ resultó igual a 0,9 . La variación relativa $\Delta A$ tuvo un valor de 15,6\%. (Figura 3.6) 


\subsubsection{Tamaño limo}

El porcentaje de limos reales (SMO) 12,7\% respecto a los limos aparentes (CMO) 6,5\%, mostraron similar tendencia que el Grupo 1, la relación L/Lap fue 1,9. La variación relativa, estimada por el $\Delta \mathrm{L}$, obtuvo una media de $-48,6 \%$. (Figura 3.6)

\subsubsection{Tamaño arcilla}

El porcentaje de arcillas reales (SMO) 6,7\% respecto de las arcillas aparentes (CMO) 0,34\%, mostró similar tendencia que el Grupo 1, la relación entre arc/arcap fue 19,8. La variación relativa, estimada por el $\Delta$ arc, obtuvo una media de $-95,0 \%$.
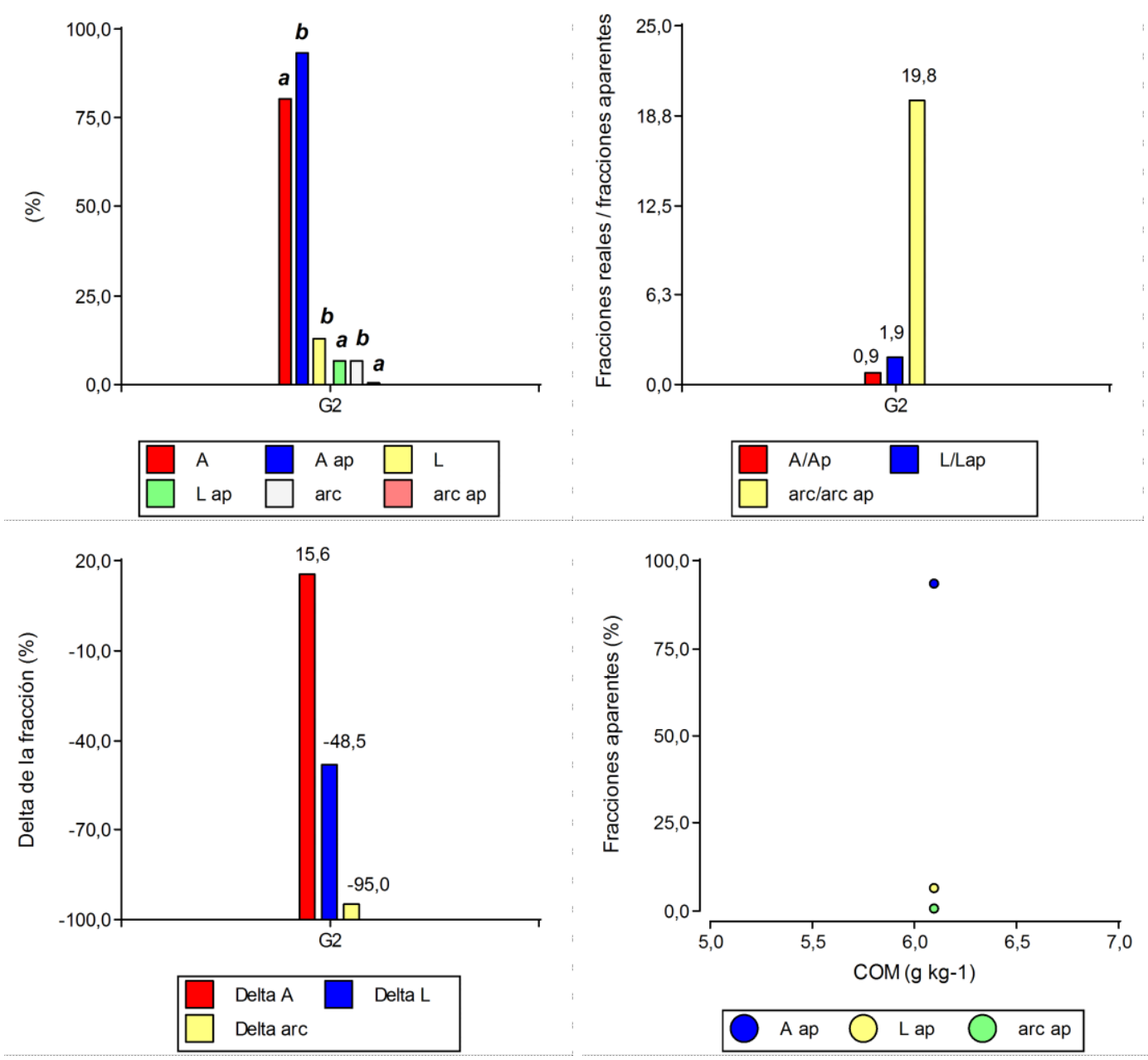

Figura 3.6. Fracciones texturales para el G2 según pretratamientos $S M O$ y CMO. Relaciones entre fracción real y aparente. Variación relativa, Delta de cada fracción. Fracciones aparentes de arena, limo y arcilla en función del COM. 


\subsubsection{Grupo 3}

En este Grupo se encontraron diferencias entre los tratamientos "bajo alambrado y agrícola" en el contenido de carbono orgánico total, (COT - $p=0,01)$ y en las fracciones carbono orgánico particulado fino (COPF - $p=0,02$ ) y carbono orgánico asociado a la fracción mineral $(\mathrm{COM}-\mathrm{p}=0,005)$. No mostrando diferencias la fracción de carbono orgánico particulado grueso (COPG - $p=0,15)$ (Figura 3.7).

Recordemos la clasificación obtenida en la escala sedimentológica para el grupo SMO de "limo mediano pobremente seleccionado" media Phi= 5,78 $(18,3 \mu \mathrm{m})$ mientras que cuando sedimentó CMO pasó a "arena muy fina, muy pobremente seleccionada" con una media de Phi= $3,58(82,6 \mu \mathrm{m})$.

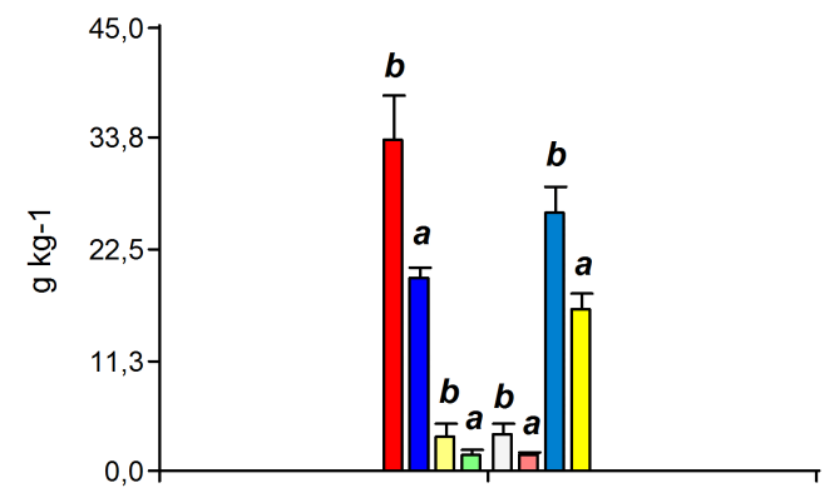

G3

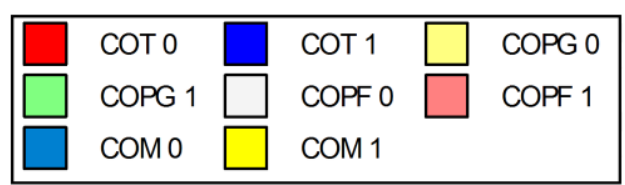

Figura 3.7. Carbono orgánico total (COT) y carbono orgánico particulado (COP) de la fracción gruesa (COPG), COP de la fracción fina (COPF) y carbono orgánico asociado a la fracción mineral (COM) separadas por "O" bajo alambrado y " 1 " agrícola. Las comparaciones son de a pares $0-1$, medias con una letra común no son significativamente diferentes ( $p>0,05)$.

\subsubsection{Tamaño arena}

Los valores de las arenas reales (SMO) $8,3 \%$ y arenas aparentes (CMO) $58,3 \%$ se diferenciaron estadísticamente $(p<0,0001)$. El promedio del cociente A/Aap resultó igual a 0,1. La variación relativa, $\Delta \mathrm{A}$, tuvo una media de $780,4 \%$, un $C V$ de $74,5 \%$, dentro del rango de 252,7 $-2.489,1 \%$.

Puesto que se encontraron diferencias en los contenidos de carbono orgánico para las condiciones bajo alambrado y agrícola, se analizó la cantidad de arenas aparentes entre estas 
condiciones. Se encontraron diferencias significativas $(p<0,0001)$ siendo el valor de $63,0 \%$ para el bajo alambrado y 53,7\% para el agrícola. Un análisis de regresión entre los valores de arena aparente en función del COM, Figura 3.8, evidenció una relación no lineal positiva $\left(R^{2}=0,53\right.$ y $p<0,0001)$.

$$
\begin{gathered}
A_{a p}(\%)=25,344 \times \operatorname{COM}^{0,275} \\
R^{2}=0,53 . p<0,0001 \\
14,41<\operatorname{COM}\left(\mathrm{g} \mathrm{kg}^{-1}\right)<37,86
\end{gathered}
$$

\subsubsection{Tamaño limo}

Los contenidos de limos reales (SMO) 66,7 \% y limos aparentes (CMO) 39,2 \% se diferenciaron estadísticamente $(p<0,0001)$. El promedio del cociente $L / L a p$ resultó igual a 1,7. La variación relativa, estimada por el $\Delta \mathrm{L}$, obtuvo una media de $-40,9 \%$ y un $\mathrm{CV}$ del $24,8 \%$ dentro de un rango $(-56,0$ y $-22,7 \%)$.

Se analizó la cantidad de limos aparentes para las condiciones BA y Agríc, se encontraron diferencias significativas $(p<0,001)$, siendo el valor de $34,9 \%$ para bajo alambrado y 43,5\% para Agríc. Un análisis de regresión entre los valores de limo aparente en función del COM, (Figura 3.8) evidenció una relación no lineal negativa $\left(R^{2}=0,57\right.$ y $\left.p<0,0001\right)$.

$$
\begin{gathered}
L_{a p}(\%)=114,19 \times \operatorname{COM}^{-0,358} \\
\mathrm{R}^{2}=0,57 . \mathrm{p}<0,0001 \\
14,41<\operatorname{COM}\left(\mathrm{g} \mathrm{kg}^{-1}\right)<37,86
\end{gathered}
$$

\subsubsection{Tamaño arcilla}

Los valores de arcilla real (SMO) 25,0\% y arcilla aparente (CMO) 2,5\% se diferenciaron estadísticamente $(p<0,0001)$. El promedio del cociente arc/arcap resultó igual a 11,0. La variación relativa, calculada a través del $\Delta$ arc, obtuvo una media de $-90,0 \%$, un CV de $3,7 \%$, dentro del rango de $-94,4$ a $-81,6 \%$. 
Se analizó la cantidad de arcillas aparentes para los tratamientos BA y Agríc. Se encontraron diferencias significativas ( $p=0,034)$, siendo los valores de arcilla aparente $2,1 \%$ para BA y 2,9\% para Agríc. Se realizó un análisis de la relación entre los valores de arcilla aparente y el COM, obteniéndose una función lineal significativa $\left(R^{2}=0,28 p=0,053\right)$. Figura 3.8.

$$
\begin{gathered}
\operatorname{arc}_{a p}=-0,054 \times \operatorname{COM}+3,622 \\
R^{2}=0,28 \cdot p=0,053 \\
14,41<\operatorname{COM}\left(g^{~ k g-1}\right)<37,86
\end{gathered}
$$
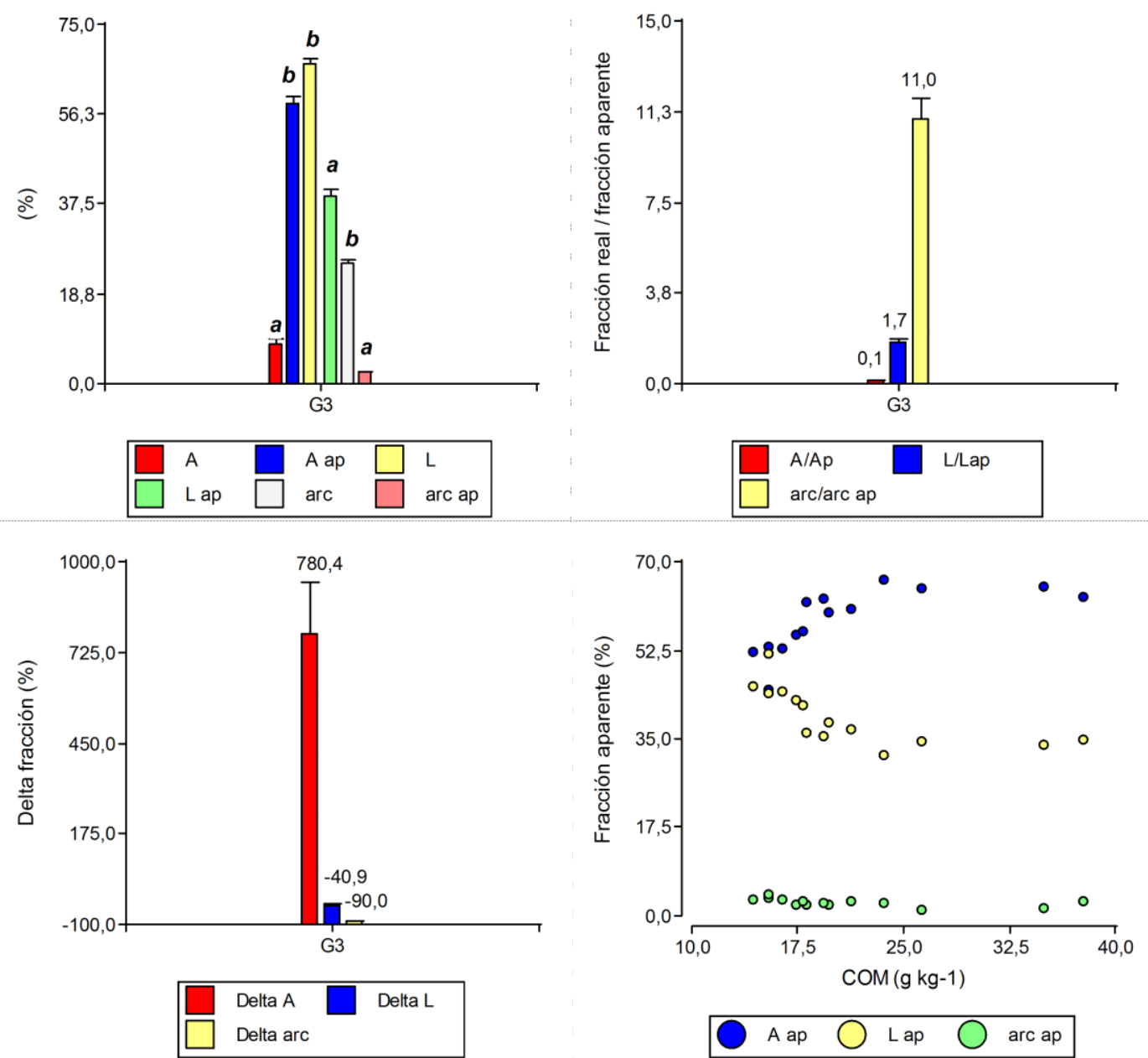

Figura 3.8. Fracciones texturales para el G3 según pretratamientos SMO y CMO. Relaciones entre fracción real y aparente. Variación relativa, Delta de cada fracción. Fracciones aparentes de arena, limo y arcilla en función del COM. Medias, para la misma fracción, con una letra común no son significativamente diferentes $(p>0,05)$ 


\subsection{Estudio del carbono orgánico particulado y fraccionamiento del carbono orgánico asociado a la fracción mineral}

Durante el desarrollo de la tesis se observó la variabilidad en los contenidos de COT y COP que presentaban las muestras, de los diferentes grupos y condiciones de muestreo. Se consideró que merecía un análisis más detallado y se profundizó la investigación realizando el fraccionamiento del carbono orgánico asociado a la fracción mineral, se trabajó con microagregados de 50, 20 y $2 \mu \mathrm{m}$, en condiciones de uso y textura contrastante. Los suelos estudiados, se detallan en la Tabla 3.4 .

El fraccionamiento del COM, en los tamaños 50,20 y $2 \mu \mathrm{m}$, se realizó siguiendo el protocolo detallado en el punto 2.2 .5 de materiales y métodos.

Tabla 3.4. Suelos empleados realizar el fraccionamiento del COM, Serie Pas: Piedritas, Bo: Bombeador, C.T clase textural FA: franco arenoso, FL: franco limoso. Condición del suelo BA: bajo alambrado, Agríc: agrícola

\begin{tabular}{cccc} 
Suelo & Serie & C.T. & Cond \\
\hline S11 & Pas & FA & BA \\
S12 & Pas & FA & Agric \\
S16 & Bo & FL & BA \\
S17 & Bo & FL & Agric
\end{tabular}

Los suelos bajo alambrado obtuvieron valores absolutos de carbono orgánico total (COT) y carbono orgánico particulado grueso (COPG), carbono orgánico particulado fino (COPF) y carbono orgánico asociado a la fracción mineral (COM) mayores que los correspondientes a los pares agrícola. Se observa que el S11, superó en COT y COP al S17, evidenciando la potencialidad de acumular $\mathrm{CO}$ de este suelo franco arenoso y por otro lado la pérdida de $\mathrm{CO}$ del S17, por la actividad agrícola. Figura 3.9a

Al representar las fracciones del COP, en porcentaje respecto al COT, Figura 3.9b, se observa que proporcionalmente el COM (\%) es mayor en los suelos agrícolas, por un enriquecimiento residual del COM, debido a la pérdida de las fracciones más reactivas del CO.

Del fraccionamiento del COM resultó que la cantidad de $\mathrm{CO}$ es mayor en los microagregados de menor tamaño. (Tabla 3.5).

Los suelos BA presentaron valores absolutos de $\mathrm{CO}$ acumulado mayores que los pares agrícolas. (Figura 3.10a), invirtiéndose la tendencia cuando el CO acumulado se expresó en forma relativa al COT (Figura 3.10b). El comportamiento de las fracciones del COM, fue similar al de las fracciones de COP (Figura 3.9 a y b). 
La subdivisión del COT y los aportes absolutos $\left(\mathrm{g} \mathrm{kg}^{-1}\right)$, que realiza cada fracción estudiada en este apartado se observan en la Figura 3.11a, mientras que el aporte relativo (\%), se puede observar en la Figura 3.11b.

Los suelos BA, contienen mayor cantidad de COT y el mismo se encuentra más distribuido en las diferentes clases de tamaño, siendo notorio en el suelo más fino, debido a las mayores posibilidades de arreglo de los complejos arcillo húmicos. Los suelos "agrícola", mostraron menor cantidad de COT y menor distribución del mismo, concentrándolo en las clases más finas siendo notorio el S17, donde el CO aportado por la clase 2-20 $\mu \mathrm{m}$ alcanza al $57 \%$ del COT. El efecto de protección de los complejos arcillo húmicos puede ser una de las razones y la pérdida de las fracciones más reactivas del $\mathrm{CO}$, presente en los microagregados mayores, reduzca la resistencia de los mismos y no resistan al proceso de dispersión, propio de la metodología, acumulándose en los tamaños inferiores.

Para obtener una medida comparativa rápida de las proporciones de $\mathrm{CO}$ entre suelos bajo alambrado y agrícola, se realizó el cociente entre los $\mathrm{CO}$ aportados por cada una de las fracciones estudiadas. En el suelo FA, la máxima variación se observó en el COPF, siendo el BA 4,2 veces mayor que el correspondiente al suelo agrícola. En el caso del suelo $\mathrm{FL}$, la máxima variación se observó en el CO de la fracción 20 - $50 \mu \mathrm{m}$, siendo el BA 6,7 veces mayor que el correspondiente al suelo agrícola. Figura 3.12.

Tabla 3.5. Valores de carbono orgánico (CO) medido sobre las diferentes fracciones de microagregados. EI CO acumulado es lo porción CO que aporta al carbono orgánico total (COT)

\begin{tabular}{ccccccccc} 
& \multicolumn{2}{c}{$\mathrm{S} 11$} & \multicolumn{2}{c}{$\mathrm{S} 12$} & \multicolumn{2}{c}{$\mathrm{S} 16$} & \multicolumn{2}{c}{$\mathrm{S} 17$} \\
Diam & $\mathrm{CO}$ & $\mathrm{CO}_{\text {Acum }}$ & $\mathrm{CO}$ & $\mathrm{CO}_{\text {Acum }}$ & $\mathrm{CO}$ & $\mathrm{CO}_{\text {Acum }}$ & $\mathrm{CO}$ & $\mathrm{CO}_{\text {Acum }}$ \\
$(\mu \mathrm{m})$ & & & & \multicolumn{2}{c}{$\left(\mathrm{g} \mathrm{kg}^{-1}\right)$} & & & \\
\hline 2 & 74,40 & 4,60 & 58,50 & 4,66 & 55,90 & 3,64 & 30,30 & 3,56 \\
20 & 70,40 & 13,91 & 48,00 & 10,90 & 43,70 & 17,08 & 28,40 & 14,21 \\
50 & 59,50 & 17,14 & 41,40 & 12,99 & 39,50 & 27,94 & 22,30 & 15,52
\end{tabular}

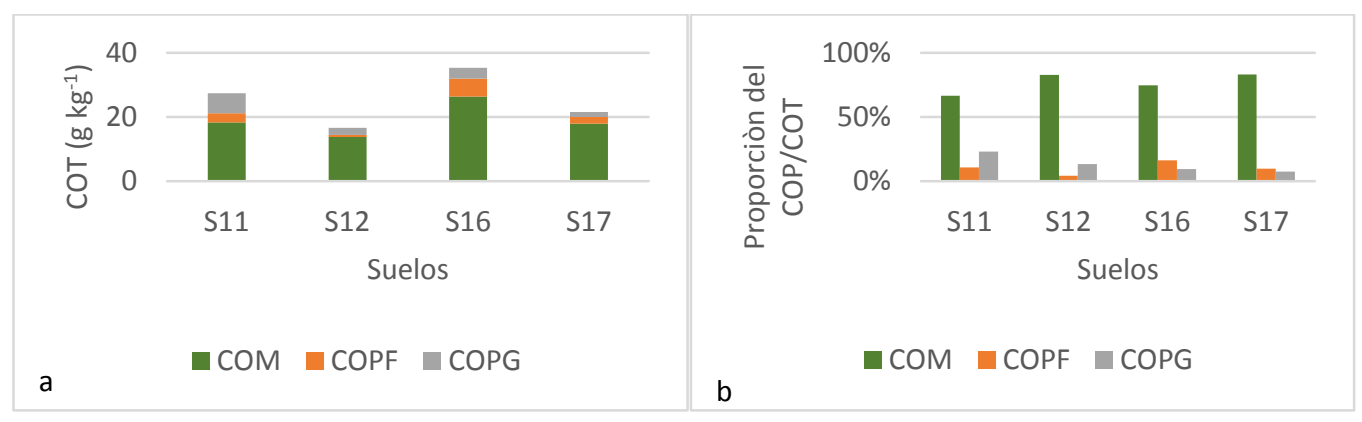

Figura 3.9 a. Carbono orgánico total (COT) expresado por la suma de las fracciones de carbono orgánico particulado (COP). b Proporción relativa de las fracciones de COP en relación al COT. 


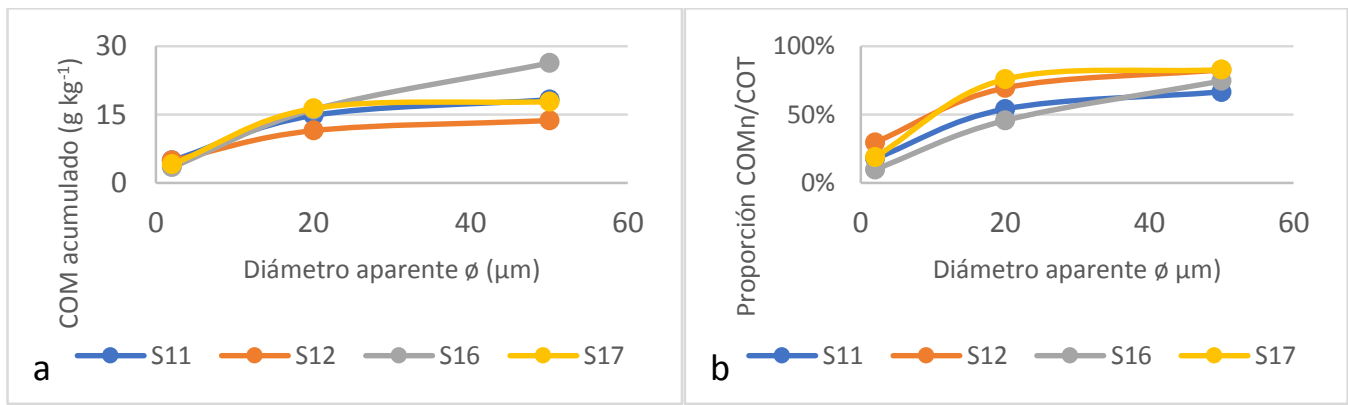

Figura 3.10 a Valor absoluto ( $\mathrm{kg}^{-1}$ ) del carbono orgánico asociado a la fracción mineral (COM) expresado como el valor acumulado de las fracciones de 2, 20 y $50 \mu \mathrm{m}$. b Proporción relativa de las fracciones del COM respecto del carbono orgánico total (COT).

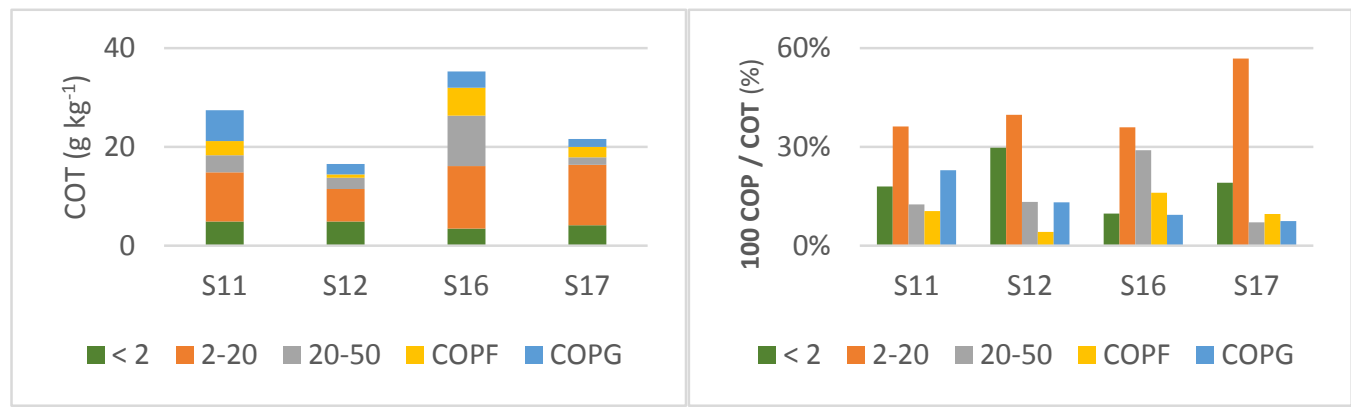

Figura 3.11a. Carbono orgánico total (COT) expresado por la suma de las fracciones de carbono orgánico particulado (COP) y las subdivisiones del COM. b Proporción relativa de las fracciones del COP y las subdivisiones del COM en relación al COT.

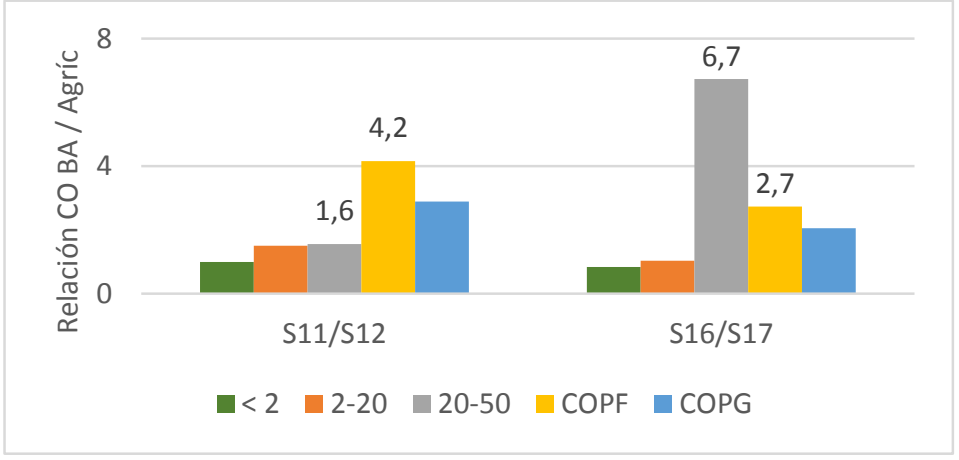

Figura 3.12 Cociente entre los valores de carbono orgánico aportado por cada fracción, entre el suelo bajo alambrado (BA) y el agrícola (Agríc)

\subsection{Análisis de la microestructuración en el tamaño arena}

Se planteó analizar la microestructuración para el tamaño arena en función del COM para todos los suelos estudiados. En la Figura 3.13a se representaron los valores de Aap, de la misma no se obtuvo una relación clara, los valores de los Grupos 1-2 y 3 muestran tendencias diferentes. El análisis por Grupo, realizado en los apartados anteriores (3.6) orienta sobre el porcentaje de microagregados en función del COM. En la Figura 3.13b, se observa que existe 
una relación significativa entre A/Aap y el COM. La misma es de tipo exponencial negativa, al aumentar el COM, se reduce la relación A/Aap. Esta reducción podría ocurrir por un aumento de las Aap, o una disminución de las A. Específicamente, los valores menores de esta relación corresponden a suelos con $\mathrm{A}$ es menor al $10 \%$ y el de las Aap es próximo a $50 \%$, el incremento del COM, aumentaría la microestructuración en este tamaño.

Con el análisis de los resultados, se arriba que el proceso de microestructuración en función del COM es diferente según sea la clase textural estudiada.

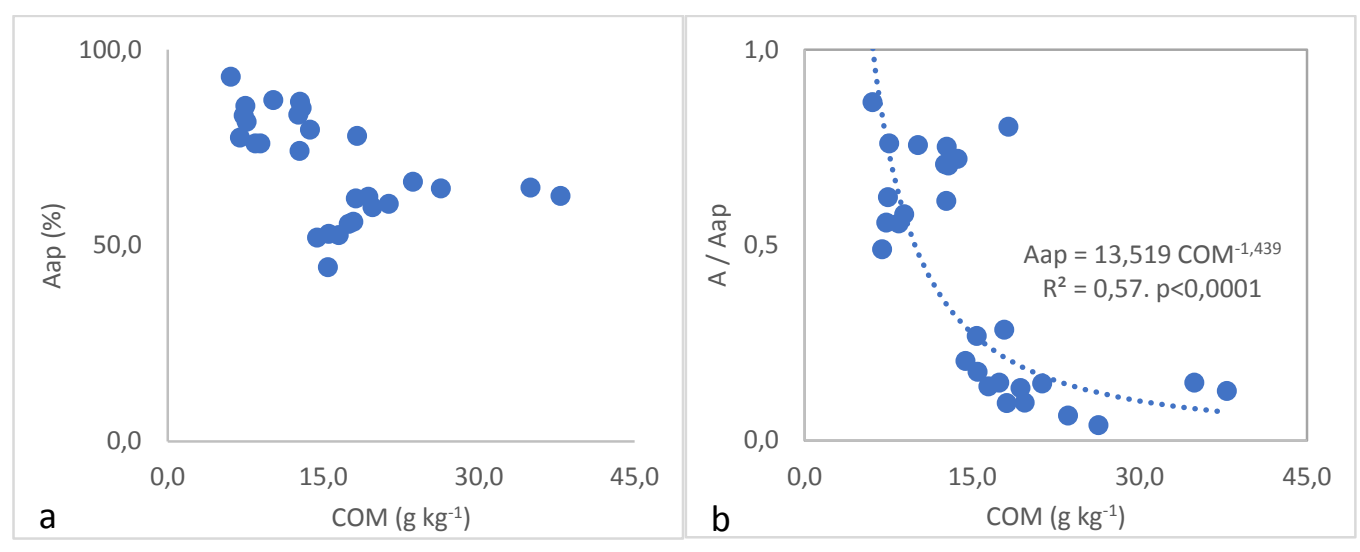

Figura 3.13.a Valores de arena aparente en función del carbono orgánico asociado a la fracción mineral (COM), para los Grupos 1, 2 y 3. Relación Arenas / Arenas aparentes en función del COM. 


\subsection{Imágenes al microscopio}

Se presentan los resultados de las fotografías tomadas al microscopio de muestras algunos de los suelos investigados. Las fotografías presentadas resultaron una selección de decenas de muestras observadas. Se emplearon aumentos de 40x, 100x, 250x y 400x.

En la Figura 3.14 se muestran las partículas de los suelos: S1 de la serie Arrecifes Argiudol Ácuico franco limoso, el S29 de la serie Saboya, otro Argiudol de textura franco y el S9 de la serie Norumbega un Hapludol Éntico, de textura franco arenoso.

Las fotos están ordenadas según los aumentos (40x, 100x, 250x y 400x) y corresponden al pretratamiento SMO. Se observan partículas que se encuentran, mayormente en el rango de las arenas muy finas a finas. Se aprecia que la eficacia del pretratamiento con $\mathrm{H}_{2} \mathrm{O}_{2}$ y calor fue elevada, dado que las partículas se ven limpias y sin pátinas de materia orgánica, especialmente en las arenas del S29 y S9. Más fotografías pueden observarse en: $\underline{\text { VinculoslSMO fotos }}$ 

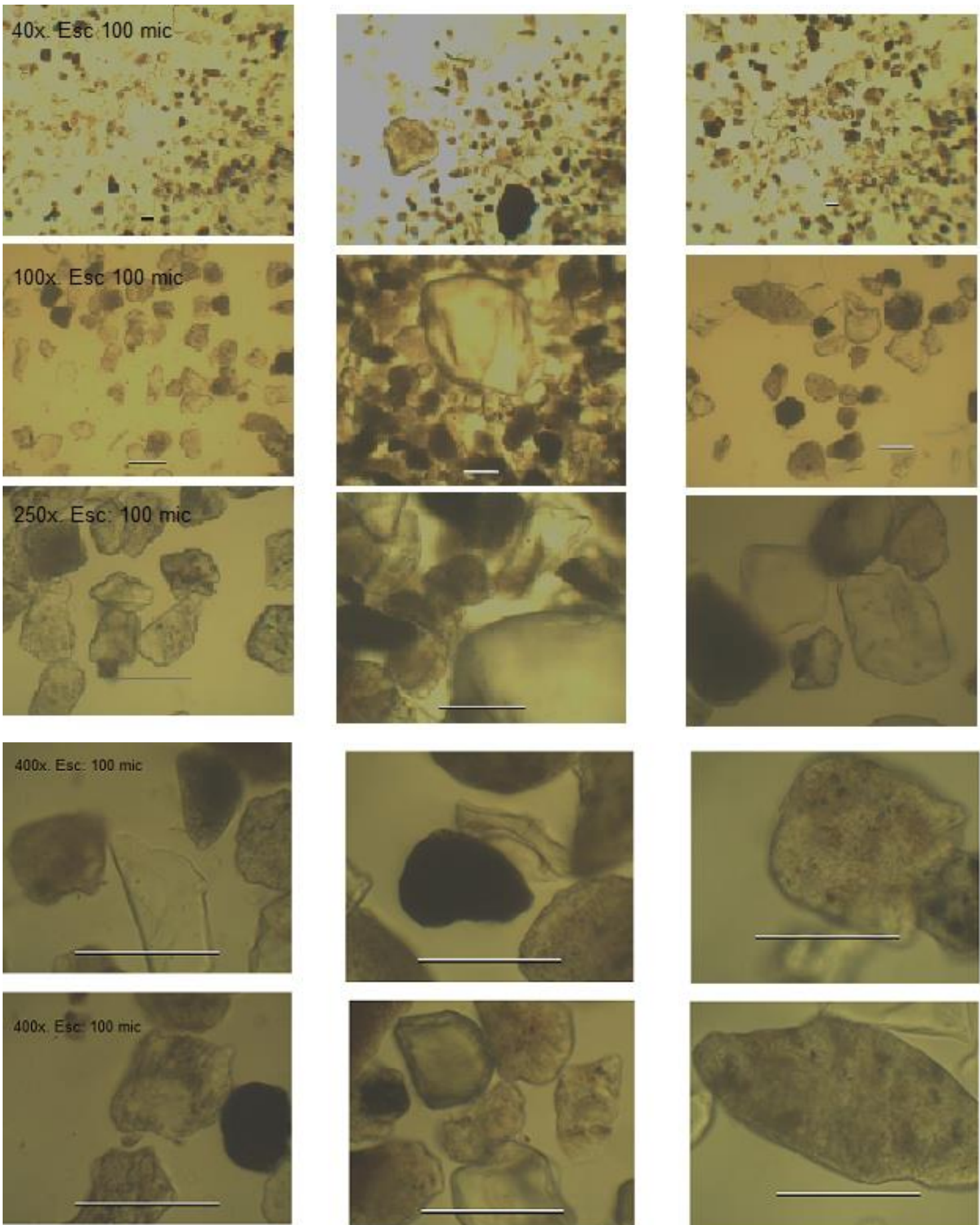

S1

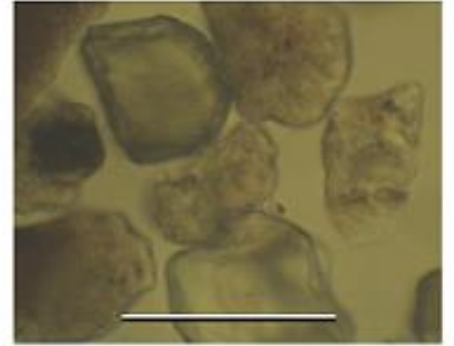

S29

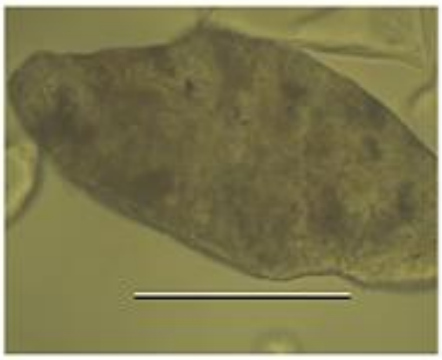

S9

Figura 3.14. Fotos tomadas sobre las arenas en el pretratamiento sin materia orgánica, obtenidas por tamizado. S1, serie Arrecifes; S29 serie Saboya y S9 serie Norumbega. Se adjunta escala gráfica.

En la Figura 3.15, las siguientes fotografías fueron tomadas sobre porciones de las muestras para sedimentar (arcilla + limo), con similares aumentos y presentadas en el mismo orden que en el caso anterior. En las fotografías se observa nuevamente la eficacia del pretratamiento, pues las partículas se aprecian sueltas, dispersas y sin restos orgánicos. No quedan en suspensión arenas, solo limos y arcillas. El S1, es el que contiene mayores 
porcentajes de limo y la suspensión se presentó más turbia. Los tamaños de las partículas visualizadas en las fotografías son menores a $50 \mu \mathrm{m}$.
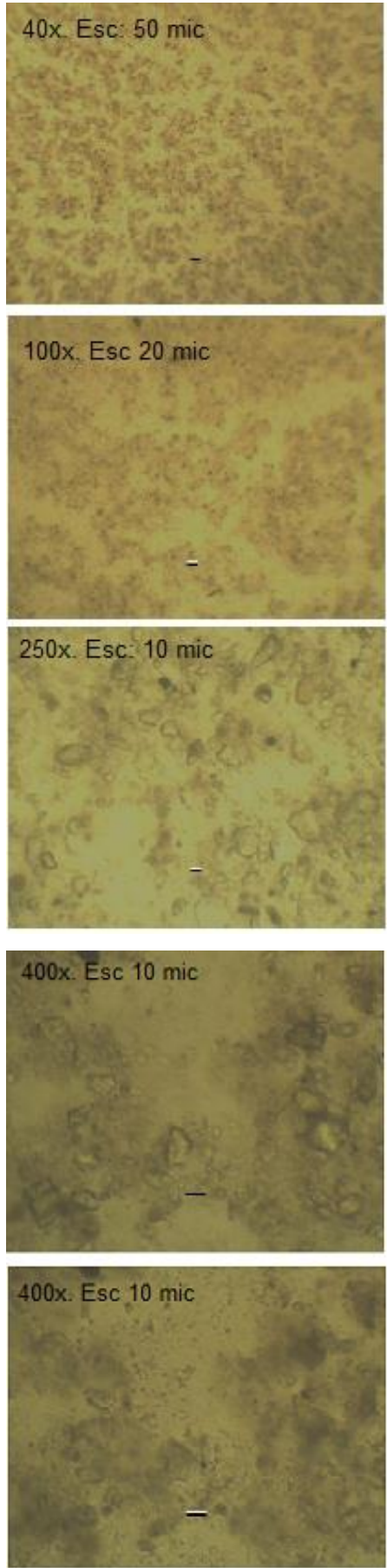

S1
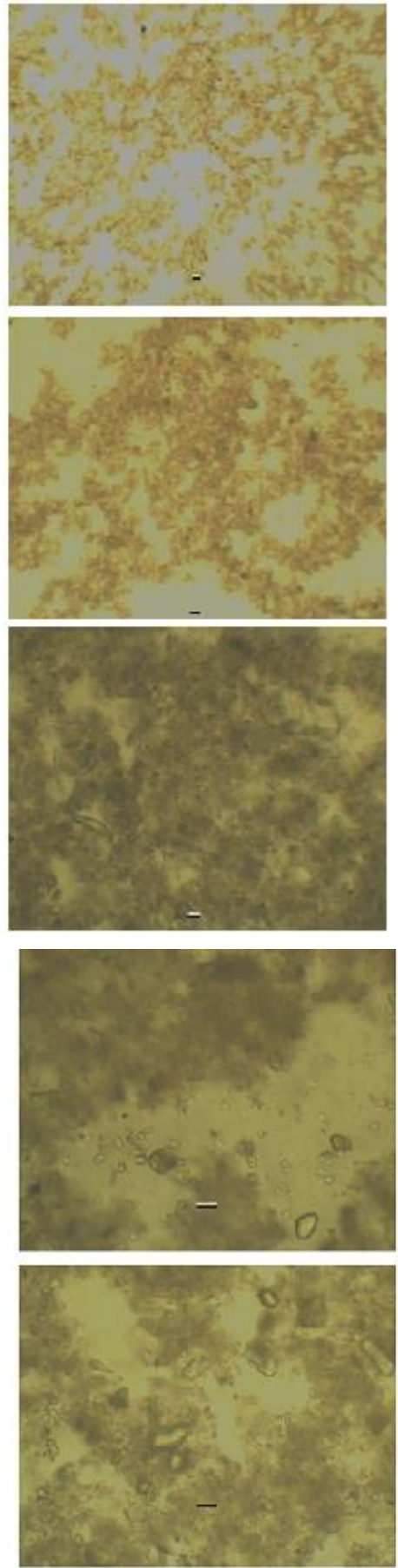

S29
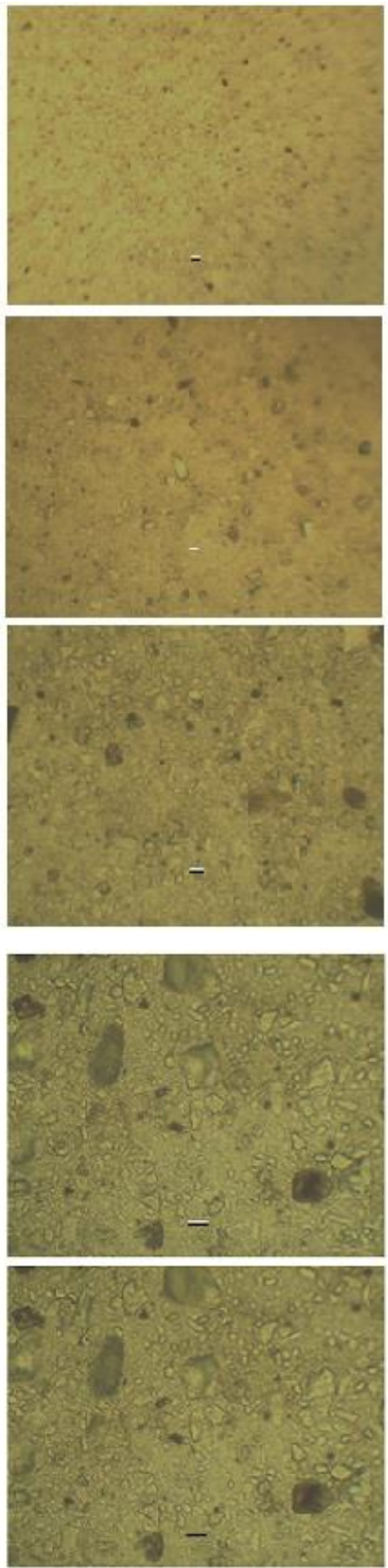

S9

Figura 3.15.-Fotos tomadas sobre la suspensión de limos y arcillas en el pretratamiento sin materia orgánica. S1, serie Arrecifes; 529 serie Saboya y 59 serie Norumbega. Se adjunta escala gráfica. 
Las siguientes fotografías, tomadas sobre las sedimentaciones $\mathrm{CMO}$, evidencian la microagregación de las partículas elementales por efecto de la MO. La variabilidad de formas y asociaciones entre partículas motivó duplicar la cantidad de fotografías por aumento, (Figura 3.16).

Puede apreciarse la agregación y tamaños de micropartículas mayores al pretratamiento SMO. Se observa en las fotos el color oscuro de las pseudopartículas, así como también la desaparición de la turbidez en la suspensión, producto de la agregación de las partículas elementales, principalmente en el tamaño arcilla y limo. En la fotografía del S1 40x, se ve un microagregado de más de $800 \mu \mathrm{m}$, pudiendo ser considerado un macroagregado según escala propuesta por Tisdall y Oades, (1982).

Con el incremento de los aumentos, se aprecia con más detalles las asociaciones entre partículas elementales aglutinadas por la MO. Para el suelo de la serie Norumbega se aprecia en los mayores aumentos cristales de arena limpios sin estar afectados por la MO. Más fotografías de microagregados pueden encontrarse en VinculoslCMO fotos 

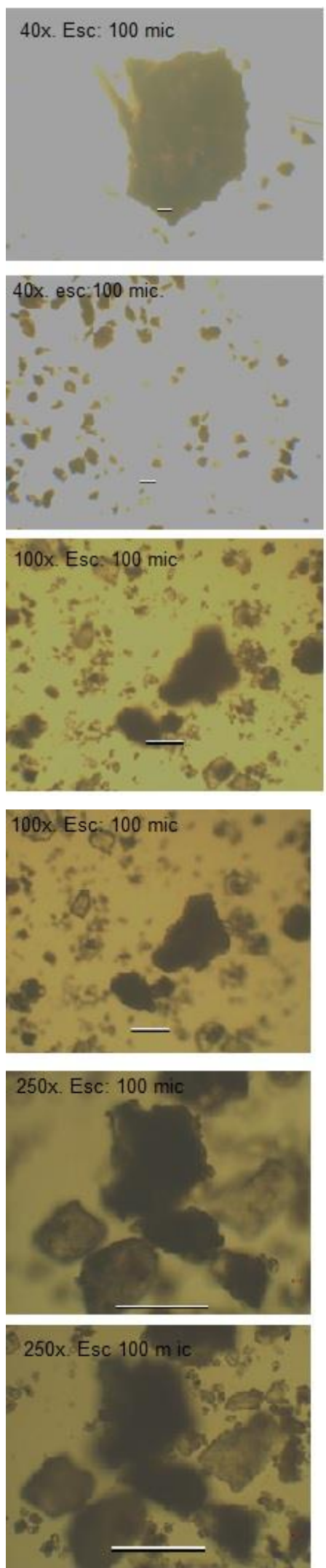

S1
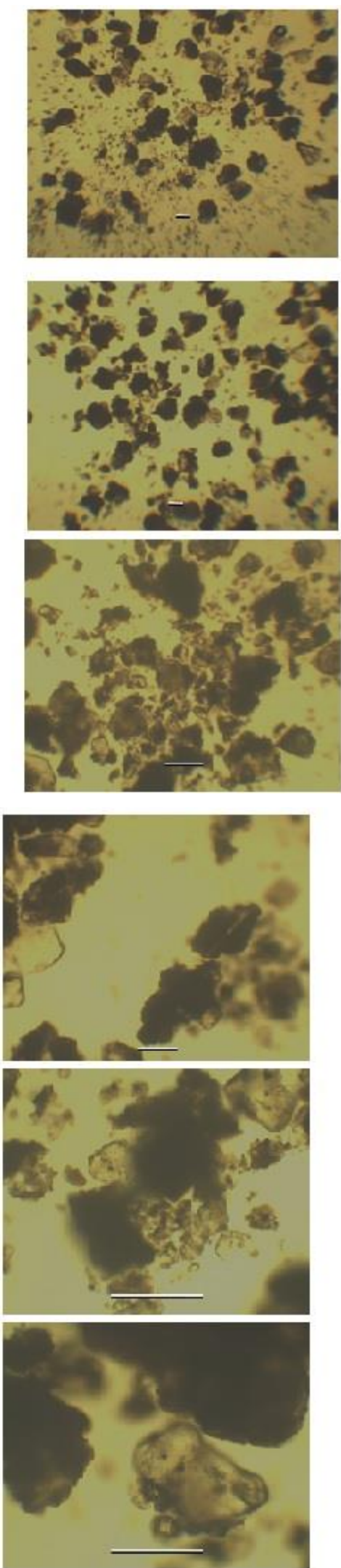

$\mathrm{S} 29$
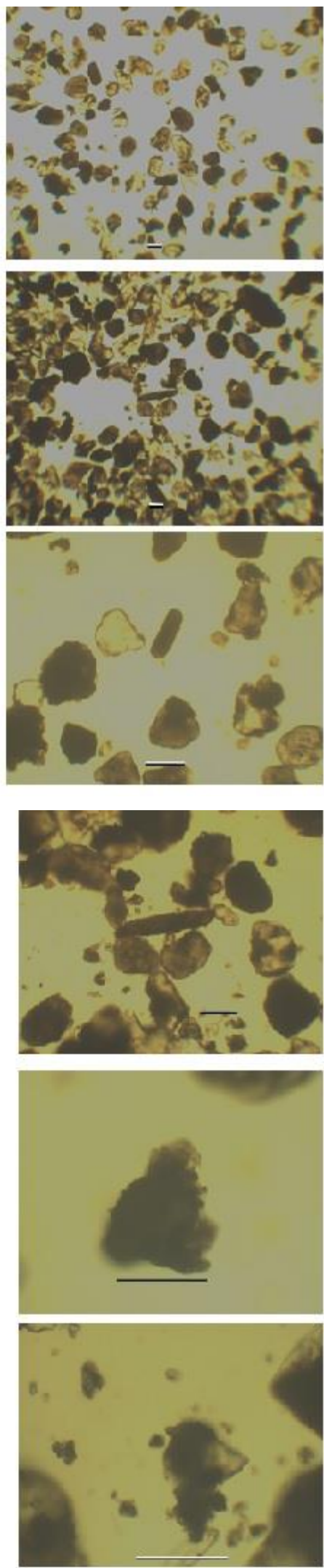

S9

Figura 3.16.-Fotos tomadas sobre la suspensión del pretratamiento con materia orgánica. S1, serie Arrecifes; S29 serie Saboya y S9 serie Norumbega. Se adjunta escala gráfica. En la serie Arrecifes, se aprecian los microagregados de tamaños superior a 100 mm. Las suspensiones de los tres suelos se observan límpidas por efectos de la microagregación de las partículas de menor tamaño. 

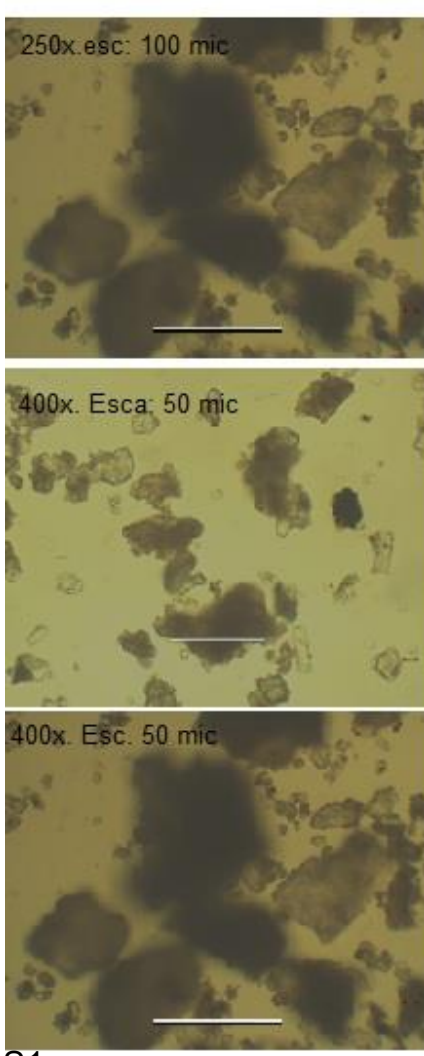

S1
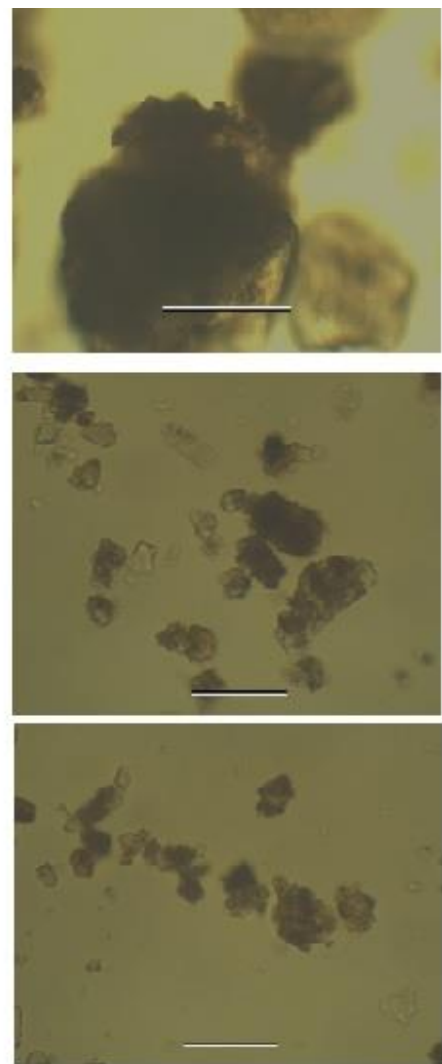

S29
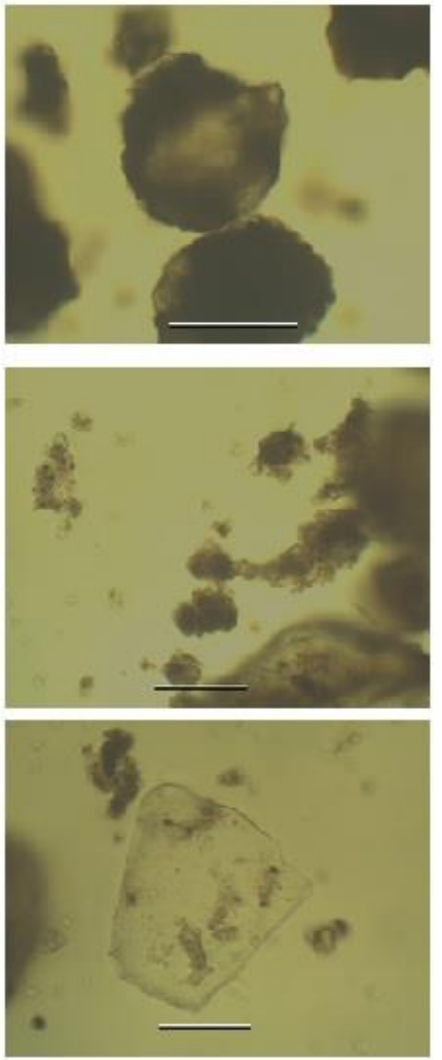

S9

Figura 3.17.(continuación)-Fotos tomadas sobre la suspensión del pretratamiento con materia orgánica. S1, serie Arrecifes; S29 serie Saboya y 59 serie Norumbega. Se adjunta escala gráfica. En la serie Arrecifes, se aprecian los microagregados de tamaños superior a $100 \mu \mathrm{m}$. Las suspensiones de los tres suelos se observan límpidas por efectos de la microagregación de las partículas de menor tamaño. 


\subsection{Discusión, fracciones reales y aparentes, microagregación}

En todos los suelos estudiados, independientemente del Grupo, cuando sedimentaron con materia orgánica, se observó un pasaje a clases texturales aparentes más gruesas, aumentando la proporción de arenas y disminuyendo las proporciones de limos y arcillas.

Al analizar el grado de la microagregación entre los grupos, encontramos que en el tamaño arena fue mayor en el G3 que en el G1, $(p<0,0001)$. El tamaño limo no mostró diferencias entre grupos $(p<0,26)$ y en el tamaño arcilla, el $G 1$ presentó una mayor agregación que el G3 $(p<0,0002)$. El G2, se comportó de manera similar al $\mathrm{G} 1$ en las comparaciones (Figura 3.17). El grado de agregación por efecto de la materia orgánica, proporcionalmente sería mayor cuando el porcentaje de la fracción real (arena, limo o arcilla) es menor, podría asociarse a la ley de los rendimientos decrecientes. Por ejemplo, como se dijo antes en el caso de la arcilla para el G1 los valores medios fueron de $13,9 \%$ (SMO) y 0,9\% para la arcilla aparente (CMO), mientras que el promedio de los cocientes (SMO/CMO) resultó de 18,7. En el caso del G3, los porcentajes promedios resultaron $25 \%$ (SMO) y $2,5 \%$ (CMO) mientras que la media del cociente fue de 11,0 (Figura 3.17). En otros trabajos se ha observado un comportamiento similar con relación al grado de cobertura de la superficie mineral por la materia orgánica (Gelati et al. 2009).

El aumento de tamaño aparente, producto de la microagregación, se manifestó en el incremento de la media de Phicmo. El Grupo 1 pasó $(p<0,0001)$ de un Phismo = 4,39 $(47,8 \mu \mathrm{m})$ "limo grueso" a un Phiсмо = 2,55 (171,3 $\mu \mathrm{m})$ "arena fina", mientras que el Grupo 3 ( $p<0,0001)$ pasó de un Phismo=5,78 $(18,2 \mu \mathrm{m})$ "limo mediano" a un Phiсмо =3,58 $(83,5 \mu \mathrm{m})$ "arena muy fina". El Grupo 2 mostró similar comportamiento, aunque menos manifiesto, con Phismo= 1,95 (259,2 $\mu \mathrm{m})$ (Arena mediana) у Phiсмо = 1,59 (333,3 $\mu \mathrm{m})$ "Arena mediana", sin cambiar de clase.

El G1, no mostró diferencias en el análisis del COT $(p=0,41)$ para los suelos "bajo alambrado" (16,66 $\left.\mathrm{g} \mathrm{kg}^{-1}\right)$ y agrícolas $\left(14,14 \mathrm{~g} \mathrm{~kg}^{-1}\right)$, ni con el COM $(p=0,20)\left(B A 12,08 \mathrm{~g} \mathrm{~kg}^{-1} \mathrm{y}\right.$ agrícola 9,61 $\mathrm{g} \mathrm{kg}^{-1}$ ). En este grupo, el porcentaje de las fracciones aparentes no se relacionaron significativamente con el COM - COT, no habría diferencias en el grado de microestructuración entre los suelos de la condición "bajo alambrado" o "agrícola".

En el G3, el COT se diferenció para los suelos bajo alambrado $\left(33,5 \mathrm{~g} \mathrm{~kg}^{-1}\right)$ respecto de los agrícolas $\left(19,7 \mathrm{~g} \mathrm{~kg}^{-1}\right)(p=0,01)$. Al analizar las fracciones de COP, el COM y el COPF mostraron diferencias mientras que el COPG no las tuvo. EI COM, diferenció los suelos "bajo alambrado" $\left(26,16 \mathrm{~g} \mathrm{~kg}^{-1}\right)$ y agrícola $\left(16,47 \mathrm{~g} \mathrm{~kg}^{-1}\right)$ con un $(\mathrm{p}=0,005)$. El COPF obtuvo para la condición bajo alambrado $\left(3,8 \mathrm{~g} \mathrm{~kg}^{-1}\right)$ respecto de la agrícola $\left(1,5 \mathrm{~g} \mathrm{~kg}^{-1}\right)$ y $(p=0,02)$. El COPG no mostró diferencias $(p=0,15)$. Las relaciones entre las fracciones aparentes y el COM fueron más fuertes que respecto del COT (Tabla 3.6).

Del análisis conjunto de la microestructuración en la relación A/Aap se desprende que si bien aparece una tendencia general de aumentar las Aap cuando incrementa el COM, es 
necesario considerar la clase textural, puesto que este proceso mostró comportamientos diferentes según los Grupos. Los microagregados tamaño arena y limo en función del COM, mostraron comportamiento diferente según las clases texturales estudiadas (Figura 3.18a-b).

Tabla 3.6 Valores de p en el cuerpo de la tabla para las fracciones aparentes y las diferentes fracciones de carbono analizadas.

\begin{tabular}{cccc} 
& Arena ap & Limo ap & arcilla ap \\
\hline COM & 0,0034 & 0,0018 & 0,053 \\
COT & 0,019 & 0,012 & 0,121
\end{tabular}

Oades y Waters (1991) establecieron un orden jerárquico en la estructura de los suelos, la cual presenta macroagregados $(>0,25 \mathrm{~mm})$ y microagregados $(<0,25 \mathrm{~mm})$. La estabilidad de los primeros varía con los cambios de contenido de $\mathrm{MO}$ y con las prácticas de manejo, debido a que los agregados son estabilizados transitoriamente por agentes como raíces, hifas y polisacáridos derivados de plantas y microorganismos (Bronick y Lal, 2005). El aumento en las operaciones de labranza de los suelos disminuye el carbono en los macroagregados (Six et al. 2000). Además, la MO joven y más lábil es contenida principalmente en los macroagregados y en menor proporción en los microagregados (Jastrow et al.1996). En cambio, la estabilidad de los microagregados es menos sensible a las prácticas de manejo y al contenido de MO (Tisdall y Oades, 1982).

Se conoce, que la cantidad de COT, que puede almacenar un suelo mineral está limitada por la granulometría y las condiciones de manejo. Suelos arcillosos retienen más COT que suelos arenosos, a pesar de haber sido sometidos al mismo aporte de MO (Jenkinson, 1988; Amato y Ladd, 1992; Hassink, 1994). El rango de COT encontrado en el G3 $\left(15,9-54,9 \mathrm{~g} \mathrm{~kg}^{-1}\right)$, más amplio que el de $\mathrm{G} 1\left(9,6-27,4 \mathrm{~g} \mathrm{~kg}^{-1}\right)$, podría explicarse por la mayor cantidad partículas finas en G3, (valores medios: arc: $25,0 \%$ y arc+Limo: $91,7 \%$ ) con respecto al $\mathrm{G} 1$ (valores medios arc: 13,9\% y arc+Limo: 46,1\%). En estas condiciones la MO puede asociarse y protegerse formando complejos arcillo húmicos, posibilitando mayor cantidad de estados de microagregación.

Siguiendo en el G3, con los resultados encontrados, puede afirmarse que en estos suelos Argiudoles, de clase textural franco limoso, con tenores de arena iguales o menores al $10 \%$, por efecto de la MO, se microestructuran partículas elementales - pseudopartículas y los suelos sedimentan como si se trataran de suelos franco arenosos.

Las fracciones aparente están significativamente relacionada con el contenido de COM. El tamaño arena está relacionado mediante una función potencial positiva, el tamaño limo por una función potencial negativa y el tamaño arcilla por una función lineal negativa. La función potencial explica mediante su derivada que las variaciones de arena y limos aparentes se van reduciendo con el incremento del COM, lo que es razonable, la superficie del mineral se va colmando con los primeros aportes de $\mathrm{CO}$ y aportes posteriores producen menos efecto que el anterior. 
Los microagregados, de menor tamaño acumulan más carbono orgánico por unidad de masa, por los efectos de protección de los complejos arcillo húmicos. A su vez, los suelos bajo alambrado, presentan mayor distribución del COT en las fracciones y subfracciones del COP. Los suelos agrícolas, con menor contenido de COT, donde la cantidad y la pérdida de formas reactivas de $\mathrm{CO}$, presentes en los microagregados mayores, hacen que estos no resistan el pretratamiento de dispersión, acumulándose la masa en los tamaños inferiores <2 y $2-20 \mu \mathrm{m}$.

Las prácticas de manejo, influyen en el contenido de COT- COM del suelo por lo tanto también en la microestruturación, para los suelos, franco-limosos, se puede afirmar que, la pérdida de CO produce variación de la textura aparente, reduciendo la proporción de microagregados tamaño arena, aumentando la de tamaño limo, esto implicaría una limitación en la formación de macroporos que tendría relevancia en la funcionalidad del suelo, por ejemplo, en el movimiento de fluidos. 

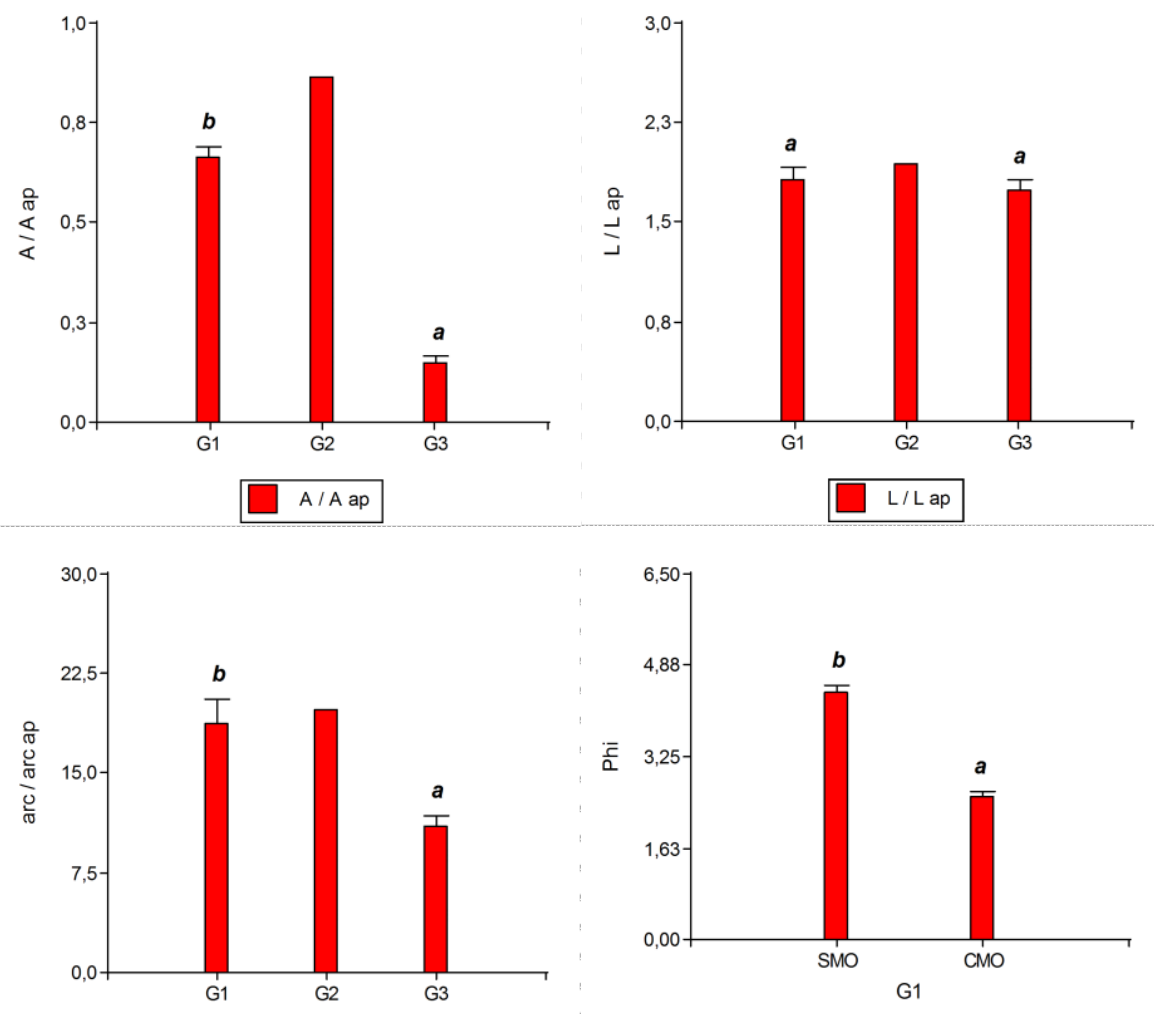

arc / arc ap

$\square$ Phi
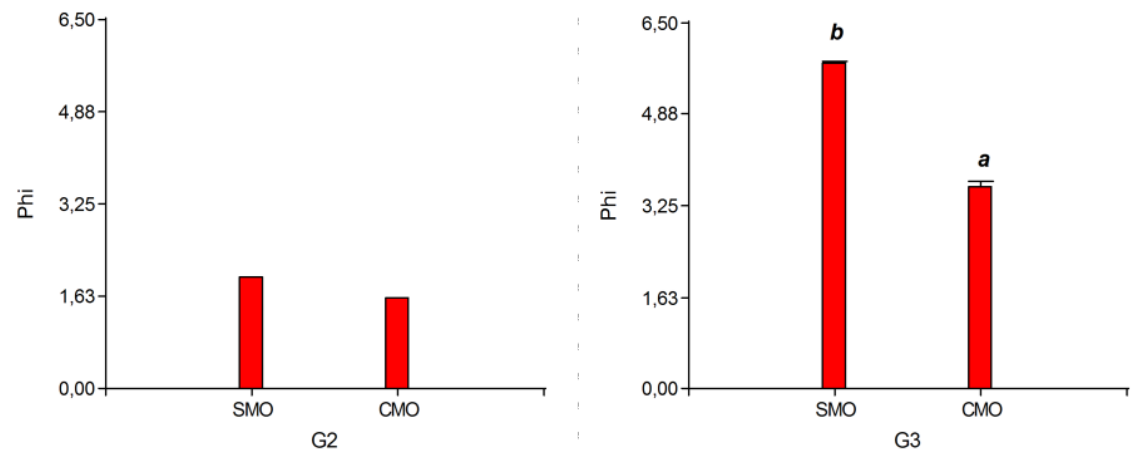

$\square$ Phi

Figura 3.18. Relaciones entre las fracciones reales y aparentes para los tamaños arena, limo y arcilla. Comparación del valor de Phi para los Grupos 1, 2 y 3 según el tratamiento sin materia orgánica (SMO) y con materia orgánica (CMO) 

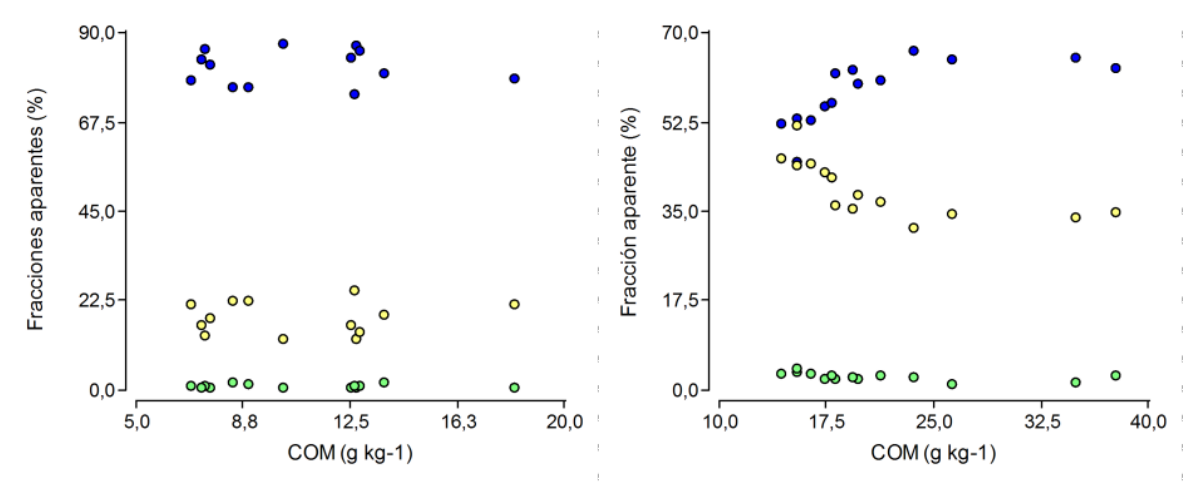

a

A ap $\bigcirc$ L ap $\bigcirc$ arc ap

b

A ap $\bigcirc$ L ap $\bigcirc$ arc ap

Figura 3.19. Relación entre fracciones aparentes de arena limo y arcilla en función del COM (a) para el G1 y (b) para el G3

\subsection{Conclusiones sobre la microestructuración}

La materia orgánica, microestructura los suelos, modificando la clase textural aparente, favoreciendo la aparición de microagregados de tamaños que beneficiaría el funcionamiento del suelo, por ejemplo, en el intercambio de fluidos.

Una misma fracción aparente de limo o arena, muestra comportamientos diferentes respecto del carbono orgánico asociado a la fracción mineral según se trate de suelos franco limosos o más gruesos que franco.

\subsection{Alcance de la metodología y propuesta de futuras investigaciones}

Continuar investigando la relación de las fracciones aparentes de arena y limo en clases texturales francos y más gruesos, en función de la materia orgánica para profundizar sobre los mecanismos de microagregación y sus consecuencias.

El método de textura aparente y las relaciones asociadas a la microestructuración podrían utilizarse como componente de un Índice de calidad físico químico, para lo cual sería necesario incorporarle el componente de la calidad de la materia orgánica, asociada a los microagregados puesto que involucra las asociaciones entre la fracción mineral y orgánica que cumplen un rol fundamental en la funcionalidad y calidad del suelo. 
Resultados: el modelo fractal y su aplicación al análisis de la distribución de tamaño de partículas

Capítulo 4 


\section{Modelo fractal}

\subsection{Estudio del modelo}

Desde hace varios años se viene utilizando la matemática fractal en el estudio de sistemas complejos, como es el caso del suelo. El modelo fractal aplicado a la DTP tuvo su auge en la década del 90, cuando varios investigadores se avocaron al estudio de esta característica del suelo (Bartoli et al. 1991; Tyler y Wheatcraft, 1992; Bitelli et al. 1999; Millan et al. 2003, Pachepsky y Rawls, 2004; Filgueira et al. (2003, 2006); Stanchi et al. (2006, 2008), Behzad et al. 2015).

Matemáticamente, la autosimilitud conlleva a una relación potencial entre el tamaño y las unidades de medición o escala (Sander, 1986; Tyler y Wheatcraft, 1989; Young y Crawford, 1991). Si la masa acumulada de partículas en función del diámetro sigue una función potencial, su distribución podría interpretarse mediante el modelo fractal de fragmentación, teniendo en cuenta algunas consideraciones. Como se mencionó en la materiales y métodos, la Ecuación 2.10 del modelo fractal expresada en relación de masas y diámetros, resulta ser fundamental para el análisis de las DTP.

$$
\frac{\mathrm{M}_{\mathrm{p}}}{\mathrm{M}_{\mathrm{T}}}=\left(\frac{\emptyset_{\mathrm{p}}}{\emptyset_{\mathrm{M}}}\right)^{3-\mathrm{D}} \therefore \mathrm{M}_{\mathrm{p}}=\frac{\mathrm{M}_{\mathrm{T}}}{\left(\emptyset_{\mathrm{M}}\right)^{3-\mathrm{D}}}\left(\emptyset_{\mathrm{p}}\right)^{3-\mathrm{D}}=\mathrm{C} \emptyset_{\mathrm{p}}^{3-\mathrm{D}}
$$

Dónde, Mp es la masa parcial acumulada hasta el tamaño $\emptyset_{p}(\mu \mathrm{m})$, MT es la masa total acumulada que muestra comportamiento fractal y alcanza el diámetro máximo $\varnothing_{\mathrm{M}}(\mu \mathrm{m})$ la DTP o DTM, D es la dimensión fractal de fragmentación y C una constante.

\subsection{Información que brinda el modelo}

En el modelo, brindan información ambos parámetros, aunque habitualmente solo se ha destacado el estudio de la dimensión fractal de fragmentación $D$.

El parámetro $C$ da idea del contenido de arcilla del suelo como así también del $\emptyset_{M}(\mu \mathrm{m})$ hasta el cual ajusta el modelo. Cuando $C$ es bajo, e.g., menor a diez, el contenido de arcillas del suelo es bajo y se espera un $\emptyset_{M}(\mu \mathrm{m})$ mayor, esto ocurre en suelos como los franco-arenosos. A medida que los suelos son más finos, el contenido de arcilla aumenta junto al valor de $\mathrm{C}$ y disminuye el valor $\emptyset_{M}(\mu \mathrm{m})$ como ocurre en los suelos franco limosos. Los suelos gruesos tienen D pequeñas, próximas a 2,4 mientras que suelos finos tienen valores de $D$ próximos a 2,6-2,8.

Del modelo se desprende una relación entre $C$ y $D$, a medida que se incrementa la $D$, el escalamiento es cada vez más pequeño y aumenta el valor de $C$ (como en suelo franco limosos). Si $D$ disminuye, el escalamiento es más grosero y el valor de $C$ es menor, como ocurre en suelos franco arenosos. 
El modelo tiene implícita otra consideración: si el contenido de arenas es bajo, (5 a 10\%), resulta un valor de "C" medio a alto y un diámetro máximo de arenas en el rango de las arenas muy finas. Al incrementarse la cantidad de arenas, aumenta el $\varnothing_{T}$ de la DTP, estos conceptos pueden observarse en la Figura 4.1 del triángulo textural. 


\subsection{Deducciones a partir del modelo}

4.3.1 Funciones de igual dimensión fractal (Iso D) e igual diámetro máximo (Iso $\varnothing_{T}$ ), para la tierra fina

A partir del modelo fractal y de la identidad textural del suelo, expresando la masa de los componentes arena, limo y arcilla en porcentaje, resulta:

$$
m_{A}+m_{L}+m_{\text {arc }}=m_{T}=100 \%
$$

$\mathrm{m}_{\mathrm{A}}=$ masa porcentual de arena

$\mathrm{m}_{\mathrm{L}}=$ masa porcentual de limo

$m_{\text {arc }}=$ masa porcentual de arcilla

$\mathrm{m}_{\mathrm{T}}=$ masa porcentual total $=100 \%$

De la Ecuación 2.10, para la fracción arcilla:

$$
\frac{m_{p}}{m_{T}}=\left(\frac{\emptyset_{p}}{\emptyset_{T}}\right)^{3-D} \rightarrow \quad \frac{m_{\text {arc }}}{100}=\left(\frac{\emptyset_{\text {arc }}}{\emptyset_{T}}\right)^{3-D} \rightarrow m_{\text {arc }}=100\left(\frac{\emptyset_{\text {arc }}}{\emptyset_{T}}\right)^{3-D} \quad \text { Ecuación 4-2 }
$$

Para las fracciones arcilla más limo:

$$
\frac{m_{a r c}+m_{L}}{100}=\left(\frac{\phi_{L}}{\phi_{T}}\right)^{3-D} \rightarrow m_{a r c}+m_{L}=100\left(\frac{\phi_{L}}{\phi_{T}}\right)^{3-D}
$$

Ecuación 4-3

Reemplazando Ecuación 4.3 en la Ecuación 4.1:

$$
m_{A}=100-\left(m_{\text {arc }}+m_{L}\right)=100-100\left(\frac{\phi_{L}}{\emptyset_{T}}\right)^{3-D}
$$

Ecuación 4-4

Y de esta:

$$
100-m_{A}=100\left(\frac{\phi_{L}}{\emptyset_{T}}\right)^{3-D}
$$

Ecuación 4-5

dividiendo miembro a miembro la Ecuación 4.5 con la Ecuación 4.2

$$
\frac{100-m_{A}}{m_{\text {arc }}}=\left(\frac{\emptyset_{L} \emptyset_{T}}{\emptyset_{\text {arc }} \emptyset_{T}}\right)^{3-D}
$$

\subsubsection{Rectas Iso D. Ecuación}

$$
m_{\text {arc }}=\left(\frac{\emptyset_{a r c}}{\emptyset_{L}}\right)^{3-D}\left(100-m_{A}\right)
$$


Siendo la ordenada al origen, cuando $\mathrm{m}_{\mathrm{A}}=0$

$$
m_{\text {arc }}=100\left(\frac{\emptyset_{a r c}}{\emptyset_{L}}\right)^{3-D}
$$

\subsubsection{Función Iso Øт. Ecuación}

Despejando 3-D en la Ecuación 4.5 se obtiene:

$$
(3-D)=\frac{\log \left(1-\frac{m_{A}}{100}\right)}{\log \left(\frac{\phi_{L}}{\phi_{T}}\right)}
$$

Ecuación 4-7

y reemplazado esta expresión (Ecuación 4.7) en la Ecuación 4.6, se obtiene la función Iso Øт

$$
m_{\text {arc }}=\left(\frac{\emptyset_{\text {arc }}}{\emptyset_{L}}\right)^{\frac{\log \left(1-\frac{m_{A}}{100}\right)}{\log \left(\frac{\phi_{L}}{\phi_{T}}\right)}} \times\left(100-m_{A}\right) \quad \text { Ecuación 4-8 }
$$

\subsubsection{Ecuación para calcular la dimensión fractal teórica $D$}

A partir de la Ecuación 4.6, con los contenidos porcentuales de arena y arcilla, puede calcularse la dimensión fractal de fragmentación teórica.

$$
D=3-\frac{\log \left(\frac{m_{\text {arc }}}{100-m_{A}}\right)}{\log \left(\frac{\phi_{\text {arc }}}{\phi_{L}}\right)}
$$

Ecuación 4-9

La Ecuación 4.6 (Iso D) se graficó en el triángulo textural (Figura 4.1). Las líneas obtenidas unen texturas con diferentes valores de arena y arcilla, pero la misma $D$, mientras que la Ecuación 4.8 (Iso $\varnothing_{T}$ ) graficó las líneas con igual $\varnothing_{\text {T. }}$ Esta última función, define en el triángulo textural dos áreas; a la izquierda de $\varnothing_{T}=2000 \mu \mathrm{m}$, se ubican las combinaciones texturales de suelos potencialmente fractales mientras que a la derecha combinaciones texturales no fractales. Por definición, curvas Iso $\varnothing_{T}>2000 \mu \mathrm{m}$ no corresponderían a tierra fina y no podrían ser graficados en el triángulo textural. 


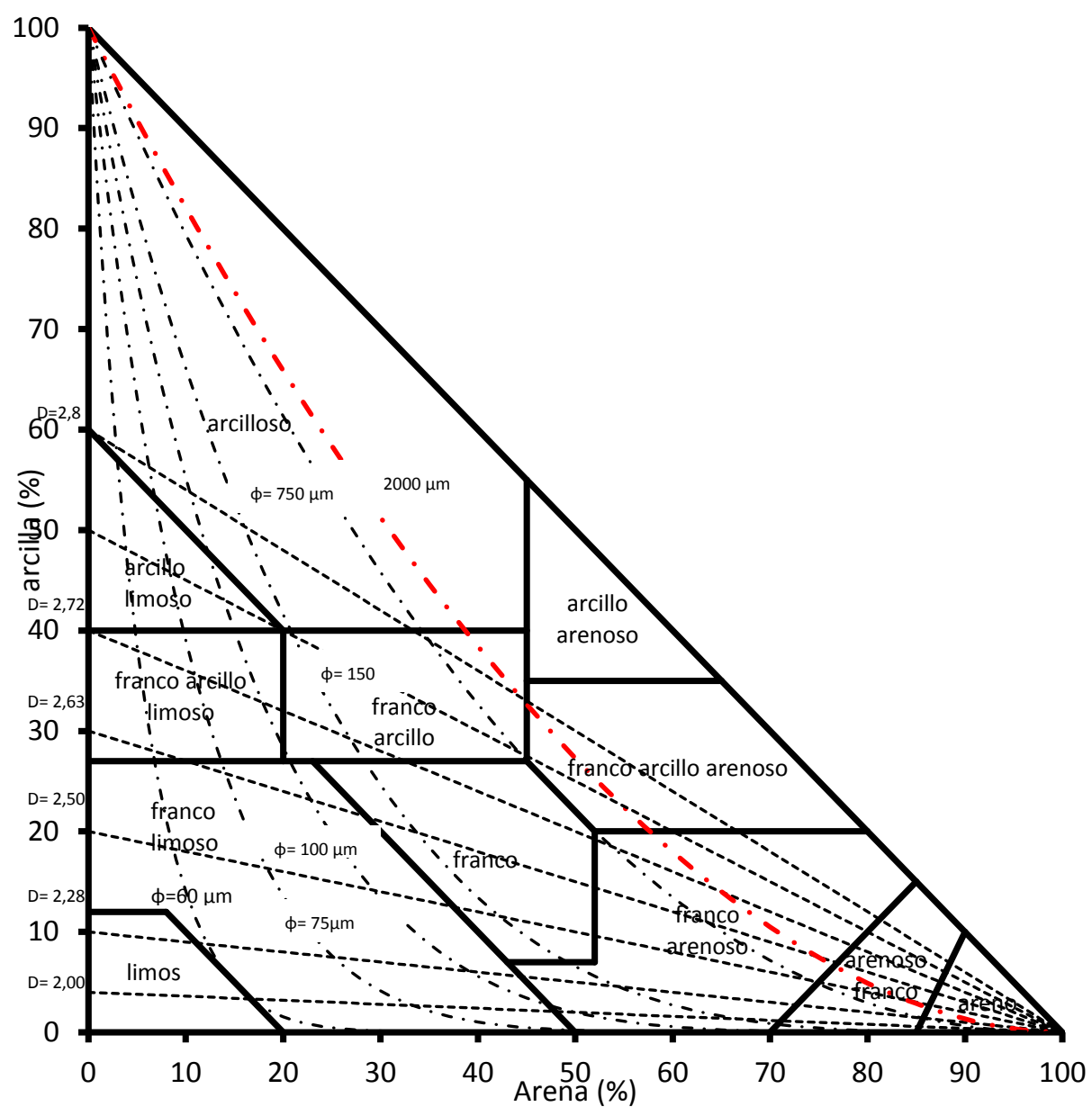

Figura 4.1. Triángulo textural, con las clases texturales tradicionales USDA. Funciones Iso D (---) e Iso $\varnothing_{T}$. (- -). La línea roja (Iso $\emptyset_{T}=2000 \mu \mathrm{m}$, divide el área del triángulo en suelos potencialmente fractales a la izquierda y suelos no fractales a la derecha de la misma

\subsubsection{Parámetros del modelo fractal en función del contenido de arcilla}

Del modelo puede deducirse, que la relación entre $D$ y el contenido de arcillas es logarítmica y queda definida por la siguiente expresión:

$$
D=3-\frac{\log \frac{m_{\text {arc }}}{100}}{\log \frac{2}{\bar{\phi}_{T}}}
$$

Ecuación 4-10

Distintas DTP con el mismo contenido de arcilla pueden tener diferentes D, como se observa en la Figura 4.2a, el valor de $\Phi_{\mathrm{T}}$, es el que da las diferentes curvas de la familia. 
Del modelo también se obtiene, en forma teórica el parámetro "C" en función del contenido de arcilla y la $\mathrm{D}$.

$$
C=m_{\text {arc }} 2^{D-3}
$$

Ecuación 4-11

El parámetro $C$, es una función lineal del contenido de arcilla y está afectado por la dimensión fractal de fragmentación. Las representaciones de $C$ y $D$ en función del contenido de arcillas pueden observarse en la Figura 4.2a-b.
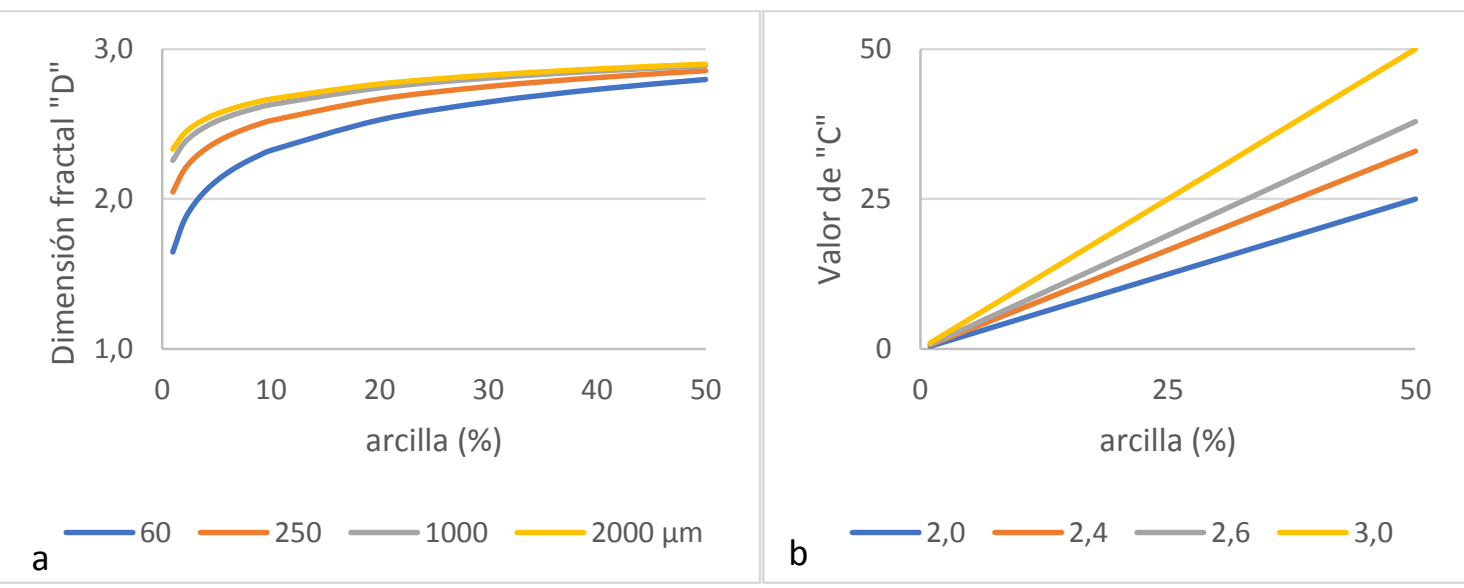

Figura 4.2. a. Dimensión fractal de fragmentación " $D$ " en función del contenido de arcilla para diferentes $\emptyset_{T}$. $b$ Valor de $C$ en función del contenido de arcillas para diferentes " $D$ ", ambas expresiones deducidas del modelo teórico.

\subsection{Estudio fractal de la distribución de tamaño de partículas de los suelos muestreados}

En este capítulo se trabajó con el modelo fractal aplicado a los grupos de suelos antes descriptos, se realizaron análisis grupales e individuales para los suelos de cada grupo, obteniéndose los parámetros del modelo fractal a partir de los datos experimentales.

\subsection{Grupo 1}

Al aplicarle el modelo fractal a este grupo, formado por suelos francos y franco arenosos, en el ajuste grupal e individual, se evidenció una tendencia en los residuos (Figura 4.3c), indicando que el modelo propuesto no resulta ser el mejor. Sin embargo, los ajustes fueron elevados, significativos y puede emplearse el modelo potencial linealizado, puesto que la regresión describe adecuadamente los valores experimentales. 


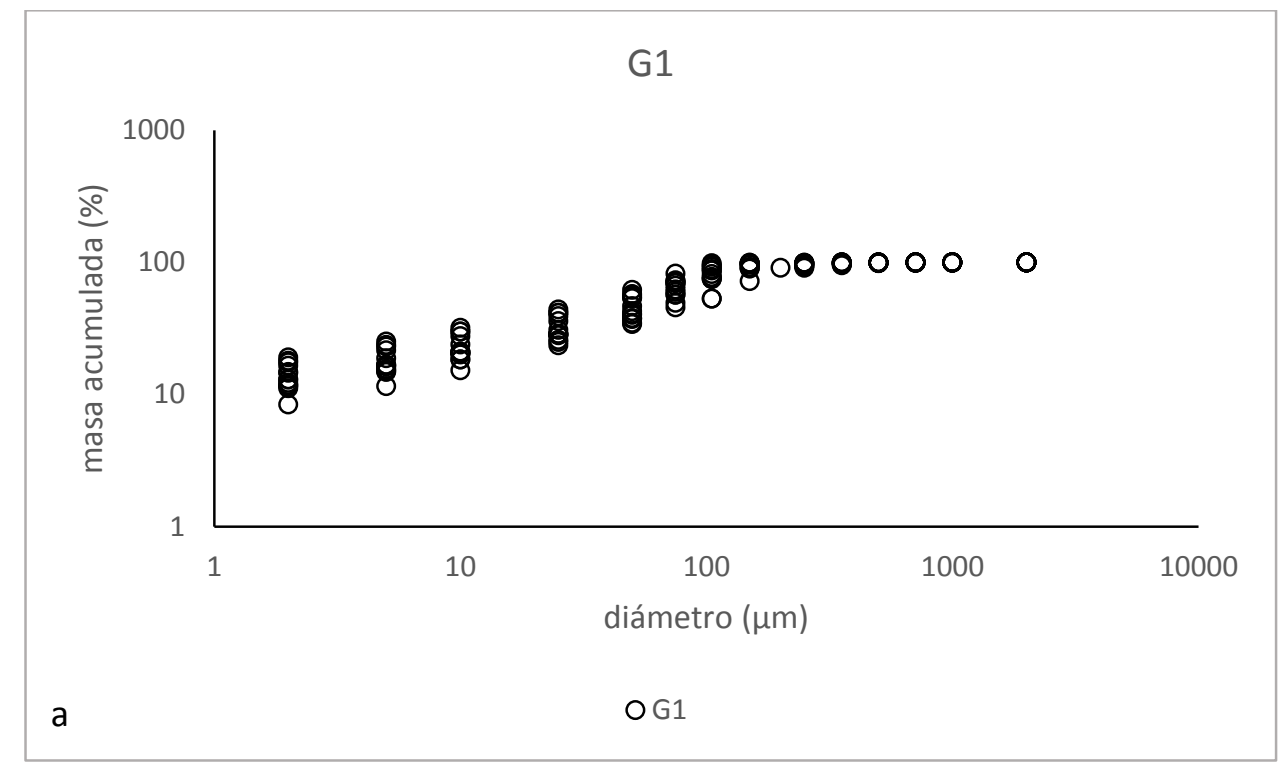

G1.2-75

G1.2-75
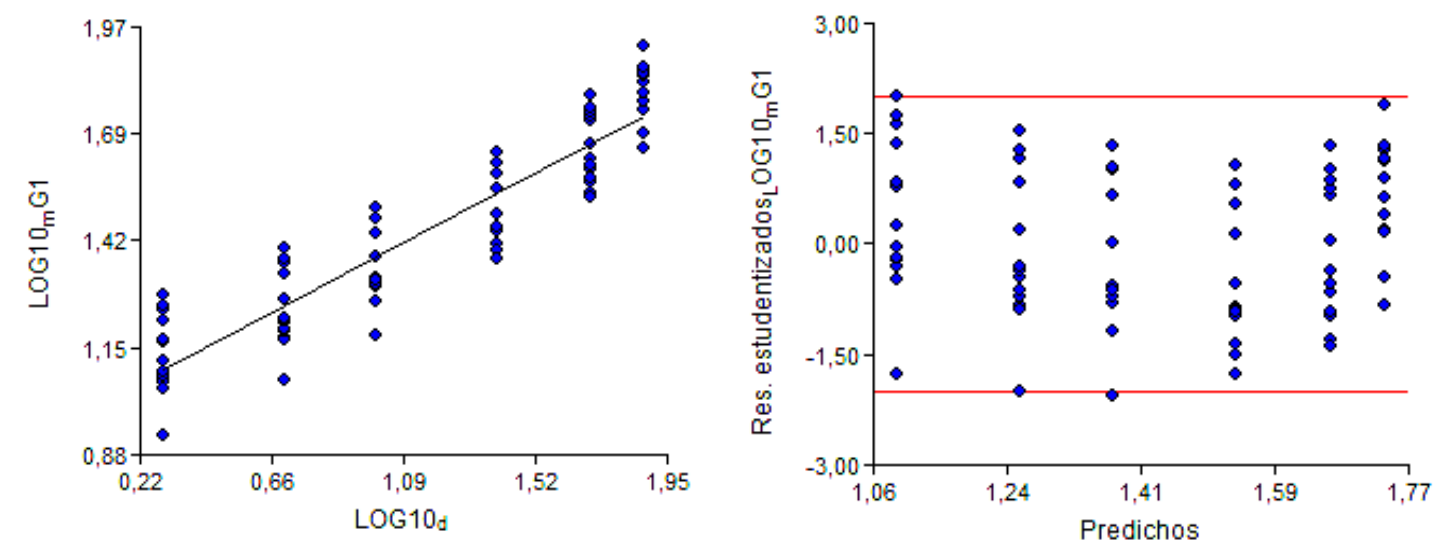

b

C

Figura 4.3, a. Distribución de tamaño de partículas completa del G1, de masa acumulada en función del diámetro, ambos ejes en escala logarítmica; $b$. Distribución de tamaño de partículas entre 2 -75 $\mu m$, ambos ejes en escala logarítmica; c. Distribución de residuos

\subsubsection{Subdivisión del G1 en G1 Franco - G1 Franco Arenoso}

En este grupo, los coeficientes de variación de las medias de arena, arcilla y Phi medio resultaron elevados, (18\%, $23 \%$ y $33 \%$ respectivamente) y con el objetivo de profundizar el análisis fractal de las DTP, se lo subdividió en dos (Figura 4.4), resultando el grupo 1 de suelos francos (G1F) y el grupo 1 de suelos franco arenosos (G1FA). 


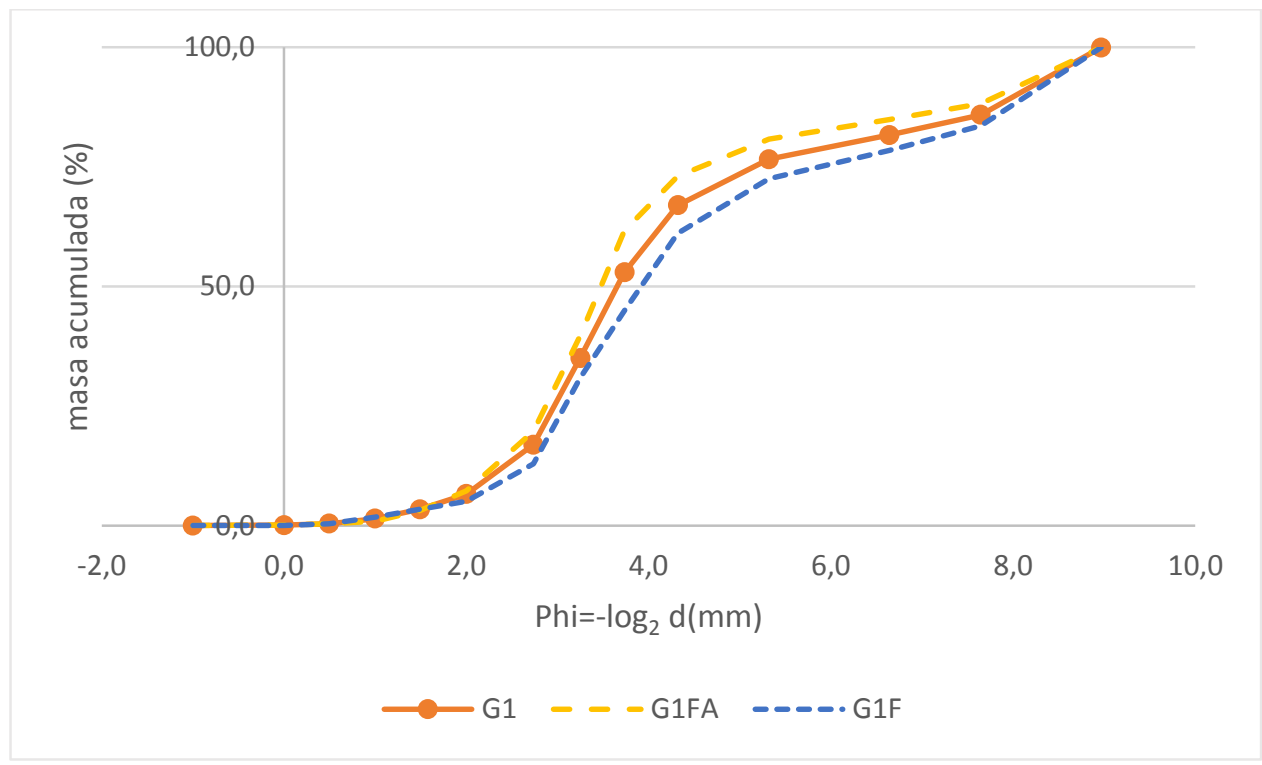

Figura 4.4. Masa acumulada en función del parámetro Phi para el G1 y los resultantes G1F y G1FA, luego de la separación.

\subsubsection{Grupo 1 franco. Análisis grupal}

Se formó con los suelos (S24, S25, S26, S27 y S28), provenientes de la Pampa Arenosa. La fracción dominante fue la arena con $44,9 \%$, le siguió el limo con $38,7 \%$ y por último la arcilla con $16,4 \%$. La DTP en la escala sedimentológica (Figura 4.5) obtuvo una media de $\mathrm{Phi}=4,74$ equivalente a $38,0 \mu \mathrm{m}$ y un desvío estándar de 2,19 , que lo califica como "limo grueso, muy pobremente seleccionado".

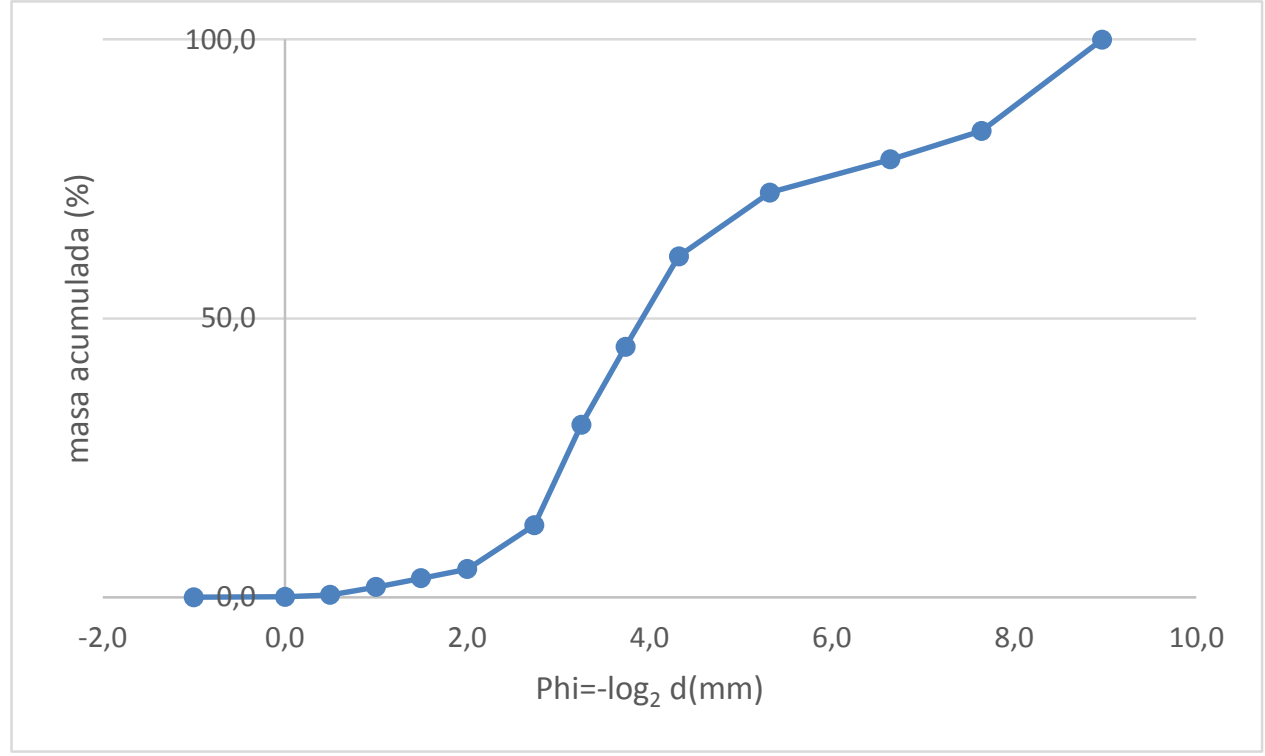

Figura 4.5. Distribución de tamaño de partículas de masa acumulada en función de Phi. 
Ajustando los valores de masa acumulada en función del diámetro, con el modelo fractal, se distinguen tres sectores, cada uno con diferente $C$ y D. (Figura 4.6).

Los puntos de inflexión entre dominios se obtuvieron matemáticamente resolviendo la intersección entre las rectas resultantes.

El primer sector abarca desde las arcillas hasta los $33 \mu \mathrm{m}$ (punto de inflexión), describiendo el $44 \%$ de la masa experimental. El segundo, de $33 \mu \mathrm{m}$ a $130 \mu \mathrm{m}$, describe hasta el $95 \%$ de la masa experimental (dominio desde el limo hasta arenas finas) y el tercer sector comprende desde las arenas finas hasta las gruesas con una $D$ próxima a 3 (comportamiento euclidiano).

Las expresiones del modelo, los parámetros del mismo y el ajuste para cada tramo se presentan a continuación y se muestran en la Figura 4.6.

Primer sector:

$$
\begin{gathered}
m 1 G 1 F(\%)=11,915 \emptyset^{0,366} \quad \mathrm{D} 1=2,634 \\
2 \leq \emptyset(\mu \mathrm{m}) \leq 33 \\
R^{2}=0,91 . p<0,001
\end{gathered}
$$

Segundo sector:

$$
\begin{gathered}
m 2 G 1 F(\%)=6,311 \emptyset^{0,558} \quad \mathrm{D} 2=2,442 \\
33 \leq \emptyset(\mu \mathrm{m}) \leq 130 \\
R^{2}=0,92 . p<0,001
\end{gathered}
$$

Tercer sector:

$$
\begin{gathered}
m 3 G 1 F(\%)=84,411 \emptyset^{0,025} \quad \mathrm{D} 3=2,975 \\
130 \leq \emptyset(\mu \mathrm{m}) \leq 1000 \\
R^{2}=0,52 . p<0,001
\end{gathered}
$$

Los suelos de este grupo, fueron descriptos hasta el $95 \%$ de la masa experimental empleando el modelo fractal sectorizado, siendo los tramos uno y dos los que aportaron información, mientras que el tramo tres no se consideró fractal por tener la $D$ próxima a 3.

Los parámetros estimados para el primer y segundo sector fueron coherentes con el marco teórico. La D1 resultó mayor que D2, lo que estaría indicando que el tramo uno se trata 
de un material más finamente particionado que el tramo dos, coherente con esto, la C1 resultó mayor que $\mathrm{C} 2$, lo que implica que el tramo uno tendría un $\varnothing \mathrm{T}$ menor que el tramo dos. El tramo uno abarca las arcillas, limos finos y parte de limos gruesos mientras que el dos describe parte de los limos más gruesos y arenas muy finas a finas. En este subgrupo, la inflexión ocurre dentro de los limos gruesos y el segundo sector incorpora los limos gruesos, las arenas muy finas y una porción de arenas finas.

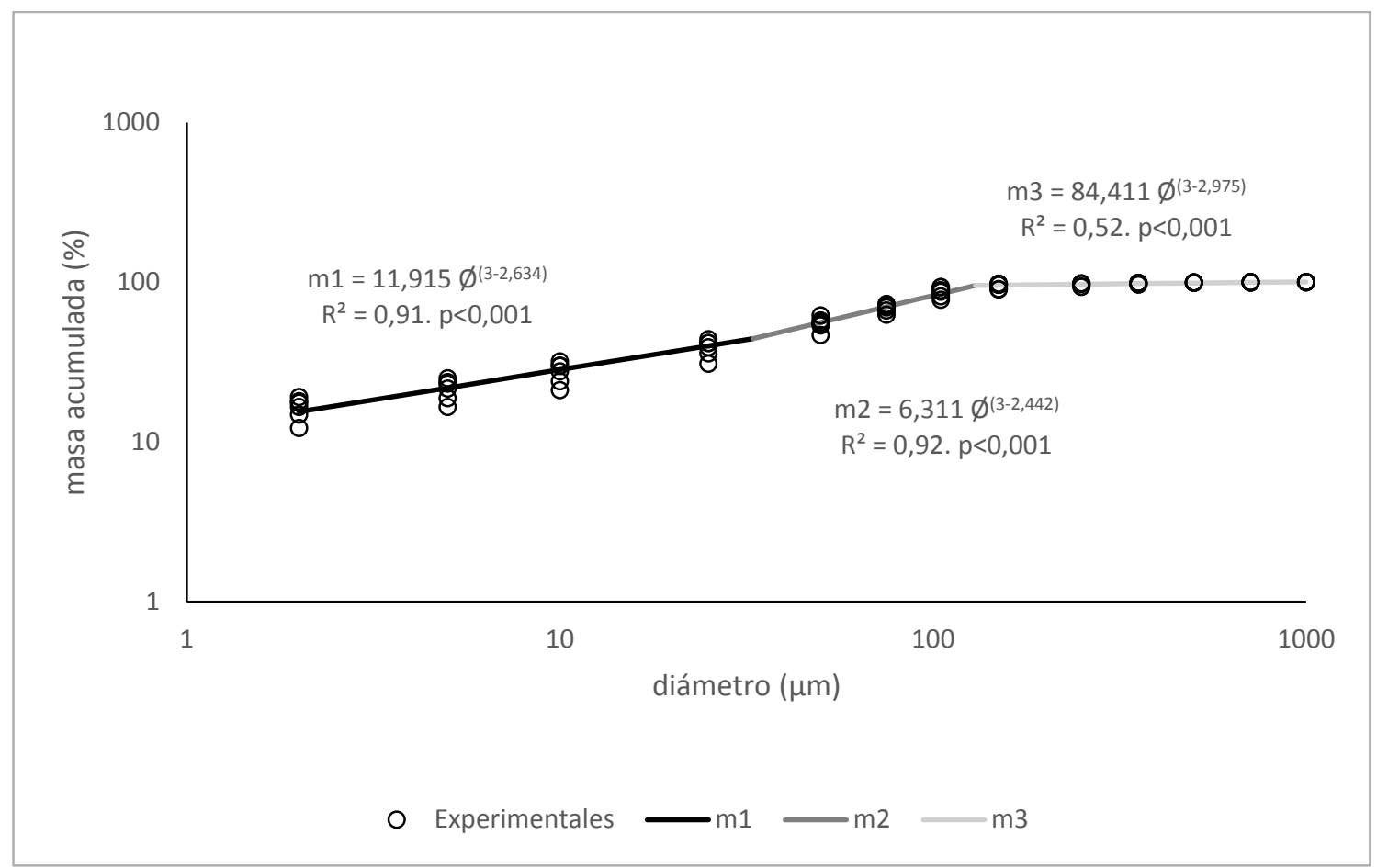

Figura 4.6. Modelo fractal para el G1F, acotado por sectores, valores experimentales observados en la distribución de tamaño de partículas, masa acumulada en función del diámetro.

\subsubsection{Ejemplo de análisis individual}

Para facilitar la exposición de los resultados, obtenidos mediante el modelado fractal de los suelos de este Grupo se desarrolla como ejemplo el análisis individual de uno de los mismos. Los valores correspondientes a los otros integrantes pueden consultarse en: VinculosIG1F CyD individuales.xlsx

Se describió el suelo S26, un Hapludol Éntico de la serie Bolivar, el mismo se caracterizó por una textura franca con $45,4 \%$ de arena, $38,0 \%$ de limo y $16,6 \%$ de arcilla. La curva de DTP en función de Phi obtuvo una media=4,81 equivalente a 35,7 $\mu \mathrm{m}$ y un desvío estándar de 2,19, que lo calificó como "limo grueso muy pobremente seleccionado"

La DTP de este suelo presentó los tres sectores definidos anteriormente, a los cuales se les ajustó el modelo fractal en cada sector. El modelo para el primer y segundo sector (m1 y m2), 
tienen características fractales mientras que el tercero (m3), correspondiente a las arenas de mayor tamaño y posee una $\mathrm{D}$ próxima a 3 .

Se presentan los valores correspondientes al modelo de cada sector y los mismos junto a los datos experimentales se graficados en la Figura 4.7.

Primer sector:

$$
\begin{aligned}
& m S 26(\%)=12,274 \emptyset^{0,369} \quad \mathrm{D} 1=2,631 \\
& 2 \leq \emptyset(\mu \mathrm{m}) \leq 32 \\
& R^{2}=0,99 . p=0,003
\end{aligned}
$$

Segundo sector:

$$
\begin{aligned}
& m 2 S 26(\%)=6,491 \emptyset^{0,553} \quad \mathrm{D} 2=2,447 \\
& 33 \leq \emptyset(\mu \mathrm{m}) \leq 132 \\
& R^{2}=0,99 . p=0,003
\end{aligned}
$$

Tercer sector:

$$
\begin{gathered}
m 3 S 26(\%)=87,519 \emptyset^{0,020} \quad \mathrm{D} 3=2,980 \\
132 \leq \emptyset(\mu \mathrm{m}) \leq 1000 \\
R^{2}=0,90 \cdot p=0,004
\end{gathered}
$$

En el análisis individual aparecen las mismas características que en el análisis grupal, pero se observó un mayor grado de ajuste. Este hecho se repitió en todos los suelos del Grupo.

La diferenciación de los rangos de aplicación del modelo permitió incorporar parte de las arenas. El comportamiento de las arenas descriptas con $\mathrm{m} 2$, a nivel grupal e individual, cuestiona a la bibliografía donde se informa que esta fracción acumulada no presentaría distribución fractal. 


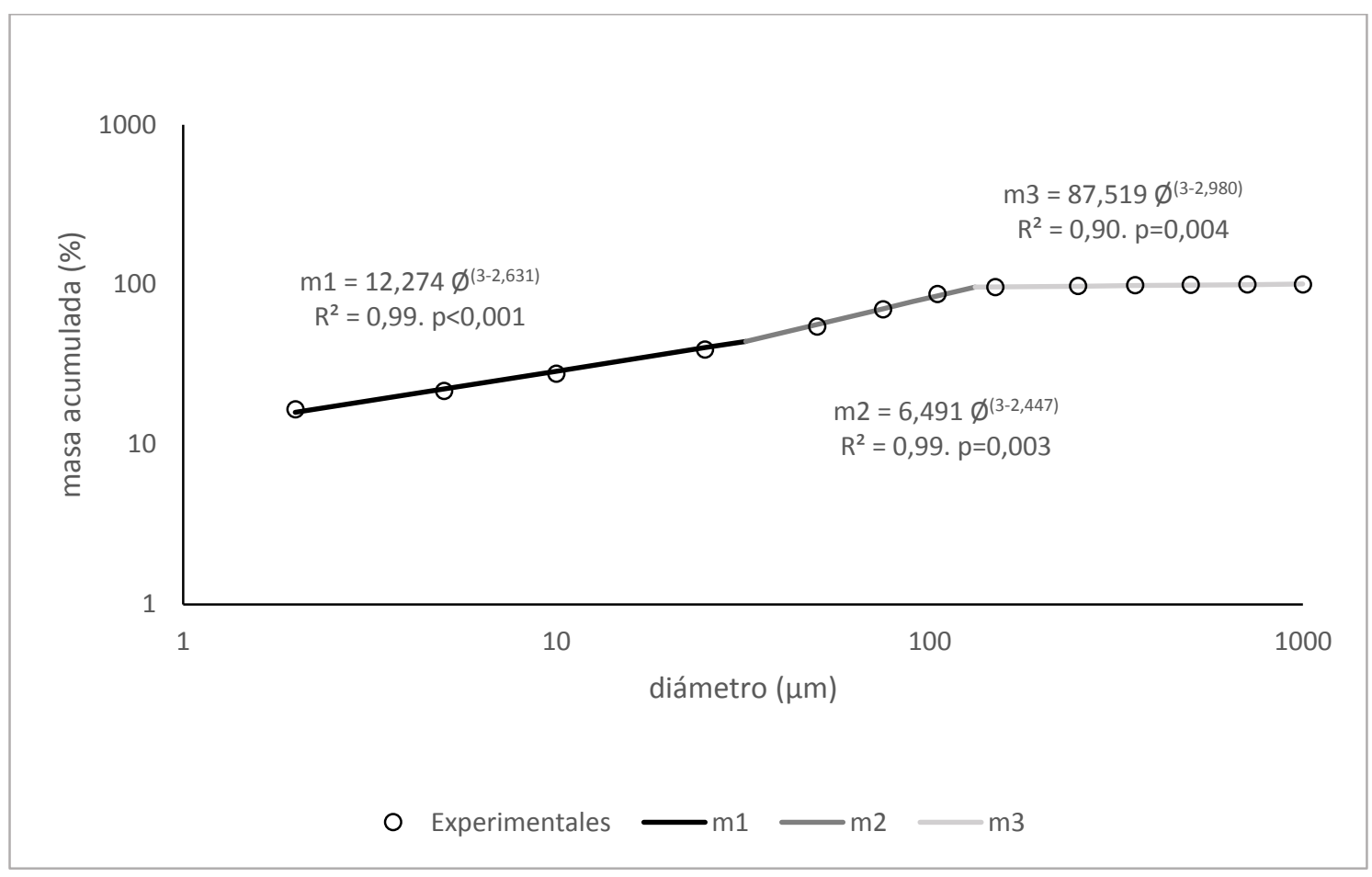

Figura 4.7. Modelo fractal acotado por sectores, ajustados particularmente al suelo S26 del G1F, Hapludol éntico serie Bolívar, Valores experimentales observados en la distribución de tamaño de partículas, masa acumulada en función del diámetro.

\subsubsection{Grupo 1 franco arenoso. Análisis grupal}

Este grupo lo integraron suelos provenientes de la Pampa Arenosa (S9, S10, S11, S12, S13, S14 y S31). La fracción dominante fue la arena con $61,7 \%$, le siguió el limo $26,6 \%$ y por último la arcilla representada con $11,8 \%$. La DTP del grupo en la escala sedimentológica (Figura 4.8) obtuvo una media de $\mathrm{Phi}=3,52$ equivalente a 86,9 um y un desvío estándar de 1,54 que lo clasifica como arena muy fina, pobremente seleccionada. 


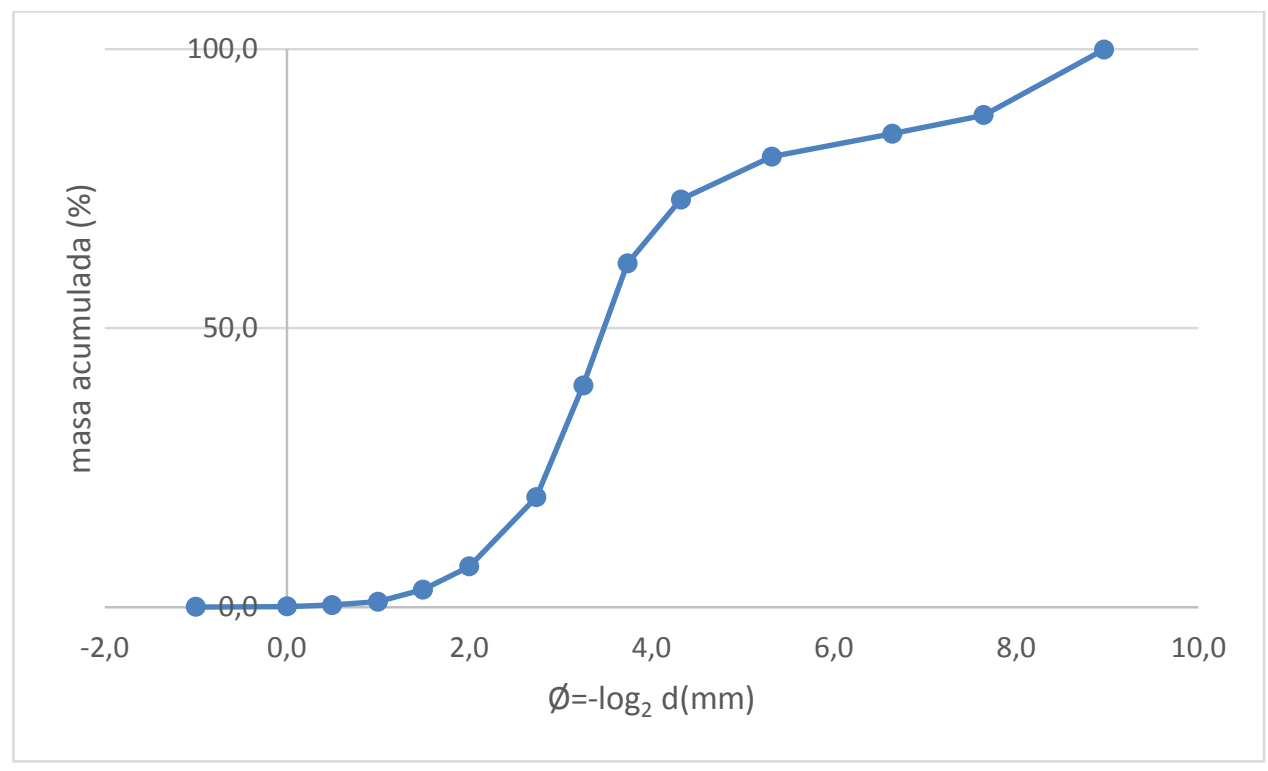

Figura 4.8. Distribución de tamaño de partículas de masa acumulada en función de Phi para el G1FA.

La DTP completa, de este grupo tampoco pudo ser ajustada por una sola función potencial, por lo que se resolvió dividir el rango de los valores experimentales en tres sectores aplicándose el modelo en cada uno de ellos (Figura 4.9).

En el primero, 2 a $44 \mu \mathrm{m}$, se describe prácticamente las arcillas más los limos y corresponde al $38 \%$ de la masa experimental, mientras que, en el segundo, 44 a $122 \mu \mathrm{m}$, se describe principalmente el dominio de arenas muy finas y algo de arenas finas. Entre los tramos uno y dos se alcanza a describir el $90 \%$ de la masa acumulada. El tercer tramo, 122 a $1000 \mu \mathrm{m}$, describe el 10\% restante de la masa acumulada, correspondiendo a la distribución acumulada de las arenas más gruesas, con $\mathrm{D}$ próxima a 3

Las expresiones del modelo, los parámetros del mismo y el ajuste para cada sector se presentan a continuación:

Primer sector:

$$
\begin{aligned}
& m 1 G 1 F A(\%)=8,557 \emptyset^{0,367} \quad \mathrm{D} 1=2,633 \\
& 2 \leq \varnothing(\mu \mathrm{m}) \leq 44 \\
& R^{2}=0,93 . p<0,001
\end{aligned}
$$


Segundo sector:

$$
\begin{gathered}
m 2 G 1 F A(\%)=0,774 \emptyset^{1,003} \quad \mathrm{D} 2=1,997 \\
44 \leq \emptyset(\mu \mathrm{m}) \leq 122 \\
R^{2}=0,76 . p<0,001
\end{gathered}
$$

Tercer sector:

$$
\begin{gathered}
m 3 G 1 F A(\%)=86,497 \emptyset^{0,022} \quad \text { D3 }=2,978 \\
122 \leq \varnothing(\mu \mathrm{m}) \leq 1000 \\
R^{2}=0,33 . p<0,001
\end{gathered}
$$

Incorporamos en este estudio nuevamente, la segunda dimensión fractal, puesto que el ajuste es elevado y abarca hasta el $90 \%$ la masa experimental descripta (Figura 4.9). En el tercer tramo del grupo, la $\mathrm{D}$ es próxima a 3 por lo tanto no tendría un comportamiento fractal.

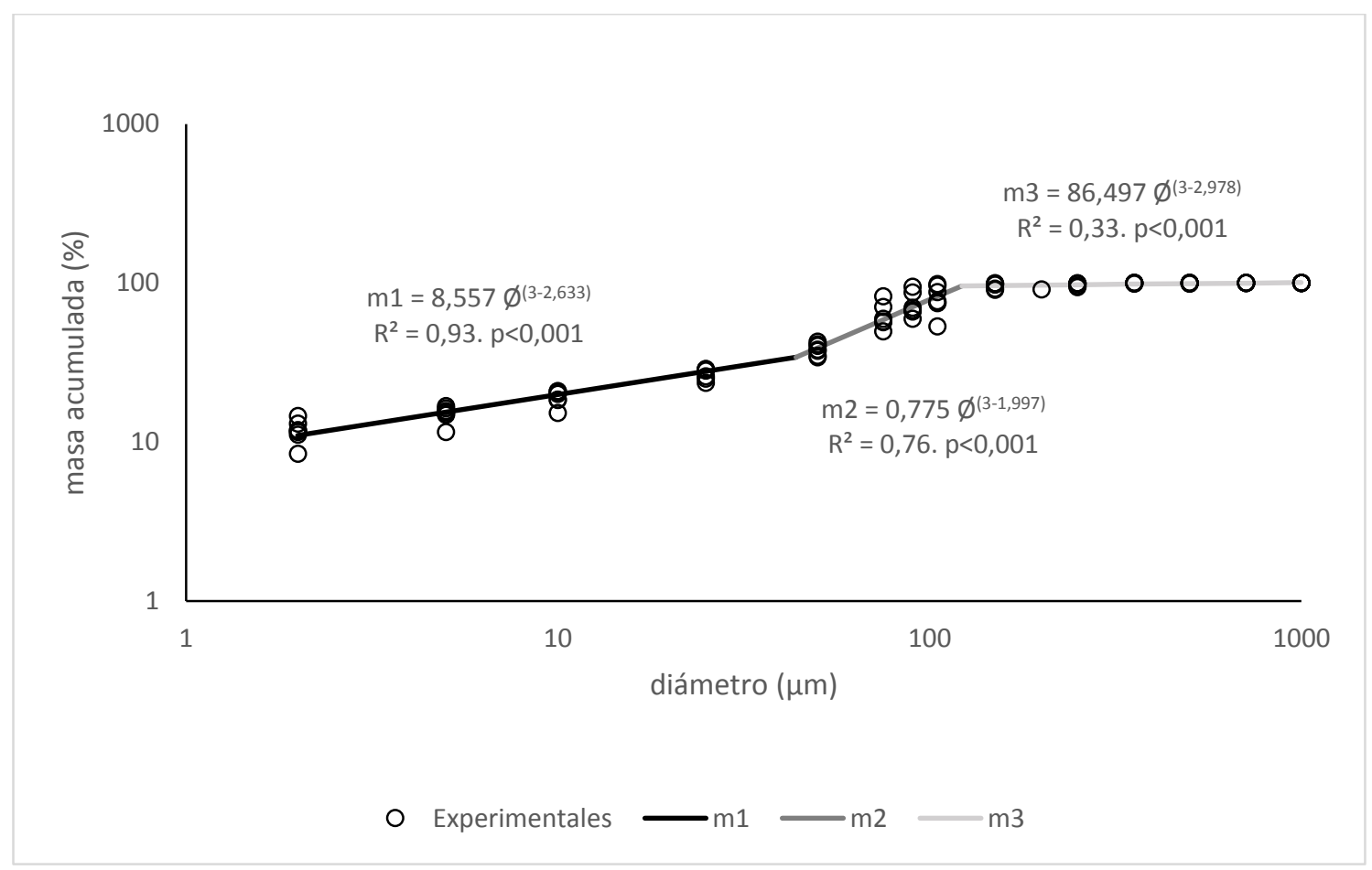

Figura 4.9. Modelo fractal para el G1FA, acotado por sectores, valores experimentales observados en la distribución de tamaño de partículas, masa acumulada en función del diámetro. 


\subsubsection{Ejemplo de análisis individual}

Se analizó el S9, de la serie Norumbega, en la escala sedimentológica obtuvo una media $\mathrm{Phi}=4,2$, equivalente a $56,3 \mu \mathrm{m}$ que lo califica como limo grueso pobremente seleccionado. Se encontraron experimentalmente hasta arenas gruesas pero la mayor proporción correspondió a arenas muy finas a finas.

El suelo presentó nuevamente tres sectores (Figura 4.10), se le aplicó el modelo a cada uno resultando los siguientes valores:

Primer sector:

$$
\begin{gathered}
m 159(\%)=8,872 \emptyset^{0,338} \quad \mathrm{D} 1=2,662 \\
2 \leq \emptyset(\mu \mathrm{m}) \leq 47,7 \\
R^{2}=0,99 . p<0,001
\end{gathered}
$$

Segundo sector:

$$
\begin{gathered}
m 2 S 9(\%)=0,292 \emptyset^{1,222} \quad \mathrm{D} 2=1,778 \\
47,7 \leq \emptyset(\mu \mathrm{m}) \leq 116,5 \\
R^{2}=1,0 . p<0,001
\end{gathered}
$$

Tercer sector:

$$
\begin{gathered}
m 3 S 9(\%)=92,342 \emptyset^{0,012} \quad \mathrm{D} 3=2,988 \\
116,5 \leq \emptyset(\mu \mathrm{m}) \leq 1000 \\
R^{2}=0,81 . p=0,01
\end{gathered}
$$

En el primer sector, m1S9, alcanza a describir el 34,0 \% de la masa y el límite es cercano al límite convencional de los limos, con un diámetro común de 47,7 $\mu \mathrm{m}$.

El segundo sector acumulado, entre 47,7 a $105 \mu \mathrm{m}$, alcanza hasta el $87,5 \%$ de la masa total, y el modelo propone llegar hasta $116 \mu \mathrm{m}$ incluyendo algo de arenas finas. El tercer sector, de las arenas de mayor tamaño, se corresponde con una distribución de partículas no fractales al tener la $\mathrm{D}$ próxima a 3.

Los ajustes individuales mejoran sustancialmente respecto al del grupo, como ocurrió en los suelos del G1F, los mismos pueden encontrase en: VinculosIG1FA CyD individuales.xlsx 


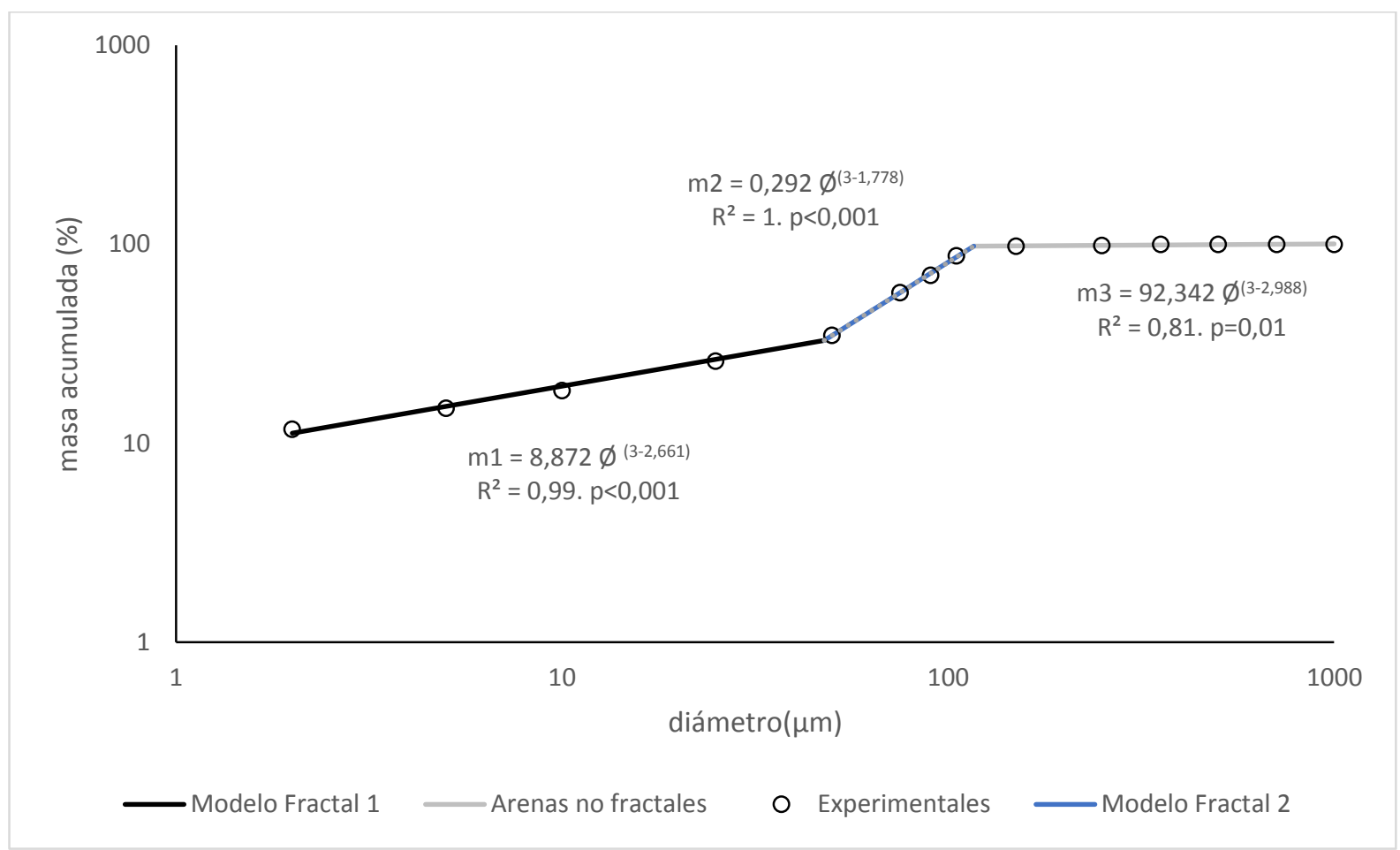

Figura 4.10. Modelo fractal acotado por sectores, ajustados particularmente al suelo S9, valores experimentales observados en la distribución de tamaño de partículas, masa acumulada en función del diámetro.

\subsection{Grupo 2}

Este grupo se formó por el S30 asociado a la serie Pirovano (Pv), un Udipsamente Tapto Mólico, franco gruesa, mixta, térmica. El grupo representa a los suelos más gruesos de textura areno franco de la Pradera Pampeana. La fracción arena es dominante con $80,6 \%$, le sigue el limo, $12,7 \%$ y la menos representada es la arcilla, con $6,7 \%$.

La DTP del grupo en la escala sedimentológica obtuvo un Phi medio= 1,95 equivalente a $259,2 \mu \mathrm{m}$ y fue clasificado como arena mediana, pobremente seleccionada.

La DTP de este grupo es diferente a las estudiadas en el grupo anterior.

La gráfica de masa acumulada en función del diámetro, ambos ejes en escala logarítmica, muestra dos sectores (Figura 4.11), uno curvo 2- $200 \mu \mathrm{m}$ y otro prácticamente horizontal desde los $200-1000 \mu \mathrm{m}$ para las arenas más gruesas. 


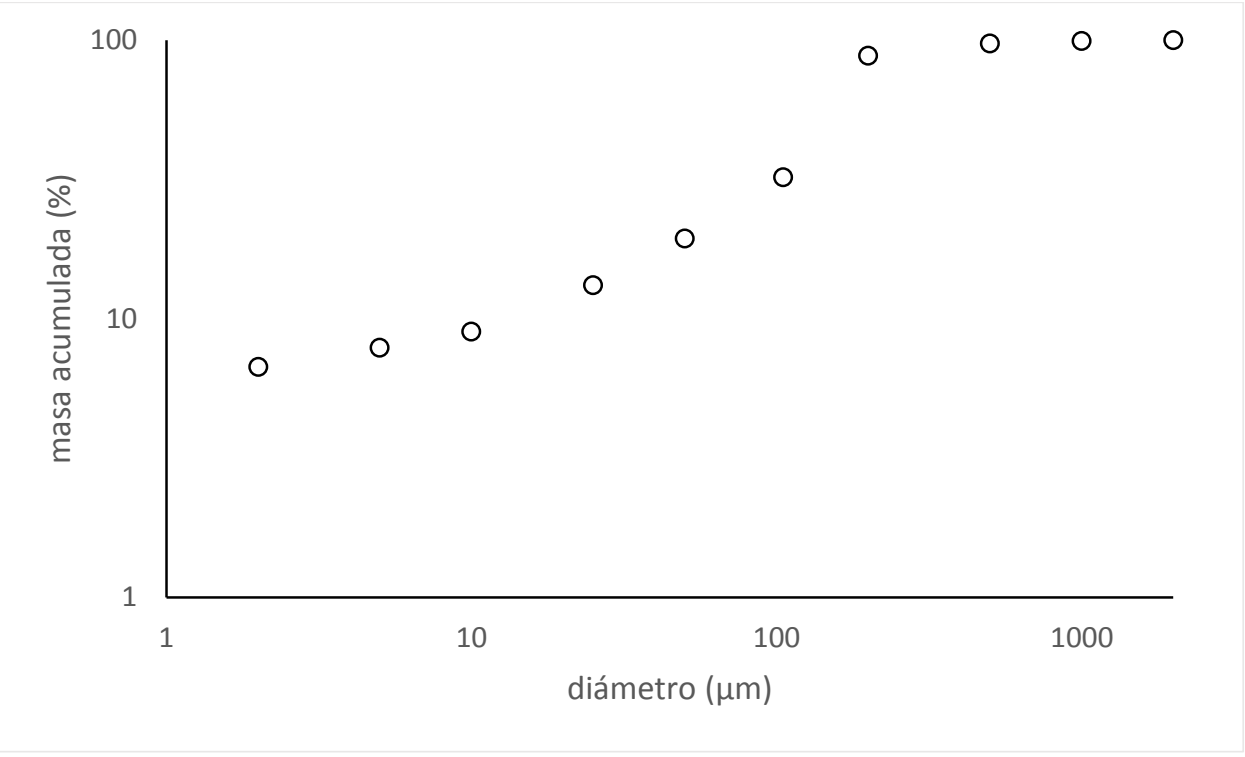

Figura 4.11. Distribución de tamaño de partículas para el G2, ambos ejes en escala logarítmica

El modelo potencial entre los diámetros 2 y $105 \mu \mathrm{m}$ no describió adecuadamente los datos experimentales. El sesgo en los residuos, (Figura 4.12), descarta su aplicación (Figura $4.14 b)$

Si ubicamos a este suelo en el triángulo textural, (Figura 4.1) los porcentajes de arena y arcilla lo posicionan a la derecha de la línea de Iso $\emptyset_{T}=2000 \mu \mathrm{m}$, área de suelos potencialmente no fractales, coincidiendo los resultados experimentales con el marco teórico.

De todos modos, se presenta a continuación el modelo fractal para los datos experimentales y el grado de ajuste hasta $105 \mu \mathrm{m}$ y se muestran en la Figura $4.14 \mathrm{~b}$.

El modelo obtenido resultó:

$$
\begin{aligned}
& m G 2=4,288 \emptyset^{0,393} \quad D=2,607 \\
& 2 \leq \emptyset(\mu \mathrm{m}) \leq 105 \\
& \mathrm{R}^{2}=0,94 . \mathrm{p}<0,0001
\end{aligned}
$$




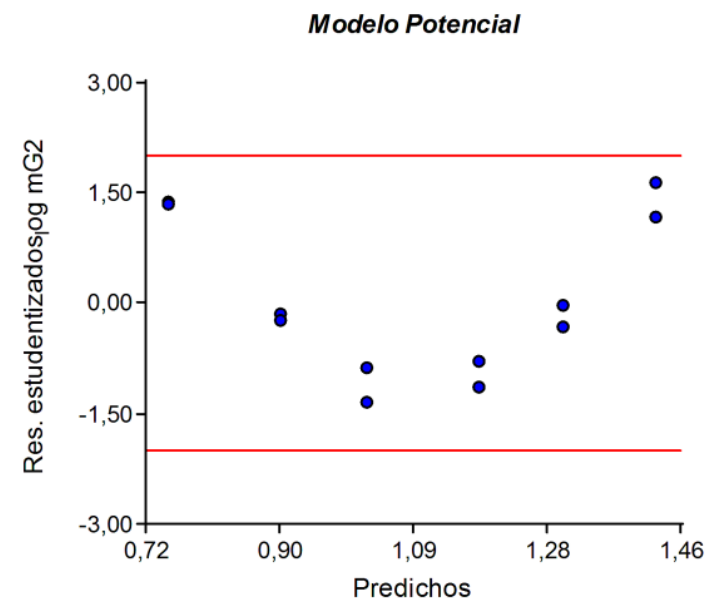

Figura 4.12. Residuos para el modelo fractal del G2

Los datos experimentales en este rango de diámetros fueron descriptos por un modelo lineal, que alcanzó a describir el 33,5\% de la masa experimental (Figura 4.13).

El modelo propuesto resultó:

$$
\begin{gathered}
m G 2(\%)=0,246 \emptyset+6,649 \\
2 \leq \emptyset(\mu \mathrm{m}) \leq 105 \\
R^{2}=1 . p<0,0001
\end{gathered}
$$

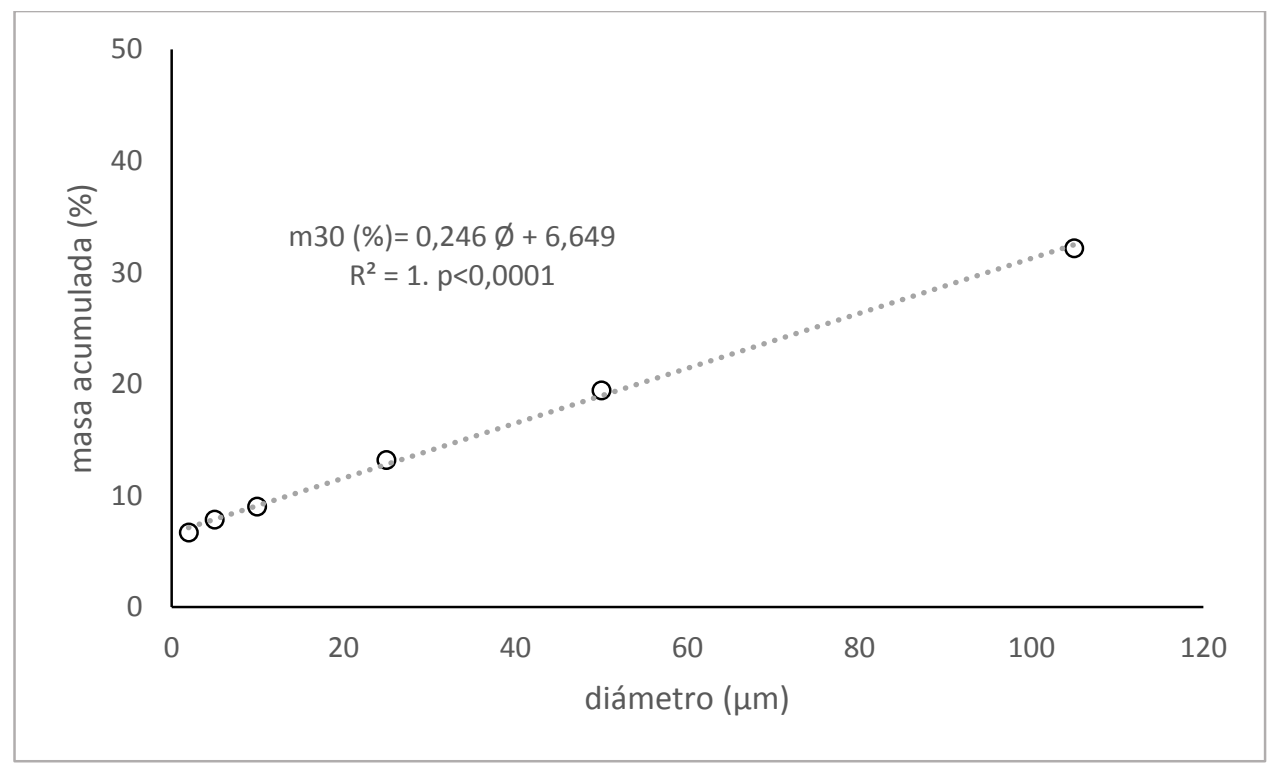

Figura 4.13. Modelo lineal propuesto para el G2 
La comparación entre el modelo lineal y el potencial pueden observarse en las Figuras 4.13a y b respectivamente.

G2, Modelo Lineal

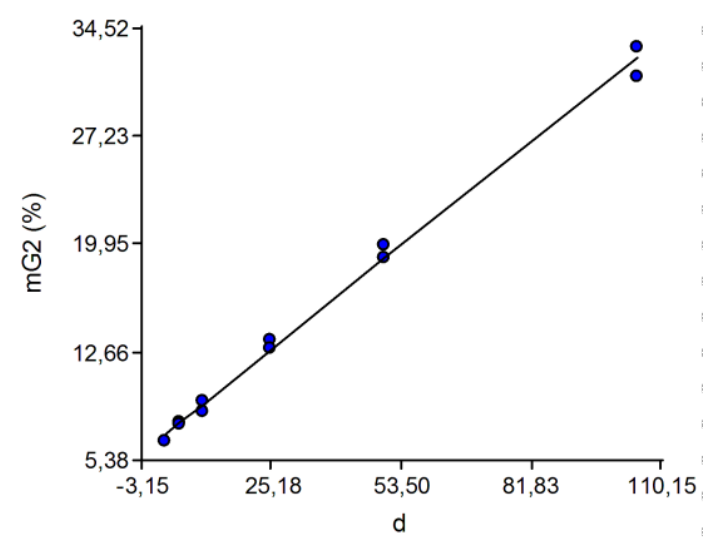

a

Figura 4.14.a. Modelo lineal propuesto para el G2. b Modelo Fractal, descartada para explicar la DTP en este Grupo de suelos

\subsection{Grupo 3. Análisis grupal}

Se formó con muestras de Argiudoles y Paleudoles, de la Pampa Ondulada, suelos profundos evolucionados a nivel de Familia, fina - muy fina, illitica y de régimen Térmico. La fracción dominante fue el limo con $66,7 \%$, le siguió la arcilla con $25,0 \%$ y por último la arena con $8,3 \%$. (Figura 3.1). En la escala sedimentológica fue calificado como limo mediano, pobremente seleccionado con una media en Phi 5,76, equivalente a 18,4 $\mu \mathrm{m}$ y un desvío estándar de 1,83.

La masa experimental acumulada hasta los $75 \mu \mathrm{m}$ fue de $98,4 \%$. Si bien se encontraron hasta "arenas finas" (105 - $250 \mu \mathrm{m}$ ), algunas eran de cuarzo y otras correspondían a fragmentos de concreciones de Fe-Mn, consecuencia de procesos de redoximórficos en pisos de horizontes A sobre horizontes argílicos potentes. La masa comprendida entre 75 y $250 \mu \mathrm{m}$ fue de 1,6\% y no aportó al modelo. El punto común entre ambos dominios, se obtuvo por la intercepción de las rectas de regresión, como en los casos anteriores.

El ØT de 67,4 $\mu \mathrm{m}$, calculado teóricamente, resultó próximo a los valores experimentales obtenidos.

La distribución de los datos experimentales de masa acumulada en función del diámetro, evidencia dos sectores (Figura 4.15). A continuación, se presentan los parámetros del modelo propuesto: 
Primer sector

$$
\begin{gathered}
m 1 \mathrm{G} 3(\%)=19,196 \emptyset^{0,392} \quad \mathrm{D} 1=2,608 \\
2 \leq \emptyset(\mu \mathrm{m}) \leq 67 \\
R^{2}=0,98 . p<0,0001
\end{gathered}
$$

Segundo sector

$$
\begin{gathered}
m 2 \mathrm{G} 3(\%)=96,828 \emptyset^{0,006} \mathrm{D} 2=2,994 \\
67 \leq \emptyset(\mu \mathrm{m}) \leq 250 \\
R^{2}=0,29 . p=0,0003
\end{gathered}
$$

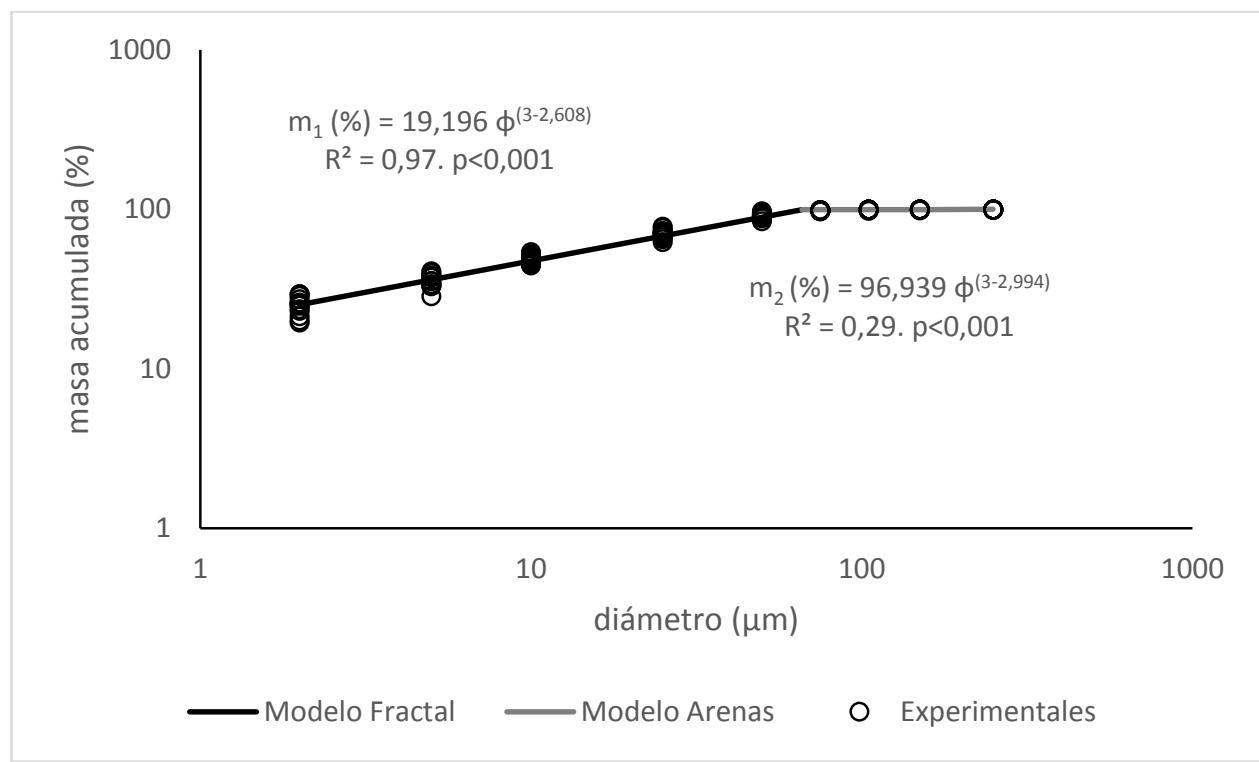

Figura 4.15. Modelo fractal para el G3, acotado por sectores, valores experimentales observados en la distribución de tamaño de partículas, masa acumulada en función del diámetro.

El valor de D2=2,994, próximo a 3 , indicaría un comportamiento euclidiano de la distribución, por lo tanto, no fractal.

\subsubsection{Ejemplo de análisis individual}

Desarrollamos el análisis sobre el S3 de la serie Portela. Como características este suelo tiene una clase textural franco limosa con $68,9 \%$ de limo, $25,4 \%$ de arcillas y $5,7 \%$ de arenas. La media en Phi fue de 5,82 es equivalente a $18 \mu \mathrm{m}$, calificando como limo mediano, pobremente 
seleccionado. El tamaño máximo de las arenas alcanzó hasta $250 \mu \mathrm{m}$, y experimentalmente a los $75 \mu \mathrm{m}$ se había acumulado el $99,3 \%$ de la masa total, Figura 4.16 .

Se encontraron dos zonas con diferentes comportamientos con una frontera en $\varnothing=63,3$ $\mu \mathrm{m}$. El modelo fractal quedó conformado por los siguientes parámetros:

Primer sector:

$$
\begin{gathered}
m 1 S 3(\%)=20,212 \emptyset^{0,384} \quad \mathrm{D} 1=2,616 \\
2 \leq \varnothing(\mu \mathrm{m}) \leq 63 \\
R^{2}=0,99 . p<0,001
\end{gathered}
$$

Segundo sector:

$$
\begin{gathered}
m 2 S 3(\%)=97,724 \emptyset^{0,003} \quad D 2=2,997 \\
63<\varnothing(\mu \mathrm{m})<250 \\
\mathrm{R}^{2}=0,78 . \mathrm{p}<0,046
\end{gathered}
$$

El ajuste de las arenas mayores a $75 \mu \mathrm{m}$, implica un valor experimental de la $\mathrm{D}=2,997$, muy próxima a 3 , que indicaría la característica euclidiana de esta fracción acumulada.

Como puede observarse, en Vinculos IG3 CyD individuales.xlsx para todos los suelos del Grupo, los ajustes individuales son elevados y las $D$ de las arenas próximas a 3 en todos los casos. 


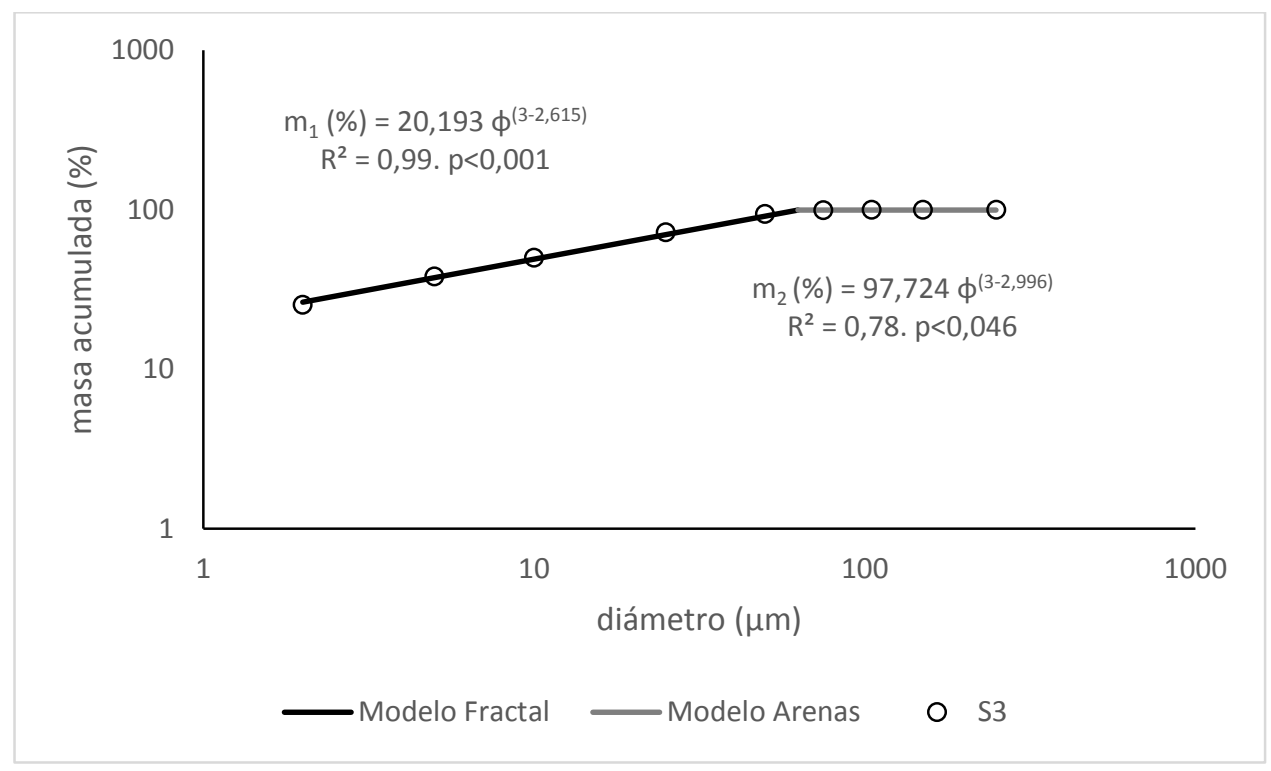

Figura 4.16. Modelo fractal acotado por sectores, ajustados particularmente al suelo S3. Valores experimentales observados en la distribución de tamaño de partículas, masa acumulada en función del diámetro.

\subsection{Análisis del modelo fractal de fragmentación experimental y teórico}

Se compararon las $\mathrm{D}$ experimentales, obtenidas de la curva de sedimentación (SMO) con las $D$ teóricas $\left(D_{T}\right)$ calculadas por la Ecuación 4.11. No se obtuvieron diferencias $(p=0,43)$. En el caso de los G1FA y G1F, se contrastaron las $D$ experimentales correspondientes al primer sector.

Se compararon las $C$ experimentales con las $C$ teóricas $\left(\mathrm{C}_{\mathrm{T}}\right)$, calculadas con la Ecuación 4.11. No se obtuvieron diferencias $(p=0,99)$. En el caso de los $G 1 F A$ y $G 1 F$, se contrastaron también las $\mathrm{C}$ experimentales correspondientes al primer sector. 


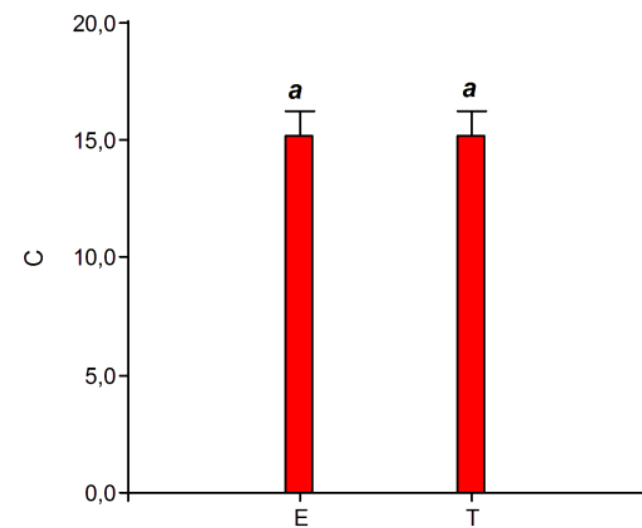

$\square \mathrm{c}$

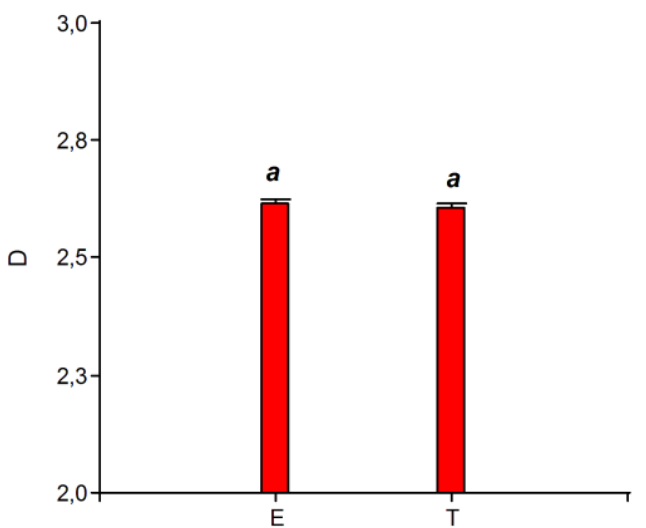

$\square \mathrm{D}$

Figura 4.17. Comparación entre valores experimentales " $E$ " obtenidos de las curvas de sedimentación y valores teóricos " $T$ " obtenidos con las Ecuaciones 4.10 y 4.11 para los parámetros C y $D$

Los resultados, posibilitan la estimación de los parámetros fractales en los suelos estudiados a partir del modelo teórico con los contenidos de arena y arcilla solamente. En el Capítulo 6, se presenta una exploración de la DTP a otros suelos argentinos mediante el modelo obtenido de esta manera.

\subsubsection{Comparación de los parámetros fractales entre Grupos}

Se analizaron los parámetros C y D entre los G1F-G1FA y G3, (Figura 4.18). Se encontró que la $D$ no tuvo diferencias significativas entre los grupos $(p=0,35)$, mientras que los $C$ fueron diferentes ( $p<0,0001$ ), el de menor valor para el G1FA, intermedio el G1F y el mayor para el G3. El resultado es consistente con el marco teórico, la $\mathrm{C}$ menor corresponde al grupo de suelos más gruesos. En el caso de los G1FA y G1F se compararon los $C$ y $D$ del primer sector. 

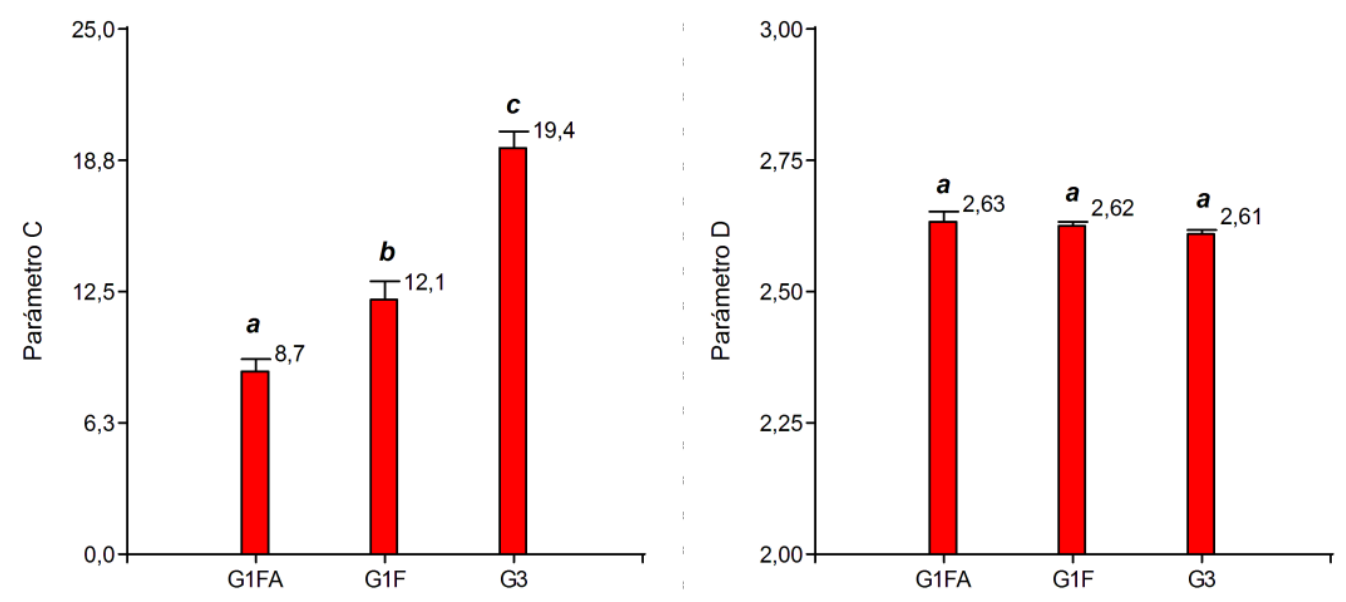

Figura 4.18. Comparación de los parámetros fractales $C$ y $D$ por grupo. Medias con una letra común no son significativamente diferentes $(p>0,05)$

Iriondo $(1990,1992)$ e Iriondo y Kröhling $(1995,1996)$ distinguen dentro del que denominan "Sistema eólico pampeano" el 'Mar de arena' hacia el centro-oeste y una faja periférica de "loess" hacia el este. Esta zonación sería consecuencia de un proceso de selección granulométrica como consecuencia del transporte eólico desde las fuentes de aporte, con una sedimentación proximal de arena y una distal de limo. Es así que el material parental de los suelos grada desde limos loéssicos en el este hasta arenas limosas en el oeste (Gonzalez Bonorino, 1966). El modelo fractal captó estas diferencias granulométricas producto de la sedimentación diferencial, con el parámetro "C". Al no obtenerse diferencias entre las D, los suelos tienen igual escalamiento y podría asumirse que corresponden a un mismo material original. Este resultado obtenido con el modelo fractal sobre el material original, es consistente con estudios geológicos y geomorfológicos de la pradera pampeana.

\subsubsection{Síntesis sobre el comportamiento fractal de las distribuciones de tamaño de} partículas, sin materia orgánica

En el Grupo 3, formado por suelos franco limosos, el modelo fractal de fragmentación describió adecuadamente a estos suelos de la pampa ondulada hasta la fracción de arenas 75 $\mu \mathrm{m}$ con valores de $\mathrm{D}$ medio de 2,608 variando entre 2,540 - 2,659 y los de $C$ medios 19,369 variando entre $14,723-23,313$. La fracción de partículas de arenas $(75-250 \mu \mathrm{m})$ con D próxima a tres, evidenciaron una naturaleza no fractal. Las masas correspondientes a esta fracción, no fueron incluidas para los cálculos, son escasas y podrían responder a un material original de origen fluvial aportado por el río Paraná, diferente al Loess. (Morras, 2003).

Los suelos asociados al Grupo 1 franco y franco arenoso, presentan déficit de partículas en los tamaños arcilla, limos y parte de las arenas muy finas. El G1F, obtuvo para el primer sector una D media de 2,624 variando entre 2,590 - 2,642 y C media 12,088 variando 8,716 - 14,299. 
Para el segundo sector, la D media fue 2,442 variando entre 2,357 - 2,493 y C media de 6,494 variando entre $3,873-8,533$.

EI G1FA obtuvo para el primer sector una D media de 2,633 variando $2,542-2,669$ y C media 8,683 variando $5,707-10,375$. Para el segundo sector la $D$ media fue 1,931 variando1,773 $-2,214$.

Con estas características la curva de masa acumulada en función del diámetro es de tipo sigmoidea y es necesario recurrir a dos modelos fractales para describir ambos sectores. Entre ambos modelos se puede explicar hasta el $90-95 \%$ de la masa acumulada. El tercer sector, presenta características euclidianas.

El Grupo 3, formado por suelos franco limosos, el modelo fractal de fragmentación describió adecuadamente a estos suelos de la pampa ondulada hasta la fracción de arenas de $75 \mu \mathrm{m}$. La fracción de partículas de arenas $(75-250 \mu \mathrm{m})$ con D próxima a tres, evidenciaron una naturaleza no fractal. Las masas correspondientes a esta fracción, no fueron incluidas para los cálculos, son escasas y podrían responder, como se mencionó a un material original de origen fluvial aportado por el río Paraná, diferente al Loess (Morras, 2003).

Los suelos asociados al Grupo 1 franco arenoso y franco, presentan déficit de partículas en los tamaños arcilla, limos y parte de las arenas muy finas. Con estas características la curva de masa acumulada en función del diámetro es de tipo sigmoidea y es necesario recurrir a dos modelos fractales para describir ambos sectores. Entre ambos modelos se puede explicar hasta el $90-95 \%$ de la masa acumulada. El tercer sector, presenta características euclidianas.

El Grupo 2, representa a los suelos residualmente más gruesos de la Pradera Pampeana y la distribución de tamaño de partículas no es descripta por un modelo fractal.

\subsection{Discusión del modelo fractal sin materia orgánica}

En base a la propuesta de varios autores Tyler y Wheatcraft (1992); Bitelli (1999); Bezhad y Daigle (2015), se aplicó el método de acotar e indicar los límites del modelo y los resultados fueron satisfactorios, mejorando el ajuste y el alcance en la masa descripta. Acotar el alcance del modelo, de alguna manera contradice los trabajos realizados por Stanchi et al. (2008).

Tyler y Wheatcraft (1992), manifestaron la fuerte relación entre $D$ y el contenido de arcilla, para estos autores este comportamiento era prometedor, aunque existirían dificultades con suelos que exhiben solo rangos limitados de comportamiento fractal. Bitelli et al. (1999), Filgueira et al. (2007) representaron las $D$ en función del porcentaje de arcilla, pero sin indicar el tipo de función. Gunal et al. (2011), proponen un polinomio de segundo grado, que podría describir estadísticamente la curva. Este resultado sería incorrecto, puesto que la relación es de tipo logarítmico y debería asegurarse que los $\emptyset_{T}$ de las muestras sean homogéneos. 
En la Figura 4.19 se graficaron las $D$ en función de la arcilla \% y también las funciones teóricas para $\emptyset_{T}$ de 60, 100 y $1000 \mu \mathrm{m}$, puede observarse que en los suelos de diferentes Grupos ocurren superposiciones: para igual porcentaje de arcilla, D diferente. Este resultado puede explicarse mediante el valor del $\emptyset_{T}$, contemplado en el valor de $C$. Los suelos del G3, tienen un $\mathrm{C}=19,4$ y les corresponde un promedio de $\varnothing_{T}=67,3 \mu \mathrm{m}$. Los suelos del $\mathrm{G} 1 \mathrm{~F}$ tienen un $\mathrm{C}=12,1 \mathrm{y}$ les corresponde un promedio de $\emptyset_{T}=259,8 \mu \mathrm{m}$, mientras que los suelos del G1FA con un $C=8,7$, les corresponde en promedio un $\emptyset_{T}=792,5 \mu \mathrm{m}$. El parámetro "C" no estaba descripto en los trabajos sobre fractales, en los cuales solo se considera la dimensión fractal " $D$ ".

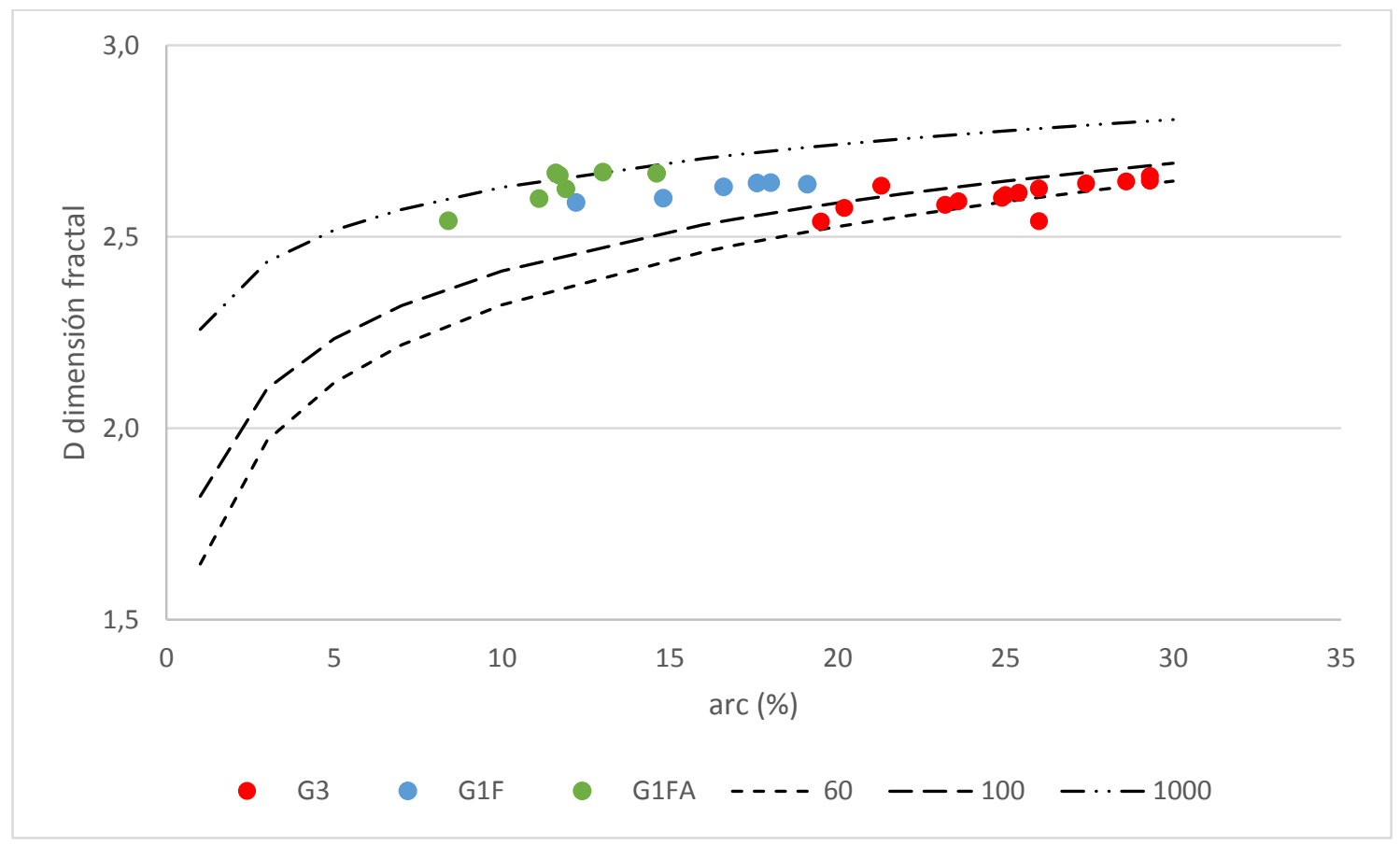

Figura 4.19. Valores de D experimental en función del contenido de arcilla. Se representaron como referencia en líneas punteadas las curvas teóricas correspondientes a D para $\emptyset_{T}(\mu \mathrm{m})=60 ; 100$ y 1000 .

Puesto que los Grupos no difieren en la dimensión fractal de fragmentación $(p=0,35)$, el parámetro $C$ sería una función lineal del contenido de arcillas (Ecuación 4.11). Se verificó dicha relación con los valores experimentales de $\mathrm{C}$ y porcentaje de arcilla, para todos los suelos estudiados. (Figura 4.20a) 

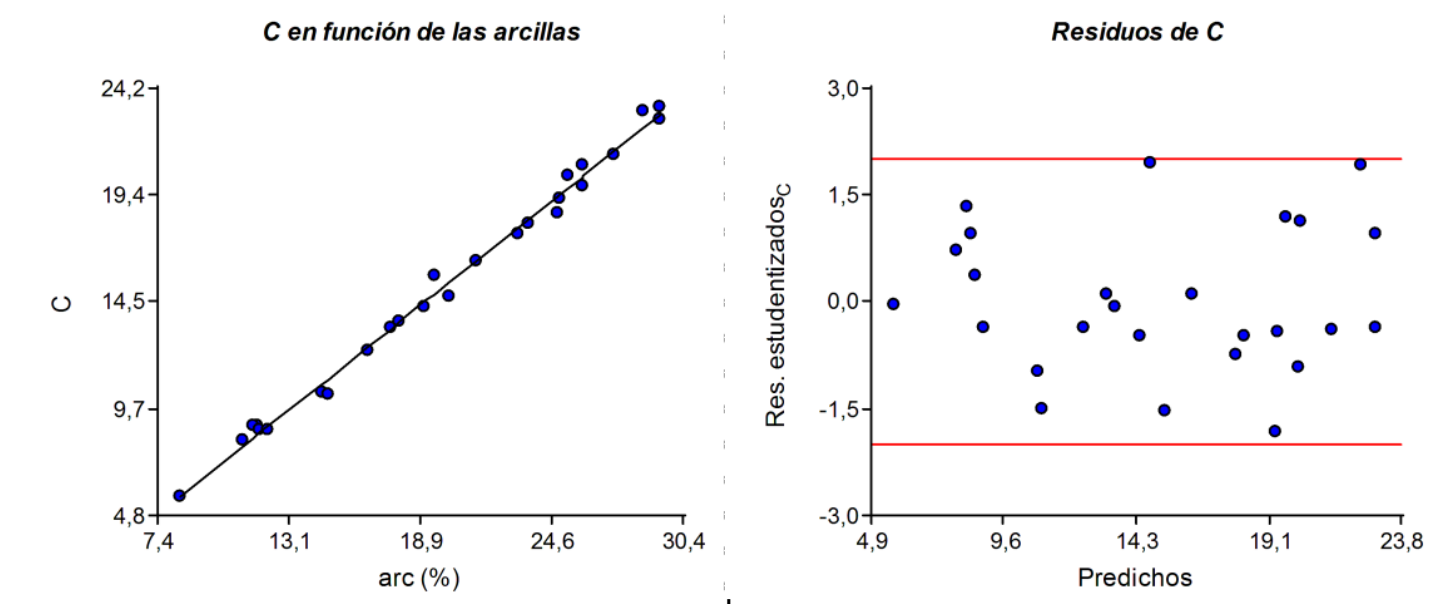

a b

Figura 4.20. a Relación lineal de "C" en función del contenido de arcillas. b Residuos del modelo lineal propuesto.

Bartoli et al. (1991) confirmaron que los suelos de textura fina son bien caracterizados por una distribución fractal, mientras no sucedía lo mismo con los cuarzo-arenosos, Barak et al. (1996), concluyeron que la distribución log-normal mostró mejor ajuste que la distribución fractal para la fracción gruesa. Avnir et al. (1985) demostraron que el cuarzo puro y los suelos cuarzo arenosos revelan valores de dimensión fractal relativamente bajos.

Se encontró que una porción de las arenas, las muy finas a finas fueron descriptas adecuadamente por el modelo fractal. En el caso del G3, con un solo modelo y en los G1F y G1FA, empleando dos modelos. En los Grupos se observó que las fracciones mayores a $120 \mu \mathrm{m}$ no fueron fractales con $D$ próxima o igual a 3.

Podríamos resumir lo expuesto por Avnir et al. (1985), Bartoli et al. (1991) y Barak et al. (1996) que la fracción más gruesa del suelo, las arenas, tienen dimensiones fractales pequeñas o son descriptas por modelos diferentes al fractal. Bitelli et al. (1999), planteó definir los límites de las fracciones acilla, limo y arena de acuerdo con el comportamiento de la DTP en función del diámetro.

Este enfoque permite asociar a las arenas muy finas con los limos como en el G3 o considerar la inflexión como en el G1F y G1FA. Se trabajó con los limites propuestos por USDA y expresar los resultados con esta escala. Se encontró que diferentes porciones de arenas todas muy finas y partes de las finas pueden ser descriptas por el modelo fractal, mientras que los tamaños superiores no serían descriptos por el modelo en estos suelos Pampeanos. 


\subsection{Conclusiones}

Los suelos de la Pampa Ondulada, franco-limosos y franco arcillo limoso la distribución de tamaño de partículas acumulada es de tipo fractal.

Los suelos, que necesitan de dos modelos para explicar hasta el $95 \%$ de la distribución de tamaño de partículas acumulada, la fractalidad es intermedia.

Los suelos areno franco o más gruesos, la distribución de tamaño de partículas acumulada, no es de tipo fractal. 


\section{Resultados: el modelo fractal y su aplicación al análisis de la distribución de tamaño de microagregados}

Capítulo 5 


\section{$5 \quad$ Estudio fractal de la distribución de tamaño de microagregados}

\subsection{Resultados de las sedimentaciones con materia orgánica}

Se analizan mediante el modelo fractal las sedimentaciones CMO. Cuando hablamos de las fracciones arena limo y arcilla, nos referiremos a la mezcla de partículas y pseudopartículas que se corresponden con los tamaños aparentes respectivos.

El análisis de los residuos mostró similar comportamiento que en el tratamiento SMO.

\subsection{Grupo 1. CMO. Análisis grupal}

El G1F y G1FA, al estudiarlo CMO obtuvo bajos CV (\%) para las arenas y el Phi medio de la DTM, siendo de $4,4 \%$ y $12,7 \%$, respectivamente. Las gráficas de masa acumulada en función de Phi, resultaron próximas y se los analizó como grupo único, G1CMO (Figura 5.1a). De la unificación de ambos, surge la clase textural aparente (Figura 5.1b) y la distribución de partículas en función de Phi (Figura 5.1c).
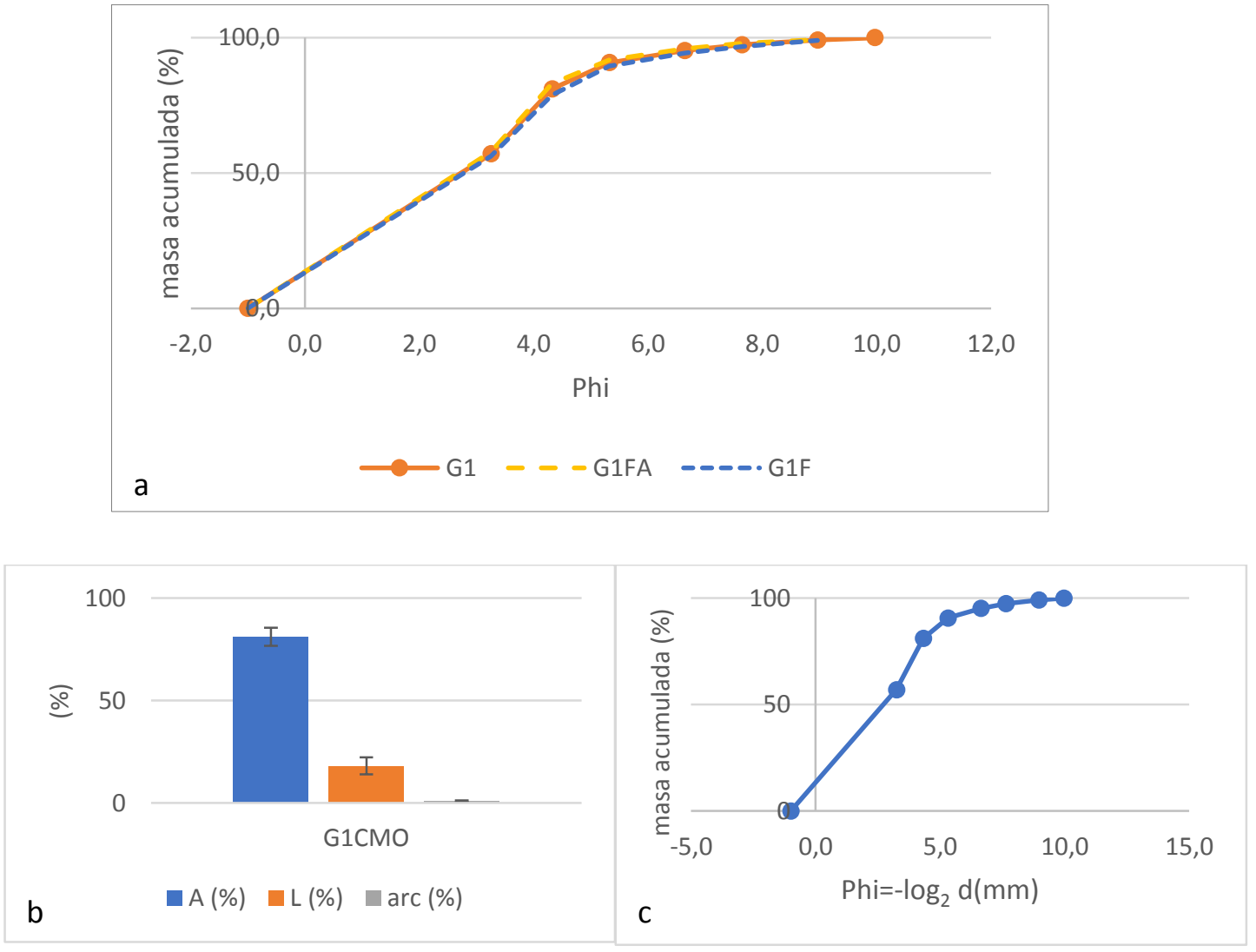

Figura 5.1a. Curva comparativa de masa acumulada en función de Phi para G1FA-G1 y G1F. b fracciones texturales aparentes para el G1 y c curva de masa acumulada en función de Phi para el G1. 
La DTM, de masa en función del diámetro, se analizó mediante un solo modelo fractal hasta $100 \mu \mathrm{m}$, describiendo el $39,1 \%$ de la masa experimental. El resto del material, 60,9\%, corresponde a microagregados de mayor tamaño. El $\emptyset_{T}$ teórico, resultó de 258,9 $\mu \mathrm{m}$. En la Figura $5.2 a$ se observa la distribución de residuos y en la Figura 5.2b los valores experimentales y el modelo propuesto.

El modelo fractal obtenido:

$$
\begin{gathered}
m 1 G 1(\%)=0,573 \emptyset^{0,929} \quad \mathrm{D} 1=2,071 \\
2 \leq \emptyset(\mu \mathrm{m}) \leq 100 \\
\mathrm{R}^{2}=0,94 . p<0,0001
\end{gathered}
$$

Los parámetros del modelo, fueron menores a los del pretratamiento SMO. Los resultados, son coherentes con el marco teórico, por tratarse de una suspensión de microagregados.

G1 CMO

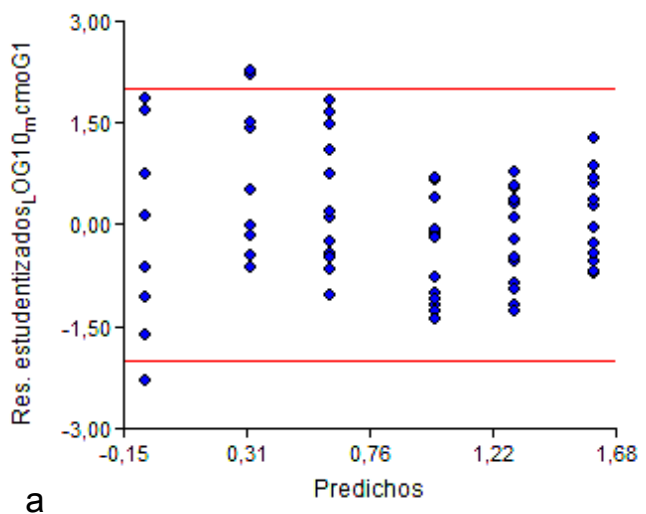

G1 CMO

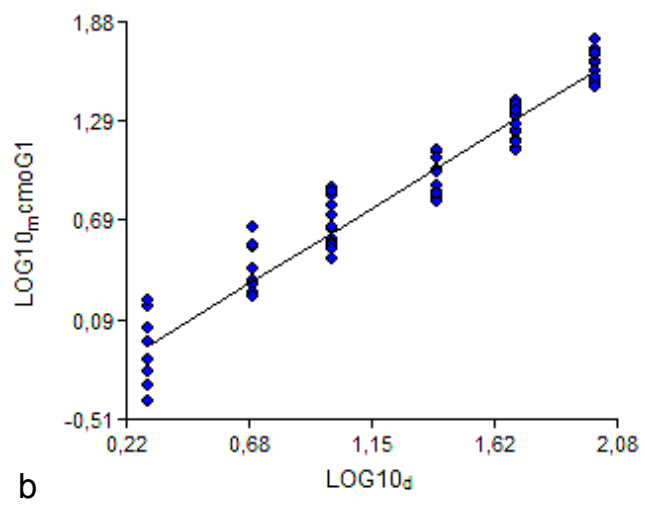

Figura 5.2a. Distribución de los residuos para el modelo. b. Datos experimentales y recta de regresión ajustada (CMO).

\subsubsection{Ejemplo de análisis individual}

Se analizó la muestra del S24, asociado a la serie Bolívar, un Hapludol Éntico Franca gruesa mixta térmica. Del análisis SMO, se obtiene una clase textural franco arenoso con un valor medio de partículas, expresado en Phi $=4,2$, equivalente a $56,3 \mu \mathrm{m}$ que lo calificó como limo grueso. El contenido de COT fue de $10,1 \mathrm{~g} \mathrm{~kg}^{-1}$ y MOT, $17,4 \mathrm{~g} \mathrm{~kg}^{-1}$. El análisis CMO, le otorgó una clase textural aparente areno franco (Figura 5.3a) y en la escala sedimentológica (Figura 5.3b) un valor de la media $\mathrm{Phi}=2,18$ equivalente a 219,9 $\mu \mathrm{m}$ con un desvío estándar = 2,03 que lo calificó como "arena fina, muy pobremente seleccionada". 


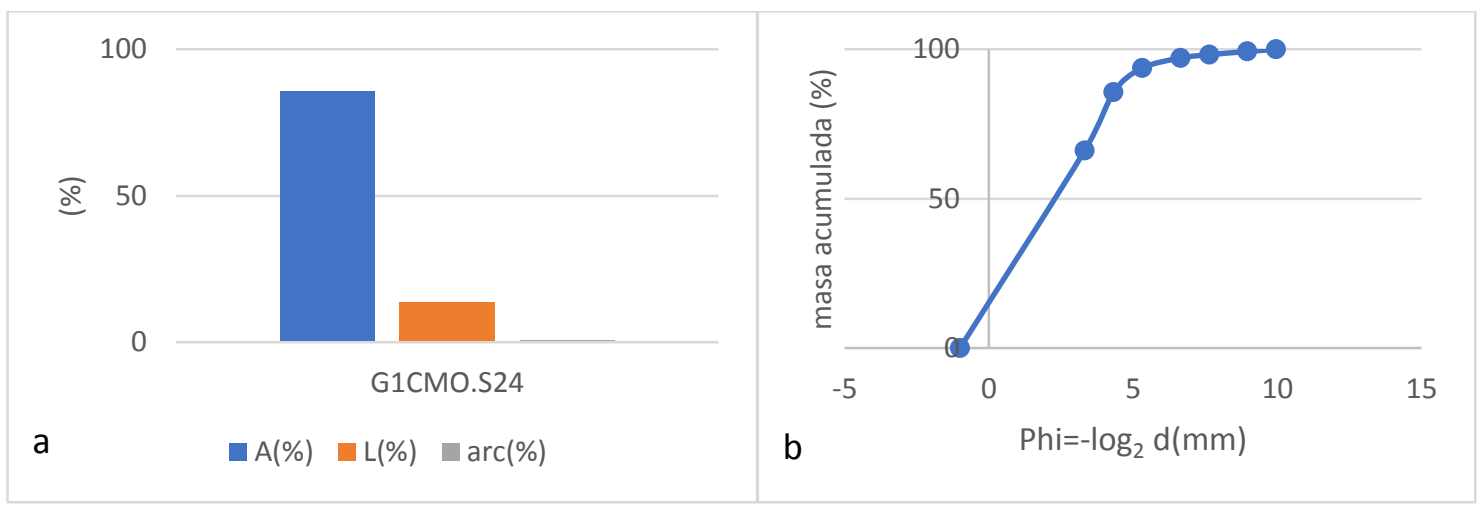

Figura 5.3a. Proporciones de arena limo y arcillas aparentes para el S24CMO. b Curva de masa acumulada en función de Phi para el S24.

La DTM tuvo un comportamiento que puede asimilar con el fractal, hasta los $100 \mu \mathrm{m}$, incluyendo el $33,9 \%$ de la masa experimental (Figura 5.4).

El modelo resultante obtenido:

$$
\begin{aligned}
\operatorname{mS} 24 c m o(\%) & =0,344 \emptyset^{0,962} \quad \mathrm{D} 1=2,038 \\
2 & \leq \varnothing(\mu \mathrm{m}) \leq 100 \\
R^{2} & =0,99 . p<0,001
\end{aligned}
$$

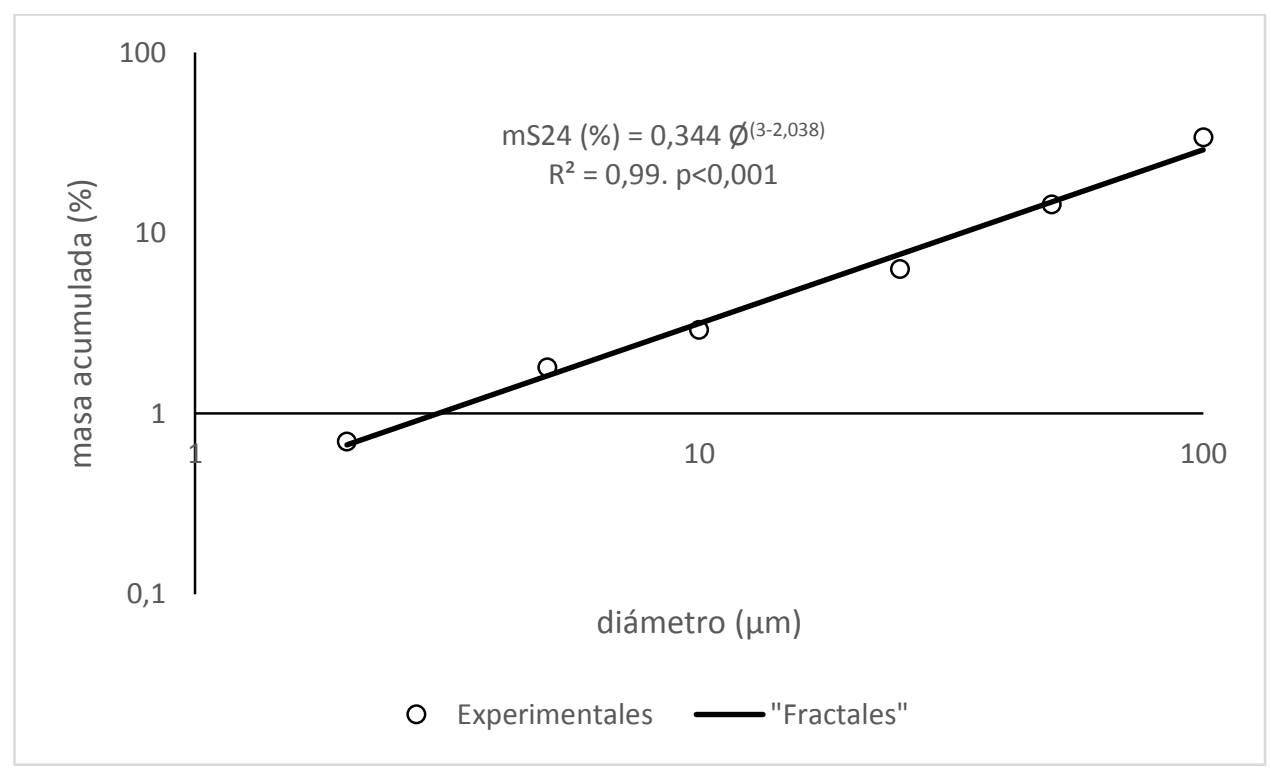

Figura 5.4. Distribución de tamaño de microagregados (CMO) en función del diámetro $\varnothing(\mu m)$. 
En el estudio individual, el ajuste del modelo evaluado por el coeficiente de determinación $\mathrm{R}^{2}$ mejoró en todos los casos, como puede observarse en Vinculos $\mid \mathrm{G} 1 \mathrm{CMO}$ CyD individuales.xlsx

Se observa, de la misma manera que en el G1CMO, el modelo describe hasta el $33,4 \%$ de la masa experimental. Los valores de $C$ y $D$ respecto al tratamiento SMO también fueron menores.

\subsection{Grupo 2. CMO}

Este Grupo, está formado por la muestra del suelo 30, asociado a la serie Pirovano. La sedimentación del suelo $\mathrm{CMO}$, presentó un comportamiento no fractal como en el caso del pretratamiento SMO y la distribución de tamaño de microagregados fue descripta por un modelo lineal. El mismo describió el 6,9\% de la masa experimental con elevado ajuste hasta un diámetro aparente de $50 \mu \mathrm{m}$. El valor experimental correspondiente a $100 \mu \mathrm{m}$ quedó afuera del modelo de regresión empleando (Figura 5.5). En este Grupo se aprecia la elevada cantidad de partículas y microagregados con diámetro aparente mayor a $100 \mu \mathrm{m}$.

El modelo propuesto resultó:

$$
\begin{gathered}
m G 2(\%)=0,137 \emptyset-0,228 \\
2 \leq \emptyset(\mu m) \leq 50 \\
R^{2}=0,99 . p<0,001
\end{gathered}
$$




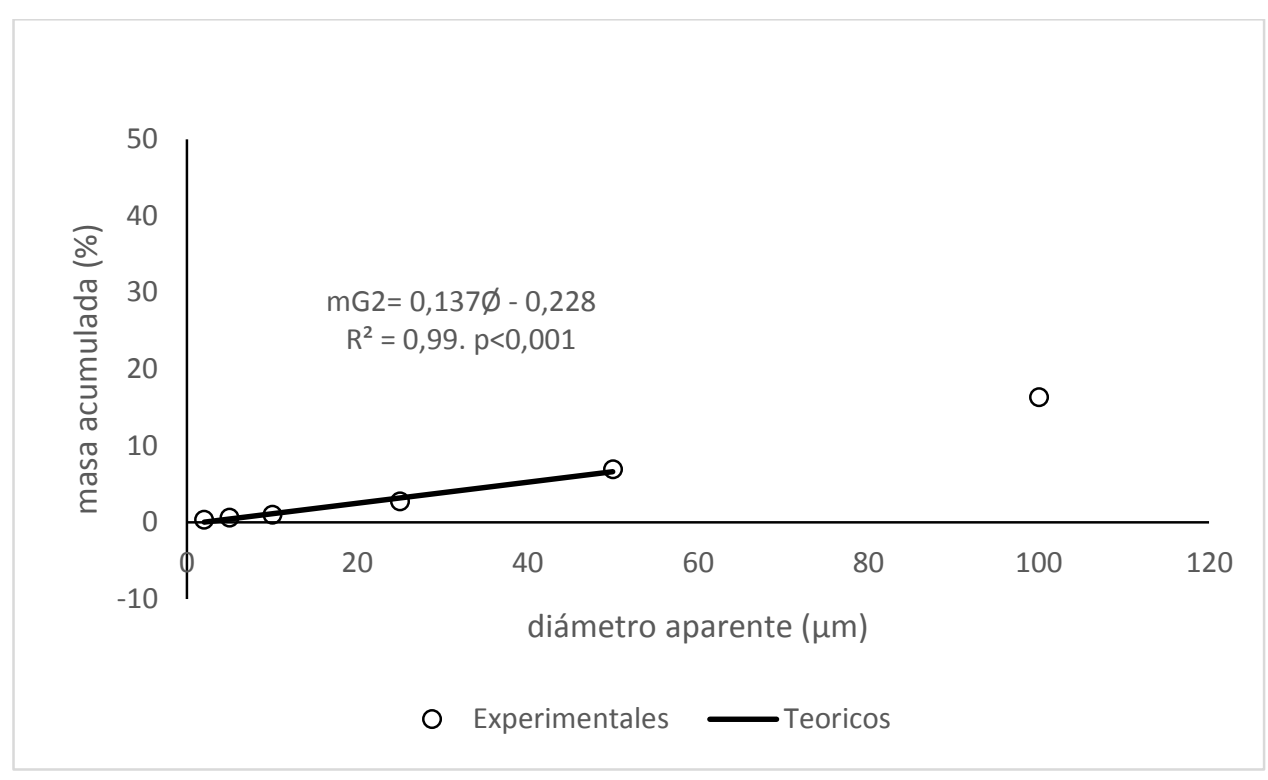

Figura 5.5 Distribución de tamaño de microagregados (CMO) en función del diámetro $\varnothing(\mu m$, para el G2.

\subsection{Grupo 3. CMO. Análisis grupal}

La DTM, de masa en función del diámetro para el G3CMO, se analizó mediante un solo modelo fractal hasta los $100 \mu \mathrm{m}$, alcanzando a describir el 69,1\% de la masa experimental (Figura 5.6). El resto del material, 39,9\%, correspondió a microagregados de mayor tamaño. El $\emptyset_{T}$ teórico, resultó igual a 157,4 $\mu \mathrm{m}$.

El modelo fractal obtenido:

$$
\begin{aligned}
& \operatorname{G3cmo}(\%)=1,678 \emptyset^{0,808} \quad \mathrm{D} 1=2,192 \\
& 2 \leq \emptyset(\mu \mathrm{m}) \leq 100 \\
& R^{2}=0,94 . p<0,001
\end{aligned}
$$

Los valores de $\mathrm{C}$ y $\mathrm{D}$ obtenidos fueron menores a los del tratamiento SMO, similar a lo ocurrido en los Grupos 1 y 2 CMO. 


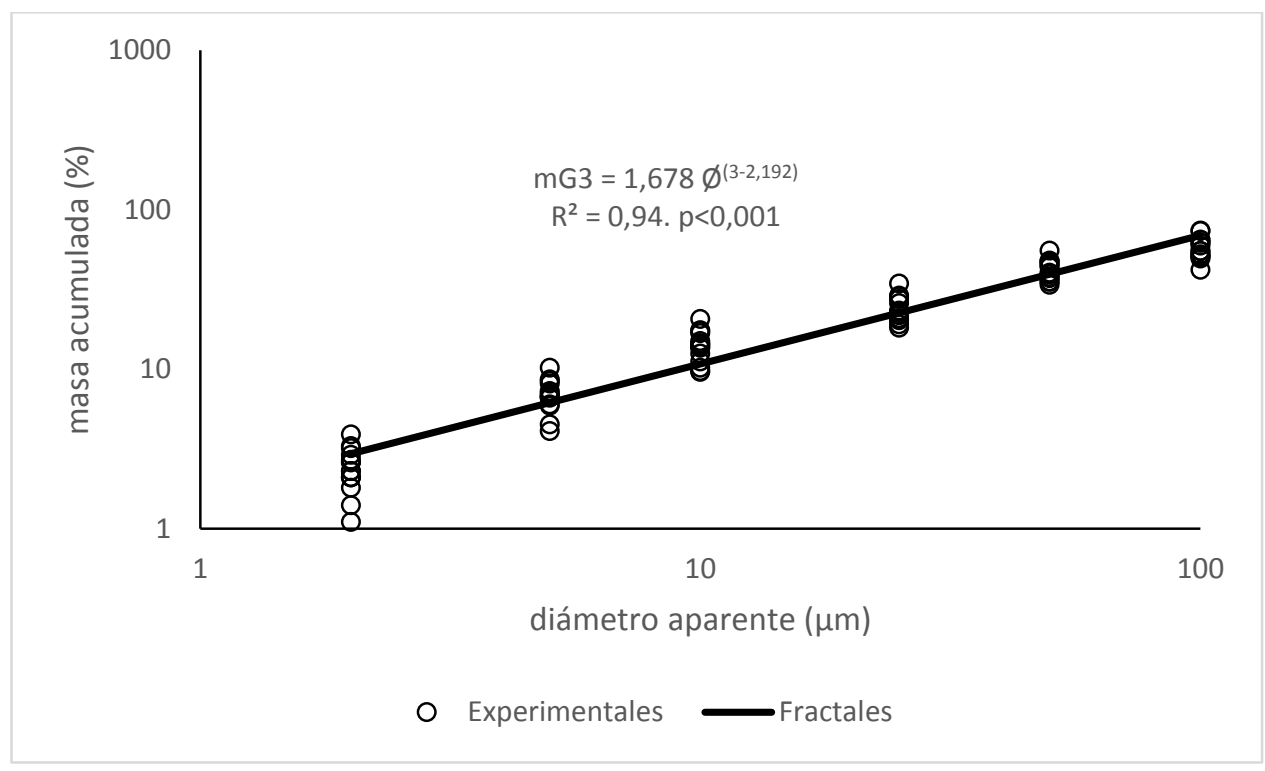

Figura 5.6. Distribución de tamaño de microagregados (CMO), para el G3, en función del diámetro $\varnothing(\mu m)$. Modelo fractal propuesto

\subsubsection{Ejemplo de análisis individual}

Se presenta el análisis de la muestra del suelo 20, asociado a la serie Magdalena extraído del Partido homónimo, un Paleudol Vértico Muy Fina, Illítica, térmica (USDA Soil Taxonomy 2010). La clase textural fue franco limoso, con $8 \%$ de arena, $66 \%$ de limo y $26 \%$ de arcilla. En la escala sedimentológica obtuvo una media en Phi $=5,84$, equivalente a $17,5 \mu \mathrm{m}$ y un desvío estándar de 1,84 que lo clasificó como "limo mediano, pobremente seleccionada".

Del análisis de la DTM, obtuvo una clase textural aparente "franco arenoso" se observa en la Figura 5.7 a y en la escala sedimentológica (Figura 5.7b) se obtuvo una media de Phi=3,3, equivalente a 101,7 $\mu \mathrm{m}$ y un desvío estándar de 2,67 que lo clasificó como "arena muy fina, muy pobremente seleccionada". 


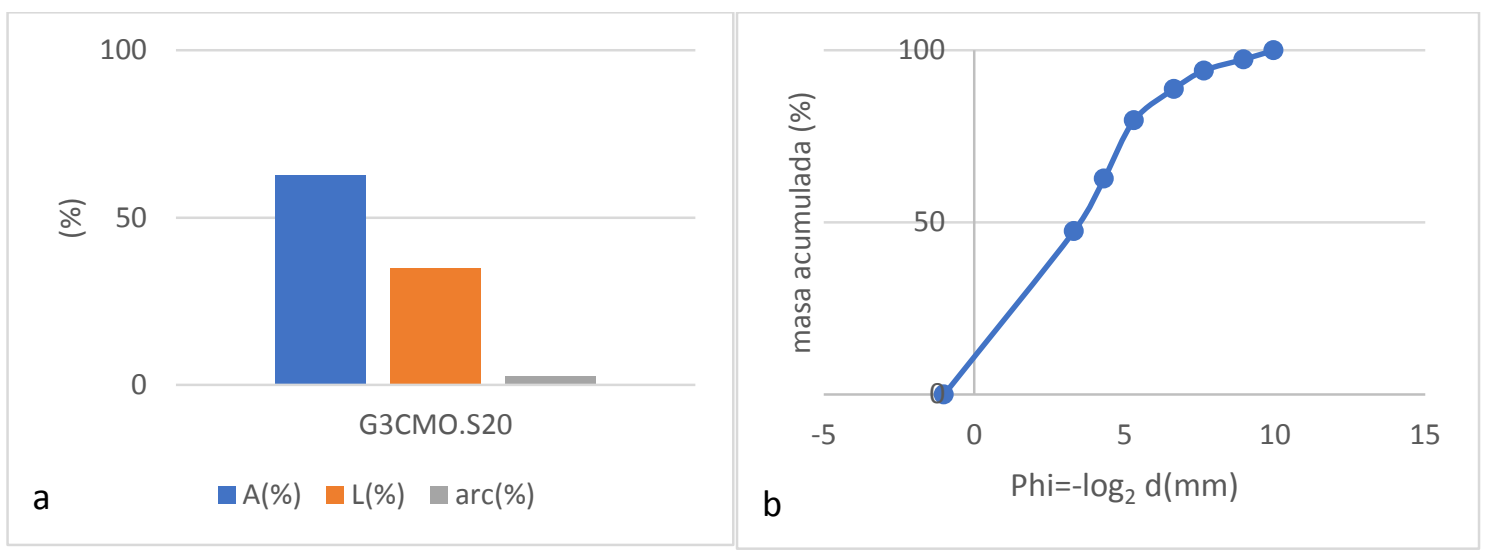

Figura 5.7a. Proporciones de arena limo y arcillas aparentes para el G3CMO. b Curva de masa acumulada en función de Phi para el mismo Grupo.

El modelo fractal se ajustó a los datos experimentales hasta $100 \mu \mathrm{m}$ de diámetro aparente, describiendo el $52,5 \%$ de la masa acumulada, el resto del material, corresponde a microagregados de mayor tamaño (Figura 5.8).

El suelo modelado resultó con los siguientes parámetros:

$$
\begin{aligned}
& m S 20 c m o(\%)=1,674 \emptyset^{0,774} \quad \mathrm{D} 1=2,226 \\
& 2 \leq \emptyset(\mu \mathrm{m}) \leq 100 \\
& R^{2}=0,99 . p<0,001
\end{aligned}
$$

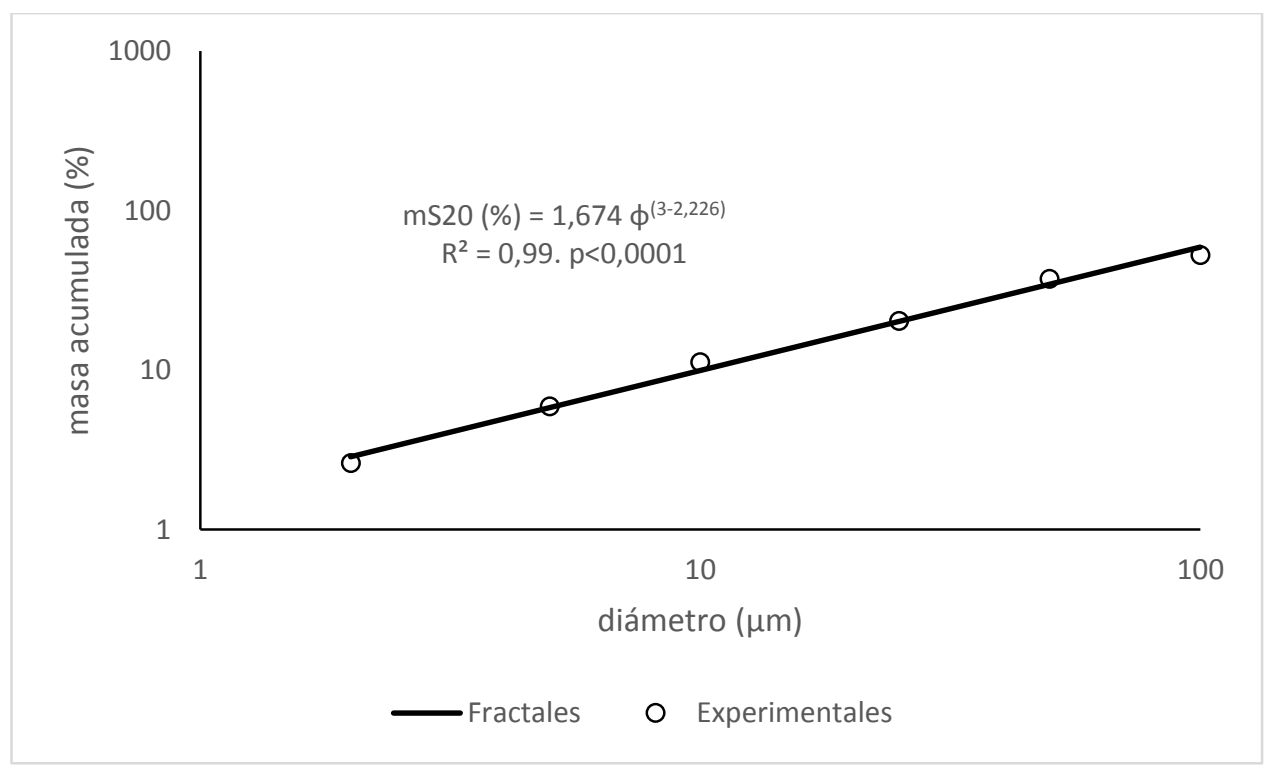
propuesto.

Figura 5.8. Distribución de tamaño de microagregados en función del diámetro $\varnothing(\mu m)$. Modelo fractal 
Los ajustes individuales CMO mejoran respectos al análisis grupal, obteniendo elevados coeficientes de determinación $R^{2}$. Los valores individuales de $C$ y $D$ por suelo se muestran en VinculosIG3CMO CyD individuales.xIsX

\subsection{Discusión del análisis fractal sobre muestras con materia orgánica}

La dimensión fractal parece estar relacionada con el contenido en materia orgánica y en consecuencia puede ser una herramienta que ayude al estudio de los mecanismos de agregación Vivas Miranda (1999). Se aplicó el modelo fractal al estudio de la DTM satisfactoriamente.

La distribución de tamaño de microagregados (DTM) fue descripta por los mismos tipos de modelos que los empleados en el estudio de las DTP (SMO), los Grupos 1 y 3 , mediante el modelo fractal y el Grupo 2 por un modelo lineal.

La DTM, como resultado de la microagregación, se la describió mediante el modelo fractal, aplicado hasta $100 \mu \mathrm{m}$. La masa descripta fue menor que con el tratamiento SMO, como consecuencia del aumento de la proporción de arenas aparentes, no permitiendo la técnica cuantificar tamaños mayores. En trabajos previos Gelati et al. (2010), intentaron cuantificar las arenas aparentes por tamizado, luego de la sedimentación, con inconsistencias, puesto que por dispersión mecánica se obtuvieron valores de arena aparente similares a los de las reales. Por este motivo, se decidió cuantificarlas por diferencia.

Gunal et al. (2011), calculó las dimensiones fractales con diferentes pretratamientos, obteniendo un valor medio de $1,1 \times 10^{-2}$, para la diferencia entre las dimensiones fractales sin materia orgánica ( $\left.D_{s m o}\right)$ y con materia orgánica $\left(D_{\mathrm{cmo}}\right)$. En suelos Pampeanos se obtuvieron, para los mismos pretratamientos, en el Grupo 1 diferencias de 5,6 $10^{-1}$ y para el Grupo 3 de 4,2 $10^{-1}$. Estos resultados fueron de un orden de magnitud mayor respecto a las obtenidas por estos autores. En los suelos estudiados el proceso de microestructuración es apreciable en el orden de la décima de las diferencias de las $D$.

En un análisis análogo a las $D$, pero con el parámetro $C$ al hacer las diferencias entre

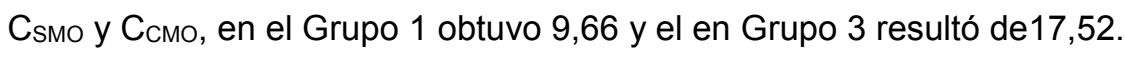

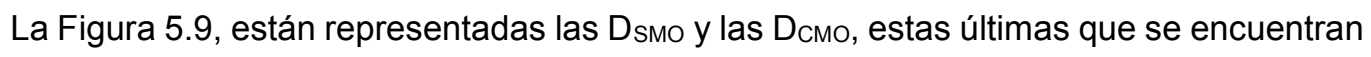
aglutinadas en valores próximos a 2,0 interceptando diferentes curvas teóricas de $\emptyset_{T}$, producto del engrosamiento por efectos de la materia orgánica y la microestructuración resultante.

Los parámetros obtenidos con el modelo fractal fueron sensibles al proceso de microestructuración modificando sus valores significativamente con el pretratamiento. 


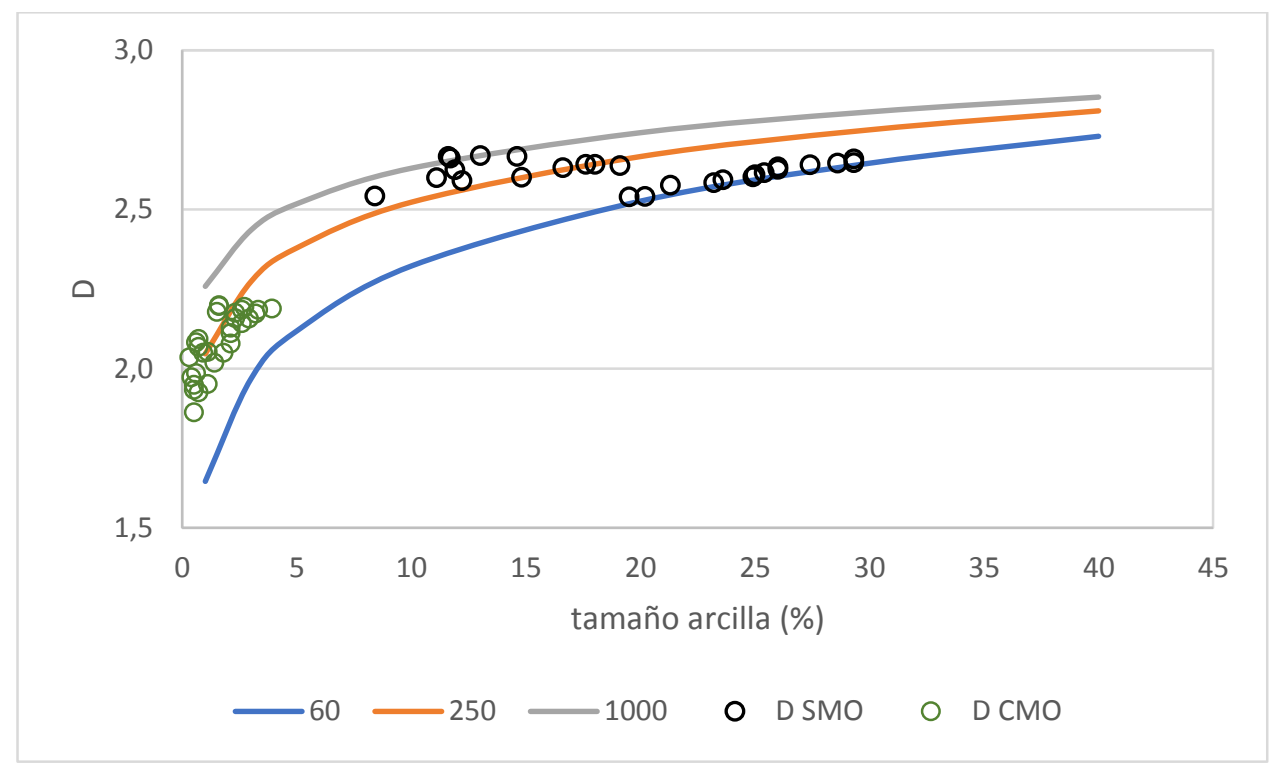

Figura 5.9. Valores de D experimental en función del contenido de arcilla. Se representan las curvas teóricas correspondientes a $D$ para $\emptyset_{T}(\mu m)=60 ; 250$ y 1000 .

\subsection{Comparación grupal SMO - CMO}

Los modelos fractales obtenidos para cada grupo SMO y CMO pueden representarse en un mismo gráfico y comparar las áreas bajo las curvas (Figura 5.10). Integrando las funciones potenciales entre los límites de diámetros máximos evaluados pueden calcularse las áreas. El cociente entre la diferencia de áreas de los tratamientos (SMO - CMO) y el área SMO define el índice de microestructuración (IM) y puede ser expresado en porcentaje.

$$
I M(\%)=\frac{A_{S M O}-A_{C M O}}{A_{S M O}} \times 100 \quad \text { Ecuación } 5-1
$$

Como ejemplo de este índice se presentan en la Figura 5.10 las funciones obtenidas para cada Grupo. Las estimaciones permiten caracterizar al G1FA con un IM $=45,1 \%$ el cual difiere $(p=0,001)$ del $G 1 F$ con $I M=59,4 \%$. El G3 obtuvo un valor medio de $I M=61,4 \%$. 


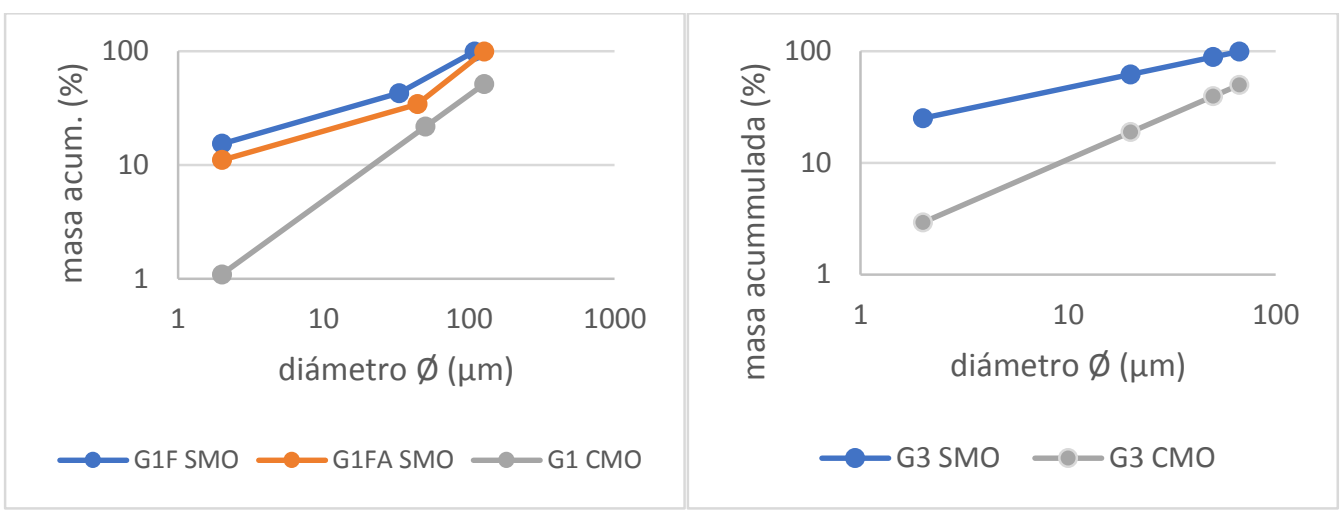

Figura 5.10 Relación entre la masa acumulada y el diámetro de partículas (SMO) - microagregados (CMO) G1FA corresponde al grupo 1 franco arenoso analizando las partículas, G1F corresponde al grupo 1 franco arenoso SMO, G1CMO corresponde a la curva de microagregados. G3 corresponde al grupo 3 analizando las partículas, G3CMO corresponde a la curva de microagregados.

Si se hacemos un análisis entre la condición agrícola y bajo alambrado, G1FA ( $p=0,60)$ y G1F $(p=0,40)$ no presentaron diferencias. El G3 mostró diferencias $(p=0,001)$ entre la condición agrícola (IM=54,2\%) y bajo alambrado (IM 66,7\%). Los valores individuales para cada suelo se encuentran en: Vinculos Indice de microestructuracion por suelo.xlsx

\subsection{Conclusiones del estudio fractal de las curvas de masa acumulada de microagregados}

El proceso de microagregación en los suelos pampeanos hasta un diámetro aparente $\varnothing$ $=100 \mu \mathrm{m}$, puede interpretarse mediante el modelo fractal, la calidad de ajuste decrece igual que la cantidad de masa descripta. El G1CMO, obtuvo una D media de 2,024 en un rango de 1,885 - 2,177. El valor medio de C fue de 0,497 variando entre 0,261 - 1,012. El G3CMO, obtuvo una $D$ media de 2,200 en un rango de 2,032-2,258. El valor medio de $C$ fue de 1,810 variando entre $0,762-2,913$.

En el tamaño de microagregados estudiado, se observó un comportamiento fractal del proceso de microestructuración, los grados de ajuste, así como el alcance de la masa descripta son inferiores a los obtenidos con partículas.

Las curvas de masa acumulada de microagregados en función del diámetro aparente corresponden a clases texturales aparentes más gruesas que las correspondientes a partículas.

Los suelos sedimentan como si fueran de clases texturales más gruesas. 
El efecto de la materia orgánica homogeniza las clases texturales aparentes, volviendo a los suelos franco arenosos en su mayoría. Con estos resultados conceptualmente puede explicarse la cita de Gee y Bauder, (1986) "suelos finos altamente estructurados se comportan como suelos gruesos" el efecto de la materia orgánica, produce microagregados de tamaño arena, con las partículas de arcilla y limo que fueron detectados en las sedimentaciones e imágenes al microscopio".

Los suelos del Grupo 3, con proporciones de arcilla más limo iguales o mayores a al 90 $\%$, la materia orgánica microestructura partículas elementales otorgándole al suelo una proporción de arenas aparentes mayor a la real, lo que favorecería el funcionamiento del suelo, por ejemplo, en el intercambio de fluidos. 
Propuestas

Capítulo 6 


\section{Propuestas}

Se presentan a continuación dos propuestas, derivadas de los resultados de la tesis. La primera, "el empleo del modelo fractal para estimar las DTP de suelos a partir de los contenidos de arena y arcilla" y la segunda, una interpretación desde el modelo fractal para las DTP resultantes al proceso eólico de transporte y depósito del material original en la Pradera Pampeana.

\subsection{Validación del modelo fractal empleando datos de suelos de otras regiones descriptos por INTA}

\subsubsection{Introducción}

La distribución de tamaño de partículas de suelos finos puede ser descripta por el modelo fractal, mientras que suelos gruesos, cuarzosos no ajustarían a este modelo (Bartoli et al. 1991, Barak et al. 1996). En el triángulo textural (Figura 4.1) la función Iso $\varnothing=2000 \mu \mathrm{m}$ lo divide en dos áreas, hacia el origen de coordenadas las combinaciones texturales potencialmente fractales y a la derecha, las combinaciones no fractales. Cuando se obtienen las DTP en laboratorio, del análisis de los resultados se pueden encontrar representantes de ambos sectores, suelos fractales o de fractalidad intermedia, Grupo 3 y 1 respectivamente y suelos no fractales como el Grupo 2. En los casos referidos el ajuste del modelo para cada suelo se obtiene mediante transformaciones y regresiones de los datos experimentales.

Del modelo fractal y la expresión textural se pueden obtener las ecuaciones de la dimensión fractal de fragmentación $D$ (Ecuación 4.9) y del parámetro $C$ (Ecuación 4.11). A partir de la masa de arcillas $m_{\text {arc, }}$ la masa de arenas $m_{A}$, el diámetro aparente de las arcillas y limos, Øarc y ø

Los parámetros del modelo fractal, obtenidos experimentalmente en la tesis no se diferenciaron $C(p=0,99), D(p=0,34)$ de los estimados a partir de estas ecuaciones.

$$
\begin{array}{ll}
D=3-\frac{\log \left(\frac{m_{\text {arc }}}{100-m_{A}}\right)}{\log \left(\frac{\phi_{\text {arc }}}{\phi_{L}}\right)} & \text { Ecuación 4-9 } \\
C=m_{\text {arc }} 2^{D-3} & \text { Ecuación 4-11 }
\end{array}
$$

En la Figura 6.1, para la serie Arrecifes, se presenta la correlación entre los valores de los porcentajes acumulados obtenidos para 2, 5, 10, 25 y $50 \mu \mathrm{m}$ en forma experimental y los mismos estimados con el modelo fractal obtenido con las ecuaciones arriba descriptas. El nivel de correlación fue elevado, $\mathrm{R}=0,999$, la pendiente próxima a uno y la ordenada al origen no diferente de cero. Este nivel de ajuste se repitió para todos los suelos del Grupo 3. 


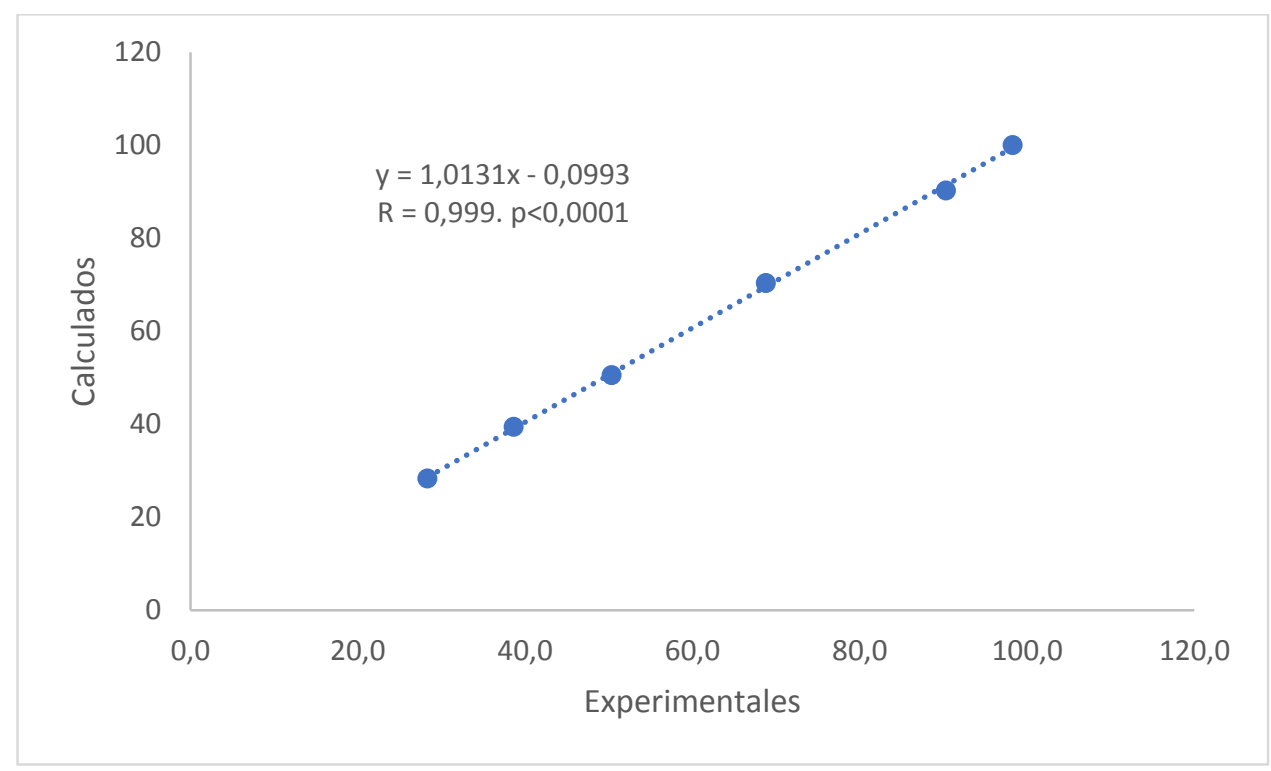

Figura 6.1. Asociación entre los valores experimentales y los valores calculados por el modelo fractal, obtenido a partir de los porcentajes de arena y arcilla, para la serie Arrecifes.

Se planteó como hipótesis que otros órdenes de suelos, además de Molisol tienen distribución de tamaño de partículas fractales.

El objetivo general fue ampliar la modelización fractal a suelos no analizados en la tesis. Para cumplir este objetivo general se plantearon de manera específica:

Obtener los parámetros fractales C y D a partir de los porcentajes de arena y arcilla.

Estimar las DTP y contrastarla con los valores experimentales publicados.

\subsubsection{Materiales y métodos}

De series descriptas por INTA, para suelos de la provincia de Buenos Aires, Santa Fe, Entre Ríos y Formosa se tomaron los valores de arena y arcilla para estimar el modelo fractal descripto en la Introducción. Se eligieron suelos que contaran con la información correspondiente a limos a $20 \mu \mathrm{m}$ y arenas de $75 \mu \mathrm{m}$ para contar por lo menos con cuatro datos experimentales. Con estos datos experimentales se elaboraron las DTP acumuladas y se contrastaron con los valores obtenidos mediante el modelo fractal. Se calculó la correlación para estimar la calidad de ajuste entre los valores experimentales y los estimados por el modelo fractal.

\subsubsection{Resultados y discusión}

Los resultados se presentan en la Tabla 6.1. Se observan elevados coeficientes de correlación en la estimación de la DTP, para los grupos de suelos de las cuatro provincias. 
En algunos suelos se analizaron horizontes superficiales y subsuperficiales. En el caso de los horizontes Bt, se observó una modificación de los parámetros del modelo respecto del horizonte superficial y del material original, la misma fue coherente con el marco teórico. Los cambios en los parámetros fractales indican la modificación de la DTP, consecuencia del proceso de argiluviación. En los Bt, se obtuvieron valores del parámetro "C" mayores a 35, los que no son habituales en horizontes superficiales.

El diámetro teórico máximo $\left(\emptyset_{T}\right)$, en promedio resultó menor a $100 \mu \mathrm{m}$. Cuando se analizaron suelos más gruesos, el modelo fractal resultó efectivo hasta el diámetro aparente de $50 \mu \mathrm{m}$.

Tabla 6.1 Provincia a la que pertenecen las Series analizadas, horizonte estudiado (Hte), A (\%) porcentaje de arena, $L(\%)$ porcentaje de limo y arc (\%) porcentaje de arcilla. C parámetro del modelo fractal estimado, $D$ dimensión fractal de fragmentación estimada, $\emptyset_{T}$, diámetro máximo teórico esperable y $R$ coeficiente de correlación

\begin{tabular}{|c|c|c|c|c|c|c|c|c|}
\hline Pcia/Serie & Hte: & A (\%) & L (\%) & $\operatorname{arc}(\%)$ & $\mathrm{C}$ & D & $\varnothing_{T}(\mu \mathrm{m})$ & $\mathrm{R}$ \\
\hline \multicolumn{9}{|l|}{ Bs As } \\
\hline Río Tala & A1 & 17,1 & 56,8 & 26,1 & 20,350 & 2,641 & 84,3 & 1,00 \\
\hline Rojas & $A$ & 29,5 & 46,8 & 23,7 & 18,741 & 2,661 & 140,4 & 1,00 \\
\hline Rojas & Ap & 27,7 & 49,4 & 22,9 & 17,878 & 2,643 & 124,0 & 1,00 \\
\hline Lima & $A$ & 12,4 & 56,5 & 31,1 & 24,884 & 2,678 & 75,5 & 1,00 \\
\hline Carabelas & Ap1 & 14,7 & 58,5 & 26,8 & 20,886 & 2,640 & 77,8 & 0,99 \\
\hline Carabelas & Bt1 & 12,5 & 49,6 & 37,9 & 31,651 & 2,740 & 83,6 & 0,99 \\
\hline Atucha & Ap & 15,8 & 63,5 & 20,7 & 15,302 & 2,564 & 74,2 & 1,00 \\
\hline Atucha & $A$ & 15,6 & 58,4 & 26 & 20,177 & 2,634 & 79,5 & 1,00 \\
\hline Atucha & Bt1 & 12,4 & 42,3 & 45,3 & 39,303 & 2,795 & 95,4 & 0,99 \\
\hline Pergamino & Ap1 & 12,5 & 64,8 & 22,7 & 16,976 & 2,581 & 68,8 & 1,00 \\
\hline Pergamino & $A$ & 12,1 & 65,1 & 22,8 & 17,050 & 2,581 & 68,0 & 1,00 \\
\hline Solís & Ap1 & 19,7 & 53,6 & 26,7 & 21,064 & 2,658 & 95,0 & 0,99 \\
\hline Solís & A & 15,7 & 56 & 28,3 & 22,372 & 2,661 & 82,7 & 1,00 \\
\hline \multicolumn{9}{|l|}{ Santa Fe } \\
\hline La Rubia & Ap & 2,7 & 69,5 & 27,8 & 21,227 & 2,611 & 53,6 & 1,00 \\
\hline La Rubia & $E$ & 2,5 & 70,4 & 27,1 & 20,570 & 2,602 & 53,3 & 1,00 \\
\hline La Rubia & $C$ & 8,8 & 60,4 & 30,8 & 24,380 & 2,663 & 65,7 & 1,00 \\
\hline Loma Alta & $A$ & 3,5 & 64,5 & 32,0 & 25,230 & 2,657 & 55,5 & 1,00 \\
\hline Pcia/Serie & Hte: & $\mathrm{A}(\%)$ & L (\%) & $\operatorname{arc}(\%)$ & C & D & $\varnothing \mathrm{T}(\mu \mathrm{m})$ & $\mathrm{R}$ \\
\hline Loma Alta & Bt1 & 2,8 & 54 & 43,2 & 36,278 & 2,748 & 56,0 & 1,00 \\
\hline Loma Alta & $C$ & 5,5 & 64,6 & 29,9 & 23,337 & 2,643 & 58,6 & 1,00 \\
\hline Loma Alta & $\mathrm{Ck}$ & 6,8 & 67,6 & 25,6 & 19,382 & 2,599 & 59,6 & 1,00 \\
\hline Humbolt & $\mathrm{A} 1$ & 4 & 66,6 & 29,4 & 22,787 & 2,632 & 55,9 & 1,00 \\
\hline Humbolt & Bt1 & 2,1 & 40 & 57,9 & 51,708 & 2,837 & 56,9 & 1,00 \\
\hline Avellaneda & $A$ & 19,4 & 58 & 22,6 & 17,187 & 2,605 & 86,3 & 0,99 \\
\hline Armstrong & Ap & 4,2 & 75,7 & 20,1 & 14,360 & 2,515 & 54,6 & 1,00 \\
\hline Armstrong & Bt1 & 7,5 & 55,8 & 36,7 & 30,076 & 2,713 & 65,6 & 0,99 \\
\hline Armstrong & $C$ & 5,8 & 80 & 14,2 & 9,448 & 2,412 & 55,3 & 1,00 \\
\hline
\end{tabular}


Tabla 6.2 (Continuación) Provincia a la que pertenecen las Series analizadas, horizonte estudiado (Hte), A (\%) porcentaje de arena, $L(\%)$ porcentaje de limo y arc (\%) porcentaje de arcilla. C parámetro del modelo fractal estimado, $D$ dimensión fractal de fragmentación estimada, $\varnothing T$, diámetro máximo teórico esperable y $R$ coeficiente de correlación

\begin{tabular}{lcccccccc} 
Pcia/Serie & Hte: & $\mathrm{A}(\%)$ & $\mathrm{L}(\%)$ & $\operatorname{arc}(\%)$ & $\mathrm{C}$ & $\mathrm{D}$ & $\emptyset_{\mathrm{T}}(\mu \mathrm{m})$ & $\mathrm{R}$ \\
\hline Hersilia & $\mathrm{A}$ & 2 & 61 & 37 & 29,999 & 2,697 & 53,5 & 1,00 \\
Hersilia & $\mathrm{Bt} 1$ & 1,1 & 44,4 & 54,5 & 47,937 & 2,815 & 53,1 & 1,00 \\
Esperanza & $\mathrm{A}$ & 4,6 & 66,7 & 28,7 & 22,159 & 2,627 & 56,7 & 1,00 \\
Esperanza & $\mathrm{Bt} 1$ & 2 & 38,6 & 59,4 & 53,329 & 2,844 & 56,9 & 1,00 \\
Arocena & $\mathrm{A}$ & 6,8 & 66,1 & 27,1 & 20,771 & 2,616 & 60,1 & 1,00 \\
Arocena & $\mathrm{Bt1}$ & 3,9 & 41,6 & 54,5 & 48,234 & 2,824 & 62,7 & 0,99 \\
Arocena & $\mathrm{C}$ & 9,8 & 69,2 & 21 & 15,343 & 2,547 & 62,8 & 1,00 \\
La Elena & $\mathrm{E}$ & 27,5 & 44 & 28,5 & 23,309 & 2,710 & 151,5 & 1,00 \\
F Tiburcio & $\mathrm{Ap}$ & 31,8 & 48,4 & 19,8 & 15,171 & 2,616 & 135,4 & 1,00 \\
F Tiburcio & $\mathrm{Bw}$ & 38,3 & 39,6 & 22,1 & 17,716 & 2,681 & 227,2 & 0,98 \\
La Elena & $\mathrm{E}$ & 27,5 & 44 & 28,5 & 23,309 & 2,710 & 151,5 & 1,00 \\
La Elena & $\mathrm{Bt} 1$ & 25 & 33 & 42 & 37,070 & 2,820 & 246,9 & 1,00 \\
\hline Entre Ríos & & & & & & & & \\
Ma Dolores & $\mathrm{AB}$ & 2,18 & 50,6 & 47,22 & 40,366 & 2,774 & 55,1 & 0,99 \\
Ramblones & $\mathrm{Ap}$ & 7,5 & 61,3 & 31,2 & 24,690 & 2,662 & 63,0 & 0,98 \\
Ma. Grande & $\mathrm{Ap}$ & 1,54 & 66,88 & 31,58 & 24,721 & 2,647 & 52,2 & 0,99 \\
San Pablito & $\mathrm{Bt} 1$ & 2,71 & 46,78 & 50,51 & 43,860 & 2,796 & 57,2 & 0,98 \\
El Recreo & $\mathrm{Ap}$ & 1,71 & 57,89 & 40,4 & 33,361 & 2,724 & 53,2 & 1,00 \\
Est El Sauce & $\mathrm{A} 1$ & 6,8 & 54,9 & 38,3 & 31,625 & 2,724 & 64,5 & 1,00 \\
Damasio & $\mathrm{Ap}$ & 3,6 & 63,4 & 33 & 26,198 & 2,667 & 55,8 & 0,99 \\
Viale & $\mathrm{A} 1$ & 1,54 & 60,54 & 37,92 & 30,877 & 2,704 & 52,7 & 1,00 \\
Arrua & $\mathrm{A}$ & 2,7 & 71,2 & 26,1 & 19,660 & 2,591 & 53,5 & 0,98 \\
Alcaraz & $\mathrm{Ap}$ & 1,6 & 72,9 & 25,5 & 19,066 & 2,580 & 52,0 & 0,98 \\
Viraró & $\mathrm{A}$ & 0,7 & 69,6 & 29,7 & 22,902 & 2,625 & 50,9 & 1,00 \\
\hline Formosa & & & & & & & & \\
Perín & $\mathrm{A}$ & 6,6 & 71,2 & 22,2 & 16,292 & 2,554 & 58,3 & 1,00 \\
Palo Santo & $\mathrm{E} 1$ & 31 & 49,1 & 19,9 & 15,225 & 2,614 & 130,7 & 1,00 \\
Zapala & $\mathrm{A}$ & 26,9 & 54,2 & 18,9 & 14,124 & 2,580 & 105,4 & 0,99 \\
Zapala & $\mathrm{E}$ & 25,7 & 54,7 & 19,6 & 14,711 & 2,586 & 102,5 & 1,00 \\
Magaik & $\mathrm{E}$ & 12,9 & 46,5 & 40,6 & 34,446 & 2,763 & 89,5 & 0,98 \\
Coatín & $\mathrm{A}$ & 18,4 & 57,7 & 23,9 & 18,347 & 2,619 & 85,2 & 0,99 \\
Bañado & $\mathrm{E}$ & 17,6 & 46 & 36,4 & 30,528 & 2,746 & 107,2 & 0,99
\end{tabular}

El modelo fractal teórico, calculado a partir de los porcentajes de arena y arcilla, permitió estimar con elevado nivel de correlación la distribución de tamaño de partículas de 56 horizontes de suelos de diferentes ámbitos del país, abarcando cuatro Órdenes: Molisoles, Entisoles Alfisoles y Vertisoles.

Para provincia de Buenos Aires y Santa Fe, los datos experimentales propios y de otros autores, permitieron validar el modelo fractal para describir la DTP de estos suelos analizados. 
La metodología propuesta logró profundizar la aplicación del modelo fractal a horizontes E, Bt y $C$, los cuales no son habitualmente descriptos en trabajos sobre fractales. Los parámetros $C$ y $D$ obtenidos fueron coherentes con el marco teórico, notando por ejemplo el incremento de arcillas producto del proceso de argiluviación. En casos de suelos más gruesos, el modelo fractal describió hasta el diámetro aparente de $50 \mu \mathrm{m}$, similar a los casos encontrados en el G1F y G1FA, donde se pudo analizar con más detalle puesto que se contaban con datos experimentales de arenas.

\subsubsection{Conclusión}

La distribución de tamaño de partículas de los suelos estudiados es promisoriamente de tipo fractal. Se sugiere cuantificar experimentalmente la distribución de tamaño de partículas de suelos representativos para confirmar estos resultados.

\subsection{Alcances y propuesta de trabajos a futuros}

Para suelos de otras provincias, con diferentes materiales originales y procesos de formación sería necesario estudiar detalladamente el comportamiento de las distribuciones de tamaño de partículas y verificar si el modelo fractal describe a las mismas.

Se propone un proyecto de trabajo interinstitucional con el relevamiento de las series más representativas en cuanto a superficie o uso, analizarlas experimentalmente en forma detallada y contrastarlas con el modelo propuesto.

Esta herramienta permitiría conocer más y mejor la naturaleza de nuestros suelos, los parámetros $C$ y $D$ podrían ser parte de la descripción de los mismos, obteniéndose la clase textural como también la curva acumulada de partículas. 
6.3 Modelización de la distribución de tamaños de partículas en la Pradera Pampeana

En el norte de la región Pampeana se distinguen, con criterios sedimentológicos y geomorfológicos, básicamente dos regiones: la Pampa Ondulada y la Pampa Arenosa (Cappanini y Dominguez,1961). Más recientemente Iriondo $(1990,1992)$ e Iriondo y Kröhling $(1995,1996)$ distinguen, dentro del que denominan "Sistema eólico pampeano" el 'Mar de arena' hacia el centro-oeste y una faja periférica de "loess" (Subsistema loéssico pampeano) hacia el este. Esta zonación sería consecuencia de un proceso de selección granulométrica como resultado del transporte eólico desde las fuentes de aporte, con una sedimentación proximal de arena y una distal de limo. Es así que el material parental de los suelos grada desde limos loéssicos en el este hasta arenas limosas en el oeste (González Bonorino, 1966) en Morrás y Cruzate (2000).

Las clases texturales y la afinidad por el modelo fractal de cada Grupo, evidencian las consecuencias de la sedimentación diferencial en los suelos de la tesis

Se acepta que los suelos evolucionaron a partir de un material original, que sería una porción más o menos diferenciada, debido a los procesos de transporte y sedimentación de lo que llamaremos Material Generador. Este Material Generador en principio tendría la colección de partículas completa.

\subsubsection{Modelización fractal a partir de un hipotético Material Generador}

Supongamos un Material Generador de naturaleza fractal qué arriba a la Pradera Pampeana y con una distribución de tamaño de partículas que responde al siguiente modelo:

$$
\begin{aligned}
& M G=12,00 \emptyset^{0,38} \quad \mathrm{D}=2,62 \\
& 2<\emptyset(\mu \mathrm{m})<150
\end{aligned}
$$

En base a la experiencia de los resultados obtenidos en la tesis se adoptaron los valores de C, D y el límite superior de $150 \mu \mathrm{m}$.

Supondremos, la formación de dos suelos modales: uno de la Pampa Arenosa y otro de la Pampa Ondulada.

Para desarrollar el razonamiento consideraremos que el transporte eólico afecta, al Material Generador con una tasa transporte y depósito del $80 \%$ a las partículas menores a 75 $\mu \mathrm{m}$ y $0 \%$ a las comprendidas entre 75 y $150 \mu \mathrm{m}$. Esto significa que el material original de los Suelos de la Pampa Arenosa (SPA) se forma con el $20 \%$ remanente de las partículas hasta 75 $\mu \mathrm{m}$ y el $100 \%$ de las partículas entre 75 y $150 \mu \mathrm{m}$ del Material Generador. 
Por otra parte, el material original sobre el que habrían evolucionado los suelos de la Pampa Ondulada (SPO) estarían formados por el $80 \%$ de las partículas menores a $75 \mu \mathrm{m}$ del Material Generador que siguieron en suspensión hasta depositarse. Las DTP que resultarían del Material Generador y de los suelos de la Pampa Arenosa y Ondulada calculadas pueden consultarse en la Tabla 6.2 y observarse en la Figura 6.2.

El proceso de transporte eólico sobre el Material Generador produjo dos materiales originales, de naturaleza común pero diferente distribución de tamaños de partículas. Los SPA, presentan un déficit en las partículas finas y un enriquecimiento residual de la fracción gruesa (respecto al Material Generador). La distribución de tamaño de partículas es de tipo sigmoidea y un modelo fractal la describe parcialmente (Figura 6.2). En el ejemplo, el modelo quedó definido con los siguientes parámetros y acotado al dominio a los diámetros 2 a $75 \mu \mathrm{m}$, alcanzando a describir el $39,9 \%$ de la masa total.

$$
\begin{aligned}
& S P A=7,73 \emptyset^{0,38} \quad D=2,62 \\
& 2<\emptyset(\mu \mathrm{m})<75
\end{aligned}
$$

Si se extrapola el modelo a diámetros mayores ocurre una subvaloración de la fracción más gruesa (igual a lo observado en los datos experimentales de la tesis). La dimensión fractal de fragmentación estimada resulta igual a la del Material Generador.

Por otra parte, los suelos de la Pampa Ondulada, fueron descriptos por el siguiente modelo:

$$
\begin{gathered}
S P O=19,39 \emptyset^{0,38} \quad D=2,62 \\
2<\emptyset(\mu \mathrm{m})<75
\end{gathered}
$$

El modelo alcanza a describir el $100 \%$ de la masa y nuevamente estima la dimensión fractal de fragmentación " $D$ " igual a la del Material Generador. 
Tabla 6.3. Distribución de tamaño de partículas, con una tasa de transporte eólico del 80\% para el Material Generador (MG), suelo de la Pampa Arenosa (SPA), modelización fractal del suelo de la Pampa Arenosa (SPA(F)) y del suelo de la Pampa Ondulada (SPO)

\begin{tabular}{ccccc}
$\varnothing(\mu \mathrm{m})$ & MG (\%) & SPA (\%) & SPA(F) (\%) & SPO (\%) \\
\hline 2 & 15,6 & 10,1 & 10,1 & 25,2 \\
20 & 37,5 & 24,1 & 24,1 & 60,5 \\
50 & 53,1 & 34,2 & 34,2 & 85,7 \\
75 & 61,9 & 39,9 & 39,9 & 100,0 \\
100 & 69,1 & 62,9 & & \\
150 & 80,6 & 100,0 & &
\end{tabular}

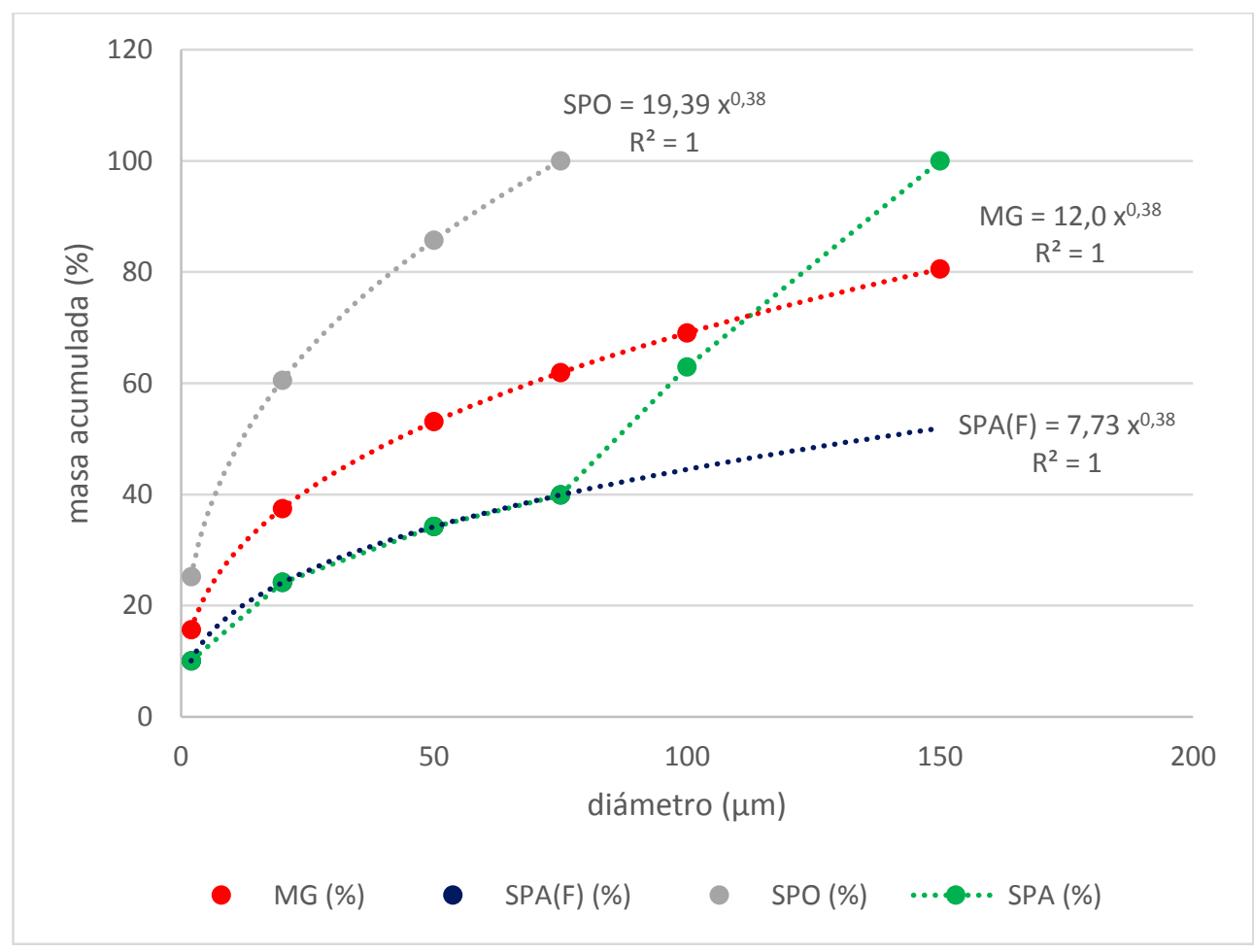

Figura 6.2. MG Material Generador de naturaleza fractal. SPA suelo de la Pampa Arenosa, enriquecido residualmente por fracciones gruesas. SPA(F) modelo fractal parcial hasta el 39,9\% de la masa, entre 2 y $75 \mu \mathrm{m}$ para SPA. SPO suelo de la Pampa Ondulada, de naturaleza fractal entre 2 y $75 \mu \mathrm{m}$.

Los resultados obtenidos con el modelo propuesto son coherentes con el marco teórico, Ios SPA, presentan un valor de C menor que el Material Generador como consecuencia del enriquecimiento residual de partículas gruesas. Mientras que, en los SPO, el valor de $\mathrm{C}$ es mayor puesto que fue enriquecido en partículas finas por el proceso de selección y transporte. Las dimensiones fractales de fragmentación son iguales, puesto que pertenecen a porciones del mismo Material Generador. El comportamiento matemático del modelo no se altera con el valor de la tasa transporte y esto mantiene las $D$ inalteradas. 
Los SPA pueden asociarse a los del G1FA y los SPO con los del G3 descriptos en la tesis.

\subsubsection{Tasas variables de transporte}

Si al modelo anterior, le asignamos tasas de transporte diferenciales según rangos de tamaños de partículas como, por ejemplo:

\begin{tabular}{cc}
$\varnothing(\mu \mathrm{m})$ & Tasa (\%) \\
\hline $2-30$ & $85 \%$ \\
$30-50$ & $70 \%$ \\
$50-100$ & $40 \%$
\end{tabular}

aplicadas al Material Generador, se obtienen nuevas distribuciones de tamaños de partículas para los SPA y SPO, que pueden encontrarse en la Tabla 6.3 y observarse en la Figura 6.3 .

El modelo fractal para los suelos de la Pampa Arenosa, quedaría definido de la siguiente manera:

$$
\begin{gathered}
S P A(F)=5,09 \emptyset^{0,416} \quad D=2,584 \\
\mathrm{R}^{2}=0,99 . \\
2<\varnothing(\mu \mathrm{m})<50
\end{gathered}
$$

El mismo describe hasta los $50 \mu \mathrm{m}$ alcanzando el $28,1 \%$ de la masa acumulada. De proyectarlo a diámetros mayores reitera una subvaloración sistemática de las fracciones más gruesas. La D no es exactamente igual a la del Material Generador y aparecen ligeros desvíos de los datos en torno al modelo.

Los suelos de la Pampa Ondulada responden al siguiente modelo:

$$
\begin{gathered}
S P O=22,44 \emptyset^{0,359} \quad D=2,641 \\
\mathrm{R}^{2}=0,995 . \\
2<\emptyset(\mu \mathrm{m})<75
\end{gathered}
$$

En este grupo, el modelo presenta una D que también difiere a la del Material Generador, y se observan ligeros desvíos alrededor de los valores propuestos por el modelo similar en SPA. 
En los trabajos de Tyler y Wheatcraft (1992), Bitelli et al. (1999), Filgueira et al. (2006) y Behzad (2015) se observan estos desvíos entre los datos experimentales y el modelo.

Tabla 6.4. Distribución de tamaño de partículas acumulado expresado en porcentaje, con tasas variables de transporte eólico. Ø: diámetro de partículas en $\mu m$. MG, Material Generador; SPA suelo de la Pampa Arenosa; SPA(F) suelo de la Pampa arenosa estimada con el modelo fractal; SPO suelo de la Pampa Ondulada.

\begin{tabular}{ccccc}
$\varnothing(\mu \mathrm{m})$ & $\mathrm{MG}(\%)$ & $\mathrm{SPA}(\%)$ & $\mathrm{SPA}(\mathrm{F})(\%)$ & $\mathrm{SPO}(\%)$ \\
\hline 2 & 15,6 & 7,0 & 7,0 & 28,1 \\
5 & 22,1 & 10,0 & 10,0 & 39,8 \\
10 & 28,8 & 13,0 & 13,0 & 51,8 \\
20 & 37,5 & 16,9 & 16,9 & 67,4 \\
30 & 43,7 & 19,7 & 19,7 & 78,6 \\
40 & 48,7 & 24,2 & 24,2 & 86,1 \\
50 & 53,1 & 28,1 & 28,1 & 92,5 \\
60 & 56,9 & 35,0 & & 95,7 \\
75 & 61,9 & 44,0 & & 100,0 \\
100 & 69,1 & 65,5 & & \\
150 & 80,6 & 100,0 & &
\end{tabular}

Con estos valores hipotéticos de tasas variables de transporte y depósito, se obtiene, de manera teórica a través del modelo, aproximaciones a las DTP que se asemejan a los datos experimentales observados en la tesis.

Puede plantearse, que de un Material Generador de naturaleza fractal se forman materiales originales diferentes por fraccionamiento, que tendrán " $D$ " similares, diferente rango de tamaños de partículas y en consecuencia, distintas " $C$ ".

Cuando las tasas de transporte y depósito del Material Generador son variables, el modelo teórico predice alteraciones en el comportamiento potencial de las DTP, análogo a lo observados en la bibliografía y en los datos experimentales de la tesis. 


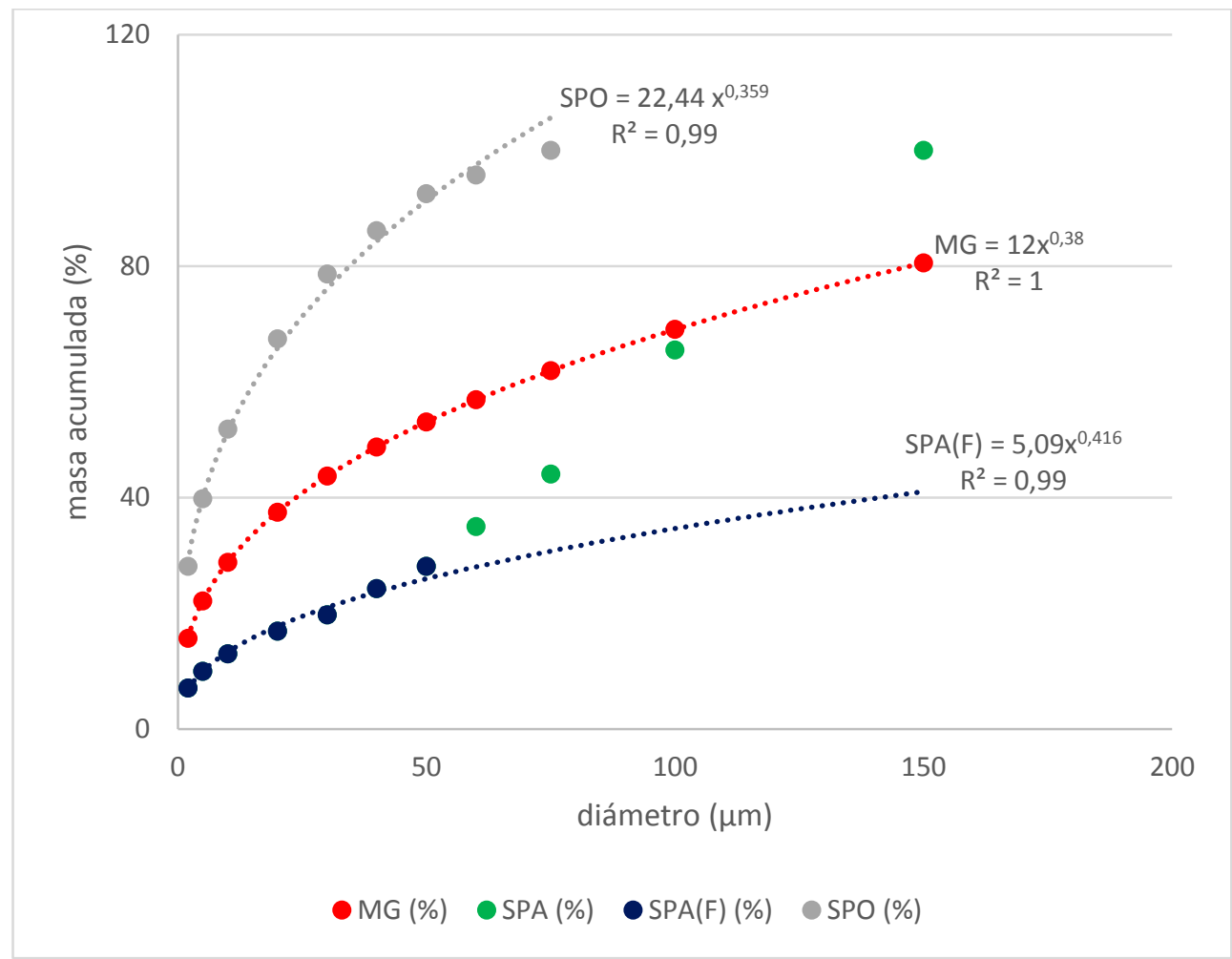

Figura 6.3. DTP teórica de suelos resultantes de considerar tasas variables de transporte eólico.

\subsubsection{Conclusión}

La modelización realizada demuestra que a partir de un Material Generador de naturaleza fractal, por transporte y sedimentación diferencial, en este caso eólica, es esperable encontrar:

-Materiales originales de naturaleza fractal, los más seleccionados y distantes al origen

-Materiales originales de naturaleza fractal intermedia, solo una parte del material original puede ser descripto por un solo modelo fractal

-Materiales originales de naturaleza no fractal, aquellos que se transformaron residualmente en los materiales originales más gruesos por pérdida de las partículas más finas. 
6.4 Modelización de la distribución de tamaños de partículas en la Pradera Pampeana, con los datos experimentales

Es de consenso en la bibliografía que los suelos finos son descriptos por el modelo fractal mientras que suelos más ricos en arenas, son parcialmente o no son descriptos por este modelo. (Bartoli et al. 1991; Tyler y Wheatcraft, 1992; Bitelli et al. 1999; Millan et al. 2003 y Filgueira et al. 2006).

Los resultados experimentales obtenidos correspondieron a:

Grupo 3, suelos fractales de la Pampa Ondulada de textura franco limosa.

Grupo 1 (G1F y G1FA), suelos de fractalidad intermedia de la Pampa Arenosa. Se necesitó recurrir a dos modelos fractales para suplir los déficits de partículas medias a finas.

Grupo 2, suelos no fractales de la Pampa Arenosa con texturas areno franco, residualmente gruesos.

Los Grupos 1 y 3 mostraron diferencias en el parámetro "C" ( $p<0,0001)$, pero similitud en la dimensión fractal de fragmentación " $D$ " ( $p=0,35)$.

Estos resultados pueden interpretarse, como dimensiones fractales de fragmentación no diferentes se asocian a porciones de un mismo Material Generador. En la modelación teórica fractal las $D$ de los grupos son similares a las del Material Generador, mientras que valores de " $\mathrm{C}$ " diferentes, indican distintas granulometrías, consecuencia de la sedimentación diferencial del transporte eólico. Esto estaría explicado en el modelo fractal teórico antes descripto (ítem 6.2. Modelización de la distribución de tamaños de partículas en la Pradera Pampeana. Teórica).

Para estimar la distribución de tamaño de partículas del Material Generador, se consideró que los suelos evolucionaron sobre Loess del mismo ciclo, que fue el único material original y que no hubo procesos de neoformación de arcillas.

Con los datos experimentales se propone el ejercicio inverso a la modelización teórica anterior, puesto que ahora tenemos las DTP resultantes de la sedimentación diferencial y debemos reconstruir el Material Generador (Figura 6.4). Para esto, se sumaron las proporciones granulométricas de los grupos hasta $200 \mu \mathrm{m}$ (fractales y no fractales) y se estimaron los porcentajes de la distribución de tamaños de partículas del Material Generador resultante (Tabla 6.4). 
Tabla 6.5 Distribución de tamaño de partícula en porcentaje acumulado del MG Material Generador, G1F Grupo 1 de suelos franco, G1FA Grupo 1 de suelos franco arenosos, G2 Grupo 2 y G3 Grupo 3.

\begin{tabular}{crrrrr}
$\varnothing(\mu \mathrm{m})$ & MG (\%) & G1F (\%) & G1FA (\%) & G2 (\%) & G3 (\%) \\
\hline 2 & $\mathbf{1 5 , 3}$ & 15,5 & 11,0 & 7,1 & 25,2 \\
5 & $\mathbf{2 1 , 1}$ & 21,8 & 15,5 & 7,9 & 36,1 \\
10 & $\mathbf{2 7 , 2}$ & 28,3 & 19,9 & 9,1 & 47,3 \\
20 & $\mathbf{3 5 , 4}$ & 36,8 & 25,7 & 11,6 & 62,1 \\
33 & $\mathbf{4 3 , 0}$ & 44,4 & 30,9 & 14,7 & 75,5 \\
44 & $\mathbf{4 8 , 9}$ & 52,0 & 34,3 & 17,4 & 84,4 \\
66 & $\mathbf{6 2 , 2}$ & 65,5 & 51,8 & 22,9 & 99,2 \\
105 & $\mathbf{7 7 , 8}$ & 84,8 & 82,5 & 32,5 & 99,5 \\
122 & $\mathbf{8 5 , 9}$ & 92,3 & 96,1 & 42,4 & 99,6 \\
130 & $\mathbf{8 7 , 9}$ & 95,5 & 96,3 & 46,9 & 99,6 \\
200 & $\mathbf{9 9 , 2}$ & 96,6 & 97,2 & 88,1 & 99,9
\end{tabular}

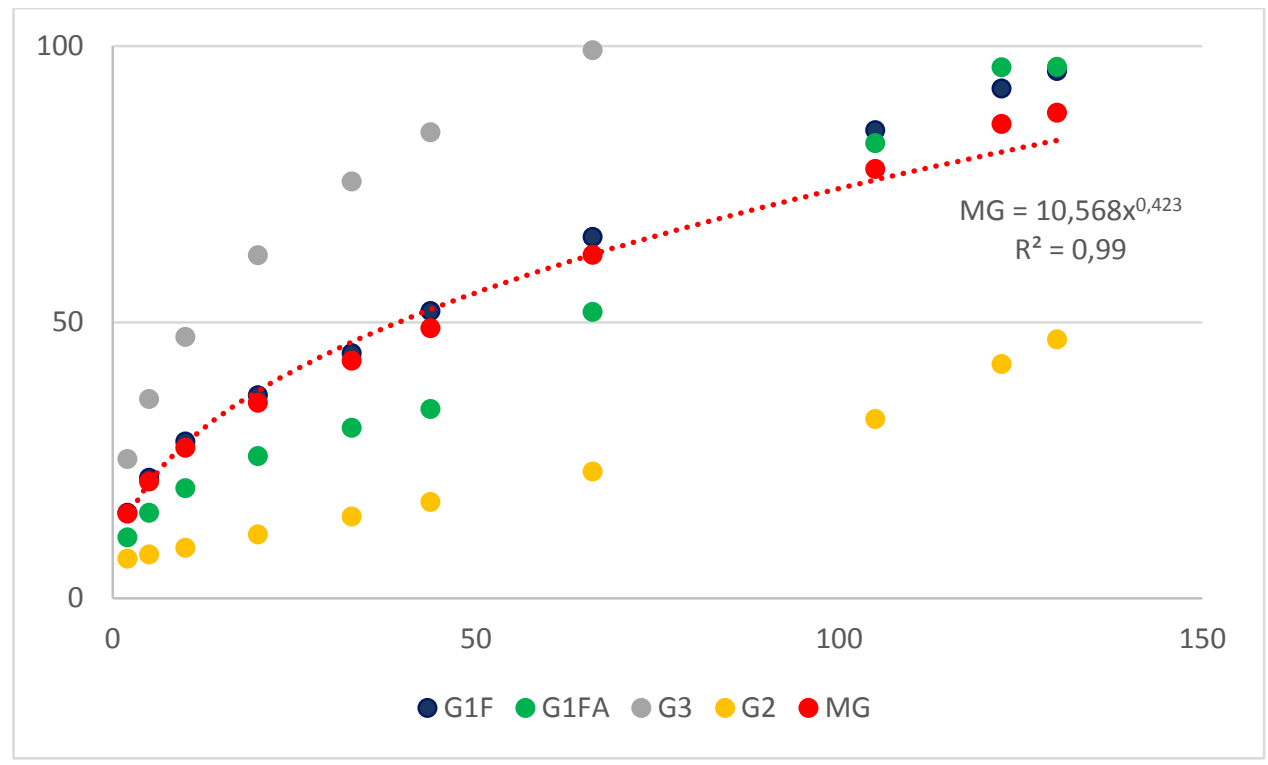

Figura 6.4. Distribución de tamaño de partículas del Material Generador resultante de integrar las partículas de los Grupos de suelos G1F, G1FA, G2 y G3. 
El Material Generador estimado con los datos experimentales correspondientes a los Grupos (Figura 6.4), presenta una distribución de tamaño de partículas de tipo fractal $(p<0,0001)$, elevado ajuste del coeficiente de determinación $R^{2}=0,99$ y alcanza a describir hasta parte del dominio de las arenas finas.

Los parámetros del modelo propuestos resultaron:

$$
\begin{aligned}
& \text { Material Generador }(\%)=10,568 \emptyset^{0,423} \quad D=2,577 \\
& 2<\varnothing(\mu \mathrm{m}) \leq 110 \\
& \mathrm{R}^{2}=0,99 . \mathrm{p}<0,0001
\end{aligned}
$$

Estos resultados son consistentes con el marco teórico: Cuando "C" aumenta, se incrementa el contenido de arcillas de los suelos y disminuye el diámetro máximo teórico $\left(\varnothing_{\mathrm{T}}\right)$ hasta donde la distribución se comporta como fractal. El Material Generador tiene similar D al de los Grupos. Por otra parte, el valor de C, es menor respecto al Grupo 3 y mayor que al del Grupo 1 (G1F-G1FA).

Podría interpretarse que el Material Generador obtenido con los datos experimentales para los suelos de la Pradera Pampeana, tenía una naturaleza fractal, el transporte eólico con la sedimentación diferencial generó: suelos no fractales (Grupo 2), suelos con fractalidad intermedia (Grupo 1) y suelos fractales (Grupo 3), en diferentes zonas de la Pradera Pampeana.

A medida que el material se va haciendo más fino, la DTP de los suelos es mejor descripta por el modelo potencial.

El modelo fractal permitió, interpretar el origen común de los materiales originales de los suelos templados Pampeanos, con misma " $\mathrm{D}$ " y las diferencias en granulometrías mediante el parámetro "C".

\subsection{Conclusión}

Los datos experimentales obtenidos en este trabajo para las distribuciones de tamaño de partículas, agrupados en $\mathrm{G} 1, \mathrm{G} 2$ y G3, han podido ser explicados a partir del transporte eólico, que distribuyó y depositó el material original de los suelos Pampeanos. El Material Generador, obtenido a partir de la colección de las partículas de los Grupos analizados y la sedimentación diferencial, permitió explicar las distribuciones de tamaño de partículas encontradas en función de las tasas de transporte y depósito, asociándose también con las ligeras alteraciones de los datos experimentales al modelo propuesto. 
De un Material Generador de naturaleza fractal es esperable encontrar suelos fractales, suelos de fractalidad intermedia, aquellos necesitan más de un modelo fractal para describirlos, y suelos no fractales

\subsubsection{Conclusión de los datos experimentales}

La distribución de tamaño de partículas de los suelos de la Pradera Pampeana, puede ser explicada mediante el modelo fractal.

El modelado teórico expuesto anteriormente es corroborado por los datos experimentales. El Material Generador obtenido de la suma de partículas es de naturaleza fractal hasta los $110 \mu \mathrm{m}$.

Los suelos del Grupo 3, representan a los de naturaleza fractal completa, formados con tamaños de partículas menores y están más distantes del origen.

Los suelos del Grupo 1, son aquellos que tienen naturaleza fractal intermedia, perdieron partículas en los tamaños menores que son las que formaron a los suelos del Grupo 3 y necesitan de más de un modelo para explicar la distribución de tamaño de partículas hasta el $95 \%$ de la masa acumulada.

Los suelos del Grupo 2, son aquellos que perdieron la mayor parte de partículas más finas, se transformaron en suelos residualmente más gruesos y la distribución de tamaño de partículas acumulada no responden al modelo fractal.

Los resultados experimentales son coherentes con el marco teórico y el modelo anterior propuesto.

El análisis grupal permitió obtener información que resulta de mayor valor que los datos del valor de la $\mathrm{C}$ y $\mathrm{D}$ individuales. 
Recapitulación

Capítulo 7 


\section{Recapitulación}

\subsection{Hipótesis}

1. La distribución de partículas y microagregados de suelos, de clima templado de la pradera pampeana, independientemente de la clase textural y el contenido de materia orgánica, responde a una distribución tipo fractal.

Conclusión: El Material Generador de los suelos pampeanos fue de naturaleza fractal hasta las arenas finas $(110 \mu \mathrm{m})$. El análisis del proceso de distribución de este Material Generador por acción eólica, predice la formación de suelos fractales, como los del Grupo 3, suelos de fractalidad intermedia, como el Grupo 1, que necesitaron de dos modelos para describir el 95\%, puesto que los faltantes de partículas se depositaron más lejos formando los suelos del Grupo 3, y suelos no fractales, como en el Grupo 2 que son residualmente más gruesos.

La distribución de tamaños de microagregados fue fractal hasta los $100 \mu \mathrm{m}$. Los suelos microagregados mostraron uniformidad en los Grupos en cuanto a las clases texturales aparentes. El proceso de microagregación, redujo la masa descripta por el modelo fractal, como consecuencia del pasaje a clases texturales aparentes más gruesas

2. La dimensión fractal de fragmentación $D$, es sensible al tamaño y cantidad de microagregados - partículas y, por ende, al contenido de materia orgánica del suelo.

Conclusión: El proceso de microestructuración modificó la distribución de tamaño de microagregados respecto a la de partículas y estas diferencias fueron evidenciadas en los parámetros del modelo fractal para cada condición, por lo tanto, el modelo fractal resultó sensible a estos procesos. En los tres Grupos se observó el incremento de la fracción de arenas aparentes respecto de las reales y la disminución de los limos y arcillas aparentes respecto de las reales. Sin embargo, las fracciones aparentes de arena limo y arcilla se comportaron diferente respecto de la materia orgánica según el Grupo analizado.

En el Grupo 1, francos y franco-arenosos, las relaciones entre arenas y limos aparentes en función de la fracción orgánica no mostraron significancias. El Grupo 2 se comportó de similar manera. Es recomendable ampliar el número de suelos estudiados para definir adecuadamente el funcionamiento de las fracciones aparentes y la fracción orgánica en estos tipos de suelos

El Grupo 3, suelos franco-limosos y franco-arcillo-limosos, mostraron relaciones significativas entre las fracciones aparentes de arena limo y arcilla con el carbono orgánico asociado a la fracción mineral. Esta fracción del carbono fue más sensible que el carbono orgánico total. 
3. Se puede obtener información sobre el estado de la microestructuración de un suelo a través del estudio de la distribución de partículas, microagregados y el análisis fractal de fragmentación.

Conclusión: Puesto que la modificación de las distribuciones de tamaño de partículas y microagregados es tan contrastante que aparecen diferencias de 0,45 unidades en el parámetro del escalamiento " $D$ ", se puede obtener información sobre el proceso de microestructuración.

El modelo mejora en la calidad descriptiva del proceso analizado si se incorpora el parámetro "C". Su valor está definido por el diámetro máximo teórico fractal. Cuanto mayor es el grado de microagregación menor resulta el valor de C. Esto es relativo para cada grupo puesto que depende de cuál es el valor de partida del suelo analizado sin materia orgánica.

Se puede detectar perfectamente la microagregación de partículas finas (arcillas y limos) y el pasaje a microagregados de tamaño arena a través del modelo fractal. La diferencia de áreas entre las curvas SMO - CMO, relacionada con el área SMO, estima en porcentaje la microestructuración producida por la materia orgánica.

\subsection{Objetivos \\ 7.3 Generales}

1. Investigar el comportamiento fractal de la distribución de tamaños de partículas (DTP) y la distribución de tamaño de microagregados (DTM) del suelo.

Se cumplieron satisfactoriamente

2. Investigar el proceso de microagregación en suelos con texturas contrastantes, resultante de la unión de partículas elementales y la formación de microagregados por acción de la materia orgánica.

Se realizó satisfactoriamente. 
3. Asociar la distribución de tamaño de partículas con el material original y los procesos de transporte y sedimentación.

Se obtuvo un modelo que explica las distribuciones de tamaño de partículas de los suelos templados de la pradera Pampeana, empleando el modelo fractal y el transporte-sedimentación diferencial producto de la acción eólica.

\subsection{Particulares \\ 7.5 Objetivos particulares}

1.8.4 Realizar un modelo fractal que interprete y explique el proceso la sedimentación diferencial del material original en la Pradera Pampeana.

1. Obtener por sedimentación la distribución de tamaño de partículas y microagregados de suelos pampeanos e interpretar las curvas de distribución resultantes, mediante la geometría fractal, obteniendo las dimensiones de fragmentación $D$ y el parámetro $C$.

Se cumplió satisfactoriamente, se incorporó el uso de la constante $C$, que nunca fuera usada en trabajos de investigación publicados, logrando diferenciar exitosamente a los suelos de los Grupos.

Se obtuvieron las relaciones entre los parámetros C-arcilla\% y D-arcilla\%, a partir del marco teórico, esclareciendo desde la teoría el tipo de relación matemática existente.

Se enriqueció la investigación con la aplicación del método de análisis y calificación de las muestras empleado en sedimentología con el parámetro Phi y el desvío estándar.

\section{Investigar si el modelo fractal es un indicador sensible para cuantificar el proceso de microagregación producido por la materia orgánica}

Las diferencias encontradas entre las curvas correspondientes a la distribución de tamaño de partículas y microagregados fueron evidentes y se reflejaron en los parámetros $C$ y $D$ en cada caso.

La utilización del método empleado en sedimentología (masa acumulada en función del parámetro Phi) cuenta con la ventaja de estar probado y con escalas calificadoras establecidas 
y consensuadas y en este trabajo resultaron útiles. La modelización fractal aún no cuenta con calificaciones precisas de sus parámetros.

La relación porcentual entre las áreas de las curvas SMO-CMO, resultó un índice en porcentaje que indica la microestructuración

3. Estudiar al microscopio la presencia de partículas y microagregados en las diferentes fracciones granulométricas, principalmente el dominio arcilla y limo, para los diferentes pretratamientos.

El proceso de microestructuración detectado al comparar sedimentaciones con materia y sin materia orgánica y reflejado en los parámetros $C$ y $D$, se observó en las tomas fotográficas realizadas al microscopio. Las muestras con materia orgánica, mostraron heterogeneidad en formas, tamaño y arreglo de partículas que pudieron distinguirse en las observaciones al microscopio, las fotos fueron una parte del estudio.

Se observó la exhaustividad del pretratamiento sin materia orgánica y la complejidad de formas y tamaños producto de la microestructuración. La superposición de partículas y los diámetros máximos.

Se estudió con detalle la formación de microagregados o pseudopartículas a partir de partículas en observación y medida de micrografías mediante microscopía óptica y uso de cámara LCD con conexión a monitor de alta resolución. Algunos de los resultados obtenidos se expusieron en esta tesis.

\section{Realizar un modelo fractal que interprete y explique el proceso la sedimentación diferencial del material original en la Pradera Pampeana.}

Se realizó satisfactoriamente.

\subsection{Aplicaciones}

Se obtuvo un modelo de tipo fractal que permitió explicar en forma teórica las distribuciones de tamaños de partículas de los suelos esperables a partir del transporte y depósito del material original por medio eólico. Los resultados experimentales coinciden con el modelo. 
Se obtuvo un modelo para estimar la DTP de los suelos a partir de los porcentajes de arena y arcilla, el mismo se contrastó con 30 suelos publicados por INTA.

Se aplicó la metodología fractal al estudio de las DTP entre horizontes contrastantes de un suelo, por ejemplo, Ap y Bt.

\subsection{Futuras líneas de investigación derivadas de la tesis}

Investigar la aplicación de las distribuciones de tamaño de partícula obtenidas con el modelo fractal como insumo en ecuaciones de transferencia edafológica.

Investigar sobre la distribución y calidad de la materia orgánica en las fracciones granulométricas correspondientes al pretratamiento con materia orgánica y elaborar un índice de calidad físico químico empleando la textura aparente.

Ampliar el tamaño muestral sobre el comportamiento de las fracciones aparentes en función del carbono orgánico asociado a la fracción mineral en suelos más gruesos que franco.

Estudiar la aplicación de los parámetros que arroja el modelo con propiedades del suelo, por ejemplo, en el movimiento de fluidos

Estudiar la aplicación de los parámetros fractales al estudio de los procesos de formación del suelo, en la tesis como ejemplo se observó la variación con el proceso de argiluviación

\subsubsection{Como aplicaciones, futuras se podría mencionar}

Ampliar el alcance del modelo fractal en el estudio de las distribuciones de tamaños de partículas a otras áreas geográficas.

Incorporar el modelo fractal a las bases de datos digitales de los suelos.

Elaborar mapas con valores de Iso C e Iso D. 
Bibliografía 


\section{Bibliografía}

Amato M y Ladd JN. 1992. Decomposition of 14C-labelled glucose and legume material in soils: Properties influencing the accumulation of organic residue $\mathrm{C}$ and microbial biomass $\mathrm{C}$. Soil Biol. Biochem. 24:455-464.

Arya LM y Paris JF, 1981. A physico-empirical model to predict the soil misture characteristic curve from particle-size distribution and bulk density data. Soil Sci. Soc. Am. J. 45, 1023-1030.

Avnir D, Biham O, Lidar D y Malcai O. 1998. Is the Geometry of Nature Fractal? Science, vol. 279 no. 5347 pp. 39-40.

Barak B, Seybold CA y McSweeney K. 1996. Self similitude and fractal dimension of sand grains. Soil Sci. Soc. Am. J. 60: 72-76.

Bartoli F, Philippy R, Doirisse M, Niquet S y Dubuit M. 1991. Structure and self-similarity in silty and sandy soils: The fractal approach. J. Soil Sci. 42: 167-185.

Beuselinck L, Govers G, Poesen J, Degraer G y Froyen L. 1998. Grain size analysis by laser diffractometry: comparison with the sieve pipette method. Catena, 32 193-208.

Behzad G y Daigle H. 2015. Fractal dimension of soil fragment mass-size distribution: A critical analysis. Geoderma 245-246 98-103.

Bittelli M, Campbell GS y Flury M. 1999. Characterization of particle-size distribution in soil with a fragmentation model. Soil Sci. Soc. Am. J. 63, 782-788.

Botula YD, Cornelis WM, Baert G, Mafuka P y Van Ranst E. 2013 Particle size distribution models for soils of the humid tropics J Soils Sediments 13:686-698.

Bronick CJ y Lal R. 2005. Soil structure and management: a review. Geoderma 124: 322.

Campbell G y Shiozawa S. 1992. Indirect methods for estimating the hidraulic properties of unsaturated soils. University of California, Riverside., Ch. Prediction of hydraulic properties of soils using particle-size distribution and bulk density data, pp. 317-328.

Cappannini D y Dominguez O. 1961. Los principales ambientes geoedafológicos de la Prov. de Buenos Aires. IDIA, 63: 33-39.

Caruso T, Barto EK, Siddiky MRK, Smigelski J y Rillig MC. 2011. Are power laws that estimate fractal dimension a good descriptor of soil structure and its link to soil biological properties? Soil Biology \& Biochemistry 43, 359-366. 
Day PR. 1965. Particle fractionation and particle-size analysis. In: Black, CA, et al. (Ed.), Methods of Soil Analysis, Part 1, pp. 545-567.

Di Rienzo JA; F Casanoves; MG Balzarini; L Gonzalez; M Tablada y CW Robledo, InfoStat versión 2018, Grupo InfoStat, FCA, Universidad Nacional de Córdoba, Argentina, URL http://www.infostat.com.ar

Dur JC, Elsass F, Chaplain V y Tessier D. 2004. The relationship between particle size distribution by laser granulometry and image analysis by transmission electron microscopy in a soil clay fraction. Eur. J. S. Sci. 55, 265-270.

Eshel G, Levy GJ, Mingelgrin U y Singer MJ. 2004. Critical evaluation of the use of the laser diffraction for particle size distribution analysis. Soil Sci. Soc. Am. J. 68, 736-743.

Filgueira RR, Pachepsky Ya y Fournier LL. 2003. Time-mass scaling in soil texture analysis. Soil Sci. Soc. Am. J. 67: 1703-1706. ISSN 0361-5995.

Filgueira RR, Gelati PR, Fournier LL y Sarli GO. 2004. ¿Es la distribución de tamaño de partículas de un suelo siempre fractal? Actas XIX Congreso Argentino de la Ciencia del Suelo. Paraná Entre Ríos, Argentina.

Filgueira RR, Fournier LL, Sarli GO y Soracco CG. 2005. Distribución de partículas y agregados de suelos: el modelo fractal. En: Reología del suelo agrícola bajo tráfico. Ed. D Jorajuría Editorial de la Universidad de La Plata. EDULP. Pp. 21-38. ISBN 950-34-0334-0.

Filgueira RR, Fournier LL, Cerisola Cl, Gelati PR y García MG. 2006 (a). Particle-size distribution in soils; a critical study of the fractal model validation. Geoderma, Volume 134, Issues 3-4, 327-334.

Filgueira RR, Gelati PR, Soracco CG y Sarli GO. 2006 (b). Textura: propuesta alternativa para la determinación del porcentaje de arcilla por medio del hidrómetro en "XX Congreso Argentino de la Ciencia del Suelo y I Reunión de Suelos de la Región Andina ". Salta.

Filgueira RR, Gelati PR, Fournier LL, Sarli GO y Soracco CG. 2009. Distribución de tamaño de partículas del suelo; su estudio con un modelo fractal. XVIII Congreso Latinoamericano de la Ciencia del Suelo. Costa Rica.

Gee GW y Bauder JW. 1986. Particle size analysis. methods of soil analysis, part 1. Physical and minerological methods. Agronomy Monograph № 9. Second Edition. Pp. 383-411.

Gee GW y Orr D. 2002 Particle-size analysis. JH Dame, G Topp (Eds.), Methods of Soil Analysis, Part 4 - Physical Methods, Soil Science Society of America, Madison, WI, pp. 255-293. 
Gelati PR, Sarli GO, Lozano LA y Filgueira RR. 2008. Arcilla: tiempos necesarios para estimar su contenido con hidrómetro según pretratamientos. XXI Congreso Argentino de la Ciencia del Suelo. Potrero de los Funes, San Luis. Argentina.

Gelati PR; Sarli GO; Soracco CG; Lozano LA y Filgueira RR. 2009. Influencia de la materia orgánica en la superficie específica de un Argiudol típico. Revista de la Facultad de Ciencias Agrarias, Universidad Nacional de Cuyo. Tomo XLI. N². ISSN 0370-4661

Gelati PR, Sarli GO, Lozano LA, Soracco CG y Filgueira RR. 2010. Microagregación: análisis mediante curvas de sedimentación, XXII Congreso Argentino de la Ciencia del Suelo. Rosario, Santa Fé, Argentina.

Gelati PR, Sarli GO y Filgueira RR. 2016. Estudio fractal de la distribución de tamaño de partículas y pseudopartículas en suelos de la pradera pampeana. Actas del XXV Congreso Argentino de la Ciencia del Suelo. Río Cuarto, Córdoba, Argentina.

Gelati PR, Sarli GO y Filgueira, RR. 2018. Modelo fractal aplicado a la distribución de tamaño de partículas en suelos francos y franco arenosos, XXVI Congreso Argentino de la Ciencia del Suelo. San Miguel de Tucumán, Tucumán, Argentina.

Gelati PR, Sarli GO, Lozano LA y Filgueira RR. 2019. Estudio fractal de partículas y pseudopartículas en suelos de la pradera pampeana". Revista de AACS. 37 (1): 147-157.

Gonzalez Bonorino F.1966. J. of Sed. Petrology, 36(4):1026-1035.

Gunal H, Ersahin S, Buket Y, Budak M y Acir N. 2011. Soil Particle Size Distribution and Solid Fractal Dimension as Influenced by Pretreatments. Tarim Bilimleri Dergisi. J Agr Sci. 17. 217-229.

Hassink J. 1994. Effects of soil texture and grassland management on soil organic $C$ and $\mathrm{N}$ and rates of $\mathrm{C}$ and $\mathrm{N}$ mineralization. Soil Biol. Biochem. 26: 1221-1231.

Hyslip J y Vallejo L. 1997. Fractal analysis of the roughness and size distribution of granular materials. Engineering Geology 48, 231-244.

Iriondo M.1990. Quaternary of South America and Antartic Peninsula. 6: 297-308.

Iriondo M.1992. Geomorphological Map of South American Plains. Escala 1:5.000.000

Iriondo M y Kröhling D.1995. Comunicaciones. Museo Prov. F.Ameghino, 5(1), 68 p. 
Iriondo M y Kröhling D.1996. Actas XIII Congreso Geológico Argentino. Tomo IV: 27-48.

Jastrow JT, Boutton y Miller R. 1996. Carbon dynamics of aggregate-associated organic matter estimated by carbon-13 natural abundance. Soil Sci. Soc. Am. J. 60: 801-807.

Jenkinson DS. 1988. Soil organic matter and its dynamics. In: Wild, A. (Ed.). Russels soil conditions and plant growth. 11th ed. Longman. New York, USA. p. 564-607.

Knösche C, Friedrich H y Stintz M. 1997. Determination of particle size distribution and electrokinetic propierties with the acustosizer in comparison with other methods. Particle and Particle Systems Characterization, 14, 175-180.

Konert M y Vandenberghe J. 1997. Comparison of laser grain size analysis with pipette and sieve analysis: a solution for the underestimation of the clay fraction. Sedimentology 44, 523 -535 .

Korvin G. 1992. Fractal Methods in the Earth Sciences. Elsevier, Amsterdam. 191 p.

Le Bissonnais Y. 1996. Aggregate stability and assessment of soil crustabilityand erodibility: I. Theory and methodology. Eur. J. Soil Sci., 47,425-431.

Mandelbrot B. 1982. The fractal geometry of nature. WH Freeman and Company. New York.

Matus FJ y Maire GChR. 2000. Relación entre la materia orgánica del suelo, textura del suelo y tasas de mineralización de carbono y nitrógeno. Agric. Téc.[online]. 2000, vol.60, n.2, pp.112-126.

Millán H, Gonzalez-Posada M, Aguilar M, Domínguez J y Céspedes L. 2003. On the fractal scaling of soil data. Particle size distributions. Geoderma 117, 117-128.

McManus J. 1988. Grain size determination and interpretation. En Techniques in Sedimentology, Tucker M. (ed.). Blackwell: Oxford;63 - 85.

Morrás H. 2003. Distribución y origen de sedimentos loéssicos superficiales de la Pampa norte en base a la mineralogía de arenas. Resultados preliminares. AAS Revista, vol. 10 Nº1:5364 Asociación Argentina de Sedimentología. ISSN 03281159.

Morrás H y Cruzate GA. 2000. Clasificación textural y distribución espacial del material originario de los suelos de la Pampa Norte. XVII Congreso Argentino de la Ciencia del Suelo. AACS en el 2000. Mar del Plata, Pcia. de Buenos Aires.

Oades J y Waters A. 1991. Aggregate hierarchy in soil. Austr. J. Soil Res. 29: 815-828. 
Pachepsky Ya y Rawls JM. 2004. Development of Pedotransfer Functions in Soil Hydrology. Development in Soil Sciences (Vol 30). Amsterdam. The Netherlands. 504 p.

Perfect E, Díaz Zorita M y Grove J. 2002. A prefractal model for predicting soil fragment size distributions. Soil Till Res. 36,1-20.

Porta Casanellas J, Lopez-Acevedo Reguerín y Roquero De Laburu C. 1999. Edafología para la agricultura y el medio ambiente. Ediciones Mundi-Prensa.

Prosperini N y Perugini D. 2008. Particle size distributions of some soils from the Umbria Region (Italy): fractal analysis and numerical modelling. Geoderma 145, 185 -195.

SAMLA. 2004. Secretaría de Agricultura, Ganadería, Pesca y Alimentación de la Nación Argentina. Dirección de Producción Agrícola. Sistema de Apoyo Metodológico a los Laboratorios de Análisis de Suelos Agua, Vegetales y Enmiendas Orgánicas.

Sander L M. 1986. Fractal growth processes. Nature 322: 789-793.

Sandoval-Estrada M, Stolpe N, Zagal-Venegas E, Mardones-Flores M y Celis-Hidalgo J. 2008. Aporte de carbono orgánico de la labranza cero y su impacto en la estructura de un Andisol de la precordillera andina chilena. Agrociencia 42: 139-149.

Six J, Paustian K, Elliott E y Combrink C. 2000. Soil structure and organic matter: I. Distribution of aggregate-size classes and aggregateassociated carbon. Soil Sci. Soc. Am. J. 64: 681-689.

Six J, Bossuyt H, Degryze S y Denef K. 2004. A history of research on the link between (micro) aggregates, soil biota and soil organic matter dinamic. Soil Till Res. 79, 7-31.

Stanchi S, Bonifacio E, Zanini E y Pachepsky Ya. 2006. Presence and prediction of fractal behaviour in particle-size distributions as affected by the sample pretreatment and soil properties. Soil Sci. 171 (4), 283-292.

Stanchi S, Bonifacio E y Zanini E. 2008. Mass-size fractal dimension of primary and aggregate particles and soil profile development. Soil Sci. 173 (2), 87-95.

Taguas F, Martín M y Perfect E. 1999. Simulation and testing of self-similar estructures for soil particle size distributions using iterated function systems. Geoderma 88, 191 -203.

Tisdall J y Oades. 1982. Organic matter and water-stable aggregates in soils. J. Soil Sci. 33: $141-163$. 
Turcotte DL. 1986. Fractals and fragmentation. J. Geophys. Res. 91, 1921-1926.

Tyler SW y Wheatcraft SW. 1989. Application of fractal mathematics to soils water retention estimation. Soil Sci. Soc. Am. J. 5, 987 - 996.

Tyler SW y Wheatcraft SW. 1992. Fractal scaling of soil particle-size distributions: analysis and limitations. Soil Sci. Soc. Am. J. 56, 362-369.

Valdez Cepeda RD y Olivares-Sáenz E. 1998. Geometría fractal en la ciencia del suelo. Terra, 16(3) 277-288.

Vdovic N, Obhodas J y Pikelj K. 2010.Resvisiting the particle-size distribution of soils: comparison of different methods and simple pre-treatments. Eur J Soil Sci, 61, 854-864.

Vivas Miranda JG, García Chomiczak S y Paz González A. 1999. Teoría fractal y efecto de cambio de escala: aplicación al estudio de la porosidad del suelo. Cuadernos do Laboratorio Xeolóxico de Laxe, 1999, 24: 99-119 ISSN: 00213-4497

Wu Q, Borkovec M y Sticher S. 1993. On particle size distributions in soils. Soil Sci. Soc. Am. J. 57, 883-890.

Young IM y Crawford JW. 1991. The fractal structure of soil aggregates: Its measurement and interpretation. J. Soil Sci. 42: 187-192. 
Anexo 


\section{$9 \quad$ Anexo}

Se agregan fotos del laboratorio, mientras se realizaron las determinaciones como también algunas Figuras correspondientes a resultados empleados en la tesis. 
Fotos de laboratorio

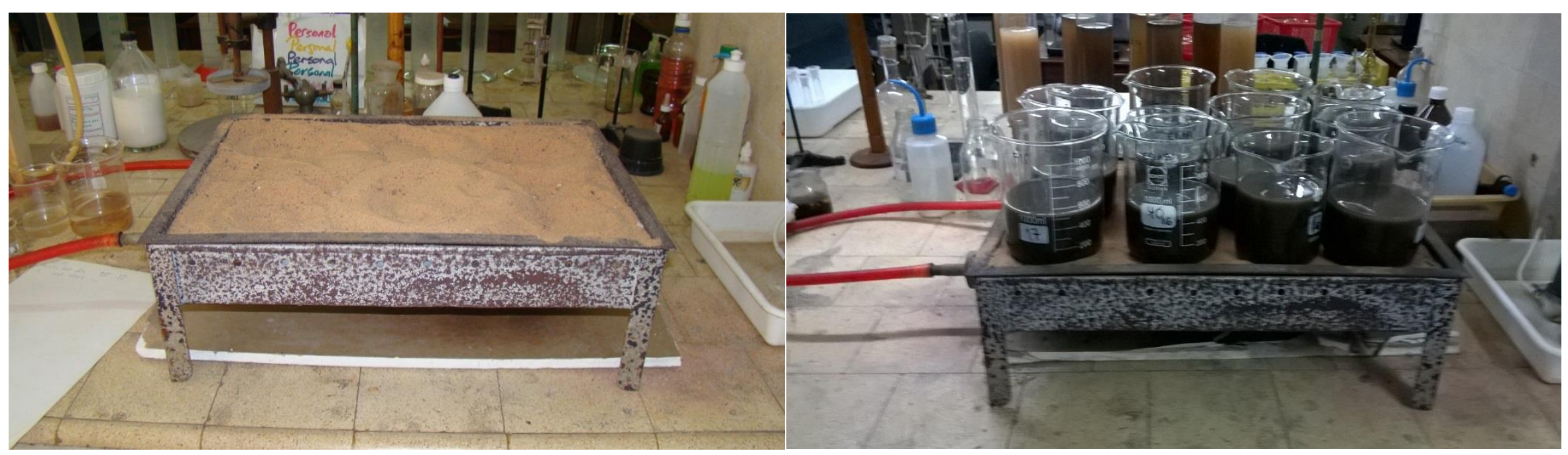

Imagen 9-1 Cama de arena caliente, aumenta la temperatura y acelera el proceso de eliminar materia orgánica de las muestras de suelo empelando peróxido de hidrógeno 


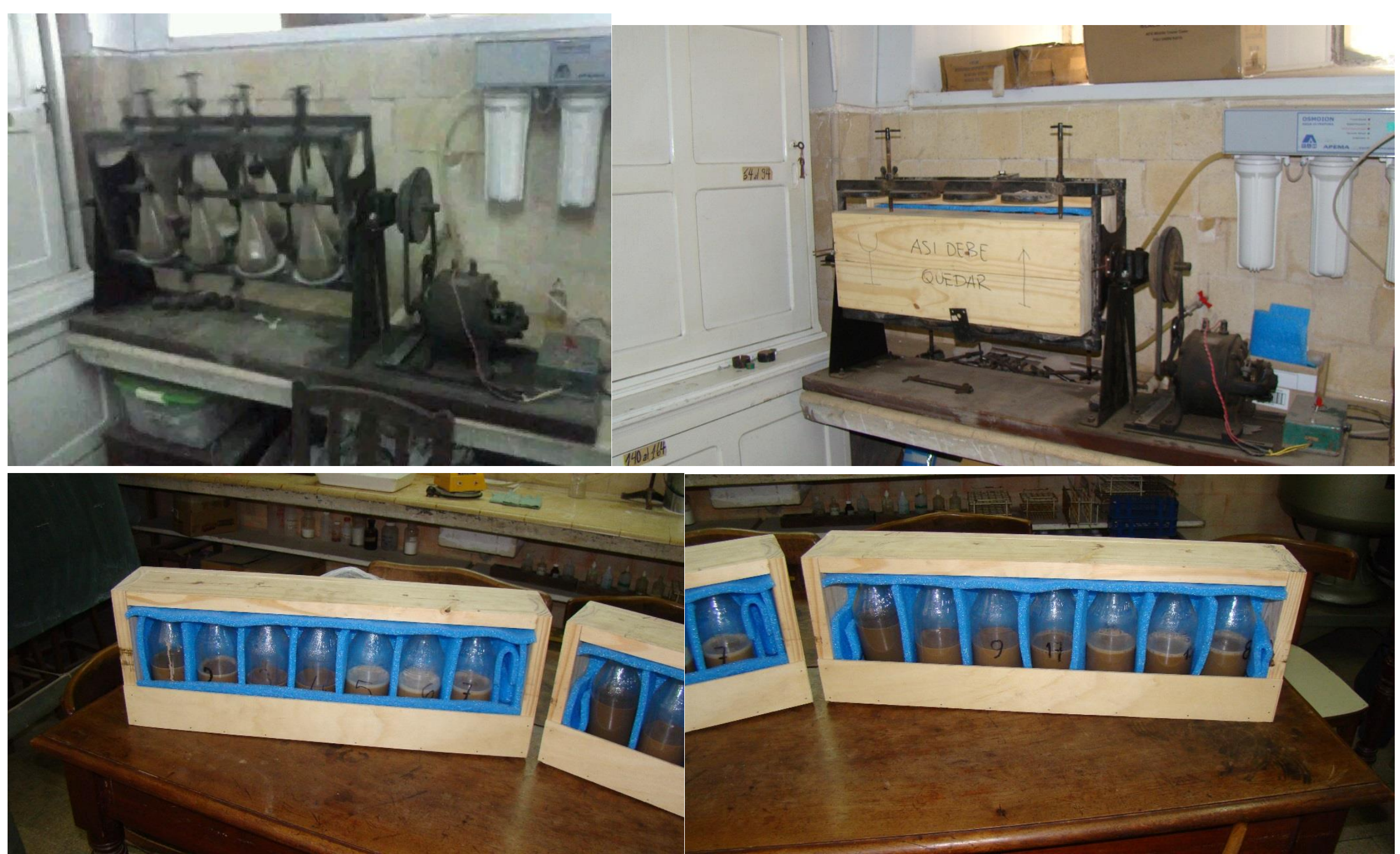

Imagen 9-2 Agitador rotativo, para dispersar mecánicamente, las muestras de suelos sin materia orgánica con dispersante químico y esferitas de vidrio para intensificar el proceso 

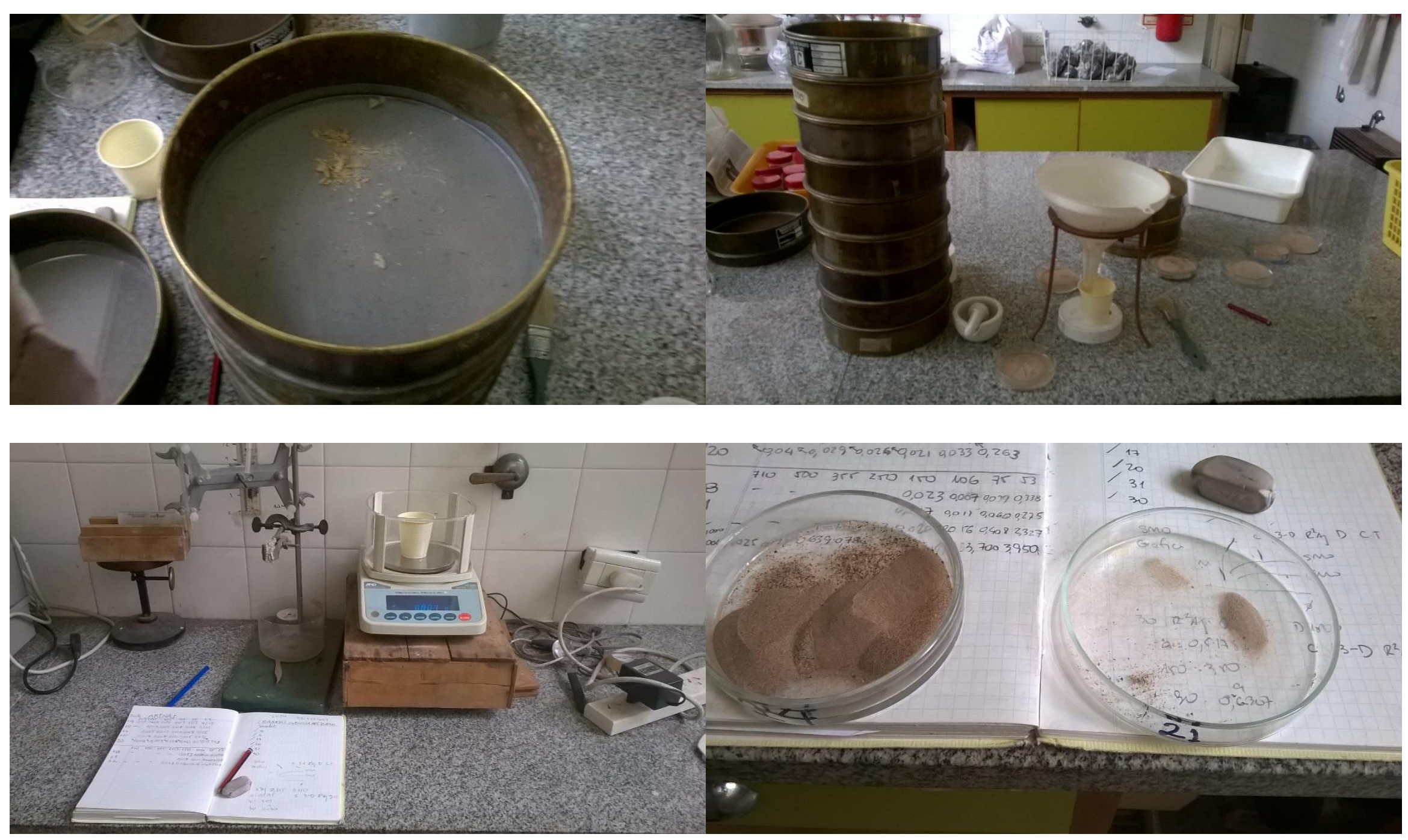

Imagen 9-3. Tamizado y cuantificación de las arenas en las muestras sin materia orgánica, torre de tamices, pesado y registro 

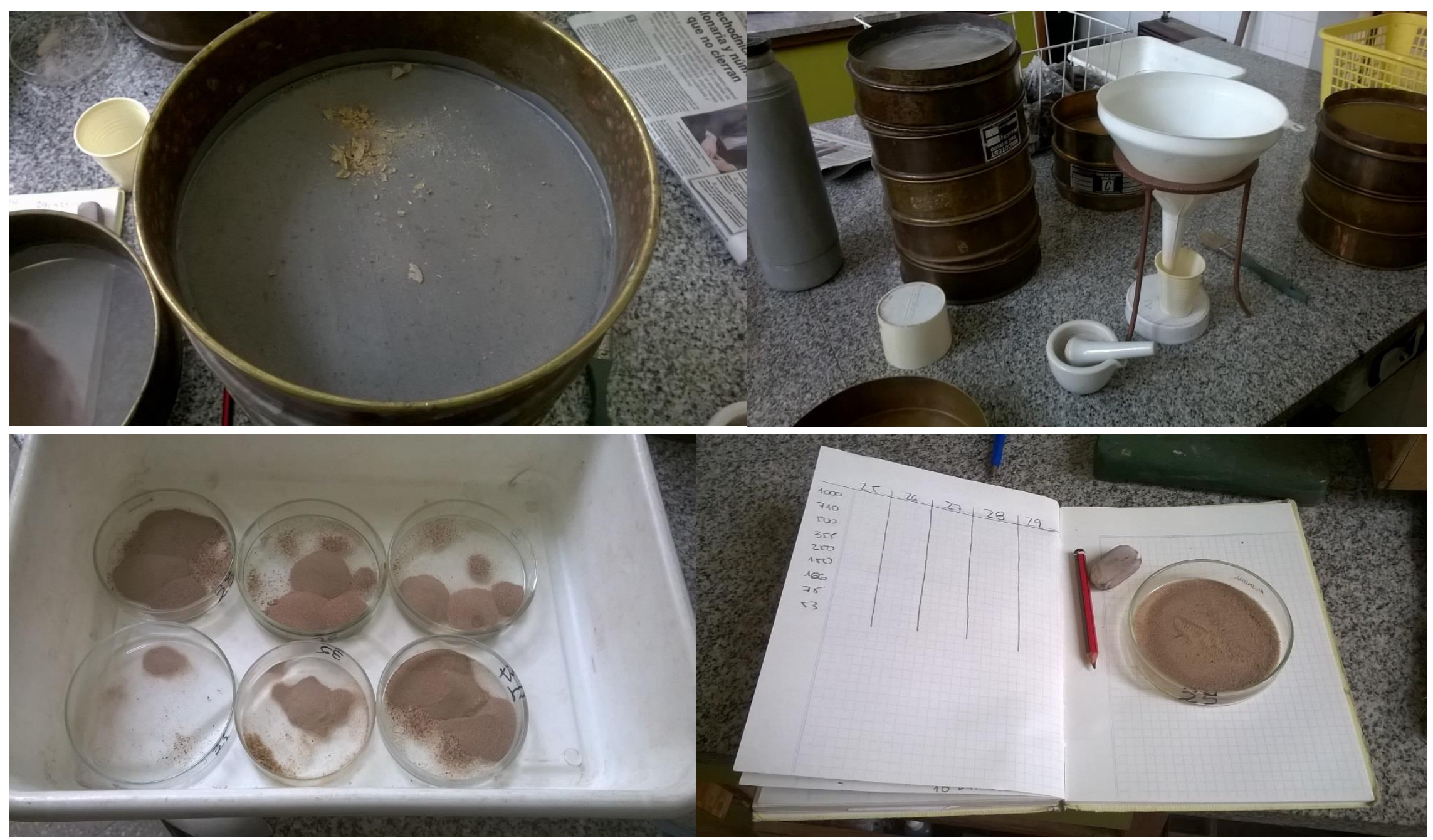

Imagen 9-4.Otras imágenes del proceso de cuantificación de las arenas. 


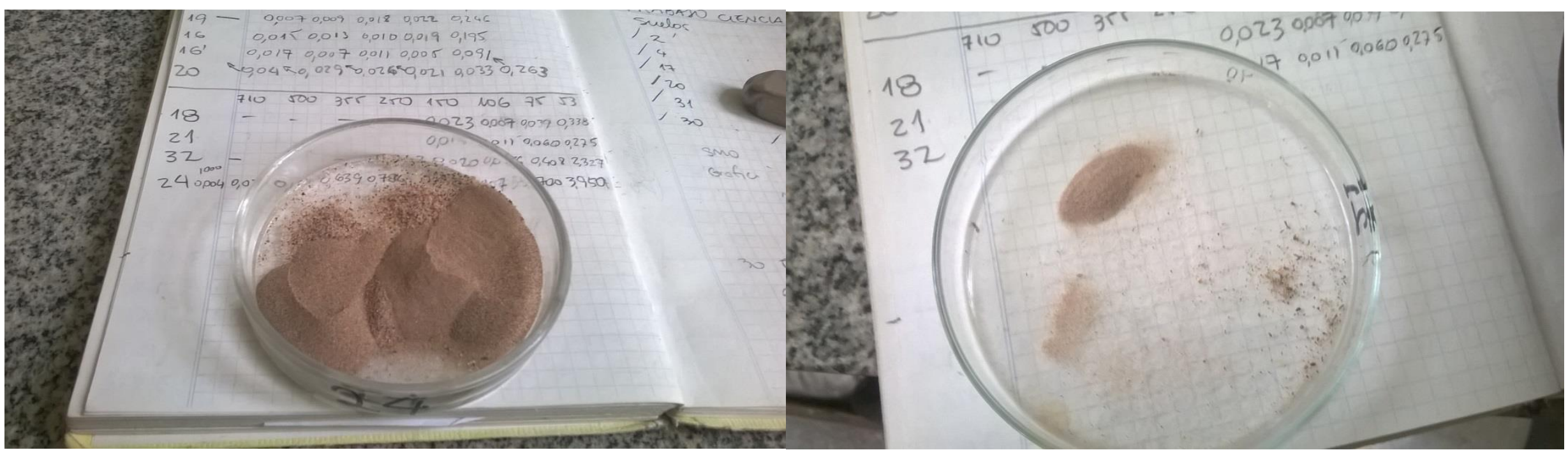

Imagen 9-5 Contraste de la cantidad de arenas de un suelo del grupo 1 (izquierda) y otro del grupo 3 (derecha) 

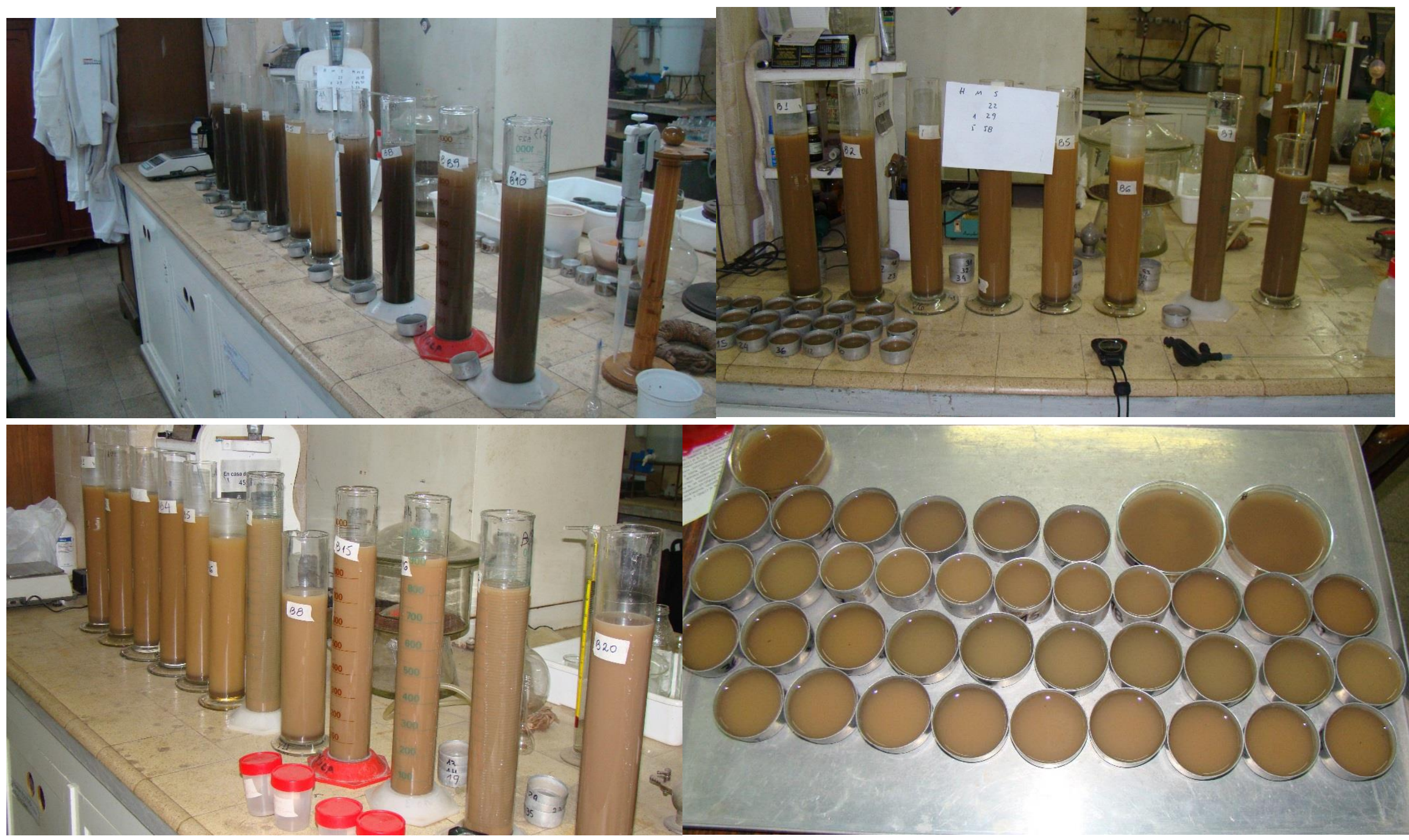

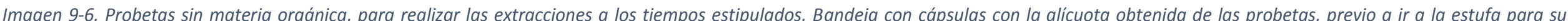
evaporación. Se aprecia el color claro de las suspensiones 


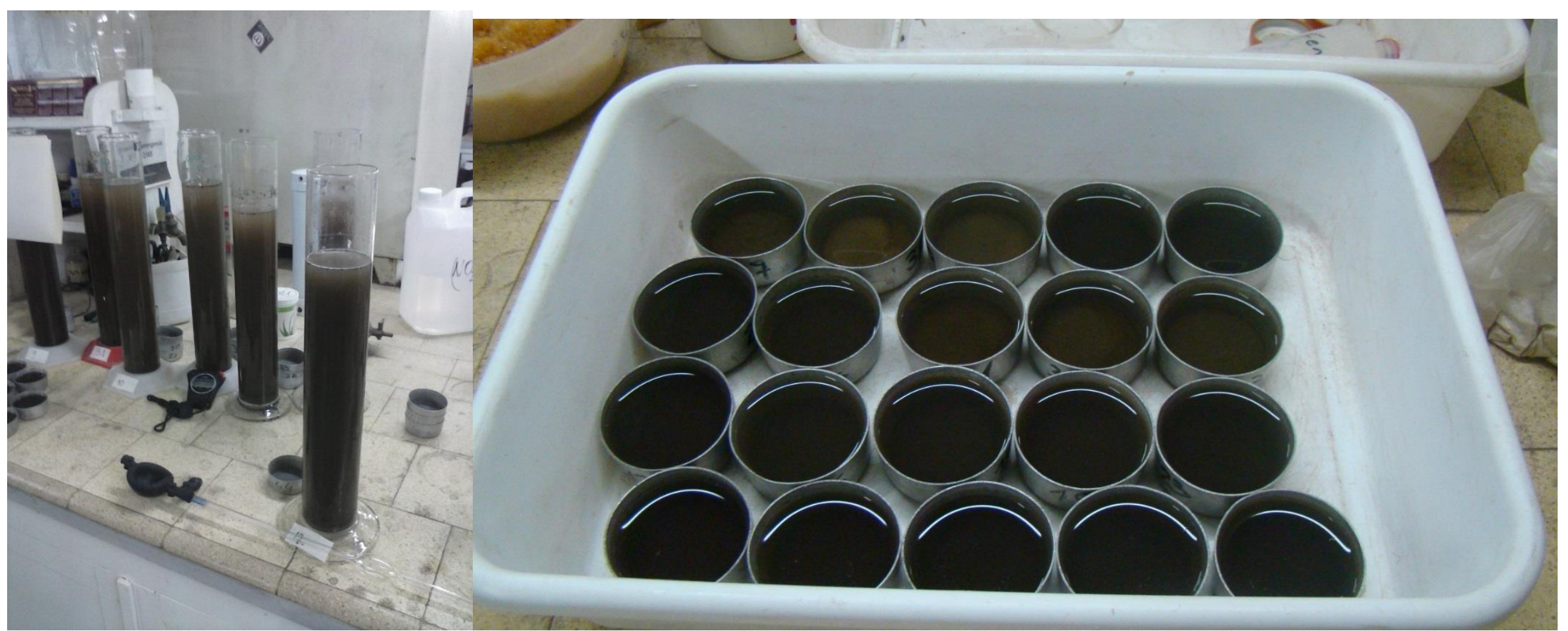

Imagen 9-7. Probetas con materia orgánica, para evaluar la textura aparente, se aprecia el color de oscuro de las muestras en las probetas y las cápsulas 

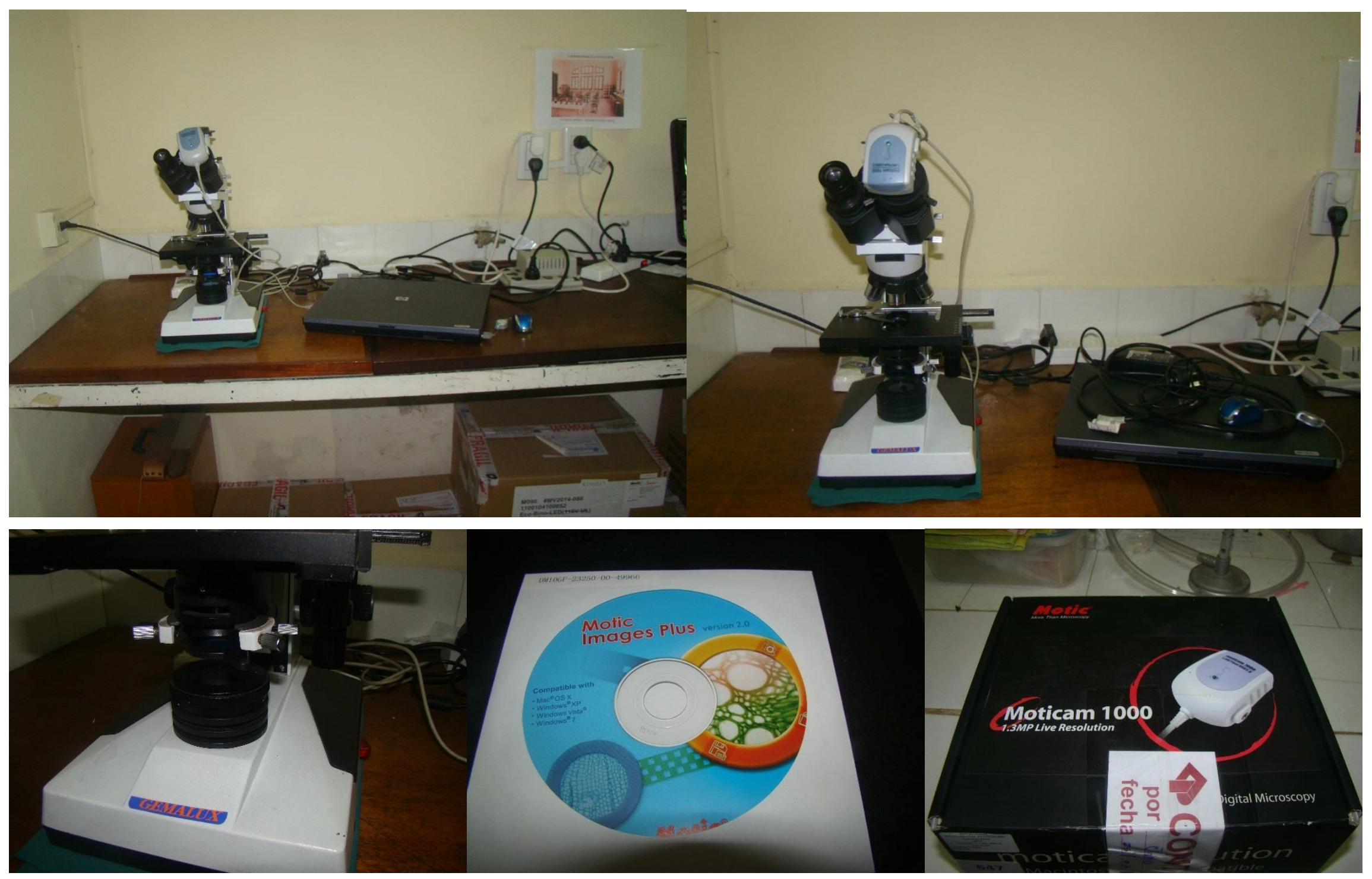

Imagen 9-8. Microscopio óptico con cámara y software para tomar las fotos obtenida en la tesis 
Los siguientes vínculos, abren hojas Excel, con gráficos de las sedimentaciones SMO y CMO.

VinculosIG1 graficos SMO CMO.xlsx

VinculosIG2 graficos SMO CMO.xlsx

VinculosIG3 graficos SMO CMO.xlsx 


\section{Mineralogía de los suelos}

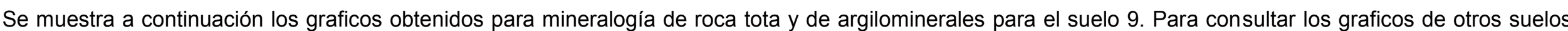
analizados pueden observarse en $\underline{\text { Vinculos\Mineralogía }}$ 


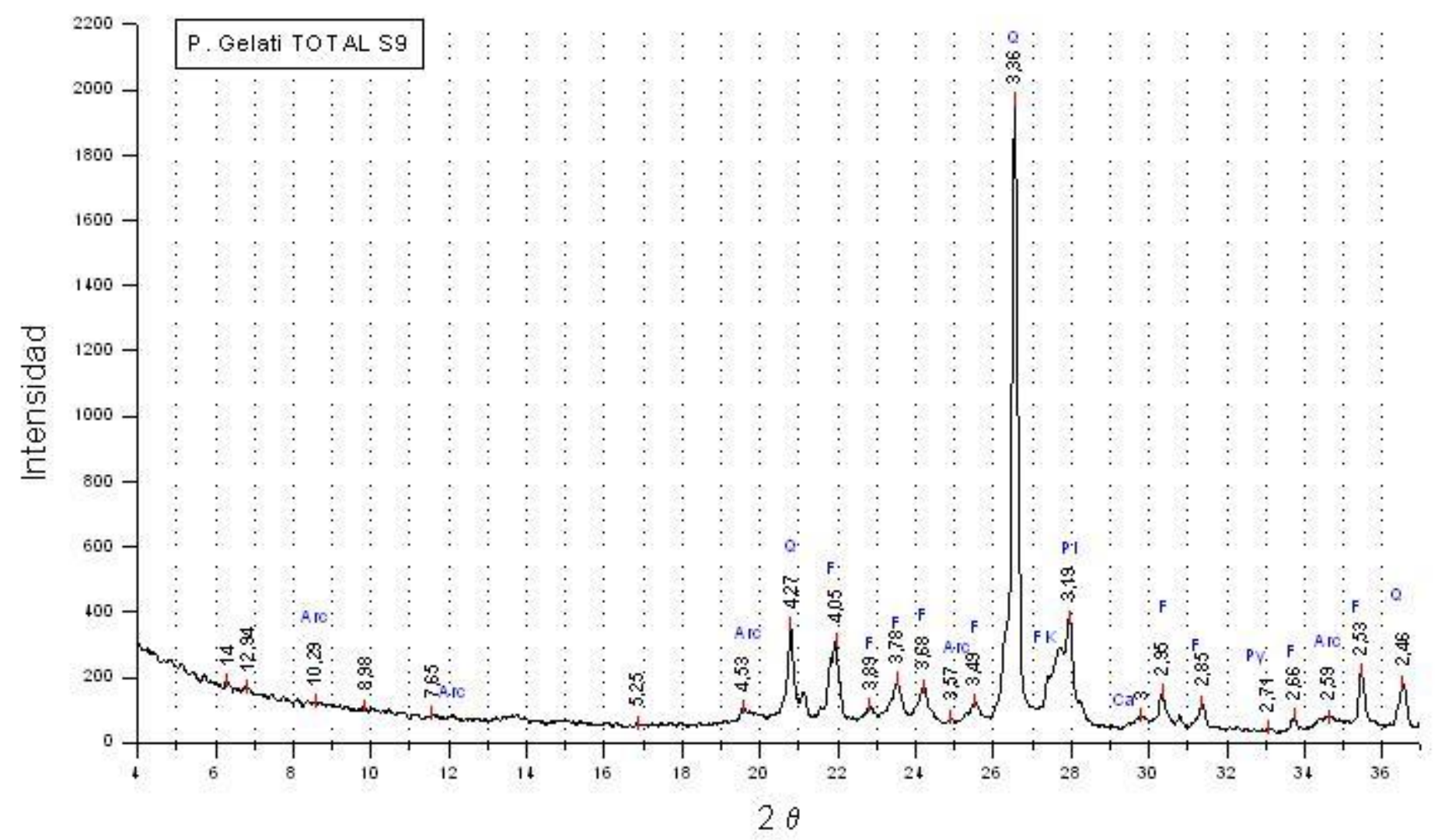




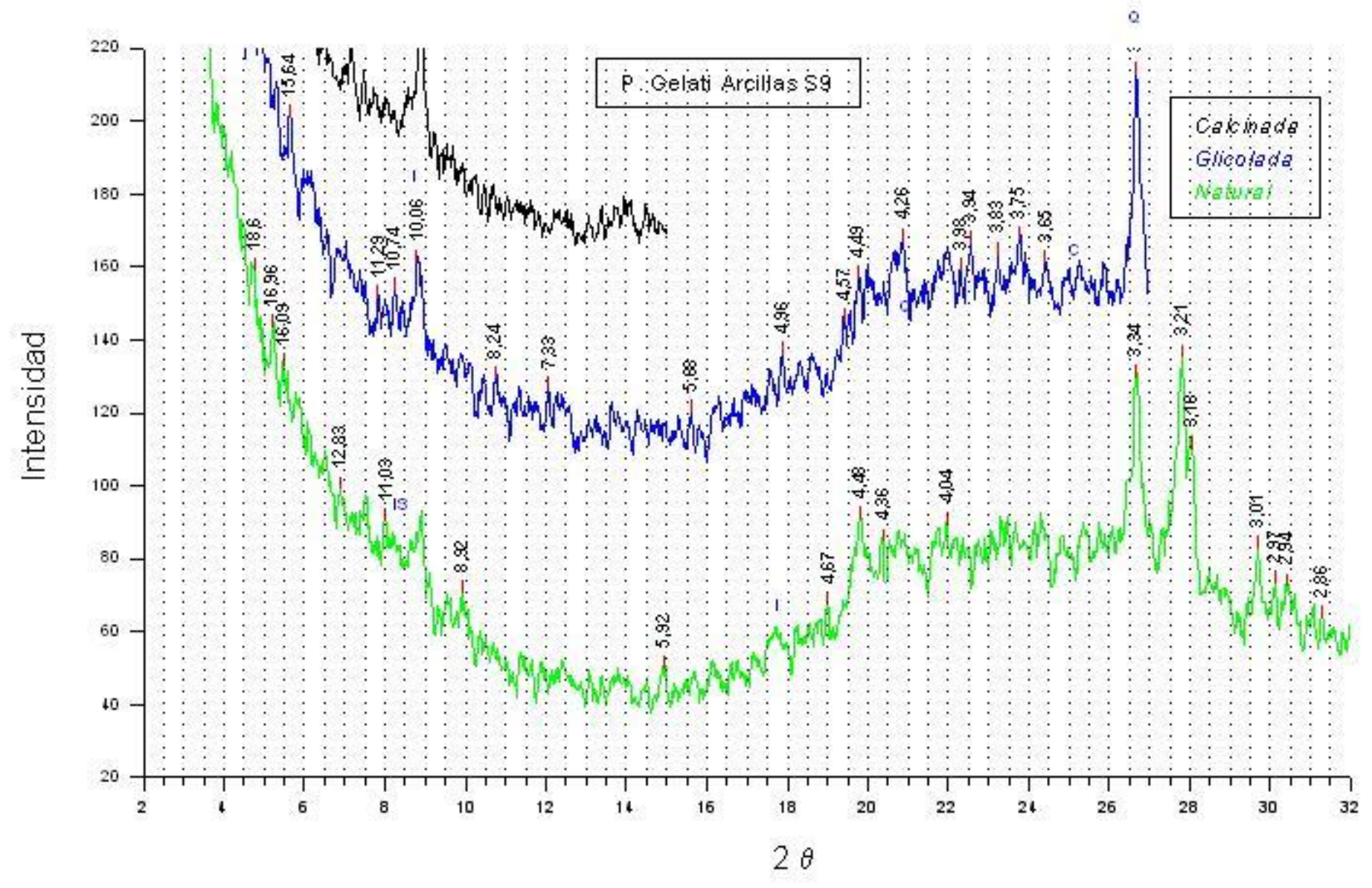

